\title{
THE SECOND FERMI LARGE AREA TELESCOPE CATALOG OF GAMMA-RAY PULSARS
}

A. A. Abdo ${ }^{1,88}$, M. Ajello ${ }^{2}$, A. Allafort ${ }^{3}$, L. Baldini ${ }^{4}$, J. Ballet ${ }^{5}$, G. Barbiellini ${ }^{6,7}$, M. G. Baring ${ }^{8}$, D. Bastieri ${ }^{9}, 10$, A. Belfiore ${ }^{11,12,13}$, R. Bellazzini ${ }^{14}$, B. Bhattacharyya ${ }^{15}$, E. Bissaldi ${ }^{16}$, E. D. Bloom ${ }^{3}$, E. Bonamente ${ }^{17,18}$, E. BottaCini ${ }^{3}$, T. J. Brandt ${ }^{19}$, J. Bregeon ${ }^{14}$, M. Brigida ${ }^{20,21}$, P. Bruel ${ }^{22}$, R. Buehler ${ }^{23}$, M. Burgay ${ }^{24}$, T. H. Burnett ${ }^{25}$, G. Busetto ${ }^{9,10}$, S. Buson ${ }^{9}, 10$, G. A. Caliandro ${ }^{26}$, R. A. Cameron ${ }^{3}$, F. Camilo ${ }^{27,28}$, P. A. Caraveo ${ }^{13}$, J. M. Casandjian ${ }^{5}$, C. CeCChi ${ }^{17,18}$, Ö. Çelik ${ }^{19,29,30}$, E. Charles ${ }^{3}$, S. Chaty ${ }^{5}$, R. C. G. Chaves ${ }^{5}$, A. CheKhtman ${ }^{1,88}$, A. W. Chen ${ }^{13}$, J. Chiang ${ }^{3}$, G. Chiaro ${ }^{10}$, S. Ciprini ${ }^{31,32}$, R. Clauds ${ }^{3}$, I. Cognard ${ }^{33}$, J. Cohen-TANugi ${ }^{34}$, L. R. CominskY ${ }^{35}$, J. ConRad $^{36,37,38,89}$, S. Cutini ${ }^{31,32}$, F. D’Ammando ${ }^{39}$, A. De Angelis ${ }^{40}$, M. E. DeCesar ${ }^{19,41}$, A. De LuCA ${ }^{42}$, P. R. Den Hartog ${ }^{3}$, F. De Palma ${ }^{20,21}$, C. D. Dermer ${ }^{43}$, G. Desvignes ${ }^{33,44}$, S. W. Digel ${ }^{3}$, L. Di Venere ${ }^{3}$, P. S. Drell ${ }^{3}$, A. Drlica-Wagner ${ }^{3}$, R. Dubois ${ }^{3}$, D. Dumora ${ }^{45}$,

C. M. Espinoza ${ }^{46}$, L. Falletti ${ }^{34}$, C. Favuzzi $^{20,21}$, E. C. Ferrara $^{19}$, W. B. Focke ${ }^{3}$, A. Franckowiak ${ }^{3}$, P. C. C. Freire ${ }^{44}$,

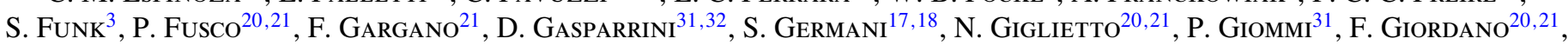

M. Girolettis ${ }^{39}$, T. Glanzman ${ }^{3}$, G. Godfrey ${ }^{3}$, E. V. Gotthelr ${ }^{27}$, I. A. Grenier ${ }^{5}$, M.-H. Grondin ${ }^{47,48}$, J. E. Grove ${ }^{43}$, L. Guillemot ${ }^{44}$, S. Guiriec ${ }^{19,90}$, D. Hadasch ${ }^{26}$, Y. Hanabata ${ }^{49}$, A. K. Harding ${ }^{19}$, M. Hayashida ${ }^{3,50}$, E. Hays ${ }^{19}$,

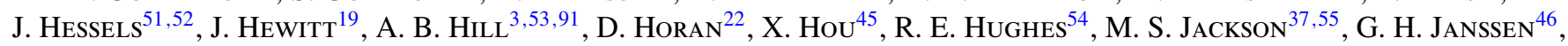

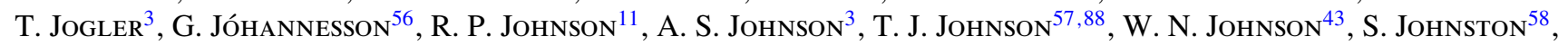

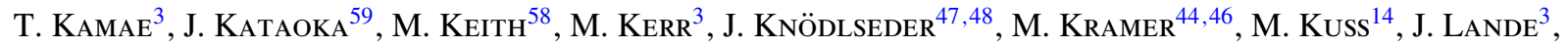

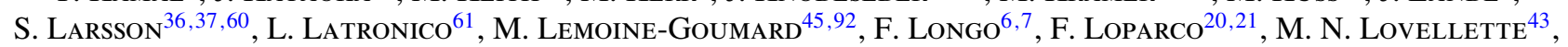

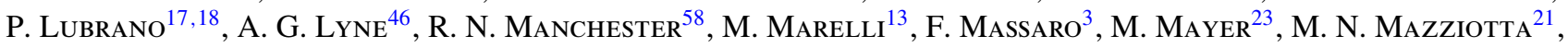
J. E. McEnery ${ }^{19,41}$, M. A. McLaughlin ${ }^{62}$, J. Mehauli ${ }^{45}$, P. F. Michelson ${ }^{3}$, R. P. Mignani ${ }^{13,63,64}$, W. Mitthumsiri ${ }^{3}$, T. Mizuno ${ }^{65}$, A. A. Moiseev ${ }^{29,41}$, M. E. Monzani ${ }^{3}$, A. Morselli ${ }^{66}$, I. V. MosKalenko ${ }^{3}$, S. Murgia ${ }^{3}$, T. NaKamori ${ }^{67}$, R. Nemmen ${ }^{19}$, E. Nuss ${ }^{34}$, M. Ohno ${ }^{49}$, T. Ohsugi ${ }^{65}$, M. Orienti ${ }^{39}$, E. Orlando ${ }^{3}$, J. F. Ormes ${ }^{68}$, D. Paneque ${ }^{3,69}$, J. H. Panetta ${ }^{3}$, D. Parent ${ }^{1,88}$, J. S. Perkins ${ }^{19}$, M. Pesce-Rollins ${ }^{14}$, M. Pierbattista ${ }^{13}$, F. Piron ${ }^{34}$, G. Pivato ${ }^{10}$, H. J. Pletsch ${ }^{70,71}$,

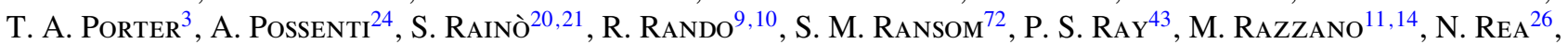
A. Reimer ${ }^{3,73}$, O. Reimer ${ }^{3,73}$, N. Renault ${ }^{5}$, T. Reposeur ${ }^{45}$, S. Ritz ${ }^{11}$, R. W. RomAni ${ }^{3}$, M. Roth ${ }^{25}$, R. Rousseau ${ }^{45}$, J. Roy $^{15}$, J. Ruan ${ }^{74}$, A. Sartori ${ }^{13}$, P. M. Saz Parkinson ${ }^{11}$, J. D. Scargle ${ }^{75}$, A. Schulz ${ }^{23}$, C. Sgrò ${ }^{14}$, R. Shannon ${ }^{58}$, E. J. Siskind ${ }^{76}$,

D. A. Smith ${ }^{45}$, G. Spandre ${ }^{14}$, P. Spinelli ${ }^{20,21}$, B. W. Stappers ${ }^{46}$, A. W. Strong ${ }^{77}$, D. J. Suson ${ }^{78}$, H. Takahashi ${ }^{49}$,

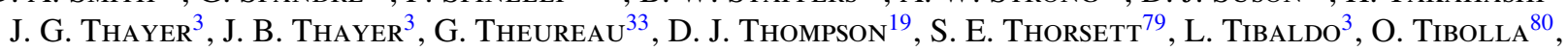

M. Tinivella ${ }^{14}$, D. F. Torres ${ }^{26,81}$, G. Tosti ${ }^{17,18}$, E. Troja ${ }^{19,41}$, Y. Uchiyama ${ }^{82}$, T. L. Usher ${ }^{3}$, J. VAndenbroucke $^{3}$,

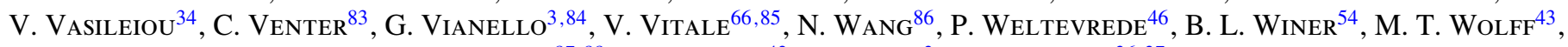
D. L. Wood ${ }^{87,88}$, K. S. WoOd ${ }^{43}$, M. WoOd ${ }^{3}$, AND Z. YANG ${ }^{36,37}$

${ }^{1}$ Center for Earth Observing and Space Research, College of Science, George Mason University, Fairfax, VA 22030, USA

${ }^{2}$ Space Sciences Laboratory, 7 Gauss Way, University of California, Berkeley, CA 94720-7450, USA

${ }^{3}$ W. W. Hansen Experimental Physics Laboratory, Kavli Institute for Particle Astrophysics and Cosmology, Department of Physics

and SLAC National Accelerator Laboratory, Stanford University, Stanford, CA 94305, USA; hartog@ stanford.edu, kerrm@ stanford.edu, joshualande@ gmail.com

${ }^{4}$ Università di Pisa and Istituto Nazionale di Fisica Nucleare, Sezione di Pisa, I-56127 Pisa, Italy

${ }^{5}$ Laboratoire AIM, CEA-IRFU/CNRS/Université Paris Diderot, Service d'Astrophysique, CEA Saclay, F-91191 Gif sur Yvette, France

${ }^{6}$ Istituto Nazionale di Fisica Nucleare, Sezione di Trieste, I-34127 Trieste, Italy

${ }^{7}$ Dipartimento di Fisica, Università di Trieste, I-34127 Trieste, Italy

${ }^{8}$ Rice University, Department of Physics and Astronomy, MS-108, P.O. Box 1892, Houston, TX 77251, USA

${ }_{9}$ Istituto Nazionale di Fisica Nucleare, Sezione di Padova, I-35131 Padova, Italy

${ }^{10}$ Dipartimento di Fisica e Astronomia “G. Galilei,” Università di Padova, I-35131 Padova, Italy

${ }^{11}$ Santa Cruz Institute for Particle Physics, Department of Physics and Department of Astronomy and Astrophysics, University of California at Santa Cruz, Santa Cruz, CA 95064, USA

${ }^{12}$ Università degli Studi di Pavia, I-27100 Pavia, Italy

${ }^{13}$ INAF-Istituto di Astrofisica Spaziale e Fisica Cosmica, I-20133 Milano, Italy

${ }^{14}$ Istituto Nazionale di Fisica Nucleare, Sezione di Pisa, I-56127 Pisa, Italy

${ }^{15}$ National Centre for Radio Astrophysics, Tata Institute of Fundamental Research, Pune 411 007, India

${ }^{16}$ Istituto Nazionale di Fisica Nucleare, Sezione di Trieste, and Università di Trieste, I-34127 Trieste, Italy

${ }^{17}$ Istituto Nazionale di Fisica Nucleare, Sezione di Perugia, I-06123 Perugia, Italy

${ }^{18}$ Dipartimento di Fisica, Università degli Studi di Perugia, I-06123 Perugia, Italy

${ }^{19}$ NASA Goddard Space Flight Center, Greenbelt, MD 20771, USA; ozlemceliktinmaz@ gmail.com

${ }^{20}$ Dipartimento di Fisica “M. Merlin” dell’Università e del Politecnico di Bari, I-70126 Bari, Italy

${ }^{21}$ Istituto Nazionale di Fisica Nucleare, Sezione di Bari, 70126 Bari, Italy

${ }^{22}$ Laboratoire Leprince-Ringuet, École polytechnique, CNRS/IN2P3, Palaiseau, France

${ }^{23}$ Deutsches Elektronen Synchrotron DESY, D-15738 Zeuthen, Germany

${ }^{24}$ INAF - Cagliari Astronomical Observatory, I-09012 Capoterra (CA), Italy

${ }^{25}$ Department of Physics, University of Washington, Seattle, WA 98195-1560, USA

${ }^{26}$ Institut de Ciències de l'Espai (IEEE-CSIC), Campus UAB, E-08193 Barcelona, Spain

${ }^{27}$ Columbia Astrophysics Laboratory, Columbia University, New York, NY 10027, USA ${ }^{28}$ Arecibo Observatory, Arecibo, PR 00612, USA

${ }^{29}$ Center for Research and Exploration in Space Science and Technology (CRESST) and NASA Goddard Space Flight Center, Greenbelt, MD 20771, USA

${ }^{30}$ Department of Physics and Center for Space Sciences and Technology, University of Maryland Baltimore County, Baltimore, MD 21250, USA

${ }^{31}$ Agenzia Spaziale Italiana (ASI) Science Data Center, I-00044 Frascati (Roma), Italy

32 Istituto Nazionale di Astrofisica - Osservatorio Astronomico di Roma, I-00040 Monte Porzio Catone (Roma), Italy 
${ }^{33}$ Laboratoire de Physique et Chimie de l'Environnement, LPCE UMR 6115 CNRS, F-45071 Orléans Cedex 02, and Station de radioastronomie de Nançay, Observatoire de Paris, CNRS/INSU, F-18330 Nançay, France

${ }^{34}$ Laboratoire Univers et Particules de Montpellier, Université Montpellier 2, CNRS/IN2P3, Montpellier, France

${ }^{35}$ Department of Physics and Astronomy, Sonoma State University, Rohnert Park, CA 94928-3609, USA

${ }^{36}$ Department of Physics, Stockholm University, AlbaNova, SE-106 91 Stockholm, Sweden

37 The Oskar Klein Centre for Cosmoparticle Physics, AlbaNova, SE-106 91 Stockholm, Sweden

38 The Royal Swedish Academy of Sciences, Box 50005, SE-104 05 Stockholm, Sweden

${ }^{39}$ INAF Istituto di Radioastronomia, 40129 Bologna, Italy

${ }^{40}$ Dipartimento di Fisica, Università di Udine and Istituto Nazionale di Fisica Nucleare, Sezione di Trieste, Gruppo Collegato di Udine, I-33100 Udine, Italy

${ }^{41}$ Department of Physics and Department of Astronomy, University of Maryland, College Park, MD 20742, USA

42 Istituto Universitario di Studi Superiori (IUSS), I-27100 Pavia, Italy

${ }^{43}$ Space Science Division, Naval Research Laboratory, Washington, DC 20375-5352, USA

${ }^{44}$ Max-Planck-Institut für Radioastronomie, Auf dem Hügel 69, D-53121 Bonn, Germany

${ }^{45}$ Centre d'Études Nucléaires de Bordeaux Gradignan, IN2P3/CNRS, Université Bordeaux 1, BP120, F-33175 Gradignan Cedex, France; smith@ cenbg.in2p3.fr

46 Jodrell Bank Centre for Astrophysics, School of Physics and Astronomy, The University of Manchester, M13 9PL, UK ${ }^{47}$ CNRS, IRAP, F-31028 Toulouse cedex 4, France

${ }^{48}$ GAHEC, Université de Toulouse, UPS-OMP, IRAP, Toulouse, France

${ }^{49}$ Department of Physical Sciences, Hiroshima University, Higashi-Hiroshima, Hiroshima 739-8526, Japan

${ }^{50}$ Department of Astronomy, Graduate School of Science, Kyoto University, Sakyo-ku, Kyoto 606-8502, Japan

${ }^{51}$ Netherlands Institute for Radio Astronomy (ASTRON), Postbus 2, 7990 AA Dwingeloo, Netherlands

52 Astronomical Institute "Anton Pannekoek” University of Amsterdam, Postbus 942491090 GE Amsterdam, Netherlands

${ }^{53}$ School of Physics and Astronomy, University of Southampton, Highfield, Southampton, SO17 1BJ, UK

${ }^{54}$ Department of Physics, Center for Cosmology and Astro-Particle Physics, The Ohio State University, Columbus, OH 43210, USA

${ }^{55}$ Department of Physics, Royal Institute of Technology (KTH), AlbaNova, SE-106 91 Stockholm, Sweden

${ }^{56}$ Science Institute, University of Iceland, IS-107 Reykjavik, Iceland

${ }^{57}$ National Research Council Research Associate, National Academy of Sciences, Washington, DC 20001, USA

${ }^{58}$ CSIRO Astronomy and Space Science, Australia Telescope National Facility, Epping NSW 1710, Australia

${ }^{59}$ Research Institute for Science and Engineering, Waseda University, 3-4-1, Okubo, Shinjuku, Tokyo 169-8555, Japan

${ }^{60}$ Department of Astronomy, Stockholm University, SE-106 91 Stockholm, Sweden

${ }^{61}$ Istituto Nazionale di Fisica Nucleare, Sezione di Torino, I-10125 Torino, Italy

${ }^{62}$ Department of Physics, West Virginia University, Morgantown, WV 26506, USA

${ }^{63}$ Mullard Space Science Laboratory, University College London, Holmbury St. Mary, Dorking, Surrey, RH5 6NT, UK

${ }^{64}$ Kepler Institute of Astronomy, University of Zielona Gra, Lubuska 2, 65-265, Zielona Gra, Poland

${ }^{65}$ Hiroshima Astrophysical Science Center, Hiroshima University, Higashi-Hiroshima, Hiroshima 739-8526, Japan

${ }^{66}$ Istituto Nazionale di Fisica Nucleare, Sezione di Roma “Tor Vergata," I-00133 Roma, Italy

${ }^{67}$ Yamagata University, 1-4-12 Kojirakawa-machi, Yamagata-shi, 990-8560, Japan

${ }^{68}$ Department of Physics and Astronomy, University of Denver, Denver, CO 80208, USA

${ }^{69}$ Max-Planck-Institut für Physik, D-80805 München, Germany

${ }^{70}$ Albert-Einstein-Institut, Max-Planck-Institut für Gravitationsphysik, D-30167 Hannover, Germany

${ }^{71}$ Leibniz Universität Hannover, D-30167 Hannover, Germany

72 National Radio Astronomy Observatory (NRAO), Charlottesville, VA 22903, USA

${ }^{73}$ Institut für Astro- und Teilchenphysik and Institut für Theoretische Physik, Leopold-Franzens-Universität Innsbruck, A-6020 Innsbruck, Austria

${ }^{74}$ Department of Physics, Washington University, St. Louis, MO 63130, USA

${ }^{75}$ Space Sciences Division, NASA Ames Research Center, Moffett Field, CA 94035-1000, USA

${ }^{76}$ NYCB Real-Time Computing Inc., Lattingtown, NY 11560-1025, USA

${ }^{77}$ Max-Planck Institut für extraterrestrische Physik, D-85748 Garching, Germany

${ }^{78}$ Department of Chemistry and Physics, Purdue University Calumet, Hammond, IN 46323-2094, USA

79 Department of Physics, Willamette University, Salem, OR 97031, USA

${ }^{80}$ Institut für Theoretische Physik and Astrophysik, Universität Würzburg, D-97074 Würzburg, Germany

${ }^{81}$ Institució Catalana de Recerca i Estudis Avançats (ICREA), Barcelona, Spain

${ }^{82}$ Rikkyo University, 3-34-1 Nishi-Ikebukuro, Toshima-ku, Tokyo 171-8501, Japan

${ }^{83}$ Centre for Space Research, North-West University, Potchefstroom Campus, Private Bag X6001, 2520 Potchefstroom, South Africa

${ }^{84}$ Consorzio Interuniversitario per la Fisica Spaziale (CIFS), I-10133 Torino, Italy

${ }^{85}$ Dipartimento di Fisica, Università di Roma “Tor Vergata," I-00133 Roma, Italy

${ }^{86}$ Urumqi Observatory, NAOC, Xinjiang 830011, China

${ }^{87}$ Praxis Inc., Alexandria, VA 22303, USA

Received 2013 May 7; accepted 2013 June 29; published 2013 September 19

\section{ABSTRACT}

This catalog summarizes 117 high-confidence $\geqslant 0.1 \mathrm{GeV}$ gamma-ray pulsar detections using three years of data acquired by the Large Area Telescope (LAT) on the Fermi satellite. Half are neutron stars discovered using LAT data through periodicity searches in gamma-ray and radio data around LAT unassociated source positions. The 117 pulsars are evenly divided into three groups: millisecond pulsars, young radio-loud pulsars, and young radio-quiet pulsars. We characterize the pulse profiles and energy spectra and derive luminosities when distance information exists. Spectral analysis of the off-peak phase intervals indicates probable pulsar wind nebula emission for four pulsars, and off-peak magnetospheric emission for several young and millisecond pulsars. We compare the gammaray properties with those in the radio, optical, and X-ray bands. We provide flux limits for pulsars with no observed gamma-ray emission, highlighting a small number of gamma-faint, radio-loud pulsars. The large, varied gamma-ray pulsar sample constrains emission models. Fermi's selection biases complement those of radio surveys, enhancing comparisons with predicted population distributions.

Key words: catalogs - pulsars: general - stars: neutron 


\section{INTRODUCTION}

Pulsars have featured prominently in the gamma-ray sky since the birth of gamma-ray astronomy. The Crab and Vela pulsars were the first two sources identified in the 1970's by SAS-2 (Fichtel et al. 1975) and COS-B (Swanenburg et al. 1981). In the 1990's the Compton Gamma-Ray Observatory brought the pulsar grand total to at least seven, along with three other strong candidates (Thompson 2008). One of these early gammaray pulsars, Geminga, was undetected at radio wavelengths (Bignami \& Caraveo 1996). Despite the meager number, neutron stars were estimated to represent a sizeable fraction of the EGRET unassociated low-latitude gamma-ray sources (Romani \& Yadigaroglu 1995). The Large Area Telescope (LAT) on the Fermi Gamma-Ray Space Telescope did not just confirm the expectation: by discovering dozens of radio-quiet gamma-ray pulsars and millisecond pulsars (MSPs; thought to be old pulsars spun up to rapid periods via accretion from a companion, Alpar et al. 1982), the LAT established pulsars as the dominant $\mathrm{GeV}$ gamma-ray source class in the Milky Way (Abdo et al. 2010m; The First Fermi Large Area Telescope Catalog of Gamma-ray Pulsars, hereafter 1PC).

A pulsar is a rapidly-rotating, highly-magnetized neutron star, surrounded by a plasma-filled magnetosphere. Modeling its emission drives ever-more sophisticated electrodynamic calculations (e.g., Wang \& Hirotani 2011; Li et al. 2012; Kalapotharakos et al. 2012b; Pétri 2012). Throughout this paper, we will call pulsars in the main population of the spin period $(P)$ and period derivative $(\dot{P})$ plane "young" to distinguish them from the much older "recycled" pulsars, including MSPs. All known gamma-ray pulsars, and the most promising candidates to date, are rotation-powered pulsars (RPPs). The LAT has yet to detect significant gamma-ray pulsations from any accretionpowered pulsar or from the magnetars, anomalous X-ray pulsars, and soft gamma repeaters for which the dominant energy source is not electromagnetic braking, but magnetic field decay (Parent et al. 2011).

Here we present 117 gamma-ray pulsars unveiled in $3 \mathrm{yr}$ of on-orbit observations with Fermi. Extensive radio observations by the "Pulsar Timing Consortium" (Smith et al. 2008) greatly enhanced the gamma-ray data analysis. Our analysis of the gamma-ray pulsars is as uniform as is feasible given the widely varying pulsar characteristics. In addition to $1 \mathrm{PC}$, this catalog builds on the Second Fermi LAT source catalog (Nolan et al. 2012, hereafter 2FGL), which reported pulsations for 83 of the 2FGL sources, included here. An additional 27 pulsars were found to be spatially associated with $2 \mathrm{FGL}$ sources and pulsations have since been established for 12 of these, included here. The remaining 22 new pulsars with strong pulsations were either unassociated in 2FGL (pointing to subsequent pulsar discoveries; see Section 3) or were below the 2FGL detection threshold and seen to pulse after 2FGL was completed.

\footnotetext{
${ }^{88}$ Resident at Naval Research Laboratory, Washington, DC 20375, USA.

${ }^{89}$ Royal Swedish Academy of Sciences Research Fellow, funded by a grant from the K. A. Wallenberg Foundation.

${ }^{90}$ NASA Postdoctoral Program Fellow, USA.

${ }^{91}$ Funded by a Marie Curie IOF, FP7/2007-2013 — Grant agreement No. 275861.

92 Funded by contract ERC-StG-259391 from the European Community.
}

We provide our results in FITS $^{93}$ and image files, with hyperlinks from this article to the journal's servers, and available as well as on the Fermi Science Support Center (FSSC) servers at http://fermi.gsfc.nasa.gov/ssc/data/access/lat/2nd PSR_catalog/. The structure of the paper is as follows. Section 2, "Observations," describes the instrument and data sample. Section 3 explains pulsation discovery methods, and in Section 4 we list the gamma-ray pulsars, with some key properties. Section 5, "Profile Characterization," describes our fits to the lightcurves, and Section 6 details the spectral analyses. Section 7 is called "Unpulsed Magnetospheric Emission," an analysis of the phase intervals away from the gamma-ray peaks. Section 8 focuses on candidates for pulsed gamma-ray emission that we have not presently detected. Section 9 lists optical and $\mathrm{X}$-ray measurements of the pulsar sample. Discussion of our results is in Section 10. Three appendices follow: the first with a sample of the light curves and spectra (the complete set being provided online), the second detailing the online version of the catalog, and the last discussing individual pulsars highlighted in the off-peak analysis of Section 7.

\section{OBSERVATIONS}

Fermi was launched on 2008 June 11, carrying two gammaray instruments: the LAT and the Gamma-ray Burst Monitor (Meegan et al. 2009); the latter was not used to prepare this catalog. Atwood et al. (2009) describe Fermi's main instrument, the LAT, and on-orbit performance of the LAT is reported by Abdo et al. (2009f) and Ackermann et al. (2012a). The LAT is a pair-production telescope composed of a $4 \times 4$ grid of towers. Each tower consists of a stack of tungsten foil converters interleaved with silicon-strip particle tracking detectors, mated with a hodoscopic cesium-iodide calorimeter. A segmented plastic scintillator anti-coincidence detector covers the grid to help discriminate charged particle backgrounds from gammaray photons. The LAT field of view is $\sim 2.4 \mathrm{sr}$. The primary operational mode is a sky survey where the satellite rocks between a pointing above the orbital plane and one below the plane after each orbit. The entire sky is imaged every two orbits $(\sim 3 \mathrm{hr}$ ) and any given point on the sky is observed $\sim 1 / 6$ of the time. Each event classified as a gamma ray in the ground data processing has its incident direction, incident energy $(E)$, and time of arrival recorded in the science data stream.

The LAT is sensitive to gamma rays with energies $E$ from $20 \mathrm{MeV}$ to over $300 \mathrm{GeV}$, with an on-axis effective area of $\sim 8000 \mathrm{~cm}^{2}$ above $1 \mathrm{GeV}$. Multiple Coulomb scattering of the electron-positron pairs created by converted gamma rays degrades the per-photon angular resolution with decreasing energy as $\theta_{68}^{2}(E)=(3.3)^{2}(100 \mathrm{MeV} / E)^{1.56}+(0.1)^{2}$, averaged over the acceptance for events converting in the front section of the LAT, where $\theta_{68}$ is the $68 \%$ containment radius. The energy- and direction-dependent effective area and point-spread function (PSF) are part of the Instrument Response Functions (IRFs). The analysis in this paper used the Pass7 V6 IRFs selecting events in the "Source" class (Ackermann et al. 2012a).

The data used here to search for gamma-ray pulsars span 2008 August 4 to 2011 August 4. Events were selected with reconstructed energies from 0.1 to $100 \mathrm{GeV}$ and directions within $2^{\circ}$

\footnotetext{
93 http://fits.gsfc.nasa.gov/
} 
of each pulsar position for pulsation searches (Section 3) and $15^{\circ}$ for spectral analyses (Section 6 ). We excluded gamma rays collected when the LAT was not in nominal science operations mode or the spacecraft rocking angle exceeded $52^{\circ}$, as well as those with measured zenith angles $>100^{\circ}$, to greatly reduce the residual gamma rays from the bright limb of the Earth. For PSRs J0205+6449, J1838-0537, and J2215+5135 we did not have timing solutions that were coherent over the full 3 yr. For these pulsars the data sets for pulsation searches and light curve generation only include events within the validity range of the corresponding timing solutions. For the first two pulsars, the data loss is $<7 \%$ but for PSR J2215+5135 it is $60 \%$.

\section{PULSATION DISCOVERY}

Events recorded by the LAT have timestamps derived from GPS clocks integrated into the satellite's Guidance, Navigation, and Control (GNC) subsystem, accurate to $<1 \mu$ s relative to UTC (Abdo et al. 2009f). The GNC subsystem provides the instantaneous spacecraft position with corresponding accuracy. We compute pulsar rotational phases $\phi_{i}$ using TEMPO2 (Hobbs et al. 2006) with the fermi plug-in (Ray et al. 2011). The fermi plug-in uses the recorded times and spacecraft positions combined with a pulsar timing ephemeris (specified in a TEMPO2 parameter, or "par," file). The par files are all provided in the online material. The timing chain from the instrument clocks through the barycentering and epoch folding software is accurate to better than a few $\mu$ s for binary orbits, and significantly better for isolated pulsars (Smith et al. 2008). The accuracy of the phase computation is thus determined by the ephemeris. The par file is created using radio or gamma-ray data, or a mix, depending on the LAT pulsar discovery method, as described in the following three subsections.

We required $a \geqslant 5 \sigma$ confidence level detection of modulation in the phase histogram for a pulsar to be included in this catalog, as described below. Gamma-ray pulsar data are extremely sparse, often with fewer than one photon detected in tens of thousands (or in the case of MSPs, millions!) of pulsar rotations. In these circumstances, the favored techniques are unbinned tests for periodic signals. We use the $H$-test (de Jager et al. 1989; de Jager \& Büsching 2010), a statistical test for discarding the null hypothesis that a set of photon phases is uniformly distributed. For $N_{\gamma}$ gamma-rays, the $H$-test statistic is $H \equiv \max \left(Z_{m}^{2}-4 \times(m-1), 1 \leqslant m \leqslant 20\right)$, with $Z_{m}^{2} \equiv$ $\left(2 / N_{\gamma}\right) \sum_{k=1}^{m} \alpha_{k}^{2}+\beta_{k}^{2}$, and $\alpha_{k}$ and $\beta_{k}$ the empirical trigonometric coefficients $\alpha_{k} \equiv \sum_{i=1}^{N_{\gamma}} \sin \left(2 \pi k \phi_{i}\right)$ and $\beta_{k} \equiv \sum_{i=1}^{N_{\gamma}} \cos \left(2 \pi k \phi_{i}\right)$. By including a search over a range of harmonics, the $H$-test maintains sensitivity to light curves with a large range of morphologies (e.g., sharp versus broad peaks). The sharpness of the peaks in the gamma-ray profile has a large impact on the detectability of the pulsar; in particular, pulsars with narrow, sharp peaks are easier to detect than pulsars with broad peaks covering more of the pulse phase.

Early in the mission most pulsation searches (for example, Abdo et al. 2009b) selected events with arrival directions within a fixed angular distance of the pulsar (the region of interest, or ROI) and a minimum energy cut $\left(E_{\min }\right)$. Because of the range of pulsar spectra, fluxes, and levels of diffuse gamma-ray background, combined with the strongly energy-dependent PSF of the LAT, a number of trials must be done over a range of ROI sizes and $E_{\min }$ to optimize the detection significance for each candidate pulsar.
Using the probability that each event originates from the pulsar, computed from a spectral model of the region and the LAT IRFs, the $H$-test can be extended, using these probabilities as weights (Kerr 2011). This both improves the sensitivity of the $H$-test and removes the need for trials over event selection criteria. The weights, $w_{i}$, representing the probability that the $i$ th event originates from the pulsar are

$$
w_{i}=\frac{d N / d E_{\mathrm{psr}}\left(E_{i}, \boldsymbol{x}_{i}\right)}{\sum_{j} d N / d E_{j}\left(E_{i}, \boldsymbol{x}_{i}\right)},
$$

where $E_{i}$ and $\boldsymbol{x}_{i}$ are the observed energy and position on the sky of the $i$ th event and $d N / d E_{j}$ is the phase-averaged spectra for the $j$ th source in the ROI (see Section 6). Incorporating the weights yields the weighted $H$-test, $H \equiv \max \left(Z_{\mathrm{mw}}^{2}-4 \times(m-1)\right.$; $1 \leqslant m \leqslant 20$ ), with

$$
Z_{\mathrm{mw}}^{2} \equiv \frac{2}{N_{\gamma}}\left(\frac{1}{N_{\gamma}} \sum_{i=1}^{N_{\gamma}} w_{i}^{2}\right)^{-1} \sum_{k=1}^{m} \alpha_{\mathrm{kw}}^{2}+\beta_{\mathrm{kw}}^{2}
$$

where $\alpha_{\mathrm{kw}}=\sum_{i=1}^{N_{\gamma}} w_{i} \cos \left(2 \pi k \phi_{i}\right)$ and $\beta_{\mathrm{kw}}=\sum_{i=1}^{N_{\gamma}} w_{i}$ $\sin \left(2 \pi k \phi_{i}\right)$. Kerr (2011) provides the probability that a detection is a statistical fluctuation for a given $H$ value, approximated by $e^{-0.4 H} . H=36(15)$ corresponds to a $5 \sigma(3 \sigma)$ detection.

\subsection{Using Known Rotation Ephemerides}

The first gamma-ray pulsar discovery method, described above, found 61 of the gamma-ray pulsars in this catalog. It uses known rotational ephemerides from radio or X-ray observatories. The 2286 known RPPs (mostly from the ATNF Pulsar Catalog ${ }^{94}$ Manchester et al. 2005; see Table 1) are all candidate gamma-ray pulsars. Nearly all of these were discovered in radio searches, with a handful coming from X-ray observations. Phase-folding with a radio or X-ray ephemeris is the most sensitive way to find gamma-ray pulsations, since no penalties are incurred for trials in position, $P, \dot{P}$, or other search parameters. Having a current ephemeris for as many known pulsars as possible is of critical importance to LAT science and is the key goal of the Pulsar Timing Consortium (Smith et al. 2008). EGRET results (Thompson 2008) as well as theoretical expectations indicated that young pulsars with large spindown power, ${ }^{95} \dot{E}>1 \times 10^{34} \mathrm{erg} \mathrm{s}^{-1}$, are the most likely gamma-ray pulsar candidates. Because of their intrinsic instabilities, such as timing noise and glitches, these pulsars are also the most resource intensive to maintain ephemerides of sufficient accuracy. To allow for unexpected discoveries, the Timing Consortium also provides ephemerides for essentially all known pulsars that are regularly timed, spanning the $P \ddot{P}$ space of known pulsars (Figure 1). In addition to $\dot{E}$, the $P \dot{P}$ diagram shows two other physical parameters derived from the timing information: the magnetic field at the neutron star surface, $B_{\mathrm{S}}=\left(1.5 I_{0} c^{3} P \dot{P}\right)^{1 / 2} / 2 \pi R_{\mathrm{NS}}^{3}$, assuming an orthogonal rotator with neutron star radius $R_{\mathrm{NS}}=10 \mathrm{~km}$ and the speed of light in a vacuum, $c$; and the characteristic age $\tau_{c}=P / 2 \dot{P}$, assuming magnetic dipole braking as the only energy-loss mechanism and an initial spin period much less than the current period. The black dots in Figure 1 show 710 pulsars that we have phasefolded without detecting gamma pulsations, in addition to the 117 gamma-ray pulsars. The locations of all 117 gamma-ray pulsars on the sky are shown in Figure 2.

\footnotetext{
94 http://www.atnf.csiro.au/research/pulsar/psrcat

$95 \dot{E}=4 \pi^{2} I_{0} \dot{P} / P^{3}$, for which we use $I_{0}=10^{45} \mathrm{~g} \mathrm{~cm}^{2}$ as the neutron star moment of inertia.
} 


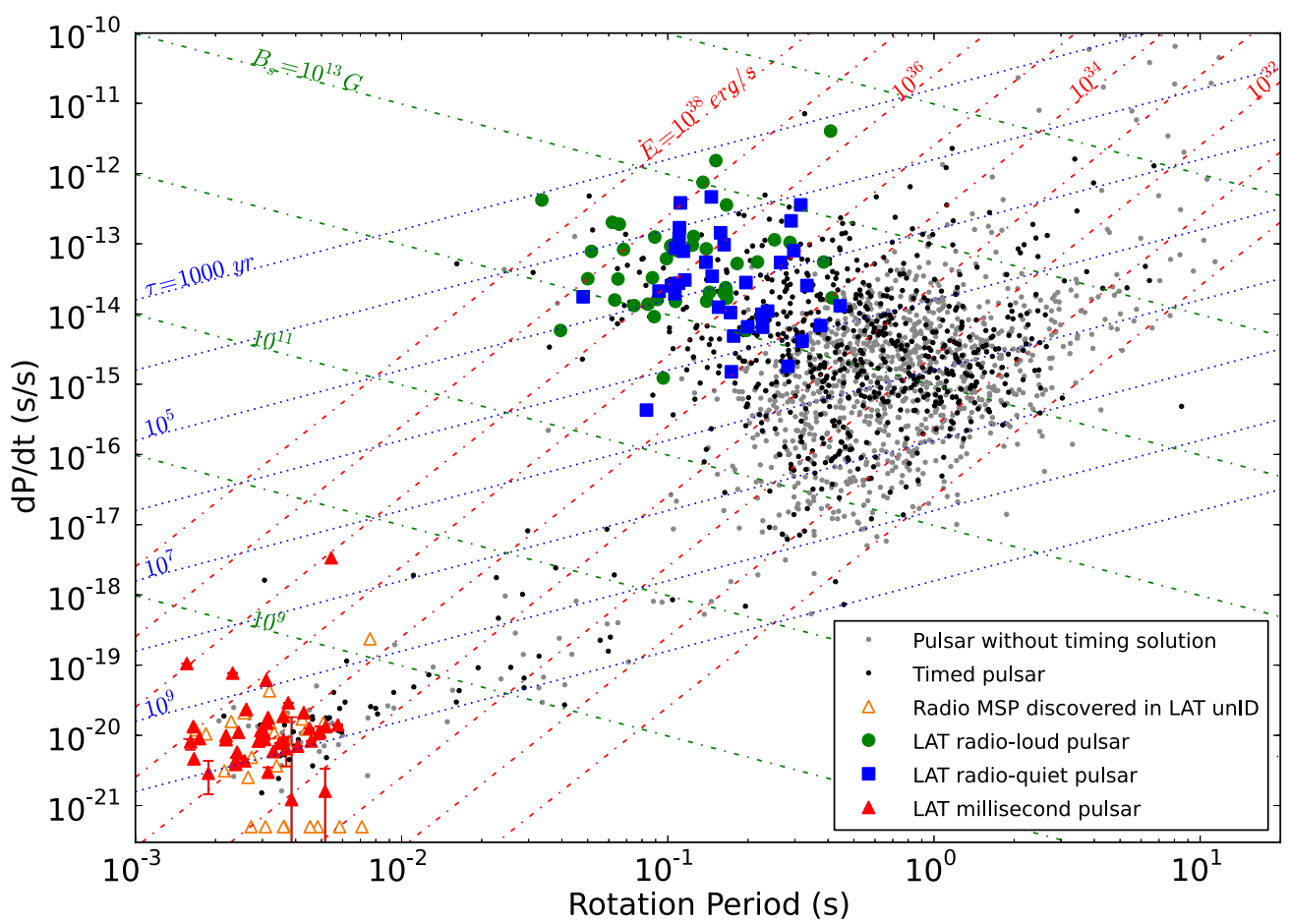

Figure 1. Pulsar spindown rate, $\dot{P}$, vs. the rotation period $P$. Green dots indicate the 42 young, radio-loud gamma-ray pulsars and blue squares show the 35 young, "radio-quiet" pulsars, defined as $S_{1400}<30 \mu \mathrm{Jy}$, where $S_{1400}$ is the radio flux density at $1400 \mathrm{MHz}$. Red triangles are the 40 millisecond gamma-ray pulsars. The 710 black dots indicate pulsars phase-folded in gamma rays using rotation models provided by the "Pulsar Timing consortium" for which no significant pulsations were observed. Phase-folding was not performed for the 1337 pulsars outside of globular clusters indicated by gray dots. Orange open triangles indicate radio MSPs discovered at the positions of previously unassociated LAT sources for which we have not yet seen gamma pulsations. We plot them at $\dot{P} \equiv 5 \times 10^{-22}$ when $\dot{P}$ is unavailable. Shklovskii corrections to $P$ have been applied to the pulsars with proper motion measurements (see Section 4.3). For clarity, error bars are shown only for the gamma-detected pulsars.

(A color version of this figure is available in the online journal.)

Table 1

Pulsar Varieties

\begin{tabular}{|c|c|c|c|}
\hline Category & Count & Sub-count & Fraction \\
\hline Known rotation-powered pulsars (RPPs) ${ }^{\mathrm{a}}$ & 2286 & & \\
\hline RPPs with measured $\dot{P}>0$ & & 1944 & \\
\hline RPPs with measured $\dot{E}>3 \times 10^{33} \mathrm{erg} \mathrm{s}^{-1}$ & & 552 & \\
\hline Millisecond pulsars (MSPs; $P<16 \mathrm{~ms}$ ) & 292 & & \\
\hline Field MSPs & & 169 & \\
\hline MSPs in globular clusters & & 123 & \\
\hline Field MSPs with measured $\dot{E}>3 \times 10^{33} \mathrm{erg} \mathrm{s}^{-1}$ & & 96 & \\
\hline Globular cluster MSPs with measured $\dot{E}>3 \times 10^{33} \mathrm{erg} \mathrm{s}^{-1}$ & & 25 & \\
\hline Gamma-ray pulsars in this catalog & 117 & & \\
\hline Young or middle-aged & & 77 & \\
\hline Radio-loud gamma-ray ${ }^{\mathrm{b}}$ & & 42 & $36 \%$ \\
\hline Radio-quiet gamma-ray & & 35 & $30 \%$ \\
\hline Gamma-ray MSPs (isolated + binary) & & $(10+30)=40$ & $34 \%$ \\
\hline Radio MSPs discovered in LAT sources & 46 & & \\
\hline with gamma-ray pulsations ${ }^{\mathrm{c}}$ & & 34 & \\
\hline
\end{tabular}

Notes.

a Includes the 2193 pulsars, which are all RPPs, in the ATNF Pulsar Catalog (v1.46, Manchester et al. 2005); see http://www.atnf.csiro.au/research/pulsar/psrcat, as well as more recent discoveries. D. Lorimer maintains a list of known field MSPs at http://astro.phys.wvu.edu/GalacticMSPs/.

${ }^{\text {b }} S_{1400}>30 \mu \mathrm{Jy}$, where $S_{1400}$ is the radio flux density at $1400 \mathrm{MHz}$.

${ }^{\mathrm{c}}$ Only 20 of the new radio MSPs showed gamma-ray pulsations when the dataset for this catalog was frozen.

For known pulsars we use years of radio and/or X-ray timeof-arrival measurements ("TOAs") to fit the timing model parameters using the standard pulsar timing codes TEMPO (Taylor \& Weisberg 1989) or TEMPO2 (Hobbs et al. 2006). In addition to providing a model for folding the gamma-ray data, the radio observations also provide the information needed to measure the absolute phase alignment (after correcting for interstellar dispersion) between the radio or X-ray and gamma-ray pulses, providing key information about the relative geometry of the different emission regions. 


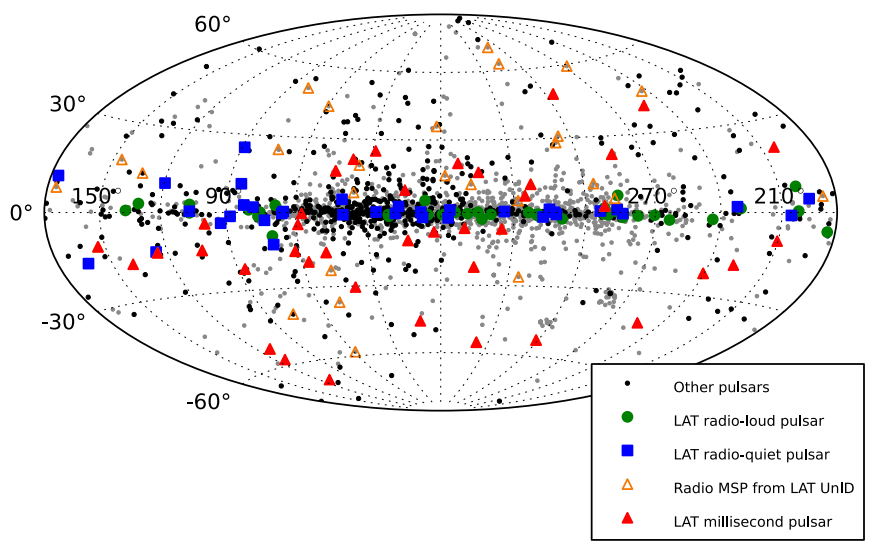

Figure 2. Pulsar sky map in Galactic coordinates. The markers are the same as in Figure 1.

(A color version of this figure is available in the online journal.)

\subsection{Blind Periodicity Searches}

The second method of discovering gamma-ray pulsars, which produced 36 (approximately one-third) of the gamma-ray pulsars in this catalog, involves detecting the rotational period in the LAT data. Both these searches and the radio searches described in the next subsection begin with a target list of candidate pulsars. Some targets are sources known at other wavelengths that are suspected of harboring pulsars. These include supernova remnants (SNRs), pulsar wind nebulae (PWNe), compact central objects (CCOs), unidentified TeV sources, and other high-energy sources, mostly along the Galactic plane. Generally, these sources had already been subjected to deep radio searches independent of Fermi.

In addition, as the LAT surveys the sky, an increasing number of gamma-ray sources are discovered and characterized that are not associated with previously known objects. Several methods have been used to rank these according to their probabilities of being yet-undiscovered pulsars. Most of these rely on the tendency of gamma-ray pulsars to be non-variable and have spectra that can be fit with exponential cutoffs in the few $\mathrm{GeV}$ band (Ackermann et al. 2012b; Lee et al. 2012).

Blind searches for pulsars in gamma rays are challenging, due to the wide pulsar parameter ranges that must be searched and due to the sparseness of the data (a few photons per hour for the brightest sources). This results in very long integration times (months to years) making standard Fast Fourier Transform search techniques computationally prohibitive. New semi-coherent search techniques (Atwood et al. 2006; Pletsch et al. 2012a) have been extremely successful at discovering gamma-ray pulsars with modest computational requirements.

LAT blind search sensitivity depends on a number of parameters: the rotation frequency, energy spectrum, pulsed fraction, level of diffuse gamma-ray background, event extraction choices (e.g., ROI and $E_{\min }$ ), and the accuracy of the position used to barycenter the data. The $1 \mathrm{yr}$ sensitivity was evaluated using a Monte Carlo study by Dormody et al. (2011). Newer searches (Pletsch et al. 2012a, 2012b) have mitigated dependence on event selection criteria and source localization by weighting events and searching over a grid of positions.

In all, well over one hundred LAT sources have been subjected to blind period searches. Pulsars might have been missed due to (1) low pulsed fraction or very high backgrounds, (2) broad pulse profiles (our algorithms detect sharp pulses more easily), (3) high levels of timing noise or glitches, or (4) being in an unknown binary system. Most MSPs are in binary systems, where the Doppler shifts from the orbital motion smear the signal. In some cases, multiwavelength observations constrain the orbit and position to make the search more like that of an isolated MSP. Optical studies (Romani \& Shaw 2011; Kong et al. 2012; Romani 2012) led to the first discovery of a millisecond pulsar, PSR J1311-3430, in a blind search of LAT data (Pletsch et al. 2012c). Detection of radio pulsations followed shortly (Ray et al. 2013). Even isolated MSP searches require massive computation with fine frequency and position gridding. The Einstein@ home $^{96}$ project applies the power of global volunteer computing to this problem.

For the LAT pulsars undetected in the radio (see Section 4.1), or too faint for regular radio timing, we must determine the pulsar timing ephemeris directly from the LAT data. Techniques for TOA determination optimized for sparse photon data have been developed and applied to generate the timing models required for the profile analysis (Ray et al. 2011). This timing provides much more precise pulsar positions than can be determined from the LAT event directions, which is important for multiwavelength counterpart searches. It also allows study of timing noise and glitch behavior.

\subsection{Radio Pulsar Discoveries Leading to Gamma-Ray Pulsations}

In the third discovery method that we applied, which yielded 20 of this catalog's MSPs, unassociated LAT source positions are searched for radio pulsations. When found, the resulting ephemeris enables gamma-ray phase-folding, as in Section 3.1. A key feature of radio pulsar searches is that they are sensitive to binary systems with the application of techniques to correct for the orbital acceleration in short data sets (with durations much less than the binary period, Ransom et al. 2002). This allows for the discovery of binary MSPs, which are largely inaccessible to gamma-ray blind searches, as described above.

Radio searches of several hundred LAT sources by the Fermi Pulsar Search Consortium (PSC), an international collaboration of radio observers with access to large radio telescopes, have resulted in the discovery of 47 pulsars, including 43 MSPs and four young or middle-aged pulsars (Ray et al. 2012). As the LAT Collaboration generates internal source lists and preliminary catalogs of gamma-ray sources from the accumulating sky-survey data, these target localizations are provided to the PSC for searching, with rankings of how strongly their characteristics resemble those of gamma-ray pulsars, as described in Section 3.2. This technique was employed during the EGRET era as well, but with modest success, in part due to the relatively poor source localizations. With the LAT, there are many more gamma-ray sources detected and each one is localized to an accuracy that is comparable to, or smaller than, the beam width of the radio telescopes being used. This enables deep searches by removing the need to mosaic a large region. It also facilitates repeated searches of the same source, which is important because discoveries can be missed as a result of scintillation or eclipses in binary systems (e.g., PSR J0101-6422, see Kerr et al. 2012).

Guided by these ranked lists of pulsar-like gamma-ray sources, the 43 radio MSPs were discovered in a tiny fraction of the radio telescope time that would have been required to find them in undirected radio pulsar surveys. In particular, because the MSP population out to the LAT's detection limit $(\sim 2 \mathrm{kpc})$ is distributed nearly uniformly across the sky, full sky

\footnotetext{
96 http://einstein.phys.uwm.edu
} 
surveys are required, whereas most young pulsar searches have concentrated on the Galactic plane. For comparison, after analyzing thousands of pointings carried out since 2007, the High Time Resolution Universe surveys (Keith 2012; Ng \& HTRU Collaboration 2013, and references therein) found 29 new radio MSPs.

Interestingly, the success rate for radio searches of LAT sources in the plane has been much poorer. Only four young pulsars have been discovered, and only one of those turned out to be a gamma-ray pulsar (PSR J2030+3641, Camilo et al. 2012), the others being chance associations. This is probably due to a combination of young pulsars having smaller radio beaming fractions than MSPs (as evidenced by the large number of young, radio-quiet pulsars discovered) and the fact that the Galactic plane has been well surveyed for radio pulsars. The great success of the blind gamma searches in the plane is because young pulsars mainly reside there. Their smaller radio beaming fractions leave a large number of radio-quiet pulsars that can only be discovered in high-energy data.

Once a radio pulsar has been discovered positionally coincident with a LAT source, it must be observed for a substantial period (typically six months to a year or more) to determine a timing model that allows a periodicity search in the LAT data, as described in Section 3.1. In several cases, an initial radio model has allowed discovery of the gamma-ray pulsations, then the LAT data themselves have been used to extend the validity of the timing model back through the launch of Fermi, a few years before the radio discovery. This radio follow up has resulted in the confirmed detection of LAT pulsations from 20 of these MSPs. Five more were detected using data beyond the set described in Section 2. Of the remainder, most will have LAT pulsations detected once their radio timing models are well determined, but a few (e.g., PSR J1103-5403; see Keith et al. 2011) are likely to be just chance coincidences with the target LAT source.

\section{THE GAMMA-RAY PULSARS}

The discovery strategies discussed in Section 3 yielded 117 gamma-ray pulsars in three years of data. Of the gamma-ray pulsars in this catalog, roughly half (41 young and 20 MSPs) were known in radio and/or X-rays prior to the launch of Fermi. The remaining pulsars were discovered by or with the aid of the LAT, with 36 being young pulsars found in blind searches of LAT data and the remaining being MSPs found in radio searches of unassociated LAT sources. Fermi has not only significantly increased the number of known energetic young and millisecond pulsars, but has done so with selection biases complementary to those of previous surveys. The LAT all-sky survey has its greatest sensitivity in regions of the sky away from the Galactic plane (see Section 8.2), increasing the diversity and the uniformity of the sampled neutron star population. As an example, Figure 2 shows the broad range of Galactic latitude of the Fermi pulsars.

Table 1 summarizes the census of known pulsars, independent of the method by which the pulsars were discovered. Tables 2 and 3 list the characteristics of the 117 gamma-ray pulsars, divided into young and millisecond gamma-ray pulsars, respectively. All have large spindown powers, $\dot{E}>3 \times 10^{33} \mathrm{erg} \mathrm{s}^{-1}$, apparent in Figure 1. The large uncertainties on the two seeming exceptions, PSRs J0610-2100 and J1024-0719, are discussed in Section 6.3.
Pulsar discoveries continue as increased statistics bring light curves above our $5 \sigma$ detection threshold, improved methods for event selection and blind searches allow increased sensitivity, and multiwavelength studies either detect radio pulsations or constrain the blind-search space for likely pulsar candidates. Table 4 lists a number of LAT pulsars announced since the sample was frozen for the uniform analysis of the present paper.

\subsection{Radio Intensities}

The $1400 \mathrm{MHz}$ flux densities, $S_{1400}$, of the young LATdetected pulsars are listed in Table 2, and in Table 3 for the MSPs. Figure 3 shows how they compare with the overall pulsar population. Whenever possible, we report $S_{1400}$ as given in the ATNF Pulsar Catalog. For radio-loud pulsars with no published value at $1400 \mathrm{MHz}$, we extrapolate to $S_{1400}$ from measurements at other frequencies, assuming $S_{v} \propto v^{\alpha}$, where $\alpha$ is the spectral index. For most pulsars $\alpha$ has not been measured, and we use an average value $\langle\alpha\rangle=-1.7$, a middle ground between -1.6 from Lorimer et al. (1995) and -1.8 from Maron et al. (2000). For those pulsars with measured spectral indices, we use the published value of $\alpha$ for the extrapolation. In the table notes, we list those pulsars for which we have extrapolated $S_{1400}$ from another frequency and/or used a value of $\alpha$ other than -1.7 for the extrapolation.

Table 2 also reports upper limits on $S_{1400}$ for blind search pulsars that have been observed, but not detected, at radio frequencies. We define these upper limits as the sensitivity of the observation given by the pulsar radiometer equation (Equation (7.10) on page 174 of Lorimer \& Kramer 2004) assuming a minimum signal-to-noise ratio of 5 for a detection and a pulse duty cycle of $10 \%$. We mention here the unconfirmed radio detections of Geminga and PSR J1732-3131 at low radio frequencies, consistent with their non-detection above $300 \mathrm{MHz}$ (Malofeev \& Malov 1997; Maan et al. 2012).

All pulsars discovered in blind searches have been searched deeply for radio pulsations (Saz Parkinson et al. 2010; Ray et al. 2011, 2012), and four of the 36 have been detected (Camilo et al. 2009; Abdo et al. 20101; Pletsch et al. 2012a). In 1PC we labeled the young pulsars by how they were discovered (radioselected versus gamma-ray selected), whereas we now define a pulsar as "radio-loud" if $S_{1400}>30 \mu \mathrm{Jy}$, and "radio-quiet" if the measured flux density is lower, as for the two pulsars with detections of very faint radio pulsations, or if no radio detection has been achieved. The horizontal line in Figure 3 shows the threshold. This definition favors observational characteristics instead of discovery history. Of the four radio-detected blindsearch pulsars, two remain radio-quiet whereas the other two could in principle have been discovered in a sensitive radio survey. The diagonal line in Figure 3 shows a possible alternate threshold at pseudo-luminosity $100 \mu \mathrm{Jy}-\mathrm{kpc}^{2}$, for reference. Three of the four have pseudo-luminosities lower than for any previously known young pulsar, and comparable to only a small number of MSPs.

\subsection{Distances}

Converting measured pulsar fluxes to emitted luminosities $L_{\gamma}$ (detailed in Section 6.3) requires the distances to the sources. Knowing the distances also allows mapping neutron star distributions relative to the Galaxy's spiral arms, as in Figure 4, or evaluating their scale height above the plane. Several methods can be used to estimate pulsar distances; however, the methods vastly differ in reliability. Deciding which method to 
Table 2

Some Parameters of Young LAT-detected Pulsars

\begin{tabular}{|c|c|c|c|c|c|c|c|}
\hline PSR & History & $\begin{array}{c}l \\
\left({ }^{\circ}\right)\end{array}$ & $\begin{array}{c}b \\
\left({ }^{\circ}\right)\end{array}$ & $\begin{array}{c}P \\
(\mathrm{~ms})\end{array}$ & $\begin{array}{c}\dot{P} \\
\left(10^{-15}\right) \\
\end{array}$ & $\begin{array}{c}\dot{E} \\
\left(10^{34} \mathrm{erg} \mathrm{s}^{-1}\right)\end{array}$ & $\begin{array}{l}S_{1400} \\
(\mathrm{mJy})\end{array}$ \\
\hline $\mathrm{J} 0007+7303$ & $\mathrm{~g}$ & 119.66 & 10.46 & 315.9 & 357. & 44.8 & $<0.005^{1}$ \\
\hline J0106+4855 & gu & 125.47 & -13.87 & 83.2 & 0.43 & 2.9 & 0.008 \\
\hline J0205+6449 & $\mathrm{x}$ & 130.72 & 3.08 & 65.7 & 190. & 2644. & 0.045 \\
\hline J0248+6021 & $\mathrm{r}$ & 136.90 & 0.70 & 217.1 & 55.0 & 21.2 & 13.7 \\
\hline $\mathrm{J} 0357+3205$ & $\mathrm{gu}$ & 162.76 & -16.01 & 444.1 & 13.1 & 0.6 & $<0.004^{1}$ \\
\hline J0534+2200 & re & 184.56 & -5.78 & 33.6 & 420. & 43606. & 14.0 \\
\hline J0622+3749 & $\mathrm{gu}$ & 175.88 & 10.96 & 333.2 & 25.4 & 2.7 & $<0.012^{2}$ \\
\hline J0631+1036 & $\mathrm{r}$ & 201.22 & 0.45 & 287.8 & 104. & 17.3 & 0.8 \\
\hline $\mathrm{J} 0633+0632$ & $\mathrm{gu}$ & 205.09 & -0.93 & 297.4 & 79.6 & 11.9 & $<0.003^{1}$ \\
\hline J0633+1746 & xe & 195.13 & 4.27 & 237.1 & 11.0 & 3.3 & $<0.507^{3}$ \\
\hline J0659+1414 & $\mathrm{r}$ & 201.11 & 8.26 & 384.9 & 55.0 & 3.8 & 3.7 \\
\hline J0729-1448 & $\mathrm{r}$ & 230.39 & 1.42 & 251.7 & 114. & 28.2 & 0.7 \\
\hline J0734-1559 & $\mathrm{gu}$ & 232.06 & 2.02 & 155.1 & 12.5 & 13.2 & $<0.005^{4}$ \\
\hline J0742-2822 & $\mathrm{r}$ & 243.77 & -2.44 & 166.8 & 16.8 & 14.3 & 15.0 \\
\hline J0835-4510 & re & 263.55 & -2.79 & 89.4 & 125. & 690. & 1100. \\
\hline J0908-4913 & $\mathrm{r}$ & 270.27 & -1.02 & 106.8 & 15.1 & 49.0 & 10.0 \\
\hline J0940-5428 & $\mathrm{r}$ & 277.51 & -1.29 & 87.6 & 32.8 & 193. & 0.66 \\
\hline J1016-5857 & $\mathrm{r}$ & 284.08 & -1.88 & 107.4 & 80.6 & 257. & 0.46 \\
\hline J1019-5749 & $\mathrm{r}$ & 283.84 & -0.68 & 162.5 & 20.1 & 18.4 & 0.8 \\
\hline J1023-5746 & gu & 284.17 & -0.41 & 111.5 & 382. & 1089. & $<0.030^{5}$ \\
\hline J1028-5819 & $\mathrm{r}$ & 285.06 & -0.50 & 91.4 & 16.1 & 83.3 & 0.36 \\
\hline J1044-5737 & gu & 286.57 & 1.16 & 139.0 & 54.6 & 80.2 & $<0.020^{5}$ \\
\hline J1048-5832 & $\mathrm{r}$ & 287.42 & 0.58 & 123.7 & 95.7 & 200 & 6.5 \\
\hline J1057-5226 & re & 285.98 & 6.65 & 197.1 & 5.8 & 3.0 & 9.5 \\
\hline J1105-6107 & $\mathrm{r}$ & 290.49 & -0.85 & 63.2 & 15.8 & 248. & 0.75 \\
\hline $\mathrm{J} 1112-6103$ & $\mathrm{r}$ & 291.22 & -0.46 & 65.0 & 31.5 & 454. & 1.4 \\
\hline J1119-6127 & $\mathrm{r}$ & 292.15 & -0.54 & 408.7 & 4028. & 233. & 0.8 \\
\hline J1124-5916 & $\mathrm{r}$ & 292.04 & 1.75 & 135.5 & 750. & 1190. & 0.08 \\
\hline J1135-6055 & $\mathrm{gu}$ & 293.79 & 0.58 & 114.5 & 78.4 & 206. & $<0.030^{4}$ \\
\hline $\mathrm{J} 1357-6429$ & $\mathrm{r}$ & 309.92 & -2.51 & 166.2 & 357. & 307. & 0.44 \\
\hline J1410-6132 & $\mathrm{r}$ & 312.20 & -0.09 & 50.1 & 31.8 & 1000. & $6.56^{6}$ \\
\hline $\mathrm{J} 1413-6205$ & $\mathrm{gu}$ & 312.37 & -0.74 & 109.7 & 27.4 & 81.8 & $<0.024^{5}$ \\
\hline $\mathrm{J} 1418-6058$ & $\mathrm{gu}$ & 313.32 & 0.13 & 110.6 & 169. & 494. & $<0.029^{1}$ \\
\hline $\mathrm{J} 1420-6048$ & $\mathrm{r}$ & 313.54 & 0.23 & 68.2 & 82.9 & 1032. & 0.9 \\
\hline J1429-5911 & $\mathrm{gu}$ & 315.26 & 1.30 & 115.8 & 30.5 & 77.4 & $<0.021^{5}$ \\
\hline J1459-6053 & gu & 317.89 & -1.79 & 103.2 & 25.3 & 90.9 & $<0.037^{1}$ \\
\hline $\mathrm{J} 1509-5850$ & $\mathrm{r}$ & 319.97 & -0.62 & 88.9 & 9.2 & 51.5 & 0.15 \\
\hline J1513-5908 & xe & 320.32 & -1.16 & 151.5 & 1529. & 1735. & 0.94 \\
\hline $\mathrm{J} 1531-5610$ & $\mathrm{r}$ & 323.90 & 0.03 & 84.2 & 13.8 & 91.2 & 0.6 \\
\hline J1620-4927 & gu & 333.89 & 0.41 & 171.9 & 10.5 & 8.1 & $<0.040^{2}$ \\
\hline J1648-4611 & $\mathrm{r}$ & 339.44 & -0.79 & 165.0 & 23.7 & 20.9 & 0.58 \\
\hline $\mathrm{J} 1702-4128$ & $\mathrm{r}$ & 344.74 & 0.12 & 182.2 & 52.3 & 34.2 & 1.1 \\
\hline J1709-4429 & re & 343.10 & -2.69 & 102.5 & 92.8 & 340. & 7.3 \\
\hline J1718-3825 & $\mathrm{r}$ & 348.95 & -0.43 & 74.7 & 13.2 & 125. & 1.3 \\
\hline $\mathrm{J} 1730-3350$ & $\mathrm{r}$ & 354.13 & 0.09 & 139.5 & 84.8 & 123. & 3.2 \\
\hline $\mathrm{J} 1732-3131$ & gu & 356.31 & 1.01 & 196.5 & 28.0 & 14.6 & $<0.015^{1}$ \\
\hline J1741-2054 & gu & 6.43 & 4.91 & 413.7 & 17.0 & 0.9 & 0.16 \\
\hline J1746-3239 & $\mathrm{gu}$ & 356.96 & -2.18 & 199.5 & 6.6 & 3.3 & $<0.034^{2}$ \\
\hline $\mathrm{J} 1747-2958$ & $\mathrm{r}$ & 359.31 & -0.84 & 98.8 & 61.3 & 251. & 0.25 \\
\hline $\mathrm{J} 1801-2451$ & $\mathrm{r}$ & 5.25 & -0.88 & 125.0 & 127. & 257. & 0.85 \\
\hline $\mathrm{J} 1803-2149$ & gu & 8.14 & 0.19 & 106.3 & 19.5 & 64.1 & $<0.024^{2}$ \\
\hline $\mathrm{J} 1809-2332$ & $\mathrm{~g}$ & 7.39 & -1.99 & 146.8 & 34.4 & 43.0 & $<0.025^{1}$ \\
\hline $\mathrm{J} 1813-1246$ & $\mathrm{gu}$ & 17.24 & 2.44 & 48.1 & 17.6 & 624. & $<0.017^{1}$ \\
\hline $\mathrm{J} 1826-1256$ & $\mathrm{~g}$ & 18.56 & -0.38 & 110.2 & 121. & 358. & $<0.013^{1}$ \\
\hline $\mathrm{J} 1833-1034$ & $\mathrm{r}$ & 21.50 & -0.89 & 61.9 & 202. & 3364. & 0.071 \\
\hline $\mathrm{J} 1835-1106$ & $\mathrm{r}$ & 21.22 & -1.51 & 165.9 & 20.6 & 17.8 & 2.2 \\
\hline $\mathrm{J} 1836+5925$ & $\mathrm{~g}$ & 88.88 & 25.00 & 173.3 & 1.5 & 1.1 & $<0.004^{1}$ \\
\hline J1838-0537 & $\mathrm{gu}$ & 26.51 & 0.21 & 145.7 & 465. & 593. & $<0.017^{7}$ \\
\hline $\mathrm{J} 1846+0919$ & gu & 40.69 & 5.34 & 225.6 & 9.9 & 3.4 & $<0.005^{5}$ \\
\hline $\mathrm{J} 1907+0602$ & $\mathrm{~g}$ & 40.18 & -0.89 & 106.6 & 86.7 & 282. & 0.0034 \\
\hline $\mathrm{J} 1952+3252$ & re & 68.77 & 2.82 & 39.5 & 5.8 & 372. & 1.0 \\
\hline $\mathrm{J} 1954+2836$ & $\mathrm{gu}$ & 65.24 & 0.38 & 92.7 & 21.2 & 105. & $<0.005^{5}$ \\
\hline $\mathrm{J} 1957+5033$ & $\mathrm{gu}$ & 84.60 & 11.00 & 374.8 & 6.8 & 0.5 & $<0.010^{5}$ \\
\hline $\mathrm{J} 1958+2846$ & $\mathrm{gu}$ & 65.88 & -0.35 & 290.4 & 212 & 34.2 & $<0.006^{1}$ \\
\hline
\end{tabular}


Table 2

(Continued)

\begin{tabular}{lccccccc}
\hline \hline PSR & History & $\begin{array}{c}l \\
\left({ }^{\circ}\right)\end{array}$ & $\begin{array}{c}b \\
\left({ }^{\circ}\right)\end{array}$ & $\begin{array}{c}P \\
(\mathrm{~ms})\end{array}$ & $\begin{array}{c}\dot{P} \\
\left(10^{-15}\right)\end{array}$ & $\begin{array}{c}\dot{E} \\
\left(10^{34} \mathrm{erg} \mathrm{s}^{-1}\right)\end{array}$ & $\begin{array}{c}S_{1400} \\
(\mathrm{mJy})\end{array}$ \\
\hline $\mathrm{J} 2021+3651$ & $\mathrm{r}$ & 75.22 & 0.11 & 103.7 & 95.6 & 338. & 0.1 \\
$\mathrm{~J} 2021+4026$ & g & 78.23 & 2.09 & 265.3 & 54.2 & 11.4 & $<0.020^{1}$ \\
$\mathrm{~J} 2028+3332$ & gu & 73.36 & -3.01 & 176.7 & 4.9 & 3.5 & $<0.005^{2}$ \\
$\mathrm{~J} 2030+3641$ & ru & 76.12 & -1.44 & 200.1 & 6.5 & 3.2 & 0.15 \\
$\mathrm{~J} 2030+4415$ & gu & 82.34 & 2.89 & 227.1 & 6.5 & 2.2 & $<0.008^{2}$ \\
$\mathrm{~J} 2032+4127$ & gu & 80.22 & 1.03 & 143.2 & 20.4 & 27.3 & 0.23 \\
$\mathrm{~J} 2043+2740$ & $\mathrm{r}$ & 70.61 & -9.15 & 96.1 & 1.2 & 5.5 & 9.35 \\
$\mathrm{~J} 2055+2539$ & gu & 70.69 & -12.52 & 319.6 & 4.1 & 0.5 & $<0.007^{5}$ \\
$\mathrm{~J} 2111+4606$ & gu & 88.31 & -1.45 & 157.8 & 143. & 144. & $<0.013^{2}$ \\
$\mathrm{~J} 2139+4716$ & gu & 92.63 & -4.02 & 282.8 & 1.8 & 0.3 & $<0.014^{2}$ \\
$\mathrm{~J} 2229+6114$ & $\mathrm{r}$ & 106.65 & 2.95 & 51.6 & 77.9 & 2231. & 0.25 \\
$\mathrm{~J} 2238+5903$ & gu & 106.56 & 0.48 & 162.7 & 97.0 & 88.8 & $<0.011^{1}$ \\
$\mathrm{~J} 2240+5832$ & $\mathrm{r}$ & 106.57 & -0.11 & 139.9 & 15.2 & 21.9 & 2.7 \\
\hline
\end{tabular}

Notes. Column 2 gives a discovery/detection code: $\mathrm{g}=$ gamma-ray blind search, $\mathrm{r}=$ radio, $\mathrm{u}=$ candidate location was that of an unassociated LAT source, $\mathrm{x}=\mathrm{X}$-ray, $\mathrm{e}=$ seen by EGRET. Columns 3 and 4 give Galactic coordinates for each pulsar. Columns 5 and 6 list the period $(P)$ and its first derivative $(\dot{P})$, and Column 7 gives the spindown luminosity $\dot{E}$. The Shklovskii correction to $\dot{P}$ and $\dot{E}$ is negligible for these young pulsars (see Section 4.3). Column 8 gives the radio flux density (or upper limit) at $1400 \mathrm{MHz}\left(S_{1400}\right.$; see Section 4.1), taken from the ATNF database except for the noted entries where: (1) Ray et al. (2011); (2) Pletsch et al. (2012a); (3) Geminga: Spoelstra \& Hermsen (1984); (4) GBT (this paper); (5) Saz Parkinson et al. (2010); (6) O'Brien et al. (2008); (7) Pletsch et al. (2012b). PSR J1509-5850 should not be confused with PSR B1509-58 (= J1513-5908) observed by instruments on the Compton Gamma-Ray Observatory.

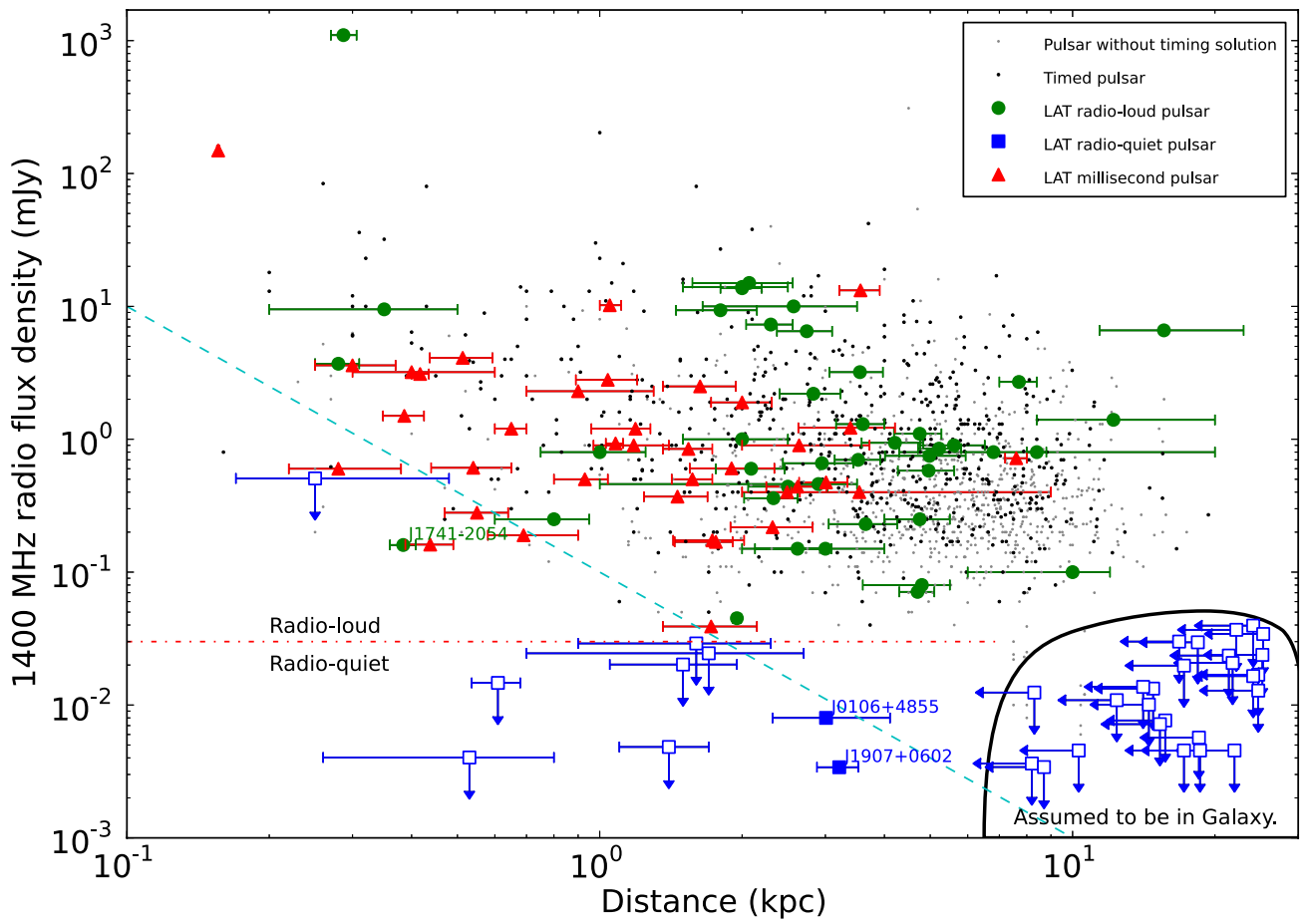

Figure 3. Radio flux density at $1400 \mathrm{MHz}$ vs. pulsar distance. Markers are as in Figure 1, except that blue open squares show pulsars discovered in gamma-ray blind period searches for which no radio signal has been detected. The horizontal line at $30 \mu \mathrm{Jy}$ is our convention for distinguishing radio "loud" from radio "quiet" pulsars. The diagonal line shows a threshold in pseudo-luminosity of $100 \mu \mathrm{Jy}-\mathrm{kpc}^{2}$. Four gamma-discovered pulsars have been detected at radio frequencies: two are radio-quiet and are labeled. Of the two that are radio loud, one is labeled while PSR J2032+4127 is in the cloud of points. The pulsars at lower-right are assigned distance limits along the Milky Way's rim in Figure 4.

(A color version of this figure is available in the online journal.)

use can be subjective. Tables 5 and 6 list the distance estimates that we adopt, the methods with which these estimates were acquired, and the appropriate references.

The most accurate distance estimator is the annual trigonometric parallax. Unfortunately, parallax can only be measured for relatively nearby pulsars, using X-ray or optical images, ra- dio interferometric imaging, or accurate timing. For 14 Fermi pulsars a parallax has been measured. We rejected two with low-significance $(<2 \sigma)$. For the remaining 12 pulsars we consider this the best distance estimate. One caveat when converting parallax measurements to distances is the Lutz-Kelker effect, an overestimate of parallax values (and hence underestimate of 
Table 3

Some Parameters of LAT-detected Millisecond Pulsars

\begin{tabular}{|c|c|c|c|c|c|c|c|}
\hline PSR & $\begin{array}{l}\text { Type, } \\
\text { History }\end{array}$ & $\begin{array}{c}l \\
\left({ }^{\circ}\right)\end{array}$ & $\begin{array}{l}b \\
\left({ }^{\circ}\right)\end{array}$ & $\begin{array}{c}P \\
(\mathrm{~ms})\end{array}$ & $\begin{array}{c}\dot{P} \\
\left(10^{-20}\right)\end{array}$ & $\begin{array}{c}\dot{E} \\
\left(10^{-33} \mathrm{erg} \mathrm{s}^{-1}\right)\end{array}$ & $\begin{array}{l}S_{1400} \\
(\mathrm{mJy})\end{array}$ \\
\hline $\mathrm{J} 0023+0923$ & bwru & 111.15 & -53.22 & 3.05 & 1.08 & 15.1 & $\overline{0.19^{1}}$ \\
\hline J0030+0451 & $\mathrm{r}$ & 113.14 & -57.61 & 4.87 & 1.02 & 3.49 & 0.60 \\
\hline J0034-0534 & br & 111.49 & -68.07 & 1.88 & 0.50 & 29.7 & 0.61 \\
\hline J0101-6422 & bru & 301.19 & -52.72 & 2.57 & 0.48 & 12.0 & 0.28 \\
\hline J0102+4839 & bru & 124.93 & -14.83 & 2.96 & 1.17 & 17.5 & $0.22^{2}$ \\
\hline J0218+4232 & br & 139.51 & -17.53 & 2.32 & 7.74 & 243. & 0.90 \\
\hline $\mathrm{J} 0340+4130$ & $\mathrm{ru}$ & 154.04 & -11.47 & 3.30 & 0.59 & 7.9 & $0.17^{2}$ \\
\hline J0437-4715 & br & 253.39 & -41.96 & 5.76 & 5.73 & 11.8 & 149 \\
\hline J0610-2100 & bwr & 227.75 & -18.18 & 3.86 & 1.23 & 8.5 & 0.40 \\
\hline J0613-0200 & br & 210.41 & -9.30 & 3.06 & 0.96 & 13.2 & 2.3 \\
\hline J0614-3329 & bru & 240.50 & -21.83 & 3.15 & 1.78 & 22.0 & $0.60^{3}$ \\
\hline J0751+1807 & br & 202.73 & 21.09 & 3.48 & 0.78 & 7.30 & 3.2 \\
\hline J1024-0719 & $\mathrm{r}$ & 251.70 & 40.52 & 5.16 & 1.85 & 5.30 & 1.5 \\
\hline $\mathrm{J} 1124-3653$ & bwru & 283.74 & 23.59 & 2.41 & 0.58 & 17.1 & $0.04^{4}$ \\
\hline J1125-5825 & br & 291.89 & 2.60 & 3.10 & 6.09 & 80.5 & 0.44 \\
\hline $\mathrm{J} 1231-1411$ & bru & 295.53 & 48.39 & 3.68 & 2.12 & 17.9 & $0.16^{3}$ \\
\hline J1446-4701 & br & 322.50 & 11.43 & 2.19 & 0.98 & 36.8 & 0.37 \\
\hline J1514-4946 & bru & 325.22 & 6.84 & 3.58 & 1.87 & 16.0 & $\ldots$ \\
\hline $\mathrm{J} 1600-3053$ & br & 344.09 & 16.45 & 3.60 & 0.95 & 8.05 & 2.5 \\
\hline $\mathrm{J} 1614-2230$ & br & 352.64 & 20.19 & 3.15 & 0.96 & 12.1 & $1.2^{5}$ \\
\hline J1658-5324 & $\mathrm{ru}$ & 334.87 & -6.63 & 2.43 & 1.10 & 30.2 & $0.50^{6}$ \\
\hline $\mathrm{J} 1713+0747$ & br & 28.75 & 25.22 & 4.57 & 0.85 & 3.53 & 10.2 \\
\hline $\mathrm{J} 1741+1351$ & br & 37.90 & 21.62 & 3.75 & 3.02 & 22.7 & 0.93 \\
\hline J1744-1134 & $\mathrm{r}$ & 14.79 & 9.18 & 4.07 & 0.89 & 5.20 & 3.1 \\
\hline $\mathrm{J} 1747-4036$ & $\mathrm{ru}$ & 350.19 & -6.35 & 1.64 & 1.33 & 116. & $1.22^{6}$ \\
\hline $\mathrm{J} 1810+1744$ & bwru & 43.87 & 16.64 & 1.66 & 0.46 & 39.7 & $1.89^{1}$ \\
\hline $\mathrm{J} 1823-3021 \mathrm{~A}$ & $\mathrm{r}$ & 2.79 & -7.91 & 5.44 & 338. & 828. & 0.72 \\
\hline J1858-2216 & bru & 13.55 & -11.45 & 2.38 & 0.39 & 11.3 & $\ldots$ \\
\hline J1902-5105 & bru & 345.59 & -22.40 & 1.74 & 0.90 & 68.6 & $0.90^{6}$ \\
\hline J1939+2134 & $\mathrm{r}$ & 57.51 & -0.29 & 1.56 & 10.5 & 1097. & 13.9 \\
\hline $\mathrm{J} 1959+2048$ & bwr & 59.20 & -4.70 & 1.61 & 1.68 & 160 & 0.40 \\
\hline $\mathrm{J} 2017+0603$ & bru & 48.62 & -16.03 & 2.90 & 0.83 & 13.0 & 0.50 \\
\hline $\mathrm{J} 2043+1711$ & bru & 61.92 & -15.31 & 2.38 & 0.57 & 15.3 & $0.17^{7}$ \\
\hline $\mathrm{J} 2047+1053$ & bru & 57.06 & -19.67 & 4.29 & 2.10 & 10.5 & $\ldots$ \\
\hline J2051-0827 & bwr & 39.19 & -30.41 & 4.51 & 1.28 & 5.49 & 2.8 \\
\hline $\mathrm{J} 2124-3358$ & $\mathrm{r}$ & 10.93 & -45.44 & 4.93 & 2.06 & 6.77 & 3.6 \\
\hline $\mathrm{J} 2214+3000$ & bwru & 86.86 & -21.67 & 3.12 & 1.50 & 19.2 & $0.85^{3}$ \\
\hline $\mathrm{J} 2215+5135$ & bkru & 99.46 & -4.60 & 2.61 & 2.34 & 51.9 & $0.47^{1}$ \\
\hline J2241-5236 & bwru & 337.46 & -54.93 & 2.19 & 0.87 & 26.0 & 4.1 \\
\hline $\mathrm{J} 2302+4442$ & bru & 103.40 & -14.00 & 5.20 & 1.33 & 3.82 & 1.2 \\
\hline
\end{tabular}

Notes. Column 2: $\mathrm{b}=$ binary, $\mathrm{r}=$ radio-detected, $\mathrm{u}=$ seed position was that of an unassociated LAT source, $\mathrm{w}=$ white dwarf companion, $\mathrm{k}=$ "redback." Columns 3 and 4 give the Galactic coordinates, with the rotation period $P$ in column 5. The first period time derivative $\dot{P}$ and the spindown luminosity $\dot{E}$ in Columns 6 and 7 are uncorrected for the Shklovskii effect in this table. The corrected values are used throughout the rest of the paper, and are listed in Table 6. Column 9 gives the radio flux density (or upper limit) at $1400 \mathrm{MHz}$ (Section 4.1), taken from the ATNF database except for the noted entries: (1) Hessels et al. (2011); (2) P. Bangale et al. (in preparation); (3) Ransom et al. (2011); (4) This paper; (5) Demorest et al. (2010); (6) Kerr et al. (2012); (7) Guillemot et al. (2012). The three MSPs with no $S_{1400}$ listed scintillate too much to obtain a good flux measurement (PSR J1514-4946), or the radio flux has not yet been measured (PSRs J1858-2216 and J2047+1053).

distances) that must be corrected for the larger volume of space traced by smaller parallax values (Lutz \& Kelker 1973). We use the Lutz-Kelker corrected distance estimates determined by Verbiest et al. (2012).

The dispersion measure (DM) is by far the most commonly used pulsar distance estimator. DM is the column density of free electrons along the path from Earth to the pulsar, in units of $\mathrm{pc} \mathrm{cm}^{-3}$. The electrons delay the radio pulse arrival by $\Delta t=\operatorname{DM}\left(p v^{2}\right)^{-1}$ where $v$ is the observation frequency in $\mathrm{MHz}$ and $p=2.410 \times 10^{-4} \mathrm{MHz}^{-2} \mathrm{pc} \mathrm{cm}^{-3} \mathrm{~s}^{-1}$. Given a model for the electron density $n_{e}$ in the various structures of our Galaxy, integrating $\mathrm{DM}=\int_{0}^{d} n_{e} d l$ along the line of sight $d l$ yields the distance $d$ for which DM matches the radio measurement. In this work we use the NE2001 model (Cordes \& Lazio 2002), available as off-line code. To estimate the distance errors we re-run NE2001 twice, using DM $\pm 20 \%$, as the authors recommend. The measured DM uncertainty is much lower than this, but this accommodates unmodeled electron-rich or poor regions. This yields distance uncertainties less than $30 \%$ for many pulsars. Nevertheless, significant discrepancies with the true pulsar distances along some lines of sight still occur. As examples, the DM distances for PSR J2021+3651 (Abdo et al. 2009d) and PSR J0248+6021 (Theureau et al. 2011) may be more than three times greater than the true distances. 


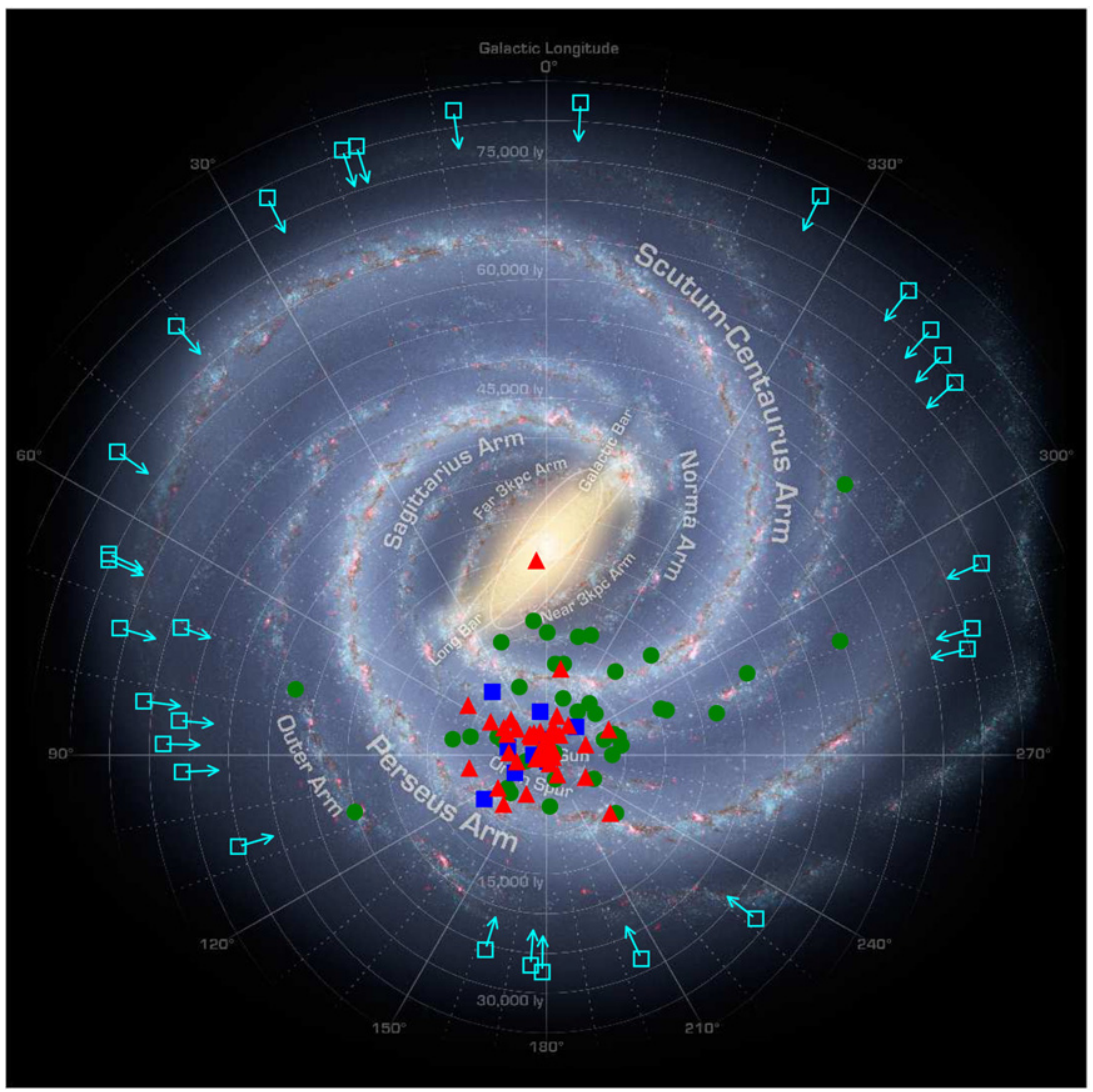

Figure 4. Gamma-ray pulsar positions projected onto the Milky Way model of Reid et al. (2009). The pulsar that appears to be coincident with the Galactic center, PSR J1823-3021A in the globular cluster NGC 6624, lies well above the Galactic plane. Distance uncertainties are not shown for clarity, however, they can be quite large, especially for the more distant objects. The open squares with arrows indicate the lines of sight toward pulsars for which no distance estimates exist, placed at the distances where $95 \%$ of the electron column density has been integrated in the NE2001 model. The markers are the same as in Figure 1.

(A color version of this figure is available in the online journal.)

Table 4

Gamma-Ray Pulsars Not in This Catalog

\begin{tabular}{|c|c|c|c|c|}
\hline PSRJ & $\begin{array}{c}P \\
(\mathrm{~ms})\end{array}$ & $\begin{array}{c}\dot{E} \\
\left(10^{34} \mathrm{erg} \mathrm{s}^{-1}\right)\end{array}$ & Codes & References \\
\hline $\mathrm{J} 0307+7443$ & 3.16 & 2.2 & $\mathrm{mbr}$ & Ray et al. (2012) \\
\hline J0737-3039A & 22.7 & 0.59 & $\mathrm{r}$ & Guillemot et al. (2013) \\
\hline $\mathrm{J} 1055-6028$ & 99.7 & 120 & r & Hou \& Smith (2013) \\
\hline $\mathrm{J} 1311-3430$ & 2.56 & 4.9 & mbgu & Pletsch et al. (2012c) \\
\hline J1544+4937 & 2.16 & 1.2 & mbru & Bhattacharyya et al. (2013) \\
\hline $\mathrm{J} 1640+2224$ & 3.16 & 0.35 & $\mathrm{mbr}$ & Hou \& Smith (2013) \\
\hline J1705-1906 & 299.0 & 0.61 & $\mathrm{r}$ & Hou \& Smith (2013) \\
\hline J1732-5049 & 5.31 & 0.37 & $\mathrm{mrb}$ & Hou \& Smith (2013) \\
\hline $\mathrm{J} 1745+1017$ & 2.65 & 0.53 & mbru & Barr et al. (2013) \\
\hline $\mathrm{J} 1816+4510$ & 3.19 & 5.2 & mbru & Kaplan et al. (2012) \\
\hline $\mathrm{J} 1824-2452 \mathrm{~A}$ & 3.05 & 220 & $\mathrm{mr}$ & Wu et al. (2013); T. J. Johnson et al. (in preparation) \\
\hline $\mathrm{J} 1843-1113$ & 1.85 & 6.0 & $\mathrm{mr}$ & Hou \& Smith (2013) \\
\hline $\mathrm{J} 1913+0904$ & 163.2 & 16 & $\mathrm{r}$ & Hou \& Smith (2013) \\
\hline J2256-1024 & 2.29 & 5.2 & mbru & Boyles et al. (2011) \\
\hline J2339-0533 & 2.88 & 2.3 & mbru & P. S. Ray et al. (in preparation) \\
\hline
\end{tabular}

Notes. Beyond the 117 pulsars. The above 15 pulsars were discovered in gamma rays as this catalog neared completion. An additional 13 gamma-ray pulsars discovered by the LAT collaboration or by other groups using public LAT data have publications in preparation, for a total of 145 as we go to submission (2013 May 8). We maintain a list at https://confluence.slac.stanford.edu/ display/GLAMCOG/Public+List+of+LAT-Detected+Gamma-Ray+Pulsars. The codes are: $\mathrm{u}=$ discovered in a LAT unassociated source, $\mathrm{g}=$ discovered in a gamma-ray blind period search, $\mathrm{r}=$ radio detection, $\mathrm{m}=\mathrm{MSP}, \mathrm{b}=$ binary system.

For some pulsars, an absorbing hydrogen column density $N_{\mathrm{H}}$ below $1 \mathrm{keV}$ has been obtained (see Section 9.1). Comparing $N_{\mathrm{H}}$ with the total hydrogen column density for that line of sight obtained from $21 \mathrm{~cm}$ radio surveys yields a rough distance es- timate. The Doppler shift of neutral hydrogen (HI) absorption or emission lines measured from clouds on the line of sight, together with a Galactic rotation model as described in Section 4.3, can give "kinematic" distances to the clouds. The pulsar distance 
Table 5

Distance Estimates for Young LAT-detected Pulsars

\begin{tabular}{|c|c|c|c|}
\hline Pulsar Name & $\begin{array}{l}\text { Distance } \\
(\mathrm{kpc})\end{array}$ & Method & Reference \\
\hline $\mathrm{J} 0007+7303$ & $1.4 \pm 0.3$ & $\mathrm{~K}$ & Pineault et al. (1993) \\
\hline $\mathrm{J} 0106+4855$ & $3.0_{-0.7}^{+1.1}$ & $\mathrm{DM}$ & Pletsch et al. (2012a) \\
\hline J0205+6449 & $1.95 \pm 0.04$ & KP & Xu et al. (2006) \\
\hline J0248+6021 & $2.0 \pm 0.2$ & $\mathrm{~K}$ & Theureau et al. (2011) \\
\hline J0357+3205 & $<8.2$ & DMM & $\ldots$ \\
\hline J0534+2200 & $2.0 \pm 0.5$ & $\mathrm{O}$ & Trimble (1973) \\
\hline J0622+3749 & $<8.3$ & DMM & $\ldots$ \\
\hline J0631+1036 & $1.0 \pm 0.2$ & $\mathrm{O}$ & Zepka et al. (1996) \\
\hline $\mathrm{J} 0633+0632$ & $<8.7$ & DMM & $\ldots$ \\
\hline J0633+1746 & $0.25_{-0.08}^{+0.23}$ & $\mathrm{P}$ & Verbiest et al. (2012) \\
\hline J0659+1414 & $0.28 \pm 0.03$ & $\mathrm{P}$ & Verbiest et al. (2012) \\
\hline J0729-1448 & $3.5 \pm 0.4$ & $\mathrm{DM}$ & Morris et al. (2002) \\
\hline J0734-1559 & $<10.3$ & DMM & $\ldots$ \\
\hline J0742-2822 & $2.1 \pm 0.5$ & DM & Janssen \& Stappers (2006) \\
\hline J0835-4510 & $0.29_{-0.02}^{+0.02}$ & $\mathrm{P}$ & Dodson et al. (2003) \\
\hline J0908-4913 & $2.6 \pm 0.9$ & $\mathrm{DM}$ & Hobbs et al. (2004a) \\
\hline J0940-5428 & $3.0 \pm 0.5$ & $\mathrm{DM}$ & Manchester et al. (2001) \\
\hline J1016-5857 & $2.9_{-1.9}^{+0.6}$ & $\mathrm{~K}$ & Ruiz \& May (1986) \\
\hline J1019-5749 & $6.8_{-2.5}^{+13.2}$ & $\mathrm{DM}$ & Kramer et al. (2003) \\
\hline J1023-5746 & $<16.8$ & DMM & $\ldots$ \\
\hline J1028-5819 & $2.3 \pm 0.3$ & DM & Keith et al. (2008) \\
\hline J1044-5737 & $<17.2$ & DMM & $\ldots$ \\
\hline J1048-5832 & $2.7 \pm 0.4$ & DM & Johnston et al. (1995) \\
\hline J1057-5226 & $0.3 \pm 0.2$ & $\mathrm{O}$ & Mignani et al. (2010b) \\
\hline J1105-6107 & $5.0 \pm 1.0$ & $\mathrm{DM}$ & Kaspi et al. (1997) \\
\hline J1112-6103 & $12.2_{-3.8}^{+7.8}$ & $\mathrm{DM}$ & Manchester et al. (2001) \\
\hline J1119-6127 & $8.4 \pm 0.4$ & $\mathrm{~K}$ & Caswell et al. (2004) \\
\hline J1124-5916 & $4.8_{-1.2}^{+0.7}$ & $\mathrm{X}$ & Gonzalez \& Safi-Harb (2003) \\
\hline J1135-6055 & $<18.4$ & DMM & $\ldots$ \\
\hline J1357-6429 & $2.5_{-0.4}^{+0.5}$ & $\mathrm{DM}$ & Lorimer et al. (2006) \\
\hline J1410-6132 & $15.6_{-4.2}^{+7.4}$ & $\mathrm{DM}$ & O'Brien et al. (2008) \\
\hline J1413-6205 & $<21.4$ & DMM & $\ldots$ \\
\hline J1418-6058 & $1.6 \pm 0.7$ & $\mathrm{O}$ & Yadigaroglu \& Romani (1997) \\
\hline J1420-6048 & $5.6 \pm 0.9$ & DM & Weltevrede et al. (2010) \\
\hline J1429-5911 & $<21.8$ & DMM & $\ldots$ \\
\hline $\mathrm{J} 1459-6053$ & $<22.2$ & DMM & $\ldots$ \\
\hline $\mathrm{J} 1509-5850$ & $2.6 \pm 0.5$ & DM & Weltevrede et al. (2010) \\
\hline J1513-5908 & $4.2 \pm 0.6$ & $\mathrm{DM}$ & Hobbs et al. (2004a) \\
\hline $\mathrm{J} 1531-5610$ & $2.1_{-0.3}^{+0.4}$ & $\mathrm{DM}$ & Kramer et al. (2003) \\
\hline J1620-4927 & $<24.1$ & DMM & $\ldots$ \\
\hline J1648-4611 & $5.0 \pm 0.7$ & DM & Kramer et al. (2003) \\
\hline J1702-4128 & $4.8 \pm 0.6$ & $\mathrm{DM}$ & Kramer et al. (2003) \\
\hline J1709-4429 & $2.3 \pm 0.3$ & $\mathrm{DM}$ & Johnston et al. (1995) \\
\hline J1718-3825 & $3.6 \pm 0.4$ & $\mathrm{DM}$ & Manchester et al. (2001) \\
\hline J1730-3350 & $3.5_{-0.5}^{+0.4}$ & $\mathrm{DM}$ & Hobbs et al. (2004b) \\
\hline $\mathrm{J} 1732-3131$ & $0.6 \pm 0.1$ & $\mathrm{DM}$ & Maan et al. (2012) \\
\hline J1741-2054 & $0.38 \pm 0.02$ & DM & Camilo et al. (2009) \\
\hline J1746-3239 & $<25.3$ & DMM & $\ldots$ \\
\hline $\mathrm{J} 1747-2958$ & $4.8 \pm 0.8$ & $\mathrm{X}$ & Gaensler et al. (2004) \\
\hline J1801-2451 & $5.2_{-0.5}^{+0.6}$ & $\mathrm{DM}$ & Hobbs et al. (2004b) \\
\hline J1803-2149 & $<25.2$ & DMM & $\ldots$ \\
\hline $\mathrm{J} 1809-2332$ & $1.7 \pm 1.0$ & K & Oka et al. (1999) \\
\hline J1813-1246 & $<24.7$ & DMM & $\ldots$ \\
\hline J1826-1256 & $<24.7$ & DMM & $\ldots$ \\
\hline J1833-1034 & $4.7 \pm 0.4$ & $\mathrm{~K}$ & Gupta et al. (2005); Camilo et al. (2006) \\
\hline J1835-1106 & $2.8 \pm 0.4$ & $\mathrm{DM}$ & D’Amico et al. (1998) \\
\hline $\mathrm{J} 1836+5925$ & $0.5 \pm 0.3$ & $\mathrm{X}$ & Halpern et al. (2002) \\
\hline J1838-0537 & $<24.1$ & DMM & $\ldots$ \\
\hline J1846+0919 & $<22.0$ & DMM & $\ldots$ \\
\hline $\mathrm{J} 1907+0602$ & $3.2 \pm 0.3$ & DM & Abdo et al. (20101) \\
\hline $\mathrm{J} 1952+3252$ & $2.0 \pm 0.5$ & $\mathrm{~K}$ & Greidanus \& Strom (1990) \\
\hline $\mathrm{J} 1954+2836$ & $<18.6$ & DMM & $\ldots$ \\
\hline \multirow[t]{2}{*}{$\mathrm{J} 1957+5033$} & $<14.5$ & DMM & $\ldots$ \\
\hline & & 12 & \\
\hline
\end{tabular}


Table 5

(Continued)

\begin{tabular}{lccc}
\hline \hline Pulsar Name & $\begin{array}{c}\text { Distance } \\
(\mathrm{kpc})\end{array}$ & Method & Reference \\
\hline $\mathrm{J} 1958+2846$ & $<18.5$ & DMM & \\
$\mathrm{J} 2021+3651$ & $10.0_{-4.0}^{+2.0}$ & $\mathrm{O}$ & $\ldots$ \\
$\mathrm{J} 2021+4026$ & $1.5 \pm 0.4$ & $\mathrm{~K}$ & Hessels et al. (2004) \\
$\mathrm{J} 2028+3332$ & $<17.2$ & DMM & Landecker et al. (1980) \\
$\mathrm{J} 2030+3641$ & $3.0 \pm 1.0$ & $\mathrm{O}$ & $\ldots$ \\
$\mathrm{J} 2030+4415$ & $<15.7$ & DMM & Camilo et al. (2012) \\
$\mathrm{J} 2032+4127$ & $3.7 \pm 0.6$ & DM & $\ldots$ \\
$\mathrm{J} 2043+2740$ & $1.8 \pm 0.3$ & $\mathrm{DM}$ & Camilo et al. (2009) \\
$\mathrm{J} 2055+2539$ & $<15.3$ & DMM & Ray et al. (1996) \\
$\mathrm{J} 2111+4606$ & $<14.8$ & DMM & $\ldots$ \\
$\mathrm{J} 2139+4716$ & $<14.1$ & DMM & $\ldots$ \\
$\mathrm{J} 2229+6114$ & $0.80_{-0.20}^{+0.15}$ & $\mathrm{~K}$ & $\ldots$ \\
$\mathrm{J} 2238+5903$ & $<12.4$ & DMM & Kothes et al. (2001) \\
$\mathrm{J} 2240+5832$ & $7.7 \pm 0.7$ & $\mathrm{O}$ & $\ldots$ \\
\end{tabular}

Notes. The best known distances of the 77 young pulsars detected by Fermi. The methods are: K-kinematic method; P-parallax; DM — from dispersion measure using the Cordes \& Lazio (2002) NE2001 model; X-from $\mathrm{X}$-ray measurements; O-other methods. For DM, the reference gives the DM measurement. For the 26 pulsars with no distance estimate, DMM is the distance to the Galaxy's edge, taken as an upper limit, determined from the maximum NE2001 DM value for that line of sight.

is then constrained if there is evidence that the pulsar is in one of the clouds, or between some of them. Associations can be uncertain and these distance estimates can be controversial.

With the growing number of gamma-ray pulsars not detected at radio wavelengths, and thus without a DM, and the difficulties of the other methods, we face an ever-growing pulsar distance problem. We have 26 objects with no distance estimates, compared to nine in 1PC. To mitigate this, we determine a maximum distance by assuming that the pulsar is within the Galaxy. We define the Galaxy edge as the distance for a given line of sight where the NE2001 DM reaches its maximum value ("DMM" in Table 5; illustrated in Figure 4).

\subsection{Doppler Corrections}

Many pulsar characteristics, including some listed in Tables 2 and 3 , depend on the intrinsic spin period $P^{\text {int }}$ and spindown rate $\dot{P}^{\text {int }}$. The Doppler shift of the observed period is $P=$ $\left(1+v_{\mathrm{R}} / c\right) P^{\text {int }}$, where $v_{\mathrm{R}}$ is the pulsar's radial velocity along the unit vector $\mathbf{n}_{10}$ from the solar system. The Doppler correction to $\dot{P}$ is obtained by differentiating the equation and separating the effects of the system's proper motion (Shklovskii 1970) from the acceleration due to Galactic rotation:

$$
\dot{P}^{\text {int }}=\dot{P}-\dot{P}^{\text {shk }}-\dot{P}^{\text {gal }}
$$

with

$$
\dot{P}^{\text {shk }}=\frac{1}{c} \mu^{2} d P=k\left(\frac{\mu}{\text { mas yr }^{-1}}\right)^{2}\left(\frac{d}{\mathrm{kpc}}\right)\left(\frac{P}{\mathrm{~s}}\right)
$$

and

$$
\dot{P}^{\text {gal }}=\frac{1}{c} \mathbf{n}_{10} \cdot\left(\mathbf{a}_{1}-\mathbf{a}_{0}\right) P
$$

where $k=2.43 \times 10^{-21}$ for pulsar distance $d$ and proper motion transverse to the line of sight $\mu$. The Galactic potential model of Carlberg \& Innanen (1987) and Kuijken \& Gilmore (1989) provides the accelerations $\mathbf{a}_{1}$ of the pulsar and $\mathbf{a}_{0}$ of the Sun. Since the constant $k$ is small, the corrections are negligible for the young gamma-ray pulsars, which all have $\dot{P}>10^{-16}$. However, for MSPs $\mu^{2} d$ can be large enough that $\dot{P}^{\text {int }}$ differs noticeably from the observed $\dot{P}$ values and quantities derived from $\dot{P}$ will also be affected.

From the literature we compiled proper motion measurements for 243 pulsars, all but one of which also have distance estimates and $\dot{P}$ measurements, and we calculated $\dot{P}^{\text {int }}$ and its uncertainties for those 242 pulsars. Of these, 69 have $P<30 \mathrm{~ms}$, and 20 are gamma-ray MSPs, listed in Table 6 . The magnitude of the correction is $\xi=\left(\dot{P}-\dot{P}^{\text {int }}\right) / \dot{P}$, or, equivalently, $\dot{E}^{\text {int }}=\dot{E}(1-\xi)$ since $\dot{E} \propto \dot{P}$. For $|\xi|$ greater than a few percent, $\dot{P}^{\text {shk }}>\left|\dot{P}^{\text {gal }}\right|$ and corrected $\dot{E}^{\text {int }}$ is less than the observed value. Hence flagging gamma-ray pulsar candidates that have large $\dot{E}$ selects some with lower $\dot{E}^{\text {int }}$, but we would not miss candidates by neglecting proper motion. For large Doppler corrections, the Galactic term is negligible, $\dot{P}^{\text {shk }} \gg \dot{P}^{\text {gal }}$, and we calculate only the uncertainty due to $\dot{P}^{\text {shk }}$. Unless otherwise noted, throughout this paper we use $\dot{P}^{\text {int }}$ from Table 6 to replace $\dot{P}$ and the derived quantities ( $\dot{E}, \tau$, et cetera) in the figures and tables. In Section 6.3 we discuss the Doppler correction's effect on the gamma-ray luminosity for a few cases.

\section{PROFILE CHARACTERIZATION}

\subsection{Gamma-Ray and Radio Light Curves}

Appendix A contains a small sample of gamma-ray pulse profiles (Figures 22(a)-(h)), overlaid with the radio profiles when available, and showing the fits described in Section 5.2, below. All pulse profiles are provided in the online material. We display gamma-ray light curves by computing a weighted histogram of gamma-ray rotational phases $\phi$. The error on the $i$ th histogram bin containing $N_{i}$ photons is estimated as $\sigma_{i}^{2}=1+\sum_{j=0}^{N_{i}} w_{j}^{2}$, using the weights $w_{j}$ defined in Equation (1). The " 1 " term mitigates a bias toward low histogram levels and $\sigma_{i}$ values caused by background-dominated bins. For these bins, the typical photon weight is very low, but the large weights of rare background photons near the pulsar position (within the PSF) can substantially increase the bin level. The additional 
Table 6

Millisecond Pulsar Distances and Spindown Doppler Corrections

\begin{tabular}{|c|c|c|c|c|c|c|c|c|c|c|}
\hline PSR & $\begin{array}{c}d \\
(\mathrm{pc})\end{array}$ & Method & $\operatorname{Ref}^{b}$ & $\begin{array}{c}\mu \\
\left(\operatorname{mas~yr}^{-1}\right)\end{array}$ & $\operatorname{Ref}^{c}$ & $\begin{array}{c}\dot{P}^{\text {int }} \\
\left(10^{-21}\right)\end{array}$ & $\begin{array}{c}\dot{P}^{\text {shk }} \\
\left(10^{-21}\right)\end{array}$ & $\begin{array}{c}\dot{P}^{\text {gal }} \\
\left(10^{-21}\right)\end{array}$ & $\begin{array}{c}\dot{E}^{\text {int }} \\
\left(10^{33} \mathrm{erg} \mathrm{s}^{-1}\right)\end{array}$ & $\begin{array}{c}\xi \\
(\%)\end{array}$ \\
\hline $\mathrm{J} 0023+0923$ & $690_{-110}^{+210}$ & DM & (1) & & & & & & & \\
\hline $\mathrm{J} 0030+0451$ & $280_{-60}^{+100}$ & $\mathrm{P}$ & (2) & $5.7 \pm 1.1$ & (1) & $10.7 \pm 0.1$ & 0.11 & -0.60 & $3.64 \pm 0.02$ & -5 \\
\hline J0034-0534 & $540 \pm 100$ & DM & (3) & $31.0 \pm 9.0$ & (2) & $2.9 \pm 1.4$ & 2.37 & -0.31 & $17.3 \pm 8.6$ & 41 \\
\hline J0101-6422 & $550_{-80}^{+90}$ & DM & (4) & $15.6 \pm 1.7$ & (3) & $4.4 \pm 0.2$ & 0.84 & -0.39 & $10.1 \pm 0.5$ & 9 \\
\hline J0102+4839 & $2320_{-430}^{+500}$ & DM & (1) & & & & & & & \\
\hline $\mathrm{J} 0218+4232$ & $2640_{-640}^{+1080}$ & DM & (5) & $5.0 \pm 6.0$ & (2) & $76.9 \pm 0.9$ & 0.37 & 0.09 & $243.2 \pm 2.8$ & 0.6 \\
\hline $\mathrm{J} 0340+4130$ & $1730 \pm 300$ & DM & (1) & & & & & & & \\
\hline $\mathrm{J} 0437-4715$ & $156 \pm 1$ & $\mathrm{P}$ & (2) & $141.3 \pm 0.1$ & (4) & $14.1 \pm 0.3$ & 43.59 & -0.40 & $2.9 \pm 0.1$ & 75 \\
\hline J0610-2100 & $3540_{-1000}^{+5460}$ & DM & (6) & $18.2 \pm 0.2$ & (5) & $1.2_{-1.1}^{+17.0}$ & 11.00 & 0.10 & $0.8_{-0.8}^{+11.7}$ & 90 \\
\hline J0613-0200 & $900_{-200}^{+400}$ & $\mathrm{P}$ & (2) & $10.8 \pm 0.2$ & (6) & $8.7_{-0.2}^{+0.3}$ & 0.77 & 0.08 & $12.0_{-0.2}^{+0.5}$ & 9 \\
\hline J0614-3329 & $1900_{-350}^{+440}$ & DM & (7) & & & & & & & \\
\hline $\mathrm{J} 0751+1807$ & $400_{-100}^{+200}$ & $\mathrm{P}$ & (2) & $6.0 \pm 2.0$ & (7) & $7.7 \pm 0.1$ & 0.12 & -0.02 & $7.2 \pm 0.1$ & 1 \\
\hline J1024-0719 & $390 \pm 40$ & $\mathrm{DM}^{\mathrm{a}}$ & (8) & $59.9 \pm 0.2$ & (5) & $1.6_{-1.4}^{+1.8}$ & 17.37 & -0.44 & $0.4_{-0.4}^{+0.5}$ & 92 \\
\hline J1124-3653 & $1720_{-360}^{+430}$ & $\mathrm{DM}$ & (1) & & & & & & & \\
\hline J1125-5825 & $2620 \pm 370$ & DM & (9) & & & & & & & \\
\hline $\mathrm{J} 1231-1411$ & $440 \pm 50$ & DM & (7) & $62.2 \pm 4.7$ & $(8)^{\mathrm{a}}$ & $6.5 \pm 2.9$ & 15.15 & -0.41 & $5.1 \pm 2.3$ & 70 \\
\hline $\mathrm{J} 1446-4701$ & $1460 \pm 220$ & DM & (10) & & & & & & & \\
\hline J1514-4946 & $940 \pm 120$ & DM & (4) & & & & & & & \\
\hline $\mathrm{J} 1600-3053$ & $1630_{-270}^{+310}$ & $\mathrm{DM}$ & (11) & $7.2 \pm 0.3$ & (6) & $8.6_{-0.1}^{+0.2}$ & 0.74 & 0.15 & $7.3 \pm 0.1$ & 9 \\
\hline $\mathrm{J} 1614-2230$ & $650 \pm 50$ & $\mathrm{P}$ & (12) & $36.5 \pm 0.2$ & (9) & $3.0 \pm 0.5$ & 6.65 & -0.003 & $3.8 \pm 0.6$ & 69 \\
\hline $\mathrm{J} 1658-5324$ & $930_{-130}^{+110}$ & DM & (4) & & & & & & & \\
\hline $\mathrm{J} 1713+0747$ & $1050_{-50}^{+60}$ & $\mathrm{P}$ & (2) & $6.30 \pm 0.01$ & (10) & $8.28_{-0.02}^{+0.03}$ & 0.46 & -0.21 & $3.42 \pm 0.01$ & 3 \\
\hline $\mathrm{J} 1741+1351$ & $1080_{-50}^{+40}$ & $\mathrm{P}$ & (13) & $11.71 \pm 0.01$ & (11) & $29.1 \pm 0.1$ & 1.35 & -0.20 & $21.76_{-0.05}^{+0.04}$ & 4 \\
\hline $\mathrm{J} 1744-1134$ & $417 \pm 17$ & $\mathrm{P}$ & (11) & $21.02 \pm 0.03$ & (6) & $7.0 \pm 0.1$ & 1.82 & 0.08 & $4.11 \pm 0.04$ & 21 \\
\hline $\mathrm{J} 1747-4036$ & $3390 \pm 760$ & DM & (4) & & & & & & & \\
\hline $\mathrm{J} 1810+1744$ & $2000_{-280}^{+310}$ & DM & (1) & & & & & & & \\
\hline $\mathrm{J} 1823-3021 \mathrm{~A}$ & $7600 \pm 400$ & $\mathrm{O}$ & (14) & & & & & & & \\
\hline J1858-2216 & $940_{-130}^{+200}$ & DM & (15) & & & & & & & \\
\hline J1902-5105 & $1180 \pm 210$ & DM & (4) & & & & & & & \\
\hline J1939+2134 & $3560 \pm 350$ & DM & (16) & $0.80 \pm 0.02$ & (12) & $105.5 \pm 0.1$ & 0.01 & -0.36 & $1096.6 \pm 0.5$ & -0.3 \\
\hline $\mathrm{J} 1959+2048$ & $2490_{-490}^{+160}$ & DM & (17) & $30.4 \pm 0.6$ & (13) & $8.1_{-1.8}^{+0.7}$ & 9.00 & -0.25 & $76.3_{-17.1}^{+6.4}$ & 52 \\
\hline $\mathrm{J} 2017+0603$ & $1570 \pm 150$ & DM & (18) & & & & & & & \\
\hline $\mathrm{J} 2043+1711$ & $1760_{-320}^{+150}$ & DM & $(1)$ & $13.0 \pm 2.0$ & (14) & $4.3 \pm 0.6$ & 1.72 & -0.35 & $12.7_{-1.8}^{+1.6}$ & 24 \\
\hline $\mathrm{J} 2047+1053$ & $2050_{-290}^{+320}$ & DM & (15) & & & & & & & \\
\hline $\mathrm{J} 2051-0827$ & $1040 \pm 150$ & DM & (19) & $7.3 \pm 0.4$ & (15) & $12.6 \pm 0.1$ & 0.61 & -0.47 & $5.43 \pm 0.05$ & 1 \\
\hline $\mathrm{J} 2124-3358$ & $300_{-50}^{+70}$ & $\mathrm{P}$ & $(2)$ & $52.3 \pm 0.3$ & $(6)$ & $11.2_{-1.6}^{+2.3}$ & 9.83 & -0.46 & $3.7_{-0.5}^{+0.8}$ & 46 \\
\hline $\mathrm{J} 2214+3000$ & $1540 \pm 180$ & DM & (7) & & & & & & & \\
\hline $\mathrm{J} 2215+5135$ & $3010_{-370}^{+330}$ & DM & (1) & & & & & & & \\
\hline $\mathrm{J} 2241-5236$ & $510 \pm 80$ & DM & (20) & & & & & & & \\
\hline $\mathrm{J} 2302+4442$ & $1190_{-230}^{+90}$ & DM & (18) & & & & & & & \\
\hline
\end{tabular}

Notes. Columns 2 and 3 give the distances for the 40 MSPs detected by Fermi, and the method used to find them: P-parallax; DM-from dispersion measure using the Cordes \& Lazio (2002) NE2001 model; O- other methods. For DM, the references in Column 4 give the DM measurement. For the 20 MSPs with a proper motion measurement, it is listed in Column 5, obtained from the reference in Column 6 . Columns 7 to 10 list the intrinsic $\dot{P}$ int , the contributions of the Shklovskii effect $\dot{P}^{\text {shk }}$ and of acceleration due to the Galactic rotation, $\dot{P}$ gal , and the intrinsic spindown power $\dot{E}^{\text {int }}$. The relative correction $\xi$ is defined from $\dot{E}^{\text {int }}=\dot{E}(1-\xi)$.

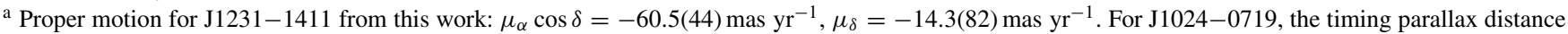
measurement gives negative spindown and we use instead the DM distance following Espinoza et al. (2013).

b Distance and DM references: (1) Hessels et al. 2011; (2) Verbiest et al. 2012; (3) Bailes et al. 1994; (4) Kerr et al. 2012; (5) Hobbs et al. 2004b; (6) Burgay et al. 2006; (7) Ransom et al. 2011; (8) Hotan et al. 2006; (9) Bates et al. 2011; (10) Keith et al. 2012; (11) Verbiest et al. 2009; (12) Lassus 2013; (13) P. C. C. Freire et al. (in preparation); (14) Kuulkers et al. 2003; (15) Ray et al. 2012; (16) Cognard et al. 1995; (17) Arzoumanian et al. 1994; (18) Cognard et al. 2011; (19) Doroshenko et al. 2001; (20) Keith et al. 2011.

c Proper motion references: (1) Abdo et al. 2009e; (2) Hobbs et al. 2005; (3) Kerr et al. 2012; (4) Deller et al. 2009; (5) Espinoza et al. 2013; (6) Verbiest et al. 2009; (7) Nice et al. 2005; (8) This work; (9) Lassus 2013; (10) Splaver et al. 2005; (11) P. C. C. Freire et al. (in preparation); (12) Cognard et al. 1995; (13) Arzoumanian et al. 1994; (14) Guillemot et al. 2012; (15) Lazaridis et al. 2011. 
term compensates for the spread due to the presence or absence of a single such photon. We choose the number of bins in the histogram according to the weighted $H$-test: 25 bins $(H<100)$; 50 bins $(100<H<1000)$; and 100 bins $(H>1000)$.

We estimate the background contribution from diffuse sources and neighboring point sources by computing the expectation value of $w$ under the hypothesis that the photon does not originate from the pulsar: $b \equiv \int_{0}^{1} d w w \times[1-f(w)] \approx$ $\sum_{j=0}^{N_{\gamma}} w_{j}-\sum_{j=0}^{N_{\gamma}} w_{j}^{2}$, where $f(w)$ is the probability distribution of the photon weights and we have used a Monte Carlo approximation to evaluate the integral. The corresponding background level for a weighted histogram, shown as a horizontal dashed line in the gamma-ray light curves, is $b / N_{\text {bins. }}$. The dominant error in this quantity arises from systematic errors in the normalization of the diffuse background. We estimate this contribution by increasing/reducing the overall normalization of the background by $6 \%$ (see Section 6.1 for discussion.)

For pulsars with known radio profiles, we also display by preference the flux density at $1400 \mathrm{MHz}$. When $1400 \mathrm{MHz}$ data are unavailable or highly scattered by the ISM, we include lower- or higher-frequency profiles, noting the frequency and provenance of the profile on the figures (Appendix A, and the online material).

The propagation of radio pulses through the dispersive ISM is delayed by $\Delta t \propto \mathrm{DM} v^{-2}$. This delay, as well as Roemertype delays associated with the configuration of the telescope relative to the solar system barycenter, are accounted for by TEMPO2, allowing precise alignment of radio and gammaray light curves. The absolute time at which $\phi \equiv 0$ is indicated in the pulsar par files, provided with the online material, by the parameters TZRMJD (giving the time of arrival of the zero phase), TZRFRQ (giving the frequency for which this time is correct), and TZRSITE (encoding the radio telescope/site of arrival).

The zero of phase-the fiducial phase-is ideally the rotational phase when the magnetic axis, the spin axis, and the line of sight lie in the same plane. For some pulsars, this phase can be identified by fitting the rotating vector model (Radhakrishnan \& Cooke 1969) to radio polarization position angle versus phase. However, radio pulses often cover too narrow a phase interval to constrain fits using the rotating vector model.

If radio emission from the polar cap is symmetric, the peak intensity can also be used as a proxy for the fiducial phase, and we adopt this approach here for radio-loud pulsars. In detail, this approach can fail if there is appreciable asymmetry in the radio beam or profile evolution with frequency. Additionally, for pulsars with radio interpulses and for some MSPs, we observe emission from the field lines from both poles and must choose with which to associate the fiducial phase. In these cases, we generally choose the hemisphere furthest separated in phase from the gamma rays, consistent with an interpretation of gamma-ray emission arising from the outer magnetosphere. Finally, some pulsars (e.g., J0034-0534 shown in Figure 22(a)) exhibit a clear double symmetry in the radio light curve. In these cases, we choose a fiducial point near the point of symmetry. We note which prescription we have followed in Tables 7 and 8 with a "p" (fiducial point at peak intensity), "h" (fiducial point from hemisphere opposite to peak intensity), "s" (fiducial point placed at point of symmetry rather than peak). For radio-quiet pulsars, we put the first gamma-ray peak (identified by looking for sharp rises and bridge emission) at $\phi=0.1$ for display purposes.

\subsection{Gamma-Ray Light Curve Fitting}

Generally, the gamma-ray light curve of a pulsar can be represented by a wrapped probability density function (pdf) of $\phi \in[0,1)$. A compact approximation of the true pdf for photon phases can be constructed as a linear combination of $N$ unimodal, possibly skew distributions:

$$
f(\phi)=\sum_{i=1}^{N} n_{i} g_{i}(\phi)+\left(1-\sum_{i=1}^{N} n_{i}\right),
$$

where each of the $g_{i}$ is an individually normalized distribution and $1-\sum_{i=1}^{N} n_{i} \leqslant 1$ is a uniform distribution representing an unpulsed component. To explicitly enforce normalization, we use spherical polar coordinates lying within the unit sphere as internal parameters. With three components, e.g., the normalizations $n_{i}$ are given in terms of the internal parameters $\chi_{i}$ as $n_{1}=\sin \chi_{1} \cos \chi_{2} ; n_{2}=\sin \chi_{1} \sin \chi_{2} \cos \chi_{3}$; $n_{3}=\sin \chi_{1} \sin \chi_{2} \sin \chi_{3}$.

Although generalized wrapped distributions exist, analytic forms for such distributions are typically unavailable. Instead we adopt well-known pdfs, viz. the Gaussian (normal) and Lorentzian (Cauchy) distributions, by wrapping them onto a circle:

$$
g(\phi)=\sum_{i=-\infty}^{+\infty} g^{\prime}(\phi+i)
$$

where $g^{\prime}$ is defined on the real line and $g$ on the circle. For practical reasons this sum must be truncated, and we define a truncated, wrapped distribution as

$$
g_{T}(\phi, N)=\sum_{i=-N}^{+N} g^{\prime}(\phi+i)+\left(1-\int_{-N}^{+N} g^{\prime}(x) d x\right),
$$

i.e., we approximate the tails as a uniform distribution. In the fits discussed below, $N \equiv 10$.

Because the peaks of gamma-ray light curves may have a caustic origin, asymmetric distributions are needed to model their fast rise and slow fall. We generalize the symmetric Gaussian and Lorentzian distributions by matching two distributions with differing width parameters, $\sigma_{1}$ and $\sigma_{2}$, at the maximum, $x_{0}$. Defining $z \equiv\left(x-x_{0}\right) / \sigma$ with $\sigma=\sigma_{1}$ if $z \leqslant 0$ and $\sigma=\sigma_{2}$ otherwise, the functional forms of the resulting distributions on the real line are

$$
\begin{gathered}
g^{\prime}(x)=\frac{2}{\pi\left(\sigma_{1}+\sigma_{2}\right)\left(1+z^{2}\right)} \text { (Lorentzian), } \\
g^{\prime}(x)=\sqrt{\frac{2}{\pi}} \frac{\exp \left(\frac{-z^{2}}{2}\right)}{\sigma_{1}+\sigma_{2}} \text { (Gaussian). }
\end{gathered}
$$

We employ maximum likelihood to determine the best-fit parameters of the mixture distribution. If $w_{i}$ is a probability that a photon originates from the pulsar, then the logarithm of the likelihood is

$$
\log \mathcal{L}=\sum_{j=1}^{N_{\gamma}} \log \left[w_{i} f\left(\phi_{i}\right)+\left(1-w_{i}\right)\right]
$$

For large data sets $\left(N_{\gamma}>10^{4}\right)$ we speed up the computation by binning $f(\phi)$ to 512 values. 
Table 7

Pulse Shape Parameters of Young LAT-detected Pulsars

\begin{tabular}{|c|c|c|c|c|c|}
\hline $\mathrm{PSR}^{\mathrm{a}}$ & Peaks & $\begin{array}{c}\text { Radio Lag } \\
\delta\end{array}$ & $\begin{array}{l}\text { Shift } \\
\text { Method }\end{array}$ & $\begin{array}{c}\text { Gamma-peak Separation } \\
\Delta\end{array}$ & $\begin{array}{c}\text { Off-peak Definition } \\
\phi\end{array}$ \\
\hline $\mathrm{J} 0007+7303$ & 2 & $\ldots$ & & $0.216 \pm 0.005$ & $0.50-0.86$ \\
\hline $\mathrm{J} 0106+4855$ & 2 & $0.062 \pm 0.002$ & $\mathrm{p}$ & $0.487 \pm 0.003$ & $0.14-0.50,0.75-0.01$ \\
\hline J0205+6449 & 2 & $0.075 \pm 0.004$ & $\mathrm{p}$ & $0.503 \pm 0.004$ & $0.64-0.99$ \\
\hline J0248+6021 & 1 & $0.336 \pm 0.017$ & $\mathrm{p}$ & $\ldots$ & $0.61-0.18$ \\
\hline $\mathrm{J} 0357+3205$ & 1 & $\ldots$ & & $\ldots$ & $0.39-0.90$ \\
\hline $\mathrm{J} 0534+2200$ & 2 & $0.109 \pm 0.001$ & o & $0.407 \pm 0.001$ & $0.71-0.99$ \\
\hline J0622+3749 & 2 & $\ldots$ & & $0.457 \pm 0.034$ & $0.37-0.52,0.69-0.89$ \\
\hline $\mathrm{J} 0631+1036$ & 1 & $0.497 \pm 0.022$ & $\mathrm{~s}$ & $\ldots$ & $0.63-0.17$ \\
\hline $\mathrm{J} 0633+0632$ & 2 & $\ldots$ & & $0.476 \pm 0.003$ & $0.24-0.52,0.67-1.00$ \\
\hline J0633+1746 & 2 & $\ldots$ & & $0.508 \pm 0.001$ & $0.85-0.95$ \\
\hline J0659+1414 & 1 & $0.224 \pm 0.010$ & $\mathrm{p}$ & $\ldots$ & $0.40-0.04$ \\
\hline J0729-1448 & 1 & $0.577 \pm 0.010$ & $\mathrm{p}$ & $\ldots$ & $0.70-0.47$ \\
\hline J0734-1559 & 1 & $\ldots$ & & $\ldots$ & $0.28-0.84$ \\
\hline J0742-2822 & 1 & $0.627 \pm 0.005$ & $\mathrm{p}$ & $\ldots$ & $0.74-0.01,0.01-0.48$ \\
\hline J0835-4510 & 3 & $0.129 \pm 0.001$ & $\mathrm{p}$ & $0.433 \pm 0.001$ & $0.81-0.03$ \\
\hline J0908-4913 & 2 & $0.102 \pm 0.005$ & $\mathrm{p}$ & $0.501 \pm 0.006$ & $0.66-0.04,0.17-0.54$ \\
\hline J0940-5428† & 1 & $0.451 \pm 0.035$ & $\mathrm{~s}$ & $\ldots$ & $0.59-0.19$ \\
\hline J1016-5857 & 2 & $0.143 \pm 0.003$ & $\mathrm{~s}$ & $0.423 \pm 0.004$ & $0.64-0.04$ \\
\hline J1019-5749 & 1 & $0.482 \pm 0.010$ & $\mathrm{p}$ & $\ldots$ & $0.74-0.36$ \\
\hline $\mathrm{J} 1023-5746$ & 2 & $\ldots$ & & $0.474 \pm 0.002$ & $0.76-0.02$ \\
\hline J1028-5819 & 2 & $0.195 \pm 0.001$ & $\mathrm{p}$ & $0.475 \pm 0.001$ & $0.75-0.08$ \\
\hline J1044-5737 & 2 & $\ldots$ & & $0.373 \pm 0.004$ & $0.56-0.98$ \\
\hline J1048-5832 & 2 & $0.125 \pm 0.001$ & $\mathrm{~s}$ & $0.426 \pm 0.001$ & $0.65-0.02$ \\
\hline J1057-5226 & 3 & $0.304 \pm 0.003$ & $\mathrm{sh}$ & $0.307 \pm 0.004$ & $0.72-0.14$ \\
\hline J1105-6107 & 2 & $0.110 \pm 0.001$ & $\mathrm{~s}$ & $0.504 \pm 0.006$ & $0.74-0.04,0.22-0.47$ \\
\hline J1112-6103 & 2 & $0.192 \pm 0.007$ & $\mathrm{p}$ & $0.457 \pm 0.013$ & $0.79-0.04,0.31-0.56$ \\
\hline J1119-6127 & 2 & $0.285 \pm 0.015$ & $\mathrm{p}$ & $0.204 \pm 0.020$ & $0.59-0.18$ \\
\hline J1124-5916 & 2 & $0.141 \pm 0.003$ & $\mathrm{p}$ & $0.499 \pm 0.004$ & $0.69-0.05$ \\
\hline J1135-6055 & 1 & $\ldots$ & & $\ldots$ & $0.49-0.92$ \\
\hline J1357-6429 & 1 & $0.359 \pm 0.028$ & $\mathrm{p}$ & $\ldots$ & $0.64-0.13$ \\
\hline J1410-6132 & 2 & $0.959 \pm 0.023$ & $\mathrm{p}$ & $0.458 \pm 0.037$ & $0.04-0.24,0.55-0.85$ \\
\hline $\mathrm{J} 1413-6205$ & 2 & $\ldots$ & & $0.372 \pm 0.003$ & $0.57-0.01$ \\
\hline $\mathrm{J} 1418-6058 \star$ & 2 & $\ldots$ & & $0.467 \pm 0.003$ & $0.65-0.93$ \\
\hline J1420-6048 & 2 & $0.196 \pm 0.011$ & $\mathrm{~s}$ & $0.312 \pm 0.015$ & $0.63-0.12$ \\
\hline J1429-5911 & 2 & $\ldots$ & & $0.479 \pm 0.004$ & $0.28-0.40$ \\
\hline $\mathrm{J} 1459-6053 \star$ & 1 & $\ldots$ & & $\ldots$ & $0.59-0.91$ \\
\hline $\mathrm{J} 1509-5850$ & 2 & $0.271 \pm 0.011$ & $\mathrm{p}$ & $0.264 \pm 0.013$ & $0.64-0.14$ \\
\hline J1513-5908 & 1 & $0.325 \pm 0.055$ & $\mathrm{p}$ & $\ldots$ & $0.53-0.15$ \\
\hline $\mathrm{J} 1531-5610 \dagger$ & 1 & $0.413 \pm 0.035$ & $\mathrm{~s}$ & $\ldots$ & $0.57-0.23$ \\
\hline J1620-4927 & 2 & $\ldots$ & & $0.231 \pm 0.030$ & $0.51-0.95$ \\
\hline $\mathrm{J} 1648-4611$ & 2 & $0.261 \pm 0.010$ & $\mathrm{p}$ & $0.298 \pm 0.082$ & $0.64-0.17$ \\
\hline $\mathrm{J} 1702-4128$ & 1 & $0.397 \pm 0.038$ & $\mathrm{p}$ & $\ldots$ & $0.57-0.17$ \\
\hline J1709-4429 & 2 & $0.239 \pm 0.001$ & $\mathrm{p}$ & $0.244 \pm 0.002$ & $0.72-0.07$ \\
\hline $\mathrm{J} 1718-3825$ & 1 & $0.397 \pm 0.009$ & $\mathrm{~s}$ & $\ldots$ & $0.64-0.09$ \\
\hline $\mathrm{J} 1730-3350$ & 2 & $0.128 \pm 0.007$ & $\mathrm{p}$ & $0.419 \pm 0.007$ & $0.63-0.04,0.20-0.38$ \\
\hline J1732-3131 & 2 & $\ldots$ & & $0.419 \pm 0.002$ & $0.59-0.95$ \\
\hline J1741-2054 & 2 & $0.074 \pm 0.006$ & $\mathrm{~s}$ & $0.244 \pm 0.011$ & $0.44-0.96$ \\
\hline J1746-3239 & 2 & $\ldots$ & & $0.179 \pm 0.019$ & $0.44-0.02$ \\
\hline J1747-2958 & 2 & $0.181 \pm 0.003$ & $\mathrm{p}$ & $0.392 \pm 0.005$ & $0.64-0.08$ \\
\hline $\mathrm{J} 1801-2451$ & 2 & $0.060 \pm 0.005$ & $\mathrm{p}$ & $0.496 \pm 0.020$ & $0.13-0.48,0.61-0.97$ \\
\hline J1803-2149 & 2 & $\ldots$ & & $0.394 \pm 0.009$ & $0.59-0.03$ \\
\hline J1809-2332 & 2 & $\ldots$ & & $0.358 \pm 0.002$ & $0.55-0.93$ \\
\hline J1813-1246 & 2 & $\ldots$ & & $0.489 \pm 0.010$ & $0.74-0.98$ \\
\hline J1826-1256 & 2 & $\ldots$ & & $0.480 \pm 0.001$ & $0.67-0.98$ \\
\hline J1833-1034 & 2 & $0.153 \pm 0.002$ & $\mathrm{p}$ & $0.447 \pm 0.004$ & $0.69-0.10$ \\
\hline $\mathrm{J} 1835-1106$ & 2 & $0.139 \pm 0.006$ & $\mathrm{p}$ & $0.421 \pm 0.011$ & $0.64-0.04$ \\
\hline J1836+5925 & 2 & $\ldots$ & & $0.537 \pm 0.006$ & $0.76-0.92$ \\
\hline $\mathrm{J} 1838-0537 \star$ & 2 & $\ldots$ & & $0.298 \pm 0.014$ & $0.51-0.01$ \\
\hline J1846+0919 & 2 & $\ldots$ & & $0.244 \pm 0.022$ & $0.43-0.95$ \\
\hline J1907+0602 & 2 & $0.209 \pm 0.003$ & $\mathrm{p}$ & $0.389 \pm 0.004$ & $0.69-0.12$ \\
\hline $\mathrm{J} 1952+3252$ & 3 & $0.161 \pm 0.002$ & $\mathrm{p}$ & $0.478 \pm 0.003$ & $0.71-0.06$ \\
\hline J1954+2836 & 2 & $\ldots$ & & $0.456 \pm 0.004$ & $0.67-0.02$ \\
\hline $\mathrm{J} 1957+5033$ & 1 & $\ldots$ & & $\ldots$ & $0.46-0.93$ \\
\hline $\mathrm{J} 1958+2846$ & 2 & $\ldots$ & & $0.454 \pm 0.004$ & $0.65-0.04$ \\
\hline
\end{tabular}


Table 7

(Continued)

\begin{tabular}{|c|c|c|c|c|c|}
\hline $\mathrm{PSR}^{\mathrm{a}}$ & Peaks & $\begin{array}{c}\text { Radio Lag } \\
\quad \delta\end{array}$ & $\begin{array}{l}\text { Shift } \\
\text { Method }\end{array}$ & $\begin{array}{c}\text { Gamma-peak Separation } \\
\Delta\end{array}$ & $\begin{array}{c}\text { Off-peak Definition } \\
\phi\end{array}$ \\
\hline $\mathrm{J} 2021+3651$ & 2 & $0.132 \pm 0.001$ & $\mathrm{p}$ & $0.478 \pm 0.001$ & $0.73-0.99$ \\
\hline $\mathrm{J} 2021+4026$ & 2 & $\ldots$ & & $0.441 \pm 0.016$ & $0.24-0.40$ \\
\hline $\mathrm{J} 2028+3332$ & 2 & $\ldots$ & & $0.451 \pm 0.003$ & $0.57-0.97$ \\
\hline $\mathrm{J} 2030+3641$ & 2 & $0.269 \pm 0.010$ & $\mathrm{p}$ & $0.309 \pm 0.014$ & $0.67-0.18$ \\
\hline $\mathrm{J} 2030+4415$ & 2 & $\ldots$ & & $0.505 \pm 0.007$ & $0.65-0.01$ \\
\hline $\mathrm{J} 2032+4127$ & 2 & $0.099 \pm 0.001$ & $\mathrm{p}$ & $0.516 \pm 0.001$ & $0.22-0.55,0.68-1.00$ \\
\hline $\mathrm{J} 2043+2740$ & 2 & $0.132 \pm 0.007$ & $\mathrm{p}$ & $0.432 \pm 0.010$ & $0.63-0.05$ \\
\hline $\mathrm{J} 2055+2539 \star$ & 2 & $\ldots$ & & $0.113 \pm 0.017$ & $0.39-0.89$ \\
\hline $\mathrm{J} 2111+4606 \star$ & 2 & $\ldots$ & & $0.337 \pm 0.011$ & $0.53-0.01$ \\
\hline $\mathrm{J} 2139+4716$ & 1 & $\ldots$ & & $\ldots$ & $0.25-0.90$ \\
\hline $\mathrm{J} 2229+6114$ & 2 & $0.187 \pm 0.007$ & $\mathrm{p}$ & $0.299 \pm 0.008$ & $0.68-0.10$ \\
\hline $\mathrm{J} 2238+5903$ & 2 & $\ldots$ & & $0.502 \pm 0.002$ & $0.68-0.02$ \\
\hline $\mathrm{J} 2240+5832$ & 2 & $0.118 \pm 0.014$ & $\mathrm{p}$ & $0.476 \pm 0.014$ & $0.70-0.05,0.16-0.46$ \\
\hline
\end{tabular}

Notes. Column 2 gives the gamma-ray peak multiplicity. Columns 3 and 5 give the gamma-radio phase lag $\delta$ and separation $\Delta$ between the gamma-ray peaks. Column 4 gives the method used to define the radio fiducial phase: "p"-peak radio intensity; "s"- point of symmetry in the radio profile; "h"—opposite hemisphere (0.5 phase shift from "p" or "s" point). PSR J0534+2200 (the Crab pulsar) is exceptional, and we align its profile with the low frequency component observed by Moffett \& Hankins (1996) and denote this method "o" for "other." Column 6 gives the off-peak interval definition from Section 7.

a A dagger $(\dagger)$ means the pulse profile fit is unreliable. A star $(\star)$ means that systematic offset uncertainties from the radio timing residuals (TRES variable in TEMPO) are between 10 and 19 milliperiods.

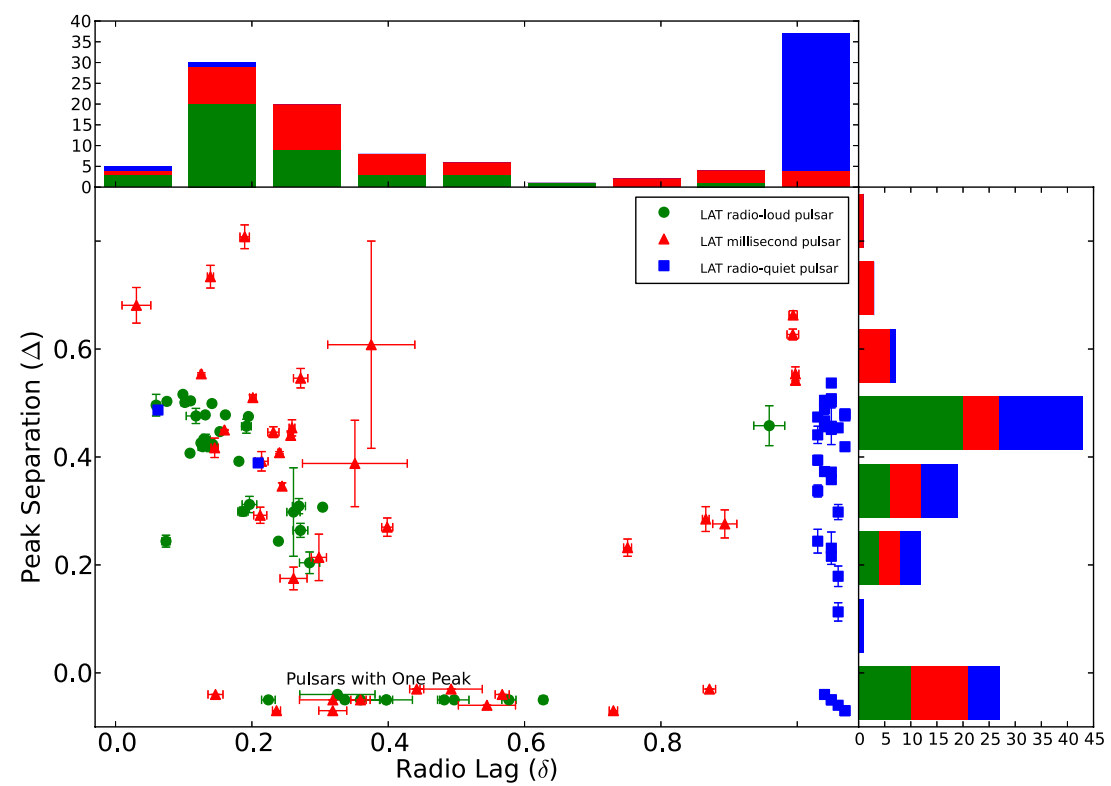

Figure 5. Phase lag $\delta$ of the gamma peak relative to the fiducial phase vs. the phase separation $\Delta$ between the gamma-ray peaks. The artificial staggering of the points along the horizontal axis (single-peaked pulsars) and the right-hand vertical axis (pulsars with no radio detection) is to enhance clarity. The markers are the same as in Figure 1, as is the color code of the histograms projected onto the axes.

(A color version of this figure is available in the online journal.)

Most LAT light curves can be modeled with good fidelity by one or two two-sided narrow peak distributions and a single broad bridge component. From these fits, we determine the following quantities: $N_{\text {peaks }}$, the number of non-bridge components; $\delta$, the offset of the mode of the leading peak from the fiducial phase (see above); and $\Delta$, the difference between the modes of the leading and trailing peak (for $N_{\text {peaks }}>1$ ). These parameters appear in Tables 7 and 8 . The strong correlation between $\Delta$ and $\delta$ in Figure 5, as well as the dependence on spindown power (Figure 6) are discussed in Section 10. The peak widths are included in the online material.
The uncertainty on $\delta$ is estimated by combining in quadrature the statistical uncertainty on the position of the relevant gammaray peak with that incurred from uncertainty in the DM. The statistical uncertainty naturally includes a contribution from uncertainty in the timing solution (the TEMPO TRES quantity) which serves to smear out light curve features by a characteristic width $\delta \phi \approx \operatorname{TRES} / P$. The statistical uncertainty on $\Delta$ is determined by the sum in quadrature of the position uncertainty of the relevant gamma-ray peaks.

The representation of the light curve (Gaussian versus Lorentzian, presence or absence of additional components) affects the results for both $\delta$ and $\Delta$. In addition to the 
Table 8

Pulse Shape Parameters of LAT-detected Millisecond Pulsars

\begin{tabular}{|c|c|c|c|c|c|}
\hline $\mathrm{PSR}^{\mathrm{a}}$ & Peaks & $\begin{array}{c}\text { Radio Lag } \\
\quad \delta\end{array}$ & $\begin{array}{l}\text { Shift } \\
\text { Method }\end{array}$ & $\begin{array}{c}\text { Gamma-peak Separation } \\
\Delta\end{array}$ & $\begin{array}{c}\text { Off-peak Definition } \\
\phi \phi\end{array}$ \\
\hline J0023+0923 & 2 & $0.375 \pm 0.064$ & $\mathrm{p}$ & $0.608 \pm 0.192$ & $0.60-0.80$ \\
\hline J0030+0451 & 2 & $0.160 \pm 0.001$ & $\mathrm{p}$ & $0.450 \pm 0.001$ & $0.72-0.08$ \\
\hline J0034-0534 & 2 & $0.866 \pm 0.005$ & $\mathrm{~s}$ & $0.285 \pm 0.023$ & $0.28-0.75$ \\
\hline J0101-6422 & 2 & $0.145 \pm 0.005$ & sh & $0.417 \pm 0.018$ & $0.85-0.09$ \\
\hline J0102+4839 & 2 & $0.259 \pm 0.004$ & $\mathrm{p}$ & $0.454 \pm 0.015$ & $0.83-0.17,0.32-0.59$ \\
\hline J0218+4232 & 2 & $0.351 \pm 0.077$ & $\mathrm{~s}$ & $0.388 \pm 0.080$ & $0.84-0.02,0.02-0.23$ \\
\hline $\mathrm{J} 0340+4130$ & 2 & $0.751 \pm 0.006$ & $\mathrm{p}$ & $0.232 \pm 0.016$ & $0.11-0.62$ \\
\hline J0437-4715 & 1 & $0.442 \pm 0.010$ & $\mathrm{p}$ & $\ldots$ & $0.60-0.13$ \\
\hline J0610-2100 & 1 & $0.236 \pm 0.006$ & $\mathrm{~h}$ & & $0.98-0.20$ \\
\hline J0613-0200 & 2 & $0.261 \pm 0.020$ & $\mathrm{p}$ & $0.175 \pm 0.021$ & $0.60-0.10$ \\
\hline J0614-3329 & 2 & $0.126 \pm 0.002$ & $\mathrm{~s}$ & $0.554 \pm 0.002$ & $0.40-0.52$ \\
\hline J0751+1807 & 3 & $0.398 \pm 0.008$ & $\mathrm{~s}$ & $0.270 \pm 0.017$ & $0.77-0.03,0.03-0.32$ \\
\hline J1024-0719 & 1 & $0.492 \pm 0.046$ & $\mathrm{p}$ & $\ldots$ & $0.88-0.37$ \\
\hline J1124-3653 & 2 & $0.298 \pm 0.011$ & $\mathrm{~s}$ & $0.214 \pm 0.043$ & $0.88-0.19$ \\
\hline $\mathrm{J} 1125-5825 \dagger$ & 1 & $0.645 \pm 0.002$ & $\mathrm{p}$ & $\ldots$ & $0.74-0.48$ \\
\hline $\mathrm{J} 1231-1411$ & 3 & $0.241 \pm 0.002$ & $\mathrm{p}$ & $0.408 \pm 0.002$ & $0.72-0.12$ \\
\hline J1446-4701 & 1 & $0.319 \pm 0.021$ & $\mathrm{p}$ & $\ldots$ & $0.65-0.21$ \\
\hline J1514-4946 & 2 & $0.214 \pm 0.009$ & $\mathrm{~s}$ & $0.392 \pm 0.018$ & $0.69-0.15$ \\
\hline $\mathrm{J} 1600-3053$ & 1 & $0.147 \pm 0.011$ & $\mathrm{p}$ & $\ldots$ & $0.51-0.09$ \\
\hline $\mathrm{J} 1614-2230$ & 2 & $0.201 \pm 0.005$ & $\mathrm{p}$ & $0.510 \pm 0.005$ & $0.78-0.14$ \\
\hline J1658-5324 & 1 & $0.359 \pm 0.014$ & $\mathrm{~s}$ & $\ldots$ & $0.64-0.26$ \\
\hline $\mathrm{J} 1713+0747$ & 1 & $0.319 \pm 0.049$ & $\mathrm{p}$ & $\ldots$ & $0.67-0.00,0.00-0.19$ \\
\hline $\mathrm{J} 1741+1351$ & 1 & $0.730 \pm 0.006$ & $\mathrm{p}$ & $\ldots$ & $0.91-0.59$ \\
\hline J1744-1134 & 2 & $0.189 \pm 0.007$ & $\mathrm{sh}$ & $0.808 \pm 0.022$ & $0.05-0.10$ \\
\hline J1747-4036 & 2 & $0.031 \pm 0.021$ & $\mathrm{p}$ & $0.681 \pm 0.033$ & $0.24-0.44,0.73-0.88$ \\
\hline J1810+1744 & 2 & $0.894 \pm 0.018$ & $\mathrm{p}$ & $0.276 \pm 0.026$ & $0.23-0.68$ \\
\hline $\mathrm{J} 1823-3021 \mathrm{~A}$ & 2 & $0.993 \pm 0.009$ & $\mathrm{p}$ & $0.627 \pm 0.010$ & $0.08-0.55$ \\
\hline $\mathrm{J} 1858-2216 \dagger$ & 1 & $0.727 \pm 0.011$ & $\mathrm{p}$ & $\ldots$ & $0.16-0.66$ \\
\hline J1902-5105 & 2 & $0.994 \pm 0.006$ & $\mathrm{p}$ & $0.663 \pm 0.008$ & $0.70-0.93$ \\
\hline $\mathrm{J} 1939+2134$ & 2 & $0.997 \pm 0.004$ & $\mathrm{p}$ & $0.542 \pm 0.009$ & $0.08-0.47,0.59-0.95$ \\
\hline $\mathrm{J} 1959+2048$ & 2 & $0.997 \pm 0.005$ & $\mathrm{p}$ & $0.554 \pm 0.013$ & $0.19-0.38$ \\
\hline $\mathrm{J} 2017+0603$ & 2 & $0.212 \pm 0.010$ & $\mathrm{p}$ & $0.292 \pm 0.015$ & $0.60-0.12$ \\
\hline $\mathrm{J} 2043+1711$ & 2 & $0.231 \pm 0.008$ & $\mathrm{p}$ & $0.446 \pm 0.010$ & $0.73-0.10$ \\
\hline $\mathrm{J} 2047+1053$ & 1 & $0.567 \pm 0.010$ & $\mathrm{p}$ & $\ldots$ & $0.16-0.49$ \\
\hline J2051-0827 & 1 & $0.545 \pm 0.042$ & $\mathrm{p}$ & $\ldots$ & $0.80-0.28$ \\
\hline $\mathrm{J} 2124-3358$ & 1 & $0.871 \pm 0.009$ & $\mathrm{p}$ & $\ldots$ & $0.01-0.51$ \\
\hline $\mathrm{J} 2214+3000$ & 2 & $0.271 \pm 0.010$ & $\mathrm{p}$ & $0.546 \pm 0.018$ & $0.09-0.24,0.63-0.73$ \\
\hline $\mathrm{J} 2215+5135$ & 2 & $0.257 \pm 0.004$ & $\mathrm{p}$ & $0.440 \pm 0.008$ & \\
\hline J2241-5236 & 3 & $0.139 \pm 0.004$ & $\mathrm{p}$ & $0.638 \pm 0.031$ & $0.60-0.67$ \\
\hline $\mathrm{J} 2302+4442$ & 2 & $0.244 \pm 0.003$ & $\mathrm{~s}$ & $0.346 \pm 0.006$ & $0.70-0.18$ \\
\hline
\end{tabular}

Notes. Column 2 gives the gamma-ray peak multiplicity. Columns 3 and 5 give the phase lag $\delta$ and peak separation $\Delta$. Column 4 gives the method used to define the radio fiducial phase: "p" - peak radio intensity; "s" - point of symmetry in the radio profile; "h"—opposite hemisphere ( 0.5 phase shift from "p" or "s" point). The off-peak phase interval (Section 7) is in column 6 .

a A dagger $(\dagger)$ means the pulse profile fit is unreliable.

uncertainties above, we estimate a "model" uncertainty of $\sim 0.01$ in phase.

\section{SPECTRAL ANALYSES}

Most models of pulsar gamma-ray emission predict that the spectrum in the LAT energy range should be dominated by curvature radiation, in the radiation-reaction limited regime (e.g., Muslimov \& Harding 2004). This mechanism predicts that pulsar spectra should be exponentially cut off near energies of a few $\mathrm{GeV}$. The detection of pulsed emission above $100 \mathrm{GeV}$ from the Crab (see Sections 8.4 and 10) suggests that, for some pulsars, either an additional component becomes dominant above the cutoff or a different mechanism, e.g., inverse Compton scattering, may be responsible for the LAT emission. For the purposes of this catalog, we assume that all gamma-ray pulsars have a cutoff spectrum but test for any deviations from this model and report the pulsars that exhibit a different spectral shape.

$\mathrm{GeV}$ emission from pulsars is largely modulated at the rotational period, with the emission concentrated in one or more narrow peaks. Depending on the viewing geometry and emission model, a pulsar can have a $100 \%$ duty cycle and significant magnetospheric emission can also exist away from the peaks. Young pulsars power PWNe, some of which are detected with the LAT, spatially overlapping their associated pulsars. PWNlike emission could be confused with the magnetospheric signal but would not be modulated at the rotational period. Significant contamination from a PWN-like background source needs to be accounted for to properly study the pulsar emission. 


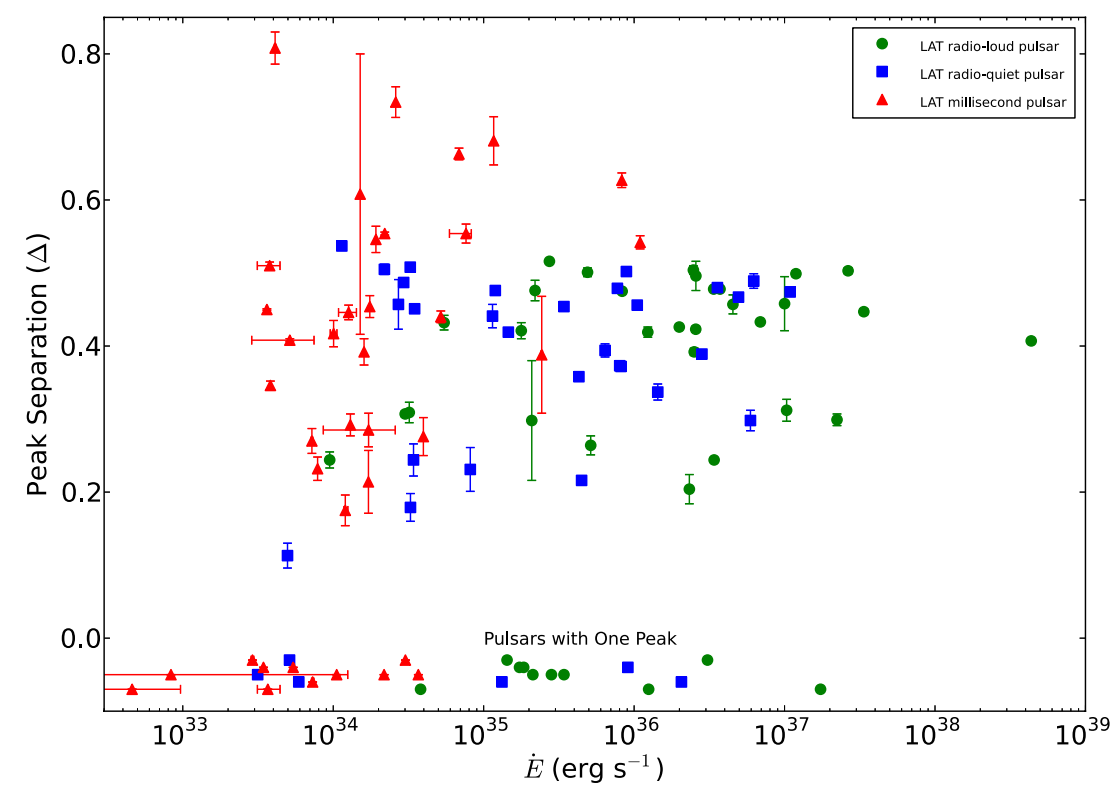

Figure 6. Phase separation $\Delta$ between gamma-ray peaks vs. the Shklovskii-corrected spindown power. The markers are the same as in Figure 1 .

(A color version of this figure is available in the online journal.)

With this goal, we first analyzed the off-peak phase intervals as described in Section 7 and characterized significantly detected off-peak emission, to identify constant magnetospheric emission or PWN-like background emission. In this section, we use the data with all rotation phases to characterize the magnetospheric emission, removing background PWN-like contamination, and report the phase-averaged spectra.

\subsection{Spectral Method}

For spectral analysis we used the data set described in Section 2 for phase-averaged fits. We evaluated the point-source detection significance and the spectral parameters for each LAT pulsar in this catalog with an analysis similar to that performed for 2FGL using the standard LAT Science Tools package. ${ }^{97}$ The spectrum of each LAT pulsar was modeled as a power law with an exponential cutoff,

$$
\frac{d N}{d E}=K\left(\frac{E}{E_{0}}\right)^{-\Gamma} \exp \left(-\frac{E}{E_{\text {cut }}}\right)^{b} .
$$

The four parameters are the normalization factor $(K)$, the photon index at low energy $(\Gamma)$, the cutoff energy $\left(E_{\text {cut }}\right)$, and a parameter representing the sharpness of the cutoff $(b)$ which is fixed to 1 in the default fit. The energy $E_{0}$ at which $K$ is defined is arbitrary; thus, we used the 2FGL pivot energy when it exists and $1 \mathrm{GeV}$ otherwise. In the likelihood analysis we constrained $\Gamma$ to be between 0 and 5 and $E_{\text {cut }}$ to be between 0.1 and $100 \mathrm{GeV}$.

We constructed models including all 2FGL sources within $20^{\circ}$ of each pulsar but only the spectral parameters of point sources within $8^{\circ}$ were left free. For sources known to be significantly extended we used the same spatial templates as 2FGL and fixed the spectral parameters to the 2FGL values. For pulsars with no 2FGL counterpart and an unassociated source within 0.1 of the pulsar, we moved the source to the timing position; otherwise, we added a new point source to the model at the timing position. The study of the off-peak phase interval in Section 7 yielded four pulsars with wind-like

\footnotetext{
97 http://fermi.gsfc.nasa.gov/ssc/data/analysis/scitools/overview.html
}

emission ("W"-type), and four pulsars where the origin of the off-peak emission is unidentified ("U"-type) but the emission appears spatially extended without evidence of a spectral cutoff, likely due to poorly modeled diffuse emission. We added a new point source to the model for each of these eight pulsars. We used the same models for diffuse gamma-ray emission as 2FGL to account for the Galactic, isotropic, and Earth limb emission: gal_2yearp7v6_v0.fits, iso_p7v6source.txt, limb_2year_P76_source_v0_smooth.txt, and limb_ smooth.fits. These are available from the Fermi Science Support Center. ${ }^{98}$

For each pulsar, we selected a $20^{\circ} \times 20^{\circ}$ square region, centered on the timing position, for a binned maximum likelihood gtlike analysis using the pyLikelihood python module included with the Fermi Science Tools. The best-fit parameters are obtained by maximizing the log-likelihood surface that represents the input model using the MINUIT2 fitting engine. ${ }^{99}$ The statistical uncertainties on the parameters were estimated from the quadratic development of the log-likelihood around the best fit. We first performed a fit with a weak convergence criterion and evaluated the point-source detection significance (the "Test Statistic" TS; Mattox et al. 1996) for each point source that had free parameters, except the pulsar of interest. We removed all point sources with TS $<2$ and re-optimized the fit with a stricter convergence criterion.

We report the maximum likelihood values of $\Gamma$ and $E_{\text {cut }}$ from the phase-averaged analysis and TS values in Tables 9 and 10 for young and millisecond pulsars, respectively. In addition, we report the integrated photon and energy fluxes in the 0.1 to $100 \mathrm{GeV}$ energy band ( $F_{100}$ and $G_{100}$, respectively), obtained from the fits as

$$
F_{100}=\int_{100 \mathrm{MeV}}^{100 \mathrm{GeV}} \frac{d N}{d E} d E, \quad G_{100}=\int_{100 \mathrm{MeV}}^{100 \mathrm{GeV}} E \frac{d N}{d E} d E .
$$

\footnotetext{
98 http://fermi.gsfc.nasa.gov/ssc/data/access/lat/BackgroundModels.html 99 http://project-mathlibs.web.cern.ch/project-mathlibs/sw/5_15_04/ Minuit2/html
} 
Table 9

Spectral Fitting Results for Young LAT-detected Pulsars

\begin{tabular}{|c|c|c|c|c|c|c|c|c|c|}
\hline $\mathrm{PSR}^{\mathrm{a}}$ & $\begin{array}{l}\text { Photon Flux } \\
\left(\mathrm{ph} \mathrm{cm}^{-2} \mathrm{~s}^{-1}\right) \\
\left(\times 10^{-8}\right)\end{array}$ & $\begin{array}{l}\text { Energy Flux } \\
\left(\operatorname{erg~cm}^{-2} \mathrm{~s}^{-1}\right) \\
\quad\left(\times 10^{-11}\right)\end{array}$ & $\Gamma$ & $\begin{array}{c}E_{\text {cut }} \\
(\mathrm{GeV})\end{array}$ & TS & $\mathrm{TS}_{\text {cut }}$ & $\mathrm{TS}_{\mathrm{b} \text { free }}$ & $\begin{array}{l}\text { Luminosity } \\
\left(10^{33} \mathrm{erg} \mathrm{s}^{-1}\right)\end{array}$ & $\begin{array}{c}\text { Efficiency }^{\mathrm{b}} \\
(\%)\end{array}$ \\
\hline $\mathrm{J} 0007+7303$ & $323 \pm 0.4$ & $40.1 \pm 0.4$ & $1.4 \pm 0.1$ & $4.7 \pm 0.2$ & 43388 & 1884 & 4 & $94 \pm 1 \pm 40$ & $21.0 \pm 0.2 \pm 8$ \\
\hline J0106+4855 & $1.7 \pm 0.4$ & $1.9 \pm 0.2$ & $1.2 \pm 0.2$ & $2.7 \pm 0.6$ & 544 & 58 & 2 & $21 \pm 2_{-8}^{+20}$ & $71 \pm 7_{-30}^{+60}$ \\
\hline $\mathrm{J} 0205+6449$ & $10.5 \pm 0.7$ & $5.4 \pm 0.2$ & $1.8 \pm 0.1$ & $1.6 \pm 0.3$ & 1019 & 86 & 7 & $24 \pm 1 \pm 1.0$ & $0.09 \pm 0.01 \pm 0.01$ \\
\hline $\mathrm{J} 0248+6021$ & $9.9 \pm 1.3$ & $5.2 \pm 0.4$ & $1.8 \pm 0.1$ & $1.6 \pm 0.3$ & 578 & 61 & 0 & $25 \pm 2 \pm 5$ & $12 \pm 1 \pm 2$ \\
\hline $\mathrm{J} 0357+3205$ & $9.0 \pm 0.4$ & $6.4 \pm 0.2$ & $1.0 \pm 0.1$ & $0.8 \pm 0.1$ & 3468 & 461 & 2 & $\ldots$ & $\ldots$ \\
\hline $\mathrm{J} 0534+2200$ & $208 \pm 1$ & $129.3 \pm 0.8$ & $1.9 \pm 0.1$ & $4.2 \pm 0.2$ & 102653 & 1461 & 13 & $619 \pm 4 \pm 300$ & $0.14 \pm 0.01 \pm 0.1$ \\
\hline $\mathrm{J} 0622+3749$ & $2.0 \pm 0.3$ & $1.4 \pm 0.1$ & $0.6 \pm 0.4$ & $0.6 \pm 0.1$ & 302 & 91 & 0 & $\ldots$ & $\ldots$ \\
\hline $\mathrm{J} 0631+1036$ & $6.4 \pm 0.6$ & $4.7 \pm 0.3$ & $1.8 \pm 0.1$ & $6 \pm 1$ & 621 & 39 & 1 & $5.6 \pm 0.3_{-2}^{+3}$ & $3.2 \pm 0.2_{-1}^{+2}$ \\
\hline J0633+0632 & $9.7 \pm 1.1$ & $9.4 \pm 0.5$ & $1.4 \pm 0.1$ & $2.7 \pm 0.3$ & 2448 & 203 & 9 & $\ldots$ & $\ldots$ \\
\hline J0633+1746 & $416 \pm 1$ & $423.3 \pm 1.2$ & $1.2 \pm 0.1$ & $2.2 \pm 0.1$ & 906994 & 33861 & 277 & $31.7 \pm 0.1_{-20}^{+90}$ & $97.4 \pm 0.3_{-50}^{+300}$ \\
\hline J0659+1414 & $7.1 \pm 0.6$ & $2.5 \pm 0.2$ & $1.7 \pm 0.5$ & $0.4 \pm 0.2$ & 419 & 33 & 0 & $0.24 \pm 0.02 \pm 0.05$ & $0.62 \pm 0.04 \pm 0.1$ \\
\hline J0729-1448† & $\ldots$ & $\ldots$ & $\ldots$ & $\ldots$ & 54 & 26 & 0 & $\ldots$ & $\ldots$ \\
\hline J0734-1559 & $10.8 \pm 0.7$ & $5.6 \pm 0.2$ & $2.0 \pm 0.1$ & $3.2 \pm 0.9$ & 916 & 39 & 9 & $\ldots$ & $\ldots$ \\
\hline $\mathrm{J} 0742-2822$ & $3.2 \pm 0.6$ & $1.7 \pm 0.2$ & $1.7 \pm 0.3$ & $1.6 \pm 0.8$ & 112 & 11 & 2 & $9 \pm 1 \pm 4$ & $6.2 \pm 0.7 \pm 3$ \\
\hline J0835-4510 & $1088 \pm 2$ & $906 \pm 2$ & $1.5 \pm 0.1$ & $3.0 \pm 0.1$ & 1659005 & 43084 & 916 & $89.3 \pm 0.2 \pm 10$ & $1.3 \pm 0.1 \pm 0.1$ \\
\hline J0908-4913 & $7.9 \pm 1.3$ & $4.4 \pm 0.4$ & $1.0 \pm 0.4$ & $0.5 \pm 0.2$ & 315 & 82 & 0 & $35 \pm 3_{-20}^{+30}$ & $7.1 \pm 0.7_{-4}^{+6}$ \\
\hline J0940-5428† & $\ldots$ & $\ldots$ & $\ldots$ & $\ldots$ & 14 & 13 & 8 & $\ldots$ & $\ldots$ \\
\hline $\mathrm{J} 1016-5857$ & $6.9 \pm 2.4$ & $5.4 \pm 0.9$ & $1.8 \pm 0.2$ & $6 \pm 3$ & 290 & 13 & 0 & $55 \pm 9_{-50}^{+30}$ & $2.1 \pm 0.4_{-2}^{+1}$ \\
\hline $\mathrm{J} 1019-5749 \dagger$ & $\ldots$ & $\ldots$ & $\ldots$ & $\ldots$ & 21 & 0 & 0 & $\ldots$ & $\ldots$ \\
\hline $\mathrm{J} 1023-5746$ & $30 \pm 3$ & $19.5 \pm 1.2$ & $1.7 \pm 0.1$ & $2.5 \pm 0.4$ & 2926 & 162 & 20 & $\ldots$ & $\ldots$ \\
\hline J1028-5819 & $31 \pm 2$ & $24.3 \pm 0.8$ & $1.7 \pm 0.1$ & $4.6 \pm 0.5$ & 5096 & 235 & 28 & $158 \pm 5 \pm 40$ & $18.9 \pm 0.6 \pm 5$ \\
\hline J1044-5737 & $26 \pm 1$ & $15.6 \pm 0.5$ & $1.8 \pm 0.1$ & $2.8 \pm 0.3$ & 3380 & 202 & 19 & $\ldots$ & $\ldots$ \\
\hline J1048-5832 & $25 \pm 2$ & $19.6 \pm 0.6$ & $1.6 \pm 0.1$ & $3.0 \pm 0.3$ & 5389 & 325 & 30 & $176 \pm 5 \pm 40$ & $8.8 \pm 0.3 \pm 2$ \\
\hline $\mathrm{J} 1057-5226$ & $32 \pm 1$ & $29.5 \pm 0.3$ & $1.0 \pm 0.1$ & $1.4 \pm 0.1$ & 27848 & 2377 & 5 & $4.3 \pm 0.1_{-3}^{+5}$ & $14.4 \pm 0.2^{ \pm} 10$ \\
\hline $\mathrm{J} 1105-6107$ & $7.8 \pm 1.6$ & $4.9 \pm 0.6$ & $1.5 \pm 0.3$ & $1.3 \pm 0.6$ & 309 & 42 & 8 & $150 \pm 20 \pm 50$ & $5.9 \pm 0.7 \pm 2$ \\
\hline $\mathrm{J} 1112-6103$ & $1.9 \pm 0.9$ & $2.0 \pm 0.5$ & $1.6 \pm 0.3$ & $6 \pm 3$ & 58 & 6 & 0 & $360 \pm 90_{-200}^{+600}$ & $8 \pm 2_{-4}^{+10}$ \\
\hline J1119-6127 & $11 \pm 2$ & $7.1 \pm 0.5$ & $1.8 \pm 0.1$ & $3.2 \pm 0.8$ & 661 & 37 & 13 & $600 \pm 40 \pm 60$ & $26 \pm 2 \pm 2$ \\
\hline J1124-5916 & $10 \pm 1$ & $6.2 \pm 0.4$ & $1.8 \pm 0.1$ & $2.1 \pm 0.4$ & 1058 & 79 & 6 & $170 \pm 10_{-70}^{+50}$ & $1.4 \pm 0.1_{-0.6}^{+0.4}$ \\
\hline $\mathrm{J} 1135-6055$ & $7.4 \pm 0.9$ & $4.8 \pm 0.3$ & $1.7 \pm 0.1$ & $2.4 \pm 0.5$ & 498 & 61 & 3 & $\ldots$ & $\ldots$ \\
\hline J1357-6429 & $7.8 \pm 1.1$ & $3.4 \pm 0.3$ & $1.8 \pm 0.4$ & $0.9 \pm 0.5$ & 187 & 20 & 0 & $25 \pm 2_{-8}^{+10}$ & $0.82 \pm 0.08_{-0.3}^{+0.4}$ \\
\hline $\mathrm{J} 1410-6132 \dagger$ & $3 \pm 3$ & $3 \pm 1$ & $\ldots$ & $\ldots$ & 40 & 9 & 0 & $800 \pm 300_{-400}^{+900}$ & $8 \pm 3_{-4}^{+9}$ \\
\hline $\mathrm{J} 1413-6205$ & $16 \pm 2$ & $15.7 \pm 0.6$ & $1.5 \pm 0.1$ & $4.1 \pm 0.5$ & 1795 & 180 & 1 & $\ldots$ & $\ldots$ \\
\hline $\mathrm{J} 1418-6058$ & $38 \pm 3$ & $30.2 \pm 1.4$ & $1.8 \pm 0.1$ & $5.5 \pm 0.5$ & 3487 & 172 & 1 & $92 \pm 4_{-60}^{+100}$ & $1.9 \pm 0.1_{-1}^{+2}$ \\
\hline J1420-6048 & $26 \pm 3$ & $17.0 \pm 1.4$ & $1.5 \pm 0.1$ & $1.6 \pm 0.2$ & 1220 & 51 & 2 & $640 \pm 50 \pm 200$ & $6.2 \pm 0.5 \pm 2$ \\
\hline J1429-5911 & $12 \pm 1$ & $8.0 \pm 0.4$ & $1.6 \pm 0.1$ & $2.2 \pm 0.3$ & 822 & 124 & 0 & $\ldots$ & $\ldots$ \\
\hline $\mathrm{J} 1459-6053$ & $24 \pm 2$ & $12.9 \pm 0.4$ & $2.0 \pm 0.1$ & $2.9 \pm 0.5$ & 2046 & 103 & 15 & $\ldots$ & $\ldots$ \\
\hline J1509-5850 & $20 \pm 2$ & $12.7 \pm 0.7$ & $1.9 \pm 0.1$ & $4.6 \pm 0.9$ & 1152 & 67 & 0 & $105 \pm 6 \pm 40$ & $20 \pm 1 \pm 7$ \\
\hline $\mathrm{J} 1513-5908 \dagger$ & $10 \pm 2$ & $3.2 \pm 0.7$ & $\ldots$ & $\ldots$ & 98 & 5 & 1 & $70 \pm 10 \pm 20$ & $0.4 \pm 0.1 \pm 0.1$ \\
\hline $\mathrm{J} 1531-5610 \dagger \star$ & $0.1 \pm 0.05$ & $0.2 \pm 0.1$ & $\ldots$ & $\ldots$ & 2 & 2 & 1 & $1.0 \pm 0.6 \pm 0.4$ & $0.11 \pm 0.07 \pm 0.04$ \\
\hline J1620-4927 & $15 \pm 2$ & $15.7 \pm 0.8$ & $1.3 \pm 0.1$ & $2.5 \pm 0.3$ & 1407 & 199 & 4 & $\ldots$ & $\ldots$ \\
\hline J1648-4611 & $5.3 \pm 1.6$ & $5.4 \pm 0.8$ & $1.6 \pm 0.3$ & $6 \pm 4$ & 176 & 17 & 0 & $160 \pm 20 \pm 40$ & $80 \pm 10 \pm 20$ \\
\hline $\mathrm{J} 1702-4128 \star$ & $4.4 \pm 5.2$ & $2.8 \pm 2.7$ & $1.1 \pm 0.9$ & $0.8 \pm 0.5$ & 62 & 7 & 0 & $80 \pm 70 \pm 20$ & $20 \pm 20 \pm 5$ \\
\hline J1709-4429 & $160 \pm 2$ & $135 \pm 1$ & $1.6 \pm 0.1$ & $4.2 \pm 0.1$ & 96893 & 3433 & 132 & $853 \pm 6 \pm 200$ & $25.1 \pm 0.2 \pm 5$ \\
\hline $\mathrm{J} 1718-3825$ & $14 \pm 1$ & $8.9 \pm 0.5$ & $1.5 \pm 0.1$ & $1.4 \pm 0.1$ & 462 & 81 & 1 & $138 \pm 8 \pm 30$ & $11.0 \pm 0.6 \pm 3$ \\
\hline $\mathrm{J} 1730-3350 \star$ & $3.4 \pm 0.1$ & $2.4 \pm 0.4$ & $1.5 \pm 0.3$ & $1.2 \pm 0.3$ & 100 & 21 & 1 & $36 \pm 6 \pm 9$ & $2.9 \pm 0.5 \pm 0.7$ \\
\hline J1732-3131 & $17 \pm 1$ & $19.4 \pm 0.6$ & $1.0 \pm 0.1$ & $1.9 \pm 0.1$ & 2821 & 550 & 0 & $8.6 \pm 0.3 \pm 2$ & $5.9 \pm 0.2 \pm 1$ \\
\hline J1741-2054 & $17 \pm 1$ & $11.7 \pm 0.4$ & $1.1 \pm 0.1$ & $0.9 \pm 0.1$ & 3014 & 464 & 0 & $2.1 \pm 0.1 \pm 0.2$ & $22 \pm 1 \pm 3$ \\
\hline J1746-3239 & $10 \pm 1$ & $7.2 \pm 0.5$ & $1.4 \pm 0.1$ & $1.5 \pm 0.2$ & 654 & 109 & 0 & $\ldots$ & $\ldots$ \\
\hline J1747-2958 & $33 \pm 3$ & $21.1 \pm 1.0$ & $1.6 \pm 0.1$ & $1.9 \pm 0.1$ & 1689 & 211 & 2 & $570 \pm 30 \pm 200$ & $23 \pm 1 \pm 7$ \\
\hline $\mathrm{J} 1801-2451 \star$ & $1.4 \pm 1.0$ & $1.2 \pm 0.5$ & $1.5 \pm 0.5$ & $3 \pm 2$ & 58 & 10 & 6 & $40 \pm 10_{-7}^{+9}$ & $1.5 \pm 0.6_{-0.3}^{+0.4}$ \\
\hline J1803-2149 & $11 \pm 2$ & $9.2 \pm 0.8$ & $1.6 \pm 0.1$ & $3.6 \pm 0.8$ & 410 & 40 & 1 & $\ldots$ & $\ldots$ \\
\hline J1809-2332 & $60 \pm 2$ & $47.4 \pm 0.8$ & $1.6 \pm 0.1$ & $3.4 \pm 0.2$ & 15781 & 901 & 41 & $164 \pm 3_{-100}^{+200}$ & $38 \pm 1_{-30}^{+60}$ \\
\hline J1813-1246 & $45 \pm 2$ & $25.3 \pm 0.6$ & $1.9 \pm 0.1$ & $2.6 \pm 0.3$ & 4664 & 272 & 1 & $\ldots$ & $\ldots$ \\
\hline J1826-1256 & $54 \pm 3$ & $38.3 \pm 0.9$ & $1.6 \pm 0.1$ & $2.2 \pm 0.2$ & 5160 & 533 & 23 & $\ldots$ & $\ldots$ \\
\hline J1833-1034 & $7.0 \pm 1.1$ & $5.9 \pm 0.5$ & $0.9 \pm 0.2$ & $0.9 \pm 0.2$ & 258 & 78 & 0 & $160 \pm 10 \pm 30$ & $0.46 \pm 0.04 \pm 0.08$ \\
\hline $\mathrm{J} 1835-1106 \dagger \star$ & $0.4 \pm 0.1$ & $0.6 \pm 0.2$ & $\ldots$ & $\ldots$ & 30 & 18 & 0 & $6 \pm 2 \pm 2$ & $3 \pm 1 \pm 0.9$ \\
\hline $\mathrm{J} 1836+5925$ & $63 \pm 1$ & $60.6 \pm 0.4$ & $1.2 \pm 0.1$ & $2.0 \pm 0.1$ & 142427 & 5747 & 28 & $20.4 \pm 0.1_{-20}^{+30}$ & $180 \pm 1_{-100}^{+200}$ \\
\hline J1838-0537 & $22 \pm 2$ & $18.8 \pm 0.9$ & $1.6 \pm 0.1$ & $4.1 \pm 0.4$ & 1325 & 114 & 1 & $\ldots$ & $\ldots$ \\
\hline J1846+0919 & $1.4 \pm 0.3$ & $2.4 \pm 0.2$ & $0.7 \pm 0.3$ & $2.2 \pm 0.5$ & 428 & 79 & 0 & $\ldots$ & $\ldots$ \\
\hline $\mathrm{J} 1907+0602$ & $34 \pm 2$ & $25.4 \pm 0.6$ & $1.6 \pm 0.1$ & $2.9 \pm 0.3$ & 3773 & 390 & 35 & $314 \pm 8 \pm 60$ & $11.1 \pm 0.3 \pm 2$ \\
\hline $\mathrm{J} 1952+3252$ & $17 \pm 1$ & $13.8 \pm 0.3$ & $1.5 \pm 0.1$ & $2.5 \pm 0.2$ & 4469 & 365 & 11 & $66 \pm 2_{-30}^{+40}$ & $1.8 \pm 0.1_{-0.8}^{+1.0}$ \\
\hline J1954+2836 & $13 \pm 1$ & $10.3 \pm 0.4$ & $1.6 \pm 0.1$ & $3.3 \pm 0.4$ & 1592 & 168 & 11 & $\ldots$ & $\ldots$ \\
\hline $\mathrm{J} 1957+5033$ & $4.0 \pm 0.4$ & $2.6 \pm 0.1$ & $1.3 \pm 0.2$ & $1.0 \pm 0.2$ & 846 & 105 & 0 & $\ldots$ & $\ldots$ \\
\hline
\end{tabular}


Table 9

(Continued)

\begin{tabular}{|c|c|c|c|c|c|c|c|c|c|}
\hline $\mathrm{PSR}^{\mathrm{a}}$ & $\begin{array}{l}\text { Photon Flux } \\
\left(\mathrm{ph} \mathrm{cm}^{-2} \mathrm{~s}^{-1}\right) \\
\left(\times 10^{-8}\right)\end{array}$ & $\begin{array}{c}\text { Energy Flux } \\
\left(\mathrm{erg} \mathrm{cm}^{-2} \mathrm{~s}^{-1}\right) \\
\left(\times 10^{-11}\right)\end{array}$ & $\Gamma$ & $\begin{array}{c}E_{\text {cut }} \\
(\mathrm{GeV})\end{array}$ & TS & $\mathrm{TS}_{\text {cut }}$ & $\mathrm{TS}_{\mathrm{b} \text { free }}$ & $\begin{array}{l}\text { Luminosity } \\
\left(10^{33} \mathrm{erg} \mathrm{s}^{-1}\right)\end{array}$ & $\begin{array}{c}\text { Efficiency }^{\mathrm{b}} \\
(\%)\end{array}$ \\
\hline $\mathrm{J} 1958+2846$ & $11 \pm 1$ & $9.1 \pm 0.4$ & $1.4 \pm 0.1$ & $2.0 \pm 0.3$ & 1519 & 206 & 25 & $\ldots$ & $\ldots$ \\
\hline $\mathrm{J} 2021+3651$ & $68 \pm 2$ & $49.4 \pm 0.8$ & $1.7 \pm 0.1$ & $3.0 \pm 0.2$ & 17821 & 998 & 23 & $5910 \pm 90_{-4000}^{+3000}$ & $175 \pm 3_{-100}^{+80}$ \\
\hline $\mathrm{J} 2021+4026$ & $138 \pm 2$ & $95.5 \pm 0.9$ & $1.6 \pm 0.1$ & $2.6 \pm 0.1$ & 53955 & 2343 & 72 & $257 \pm 2_{-100}^{+200}$ & $225 \pm 2_{-100}^{+200}$ \\
\hline J2028+3332 & $5.8 \pm 0.9$ & $5.8 \pm 0.4$ & $1.2 \pm 0.2$ & $1.9 \pm 0.3$ & 1058 & 161 & 0 & $\ldots$ & $\ldots$ \\
\hline $\mathrm{J} 2030+3641$ & $2.3 \pm 0.6$ & $3.1 \pm 0.3$ & $0.7 \pm 0.4$ & $1.5 \pm 0.4$ & 313 & 91 & 0 & $34 \pm 4_{-20}^{+30}$ & $110 \pm 10_{-60}^{+80}$ \\
\hline $\mathrm{J} 2030+4415$ & $9.3 \pm 1.0$ & $5.8 \pm 0.4$ & $1.6 \pm 0.1$ & $1.7 \pm 0.3$ & 504 & 74 & 0 & $\ldots$ & $\ldots$ \\
\hline $\mathrm{J} 2032+4127$ & $7.4 \pm 1.1$ & $10.6 \pm 0.6$ & $1.1 \pm 0.1$ & $3.2 \pm 0.5$ & 1383 & 162 & 0 & $169 \pm 10 \pm 50$ & $62 \pm 4 \pm 20$ \\
\hline $\mathrm{J} 2043+2740$ & $1.5 \pm 0.4$ & $1.0 \pm 0.1$ & $1.4 \pm 0.4$ & $1.2 \pm 0.6$ & 97 & 18 & 2 & $3.8 \pm 0.6 \pm 1$ & $7 \pm 1 \pm 2$ \\
\hline $\mathrm{J} 2055+2539$ & $6.2 \pm 0.4$ & $5.4 \pm 0.2$ & $1.0 \pm 0.1$ & $1.1 \pm 0.1$ & 2751 & 361 & 1 & $\ldots$ & $\ldots$ \\
\hline $\mathrm{J} 2111+4606$ & $5.3 \pm 0.7$ & $4.4 \pm 0.3$ & $1.7 \pm 0.1$ & $5 \pm 1$ & 731 & 45 & 0 & $\ldots$ & $\ldots$ \\
\hline $\mathrm{J} 2139+4716$ & $3.1 \pm 0.5$ & $2.3 \pm 0.2$ & $1.3 \pm 0.2$ & $1.3 \pm 0.3$ & 369 & 71 & 2 & $\ldots$ & $\ldots$ \\
\hline $\mathrm{J} 2229+6114$ & $38 \pm 1$ & $25.3 \pm 0.4$ & $1.8 \pm 0.1$ & $4.3 \pm 0.3$ & 12101 & 424 & 54 & $19.4 \pm 0.3 \pm 8$ & $0.09 \pm 0.01 \pm 0.04$ \\
\hline $\mathrm{J} 2238+5903$ & $9.3 \pm 0.9$ & $6.4 \pm 0.3$ & $1.6 \pm 0.1$ & $2.1 \pm 0.3$ & 1165 & 123 & 10 & $\ldots$ & $\ldots$ \\
\hline $\mathrm{J} 2240+5832$ & $1.1 \pm 0.7$ & $1.1 \pm 0.3$ & $1.5 \pm 0.5$ & $3 \pm 2$ & 54 & 11 & 0 & $80 \pm 20 \pm 10$ & $40 \pm 10 \pm 6$ \\
\hline
\end{tabular}

Notes. Unbinned maximum likelihood spectral fit results for the young LAT gamma-ray pulsars, using the PLEC1 model (Equation (12) in Section 6). Columns 2 and 3 list the phase-averaged integral photon and energy fluxes in the 0.1 to $100 \mathrm{GeV}$ energy band, $F_{100}$ and $G_{100}$. Columns 4 and 5 list the photon index $\Gamma$ and cutoff energy $E_{\text {cut }}$. Columns 6, 7, and 8 list the source significance TS, significance $\mathrm{TS}_{\text {cut }}$ of the exponential cutoff compared to a simple power law, and significance $\mathrm{TS}_{\mathrm{b}}$ free of the PLEC compared to a PLEC1 shape. A value $\mathrm{TS}_{\text {cut }}<9$ indicates that a spectral cutoff is not significantly detected. Columns 9 and 10 give the total gamma-ray luminosity $L_{\gamma}$ in the 0.1 to $100 \mathrm{GeV}$ energy band, and the gamma-ray conversion efficiency $\eta \equiv L_{\gamma} / \dot{E}$, assuming $f_{\Omega}=1$ as described in Section 6.3 . The first uncertainty in $L_{\gamma}$ and $\eta$ comes from the statistical uncertainties in the spectral fit, whereas the second is due to the distance uncertainty. The strong dependence of these quantities on distance (see Table 5) and beaming factor means that these values should be considered with care.

a A dagger $(\dagger)$ means the spectral fit is unreliable (see text). A star $(\star)$ means that the spectrum was calculated using the on-peak data only.

${ }^{\mathrm{b}}$ Overestimated distances or the assumed beaming factor, $f_{\Omega}=1$, can result in an efficiency $>100 \%$.

Statistical uncertainties on $F_{100}$ and $G_{100}$ are obtained using derivatives with respect to the primary parameters and the covariance matrix obtained from the fitting process.

We tested the validity of modeling the pulsar spectrum as a power law with a simple exponential cutoff shape (PLEC1; Equation (12) with $b \equiv 1$ ) by repeating the analysis using a pure power-law shape (PL), and a power law with a more general exponential cutoff shape leaving the $b$ parameter free (PLEC). For a number of pulsars, a PLEC1 spectral model is not significantly better than a PL. We identified these by computing $\mathrm{TS}_{\text {cut }} \equiv 2 \Delta \log$ (likelihood) (comparable to a $\chi^{2}$ distribution with one degree of freedom) between the models with and without the cutoff. We say that the PLEC1 model is not significantly preferred over the PL model for pulsars with $\mathrm{TS}_{\text {cut }}<9$, listed in Tables 9 and 10. Similarly, we calculated $\mathrm{TS}_{\mathrm{b} \text { free }} \equiv 2 \Delta \log$ (likelihood) between the PLEC1 and PLEC models, also listed in the tables.

In all cases where PLEC is significantly preferred over the PLEC1 model $\left(\mathrm{TS}_{\mathrm{b} \text { free }} \geqslant 9\right)$, the maximum likelihood value of $b$ is significantly less than 1 , indicating a sub-exponential cutoff. As noted by Abdo et al. (2010n) and Celik \& Johnson (2011), a sub-exponential cutoff is a functional form which approximates the superposition of several PLEC1 models with varying values of $\Gamma$ and $E_{\text {cut }}$ as different regions of the pulsar magnetosphere cut across our line of sight. This is further supported by analysis of PSR J1057-5226 for which the $b$ parameter in a PLEC fit is consistent with 1 and the spectral parameters show very little variation with phase (Abdo et al. 2010o). Thus, no physical quantities can be derived from the PLEC best-fit parameters, whereas the PLEC1 best-fit parameters can be used if taken as flux-weighted, average measures of $E_{\text {cut }}$ and $\Gamma$. Further, in our PLEC fits, the $E_{\text {cut }}$ value was often at the minimum boundary $(0.1 \mathrm{GeV})$ with unrealistically small uncertainties. Therefore, we do not report the PLEC fit values in the tables and instead indicate pulsars with high $\mathrm{TS}_{\mathrm{b} \text { free }}$ as interesting candidates for phase-resolved spectral analysis, a task beyond the scope of this catalog. For pulsars with $\mathrm{TS}_{\mathrm{b}}$ free $\geqslant 9$, the PLEC fit results are included in the spectral plots (see the examples amongst Figures 23(a)-(i) in Appendix A) and in the auxiliary files (Appendix B). We encourage comparing predicted spectra from different emission models to the energy sub-band fluxes, which are also included in the auxiliary files. In some pulsars, particularly the Crab, a preference for $b<1$ may indicate either the presence of a secondary spectral component that dominates above $\gtrsim 10 \mathrm{GeV}$ or that the curvature radiation assumption is incorrect (as argued by Lyutikov et al. 2012, for example). Such a determination is difficult using the LAT data alone (see Section 8.4).

For some pulsars with low duty cycles, the phase-averaged analysis returned a low TS value and/or could not constrain the spectral parameters well. We selected the off-peak intervals for these sources and followed the same prescription as for the phase-averaged analyses but without the pulsar in the model. We then fixed the parameters of all sources $>4^{\circ}$ from the pulsar, left the normalization parameters of the remaining point sources and diffuse models free, added the pulsar back to the model, and performed an on-peak spectral analysis. These pulsars are indicated by a star $(\star)$ in Tables 9 and 10 . The values of $F_{100}$ and $G_{100}$ reported for these pulsars have been corrected to phase-averaged values. Additionally, the spatial residuals for PSR J1702-4128 revealed a large deficit near the pulsar attributed to the Galactic diffuse model. In lieu of a new Galactic diffuse template, we increased the minimum event energy to $300 \mathrm{MeV}$ and performed an on-peak spectral analysis as described previously. Thus, for PSR J1702-4128 the reported values of $F_{100}$ and $G_{100}$ are extrapolations below the energy range of the data, which increases the quoted uncertainties beyond the statistical values. 
Table 10

Spectral Fitting Results for LAT-detected Millisecond Pulsars

\begin{tabular}{|c|c|c|c|c|c|c|c|c|c|}
\hline $\mathrm{PSR}^{\mathrm{a}}$ & $\begin{array}{c}\text { Photon Flux } \\
\left(\mathrm{ph} \mathrm{cm}^{-2} \mathrm{~s}^{-1}\right) \\
\left(\times 10^{-8}\right)\end{array}$ & $\begin{array}{c}\text { Energy Flux } \\
\left(\mathrm{erg} \mathrm{cm}^{-2} \mathrm{~s}^{-1}\right) \\
\left(\times 10^{-11}\right)\end{array}$ & $\Gamma$ & $\begin{array}{c}E_{\text {cut }} \\
(\mathrm{GeV})\end{array}$ & TS & $\mathrm{TS}_{\text {cut }}$ & $\mathrm{TS}_{\mathrm{b} \text { free }}$ & $\begin{array}{l}\text { Luminosity } \\
\left(10^{32} \mathrm{erg} \mathrm{s}^{-1}\right)\end{array}$ & $\begin{array}{c}\text { Efficiency }{ }^{\mathrm{b}} \\
(\%)\end{array}$ \\
\hline J0023+0923 & $1.2 \pm 0.4$ & $0.80 \pm 0.12$ & $1.4 \pm 0.4$ & $1.4 \pm 0.6$ & 131 & 16 & 7 & $4.6 \pm 0.7_{-1}^{+3}$ & $3.0 \pm 0.5_{-1}^{+2}$ \\
\hline J0030+0451 & $6.6 \pm 0.3$ & $6.14 \pm 0.18$ & $1.2 \pm 0.1$ & $1.8 \pm 0.2$ & 4788 & 316 & 10 & $5.8 \pm 0.2_{-2}^{+5}$ & $16 \pm 1_{-6}^{+13}$ \\
\hline J0034-0534 & $2.2 \pm 0.3$ & $1.62 \pm 0.12$ & $1.4 \pm 0.2$ & $1.8 \pm 0.4$ & 563 & 45 & 0 & $5.7 \pm 0.4_{-2}^{+3}$ & $3.3 \pm 0.2_{-1.1}^{+1.5}$ \\
\hline J0101-6422 & $0.75 \pm 0.14$ & $1.05 \pm 0.09$ & $0.7 \pm 0.3$ & $1.5 \pm 0.4$ & 491 & 59 & 1 & $3.8 \pm 0.3 \pm 1$ & $3.8 \pm 0.3_{-1.0}^{+1.1 .3}$ \\
\hline $\mathrm{J} 0102+4839$ & $1.3 \pm 0.3$ & $1.32 \pm 0.16$ & $1.4 \pm 0.3$ & $3.2 \pm 1.1$ & 251 & 29 & 1 & $90 \pm 10_{-30}^{+40}$ & $49 \pm 6_{-16}^{+23}$ \\
\hline $\mathrm{J} 0218+4232$ & $7.7 \pm 0.7$ & $4.56 \pm 0.24$ & $2.0 \pm 0.1$ & $4.6 \pm 1.2$ & 1313 & 38 & 1 & $380 \pm 20_{-200}^{+400}$ & $16 \pm 1_{-7}^{+15}$ \\
\hline $\mathrm{J} 0340+4130$ & $1.5 \pm 0.2$ & $2.04 \pm 0.15$ & $1.1 \pm 0.2$ & $2.6 \pm 0.6$ & 553 & 73 & 4 & $73 \pm 6 \pm 20$ & $92.6 \pm 7.0 \pm 29$ \\
\hline J0437-4715 & $2.7 \pm 0.3$ & $1.67 \pm 0.11$ & $1.4 \pm 0.2$ & $1.1 \pm 0.3$ & 687 & 67 & 1 & $0.49 \pm 0.03 \pm 0.01$ & $1.7 \pm 0.1 \pm 0.1$ \\
\hline J0610-2100 & $0.78 \pm 0.25$ & $0.66 \pm 0.11$ & $1.2 \pm 0.4$ & $1.6 \pm 0.8$ & 98 & 14 & 1 & $100 \pm 20_{-50}^{+500}$ & $1180 \pm 190_{-570}^{+6400}$ \\
\hline J0613-0200 & $2.7 \pm 0.4$ & $2.99 \pm 0.19$ & $1.2 \pm 0.2$ & $2.5 \pm 0.5$ & 760 & 88 & 0 & $29 \pm 2_{-10}^{+30}$ & $24.1 \pm 1.5_{-9.5}^{+26}$ \\
\hline J0614-3329 & $8.5 \pm 0.3$ & $10.94 \pm 0.27$ & $1.3 \pm 0.1$ & $3.9 \pm 0.3$ & 9408 & 416 & 11 & $470 \pm 10 \pm 200$ & $215 \pm 5_{-72}^{+110}$ \\
\hline J0751+1807 & $1.1 \pm 0.2$ & $1.33 \pm 0.12$ & $1.1 \pm 0.2$ & $2.6 \pm 0.7$ & 427 & 46 & 1 & $2.5 \pm 0.2_{-1}^{+3}$ & $3.5 \pm 0.3_{-1.5}^{+4.4}$ \\
\hline $\mathrm{J} 1024-0719 \dagger$ & $0.2 \pm 0.2$ & $0.3 \pm 0.1$ & & & 46 & 11 & 0 & $0.6 \pm 0.2 \pm 0.1$ & $12 \pm 4 \pm 2.3$ \\
\hline J1124-3653 & $0.94 \pm 0.23$ & $1.21 \pm 0.13$ & $1.1 \pm 0.3$ & $2.5 \pm 0.7$ & 293 & 39 & 0 & $43 \pm 5 \pm 20$ & $25.0 \pm 2.7_{-9.4}^{+14}$ \\
\hline J1125-5825 & $1.1 \pm 0.5$ & $0.89 \pm 0.20$ & $1.7 \pm 0.2$ & $4.8 \pm 2.4$ & 41 & 4 & 1 & $70 \pm 20^{ \pm} 20$ & $9.1 \pm 2.0_{-2.4}^{+3.0}$ \\
\hline $\mathrm{J} 1231-1411$ & $9.2 \pm 0.4$ & $10.28 \pm 0.26$ & $1.2 \pm 0.1$ & $2.7 \pm 0.2$ & 7931 & 446 & 3 & $24 \pm 0.6 \pm 5$ & $45.9 \pm 1.2 \pm 9.9$ \\
\hline J1446-4701 & $0.73 \pm 0.31$ & $0.74 \pm 0.14$ & $1.4 \pm 0.4$ & $3.0 \pm 1.7$ & 71 & 9 & 0 & $19 \pm 4 \pm 5$ & $5.2 \pm 1.0 \pm 1.4$ \\
\hline J1514-4946 & $4.1 \pm 0.6$ & $4.50 \pm 0.28$ & $1.5 \pm 0.1$ & $5.3 \pm 1.1$ & 999 & 66 & 0 & $48 \pm 3 \pm 10$ & $29.7 \pm 1.8 \pm 7.1$ \\
\hline $\mathrm{J} 1600-3053 \star$ & $0.22 \pm 0.16$ & $0.53 \pm 0.28$ & $0.40 \pm 0.47$ & $2.0 \pm 0.7$ & 147 & 26 & 0 & $17 \pm 9_{-5}^{+7}$ & $23 \pm 12_{-7.0}^{+9.6}$ \\
\hline $\mathrm{J} 1614-2230$ & $2.0 \pm 0.4$ & $2.44 \pm 0.20$ & $0.96 \pm 0.22$ & $1.9 \pm 0.4$ & 599 & 81 & 1 & $12 \pm 1 \pm 2$ & $32.6 \pm 2.6 \pm 4.8$ \\
\hline J1658-5324 & $5.7 \pm 0.7$ & $2.89 \pm 0.23$ & $1.8 \pm 0.2$ & $1.4 \pm 0.4$ & 327 & 34 & 0 & $30 \pm 2 \pm 8$ & $9.9 \pm 0.8 \pm 2.6$ \\
\hline $\mathrm{J} 1713+0747$ & $1.3 \pm 0.4$ & $1.02 \pm 0.14$ & $1.6 \pm 0.3$ & $2.7 \pm 1.2$ & 126 & 17 & 2 & $13 \pm 2_{-1}^{+2}$ & $39.0 \pm 5.6_{-3.6}^{+4.6}$ \\
\hline $\mathrm{J} 1741+1351 \dagger$ & $0.12 \pm 0.04$ & $0.24 \pm 0.08$ & $\ldots$ & & 23 & 7 & 0 & $3 \pm 1 \pm 0.3$ & $1.5 \pm 0.5 \pm 0.1$ \\
\hline $\mathrm{J} 1744-1134$ & $4.6 \pm 0.7$ & $3.25 \pm 0.25$ & $1.3 \pm 0.2$ & $1.2 \pm 0.3$ & 394 & 65 & 0 & $6.8 \pm 0.5 \pm 0.5$ & $16.5 \pm 1.3 \pm 1.3$ \\
\hline J1747-4036 & $1.5 \pm 0.7$ & $0.99 \pm 0.23$ & $1.9 \pm 0.3$ & $5.4 \pm 3.3$ & 43 & 4 & 0 & $140 \pm 30_{-50}^{+70}$ & $11.7 \pm 2.7_{-4.7}^{+6.3}$ \\
\hline $\mathrm{J} 1810+1744$ & $4.2 \pm 0.5$ & $2.34 \pm 0.17$ & $1.9 \pm 0.2$ & $3.2 \pm 1.1$ & 442 & 23 & 3 & $112 \pm 8_{-30}^{+40}$ & $28.2 \pm 2.1_{-7.3}^{+9.4}$ \\
\hline $\mathrm{J} 1823-3021 \mathrm{~A}$ & $1.5 \pm 0.4$ & $1.07 \pm 0.15$ & $1.6 \pm 0.2$ & $2.5 \pm 0.6$ & 64 & 0 & 5 & $700 \pm 100 \pm 80$ & $8.9 \pm 1.3 \pm 0.9$ \\
\hline $\mathrm{J} 1858-2216$ & $0.55 \pm 0.28$ & $0.72 \pm 0.15$ & $0.84 \pm 0.74$ & $1.7 \pm 1.1$ & 75 & 17 & 0 & $8 \pm 2_{-2}^{+4}$ & $6.8 \pm 1.4_{-1.7}^{+3.2}$ \\
\hline J1902-5105 & $3.1 \pm 0.4$ & $2.16 \pm 0.15$ & $1.7 \pm 0.2$ & $3.4 \pm 1.1$ & 536 & 31 & 2 & $36 \pm 2 \pm 10$ & $5.2 \pm 0.4_{-1.1}^{+2.1}$ \\
\hline $\mathrm{J} 1939+2134 \dagger$ & $1.5 \pm 0.8$ & $0.9 \pm 0.3$ & - & - & 10 & 4 & 1 & $140 \pm 50 \pm 30$ & $1.3 \pm 0.5 \pm 0.2$ \\
\hline $\mathrm{J} 1959+2048$ & $2.4 \pm 0.5$ & $1.70 \pm 0.19$ & $1.4 \pm 0.3$ & $1.4 \pm 0.4$ & 185 & 43 & 0 & $130 \pm 10_{-40}^{+20}$ & $16.5 \pm 1.8_{-5.8}^{+2.2}$ \\
\hline $\mathrm{J} 2017+0603$ & $2.0 \pm 0.3$ & $3.33 \pm 0.21$ & $1.0 \pm 0.2$ & $3.4 \pm 0.6$ & 1196 & 100 & 1 & $98 \pm 6 \pm 20$ & $75.5 \pm 4.8 \pm 14$ \\
\hline $\mathrm{J} 2043+1711$ & $2.7 \pm 0.3$ & $2.70 \pm 0.16$ & $1.4 \pm 0.1$ & $3.3 \pm 0.7$ & 918 & 65 & 0 & $100 \pm 6_{-30}^{+20}$ & $79 \pm 5_{-26}^{+14}$ \\
\hline $\mathrm{J} 2047+1053$ & $0.83 \pm 0.36$ & $0.62 \pm 0.14$ & $1.5 \pm 0.5$ & $2.0 \pm 1.1$ & 69 & 11 & 2 & $31 \pm 7_{-8}^{+10}$ & $29.4 \pm 6.6_{-7.7}^{+9.9}$ \\
\hline $\mathrm{J} 2051-0827 \star$ & $0.24 \pm 0.13$ & $0.34 \pm 0.10$ & $0.50 \pm 0.76$ & $1.3 \pm 0.7$ & 68 & 15 & 0 & $4 \pm 1 \pm 1$ & $8.1 \pm 2.5 \pm 2.2$ \\
\hline $\mathrm{J} 2124-3358$ & $2.7 \pm 0.3$ & $3.68 \pm 0.16$ & $0.78 \pm 0.13$ & $1.63 \pm 0.19$ & 1993 & 237 & 3 & $4.0 \pm 0.2_{-1}^{+2}$ & $10.8 \pm 0.5_{-3.3}^{+5.6}$ \\
\hline $\mathrm{J} 2214+3000$ & $3.0 \pm 0.3$ & $3.28 \pm 0.15$ & $1.2 \pm 0.1$ & $2.2 \pm 0.3$ & 1689 & 146 & 2 & $93 \pm 4 \pm 20$ & $48.4 \pm 2.2 \pm 11$ \\
\hline $\mathrm{J} 2215+5135$ & $1.0 \pm 0.3$ & $1.18 \pm 0.14$ & $1.3 \pm 0.3$ & $3.4 \pm 1.0$ & 183 & 25 & 1 & $130 \pm 20 \pm 30$ & $24.6 \pm 3.0 \pm 5.7$ \\
\hline $\mathrm{J} 2241-5236$ & $3.0 \pm 0.3$ & $3.33 \pm 0.16$ & $1.3 \pm 0.1$ & $3.0 \pm 0.5$ & 2150 & 115 & 1 & $10.5 \pm 0.5 \pm 3$ & $4.0 \pm 0.2 \pm 1.1$ \\
\hline $\mathrm{J} 2302+4442$ & $2.6 \pm 0.3$ & $3.67 \pm 0.17$ & $0.94 \pm 0.12$ & $2.1 \pm 0.3$ & 1716 & 189 & 2 & $62 \pm 3_{-20}^{+10}$ & $162.6 \pm 7.5_{-57}^{+26}$ \\
\hline
\end{tabular}

Notes. Unbinned maximum likelihood spectral fit results for the LAT MSPs, using the PLEC1 model (Equation (12), Section 6). Columns 2 and 3 list the phase-averaged integral photon and energy fluxes in the 0.1 to $100 \mathrm{GeV}$ energy band, $F_{100}$ and $G_{100}$. Columns 4 and 5 list the photon index $\Gamma$ and cutoff energy $E_{\text {cut }}$. Columns 6, 7, and 8 list the source significance TS, significance $\mathrm{TS}_{\text {cut }}$ of the exponential cutoff compared to a simple power law, and significance $\mathrm{TS}_{\mathrm{b} \text { free }}$ of the PLEC compared to a PLEC1 shape. A value $\mathrm{TS}_{\text {cut }}<9$ indicates that a spectral cutoff is not significantly detected. Column 9 gives the total gamma-ray luminosity $L_{\gamma}$ in the 0.1 to $100 \mathrm{GeV}$ energy band. The gamma-ray conversion efficiency $\eta \equiv L_{\gamma} / \dot{E}^{\text {int }}$ in Column 10 assumes a beam correction factor $f_{\Omega}=1$ as described in Section 6.3, and the Shklovskii-corrected $\dot{E}^{\text {int }}$ values from Table 6 in Section 4.3. The first uncertainty in $L_{\gamma}$ and $\eta$ comes from the statistical uncertainties in the spectral fit, whereas the second is due to the distance uncertainty. The strong dependence of these quantities on distance (see Table 6) and beaming factor means that these values should be considered with care.

${ }^{a}$ A dagger ( $\dagger$ ) means the spectral fit is unreliable (see text). A star $(\star)$ means that the spectrum was calculated using the on-peak data only.

${ }^{\mathrm{b}}$ Overestimated distances or the assumed beaming factor, $f_{\Omega}=1$, can result in an efficiency $>100 \%$.

To estimate systematic uncertainties on the maximum likelihood spectral parameters we selected eight pulsars with representative characteristics (J0218+4232, J0248+6021, J0357+3205, J0614-3329, J0631+1036, J1658-5324, J18331034, and J1846+0919) and studied how their spectra changed when perturbing the Galactic diffuse emission and LAT effective area $\left(A_{\text {eff }}\right)$.

The distribution of Galactic diffuse normalization parameters, from all the fits, has a mean of 1.01 with $1 \sigma$ deviation of $4 \%$.
To estimate possible systematic effects due to an imperfect knowledge of this diffuse component, we repeated the spectral analysis with the normalization of the Galactic diffuse emission fixed to $(1 \pm 0.06)$ times the best-fit value, corresponding to $\pm 1.5 \sigma$ deviations. The average and largest deviations for $\Gamma$, $E_{\text {cut }}, F_{100}$, and $G_{100}$ from this test are listed in the first row of Table 11.

Systematic uncertainties on $A_{\text {eff }}$ are estimated to be $10 \%$ for $\log _{10} E / 1 \mathrm{MeV} \leqslant 2,5 \%$ for $\log _{10} E / 1 \mathrm{MeV}=2.75$, and $10 \%$ 
Table 11

Systematic Deviations on Pulsar Spectral Parameters

\begin{tabular}{|c|c|c|c|c|c|c|c|c|}
\hline Systematic & $\begin{array}{c}\langle\Delta \Gamma\rangle \\
(\%)\end{array}$ & $\begin{array}{c}\left\langle\Delta E_{\mathrm{cut}}\right\rangle \\
(\%)\end{array}$ & $\begin{array}{c}\left\langle\Delta F_{100}\right\rangle \\
(\%)\end{array}$ & $\begin{array}{c}\left\langle\Delta G_{100}\right\rangle \\
(\%)\end{array}$ & $\begin{array}{c}\max (\Delta \Gamma) \\
(\%)\end{array}$ & $\begin{array}{c}\max \left(\Delta E_{\text {cut }}\right) \\
(\%)\end{array}$ & $\begin{array}{c}\max \left(\Delta F_{100}\right) \\
(\%)\end{array}$ & $\begin{array}{c}\max \left(\Delta G_{100}\right) \\
(\%)\end{array}$ \\
\hline Galactic Diffuse & 14 & 4 & 16 & 12 & 80 & 27 & 65 & 46 \\
\hline Bracketing IRFs & 5 & 4 & 8 & 6 & 21 & 11 & 13 & 8 \\
\hline
\end{tabular}

for $\log _{10} E / 1 \mathrm{MeV} \geqslant 4$ with linear extrapolation in between, in log space (Ackermann et al. 2012a). To estimate the effects of these uncertainties we generated bracketing IRFs in which the usual $A_{\text {eff }}$ was replaced by,

$$
A_{\mathrm{B}}(E)=A_{\mathrm{eff}}(E)(1+\operatorname{err}(E) B(E)),
$$

where $\operatorname{err}(E)$ represents the $A_{\text {eff }}$ uncertainties with $B(E)=$ \pm 1 for the normalization factor $K$ and $B(E)=$ $\pm \tanh \left(\log _{10}\left(E / E_{0}\right) / \kappa\right)$ for $\Gamma$ and $E_{\text {cut }}$. Choosing $\kappa=0.13$ smoothes over twice the energy resolution. When using bracketing IRFs, it is important to isolate changes in the source of interest caused by the modified $A_{\text {eff }}$ from changes in the diffuse background spectrum introduced by this perturbation. Since the diffuse background spectra were derived from flight data, we multiply the spectrum of the Galactic diffuse model by $(1+\operatorname{err}(E) B(E))^{-1}$, ensuring that the predicted counts spectra from the diffuse background remains unchanged during the bracketing studies.

We integrate the fit results for the different bracketing IRFs to obtain $F_{100}$ and $G_{100}$. The second row of Table 11 lists the average and largest deviations for $\Gamma, E_{\text {cut }}, F_{100}$, and $G_{100}$ due to bracketing. $G_{100}$ is more robust than $F_{100}$. The considerably lower average deviations (columns 2 through 5) than maximum values (columns 6 through 9) shows that most deviations are much smaller than the outlying values.

Finally, to plot the spectra, we divided the 0.1 to $100 \mathrm{GeV}$ energy band into 4 (2) bins per decade for pulsars with TS above (below) 250 and fit a power law in each bin, curvature within the bins being negligible. We fixed the spectral parameters of all sources more than $4^{\circ}$ from the pulsar of interest and of the diffuse components at their full band fit results. The spectra of the pulsar of interest and the other point sources within the $4^{\circ}$ region were modeled as power laws with index fixed at 2 . Their flux levels in each energy band were obtained from a two-step fit. In the first step, sources with TS $\leqslant 0$ were removed, from that energy band only, as leaving them can adversely affect the fit uncertainties. The second step is to re-fit with the modified model. Sample spectra are shown in Figures 23(a)-(i) in Appendix A. For pulsars with $\mathrm{TS}_{\mathrm{b} \text { free }} \geqslant 9$, the figures show both the PLEC1 and PLEC fits. The spectra obtained for all pulsars with TS $\geqslant 25$ and reliable spectral fits are included in the online material.

\subsection{Spectral Results}

Table 9 lists the phase-averaged spectral results for the nonrecycled pulsars, and Table 10 for the MSPs. For ten pulsars, flagged with a $\uparrow$ symbol, the spectral fits were unreliable, for different reasons. Five are undetected as point sources, having TS $<25$. For two of these the likelihood analysis fails and we report no spectral parameters, as is the case for PSR J0729-1448 in spite of its larger TS value. For the other three the integrated flux is robust and we report $F_{100}$ and $G_{100}$. Similarly, we report only the integrated fluxes for four more pulsars, either because the maximum likelihood fit solution favored $\Gamma \approx 0$, or because the parameter uncertainties were of order $100 \%$.
For PSRs J1112-6103 and J1410-6132, analysis of the offpeak phase intervals (Section 7) showed significant extended emission, which we added to the phase-averaged source model, improving the spectral results.

Figure 7 shows correlation between $\Gamma$ and $\dot{E}$. The Pearson correlation factor is 0.68 for the young, radio-quiet pulsars and 0.58 for the MSPs, with probabilities of occurring by chance (two-sided $p$-values) of $5 \times 10^{-6}$ and $1.5 \times 10^{-4}$, respectively. For the young radio-loud pulsars, the correlation is smaller (0.40) and marginally significant (two-sided $p$-value of 0.017 ). For all pulsars together the correlation factor is 0.57 , with high significance $\left(2 \times 10^{-10}\right)$, even allowing for trials. We fit the measurements with $\Gamma=\mathcal{A} \log (\dot{E})+\mathcal{B}$ (dashed lines in the figure). For young pulsars we find similar trends in the radioloud and radio-quiet populations with $\mathcal{A} \approx 0.2$ and $\mathcal{B} \approx-5$. The exact fit values are sensitive to the outliers with small statistical uncertainties, such as the Crab. The MSPs have more dispersion in $\Gamma$ and a narrower $\dot{E}$ range, with a steeper slope than for the young population, $\mathcal{A} \approx 0.4$ and $\mathcal{B} \approx-12$.

In $1 \mathrm{PC}$ we noted a possible correlation between $E_{\text {cut }}$ and the magnetic field strength at the light cylinder $\left(B_{\mathrm{LC}}=\right.$ $4 \pi^{2}\left(1.5 I_{0} \dot{P}\right)^{1 / 2}\left(c^{3} P^{5}\right)^{-1 / 2}$, assuming an orthogonal rotator). For the radio-quiet pulsars, Figure 8 confirms the trend, with a Pearson correlation factor of 0.64 ( $p$-value $\left.4 \times 10^{-5}\right)$. The factor is 0.52 for the MSPs, with $p$-value 0.0007 . Here too, the correlation for the young, radio-loud pulsars is small (0.24) and insignificant ( $p$-value 0.17).

\subsection{Luminosity}

Gamma-ray emission models predict different relations between the spindown power $\dot{E}$ and the gamma-ray luminosity,

$$
L_{\gamma}=4 \pi d^{2} f_{\Omega} G_{100},
$$

making this a discriminating observable when applied to a large sample of gamma-ray pulsars. Following Romani \& Watters (2010), we define the beam correction factor $f_{\Omega}$ as

$$
f_{\Omega}\left(\alpha, \zeta_{E}\right)=\frac{\int F_{\gamma}(\alpha ; \zeta, \phi) \sin \zeta d \zeta d \phi}{2 \int F_{\gamma}\left(\alpha ; \zeta_{E}, \phi\right) d \phi}
$$

to extrapolate the observed flux to the full sky for some beam shape model. The angle $\alpha$ between the neutron star's magnetic and rotation axes is one model parameter. The angle $\zeta_{E}$ between the rotation axis and the Earth line of sight describes the inclination of the system relative to Earth. The numerator integrates emission into all space (all inclinations $\zeta$ ) whereas the denominator is the observed flux integrated over a neutron star rotation, with pulsar phase $\phi$. In the past, the gammaray beam was conventionally assumed to sweep out a $1 \mathrm{sr}$ solid angle, in which case $L_{\gamma}=d^{2} G_{100}$. Such a beam is appropriate to near-surface polar cap emission and corresponds to $f_{\Omega}=(1 / 4 \pi)=0.08$. An outer magnetosphere fan-like beam sweeping the entire sky ( $4 \pi$ steradians) gives $f_{\Omega} \approx 1$, which is the value we adopt for calculating $L_{\gamma}$. However, Pierbattista 


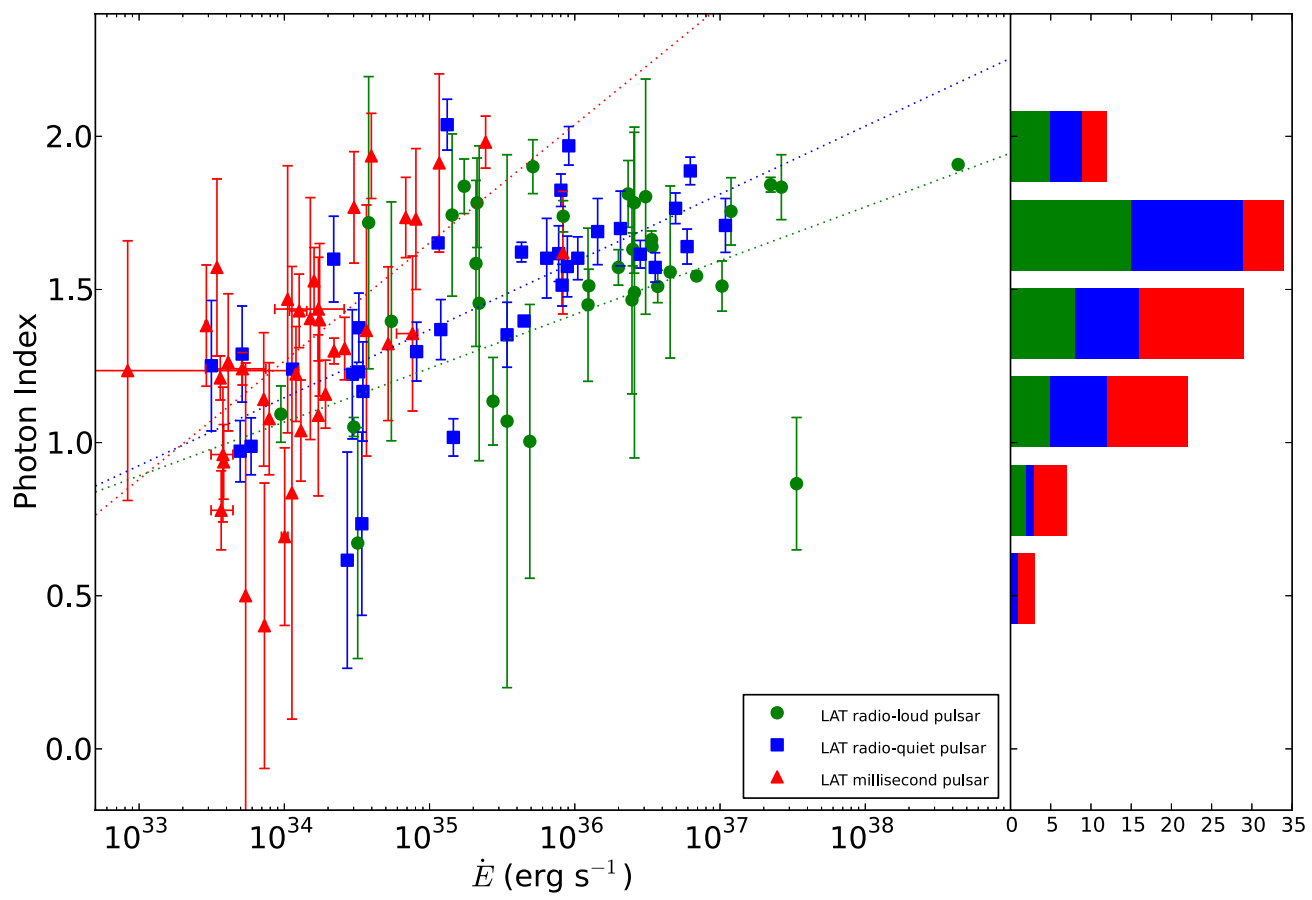

Figure 7. Power-law index $\Gamma$ for the exponentially cutoff gamma-ray spectra vs. the Shklovskii-corrected spindown power, for the pulsars bright enough in gamma rays to allow spectral analysis (see text). The straight-line fit results are in Section 6.2. The markers are the same as in Figure 1. The uppermost line is for millisecond gamma-ray pulsars. The middle line fits young, radio-quiet gamma-ray pulsars, while the lowest line is for young, radio-loud gamma-ray pulsars. A histogram of photon index values is projected onto the axis.

(A color version of this figure is available in the online journal.)

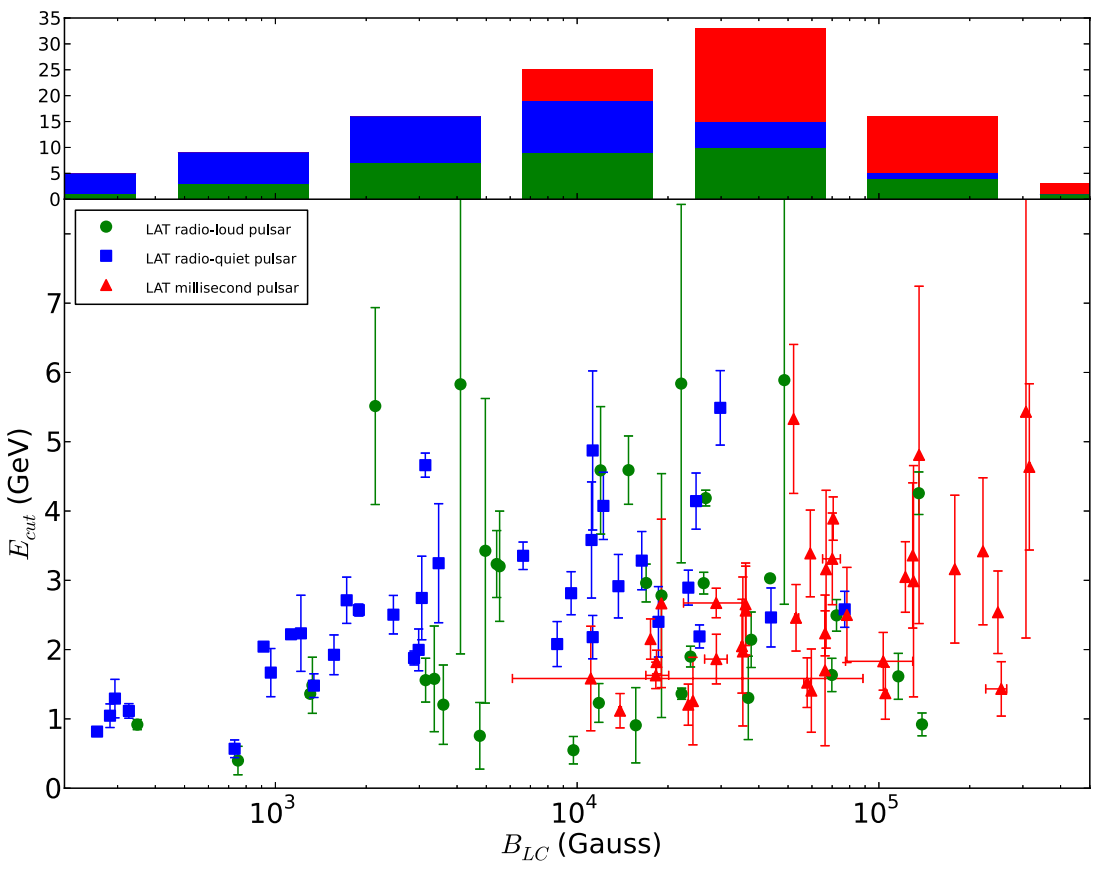

Figure 8. Best-fit cutoff energy vs. magnetic field at the light cylinder, $B_{\mathrm{LC}}$. The young, radio-quiet gamma-ray pulsars have lower $B_{\mathrm{LC}}$ than other gamma-ray pulsars. The markers are the same as in Figure 1. The histogram highlights the different $B_{\mathrm{LC}}$ distributions for the three pulsar classes.

(A color version of this figure is available in the online journal.)

et al. (2012) found a large spread in $f_{\Omega}$ values for different emission models and for radio-loud versus radio-quiet young pulsars. Values of $f_{\Omega}$ exceeding 1 correspond to beams that are narrow in $\phi$, extended in $\zeta$, and/or have average intensity exceeding the value sampled at $\zeta_{E}$.

Figure 9 shows $L_{\gamma}$ versus $\dot{E}$. Pulsars with poor spectral fits have been excluded. The open field-line voltage is $V \simeq$
$3.18 \times 10^{-3} \sqrt{\dot{E}}$ volts. Above some threshold voltage, gammaray emitting electron-positron cascades occur, and a linear dependence of $L_{\gamma}$ on $V$ would give $L_{\gamma} \propto \sqrt{\dot{E}}$ (Arons 1996), as for the lower diagonal line. With arbitrary normalization, we call this the "heuristic" gamma-ray pulsar luminosity,

$$
L_{\gamma}^{h}=10^{33} \sqrt{\dot{E} / 10^{33}}=\sqrt{10^{33} \dot{E}} \operatorname{erg~s}^{-1} .
$$




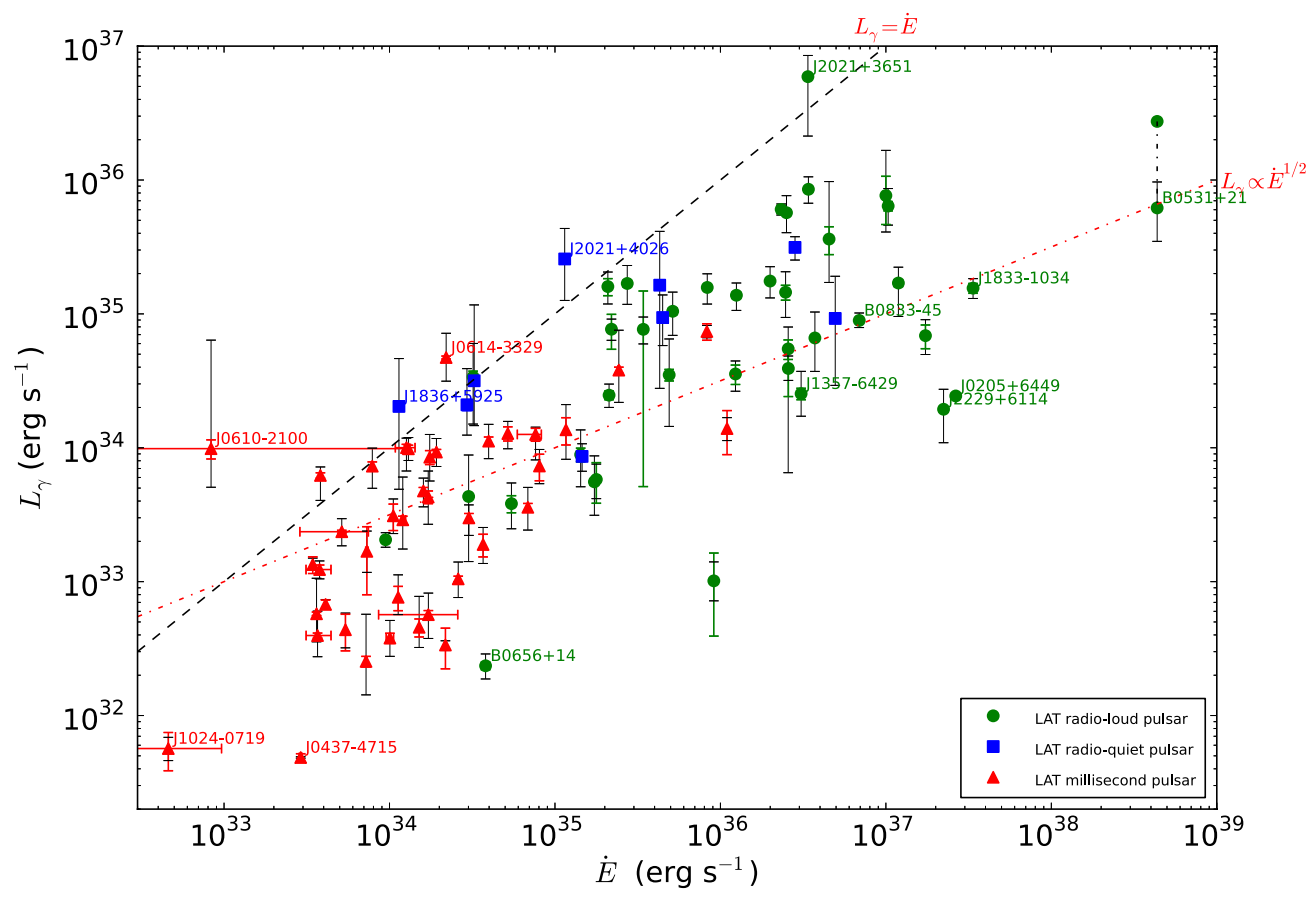

Figure 9. Gamma-ray luminosity $L_{\gamma}=4 \pi f_{\Omega} d^{2} G_{100}$ in the 0.1 to $100 \mathrm{GeV}$ energy band vs. spindown power $\dot{E}$. The vertical error bars from the statistical uncertainty on the energy flux $G_{100}$ are colored in the online journal. The vertical error bars due to the distance uncertainties are black, and generally larger. Doppler corrections (Section 4.3) have been applied to MSPs with known proper motions, leading to visible horizontal error bars in some cases. The upper diagonal line indicates $100 \%$ conversion of spindown power into gamma-ray flux: for pulsars above this line, the distance $d$ may be smaller, and/or the assumed beam correction $f_{\Omega} \equiv 1$ is wrong. The lower diagonal line indicates the heuristic luminosity $L_{\gamma}^{h}=\sqrt{10^{33} \dot{E}} \mathrm{erg} \mathrm{s}^{-1}$, to guide the eye. The upper of the two Crab points, at far right, includes the X-ray energy flux (see Section 9.1). The markers are the same as in Figure 1.

(A color version of this figure is available in the online journal.)

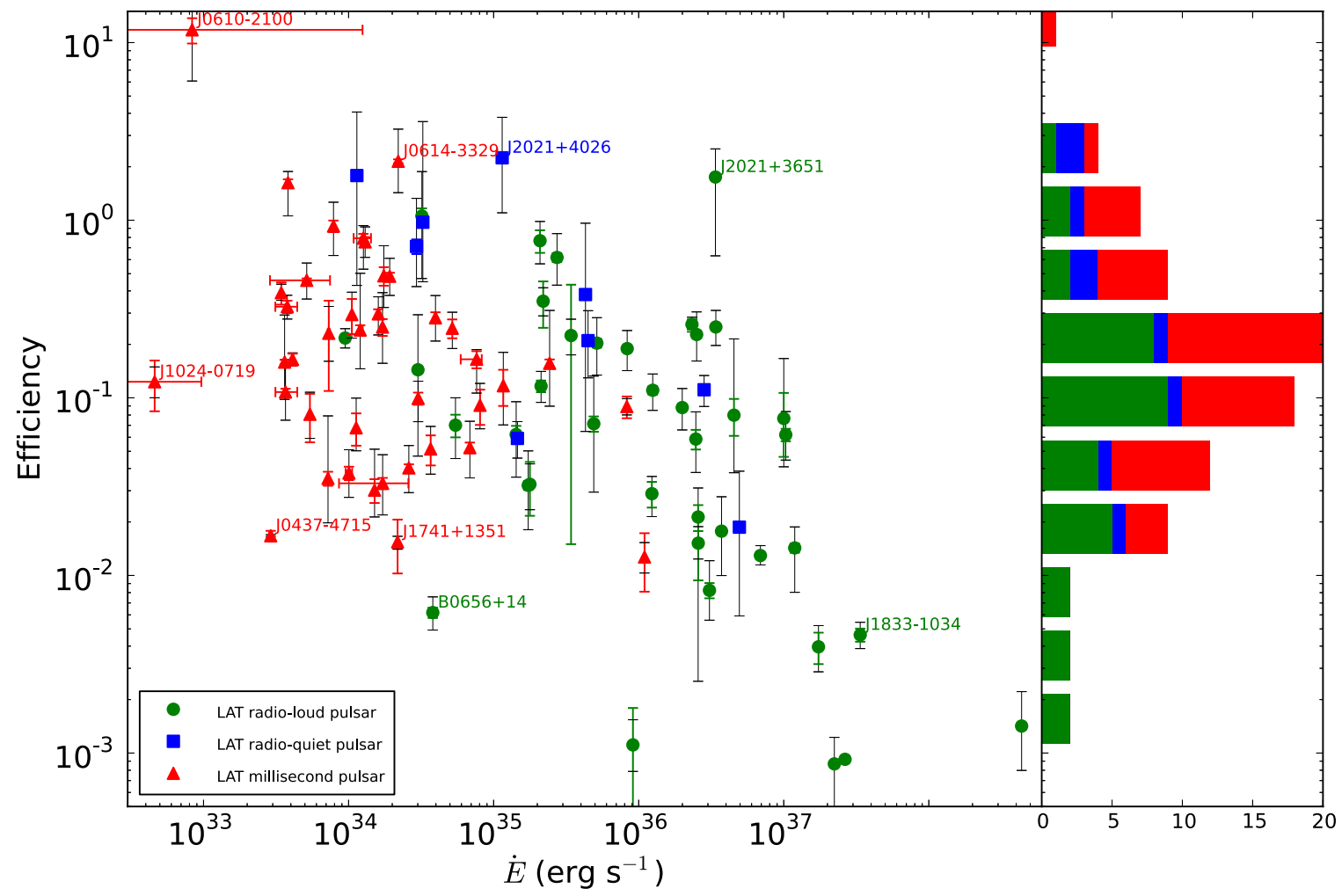

Figure 10. Gamma-ray efficiency $\eta=L_{\gamma} / \dot{E}$ vs. spindown power $\dot{E}$. The error bars are as in Figure 9. The markers and the side histogram use the same color coding as in Figure 1.

(A color version of this figure is available in the online journal.) 

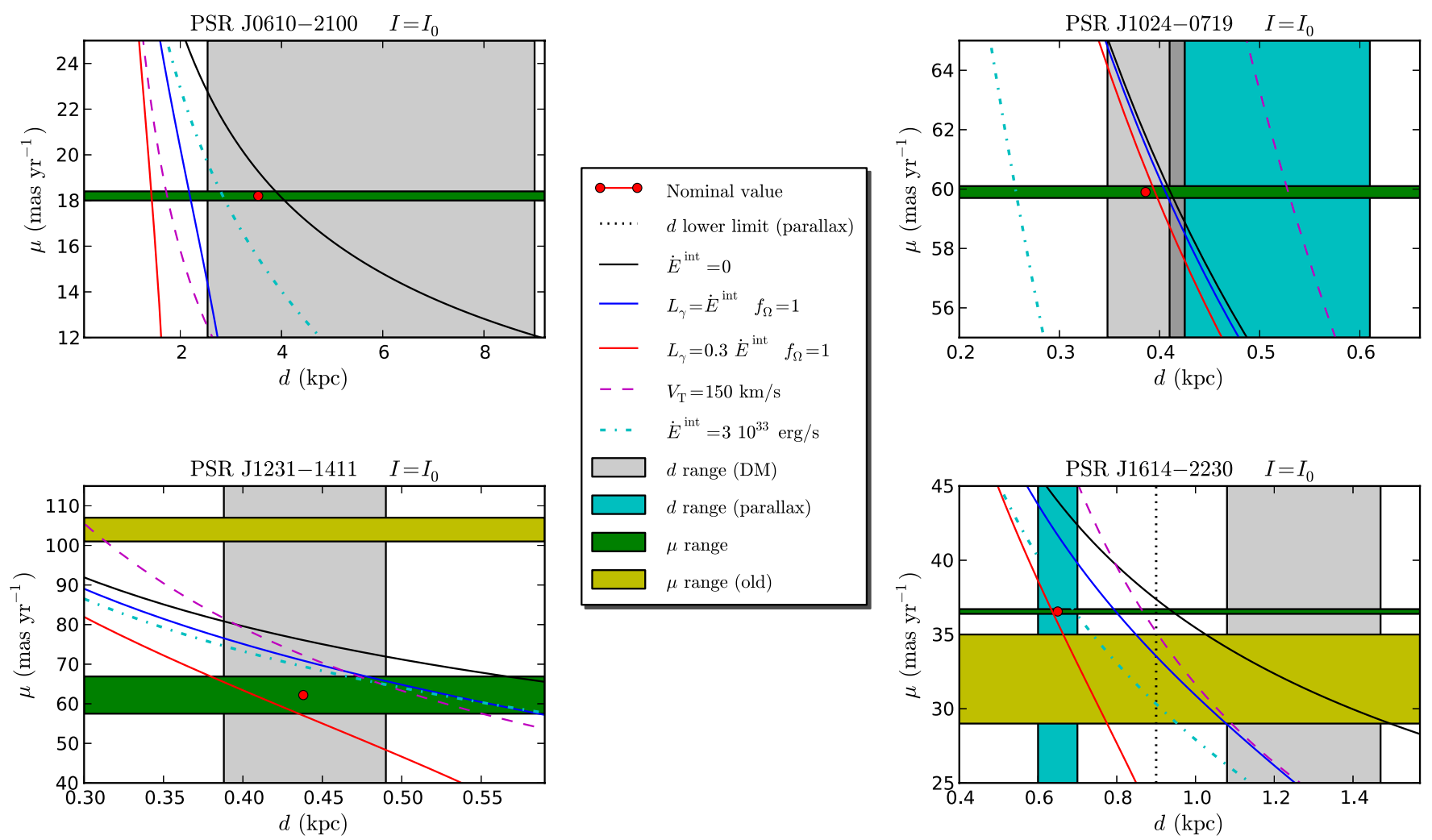

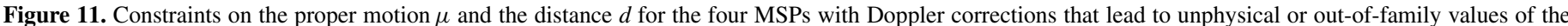

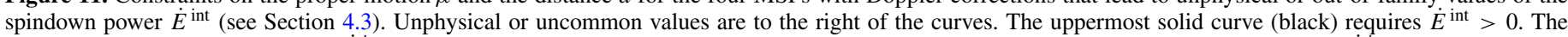

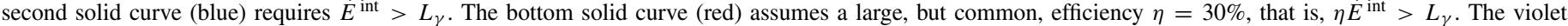

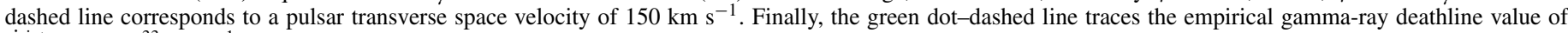

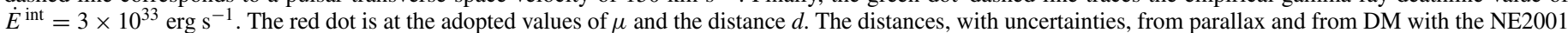

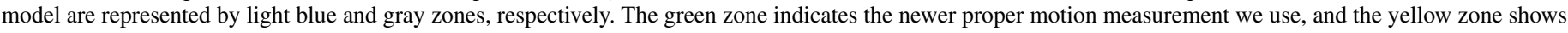
earlier values. The black dotted line shows the lower limit of parallax distance for J1614-2230 from Demorest et al. (2010).

(A color version of this figure is available in the online journal.)

At low $\dot{E}$ values $L_{\gamma}$ seems to be falling below $L_{\gamma}^{h}$. The upper diagonal line shows $L_{\gamma}=\dot{E}$, that is, a $100 \%$ efficiency $\eta=L_{\gamma} / \dot{E}$ for converting spindown power into gamma rays. A few pulsars appear above this line, likely due to over-estimated distances or $f_{\Omega}$ values. Figure 10 plots $\eta$ versus $\dot{E}$. Overall, the expected $\eta \propto 1 / \sqrt{\dot{E}}$ trend is roughly respected. However, the large dispersion due to the distance uncertainties, as well as beaming effects, limits the extent to which the data constrain the theory.

The Doppler correction to $\dot{E}$ is small $(|\xi|<10 \%)$ for 9 of the 20 pulsars with proper motion corrections (Table 6). For the five with $20 \%<\xi<60 \%$, the correction refines their positions in e.g., the $L_{\gamma}$ versus $\dot{E}^{\text {int }}$ plane. The Doppler correction for PSR J0437-4715, with a large $\xi=75 \%$, produces a qualitative change: observed $\dot{E}=12 \times 10^{33} \mathrm{erg} \mathrm{s}^{-1}$ decreases to corrected $\dot{E}^{\mathrm{int}}=3 \times 10^{33} \mathrm{erg} \mathrm{s}^{-1}$, right at the apparent deathline, and the efficiency changes from the lowest outlier amongst MSPs, to a low, but typical, $\eta=1.7 \%$.

The remaining four pulsars with $\xi>60 \%$ bear special discussion. Figure 11 plots lines of constant $\dot{E}, L_{\gamma}$, and transverse velocity $v_{T}$ in $\mu$ versus $d$ space for different assumptions: $\dot{E}=0$, $\dot{E}=L_{\gamma}$, and $\eta \dot{E}=L_{\gamma}$ with $\eta=30 \%$, at the high end of the observed range. The curve for $v_{T}=\mu d=150 \mathrm{~km} \mathrm{~s}^{-1}$ is the $3 \sigma$ extremum of the MSP velocity distribution of Lyne et al. (1998). Faster recycled pulsars are possible, but unusual. Allowed (or favored) regions are to the left of the curves. The curve for $\dot{E}=3 \times 10^{33} \mathrm{erg} \mathrm{s}^{-1}$ shows how an $\dot{E}$ value lower than those seen to date would compare with the other constraints. The shaded zones correspond to the measurements and their uncertainties adopted in this paper along with previous measurements, for comparison. We recall that here, as throughout the paper, we adopt the moment of inertia $I_{0}=10^{45} \mathrm{gm} \mathrm{cm}^{2}$, corresponding to a neutron star mass of $1.4 M_{\odot}$ and radius of $10 \mathrm{~km}$.

PSR J0610-2100 was recently discussed by Espinoza et al. (2013): the intrinsic spindown power is well below the empirical deathline, the space velocity is much higher than typical, and the gamma-ray efficiency exceeds 100\%. In Figure 11 the nominal $(\mu, d)$ point for this pulsar is to the right of most of the curves. These apparent paradoxes are resolved if the pulsar is closer than $2 \mathrm{kpc}$. Upon inspection of NE2001, minor changes in the "Local Super Bubble" shape and density for this line of sight could yield a distance less than $2 \mathrm{kpc}$. Espinoza et al. (2013) also describe material along the line of sight that is unmodeled in NE2001.

They also discussed PSR J1024-0719. We show both the corrected parallax distance from Verbiest et al. (2012) as well as the NE2001 distance, for which the $10 \%$ uncertainty is probably underestimated given the small $\mathrm{DM}=6.5 \mathrm{pc} \mathrm{cm}^{-3}$ in that direction. However, any distance compatible with the parallax yields the lowest intrinsic spindown power $\dot{E}^{\text {int }}$ of any gammaray pulsar, and well below the $3 \times 10^{33} \mathrm{erg} \mathrm{s}^{-1}$ current minimum.

For PSR J1231-1411, discovered in an unassociated LAT source, Ransom et al. (2011) measured a large proper motion, $\mu>100$ mas $\mathrm{yr}^{-1}$, indicated in Figure 11. The Doppler correction using that large $\mu$ gave negative $\dot{E}^{\text {int }}$ values, which 
is unphysical. Radio observations since the initial measurement allowed us to update the timing model, resulting in the smaller proper motion value listed in Table 6 and plotted in Figure 11. The Doppler corrections now lead to rather typical parameters for this pulsar.

PSR J1614-2230 is one of the rare pulsars with a neutron star mass measurement. Demorest et al. (2010) measured the Shapiro delay precisely in this binary system to obtain $M=1.97 \pm 0.04 M_{\odot}$, well above the Chandrasekhar mass of $1.4 M_{\odot}$. The DM distance, $1.27 \mathrm{kpc}$, along with the proper motion measured in the same paper yields implausible $\dot{E}^{\text {int }}$ and $\eta$ values. A moment of inertia greater than $I_{0}$ would improve the situation, favoring "rigid" neutron star equations-of-state. However, the parallax distance and proper motion recently measured by Lassus (2013) brings this pulsar back in line with the rest of the population. ${ }^{100}$

Demorest et al. (2010) conclude that $M>1.4 M_{\odot}$ is probably true for many or most MSPs. For $I>I_{0}, \dot{E}^{\text {int }}$ is larger than the "standard" values in Table 6 . This would shrink the spread between the $\dot{E}^{\text {int }}$ distributions for the young and MSP gammaray pulsars.

\section{UNPULSED MAGNETOSPHERIC EMISSION}

Some pulsars have magnetospheric emission over their full rotation phase with similar spectral characteristics to the emission seen through their peaks. This emission appears in the observed light curves as a low-level, unpulsed component above the estimated background level (i.e., not attributable to diffuse emission or nearby point sources) and can be a powerful discriminator for the emission models.

On the other hand a PWN around the pulsar, or a photon excess due to imprecise knowledge of diffuse emission around the pulsar, would not be modulated at the rotational period and could be confused with a constant magnetospheric signal. Including the discovery of the GeV PWN 3C 58 associated with PSR J0205+6449 described in this section, the LAT sees 17 sources potentially associated with $\mathrm{PWNe}$ at $\mathrm{GeV}$ energies (Acero et al. 2013a). Some are highlighted in Appendix C. This off-peak emission should be properly modeled when searching for pulsar emission at all rotation phases.

We can discriminate between these two possible signals through spectral and spatial analysis. If the emission is magnetospheric, it is more likely to appear as a non-variable point source with an exponentially cutoff spectrum with a well-known range of cutoff energies. On the other hand, PWNe and diffuse excesses have spectra with a power-law shape and either a hard index continuing up to tens of $\mathrm{GeV}$ in the PWN case or present only at lower energies with a very soft index in the diffuse case. In addition, PWNe are often spatially resolvable at $\mathrm{GeV}$ energies (e.g., Vela-X has been spatially resolved with the LAT and AGILE and HESS J1825-137 with the LAT; Abdo et al. 2010e; Pellizzoni et al. 2010; Grondin et al. 2011, respectively) so an extended source would argue against a magnetospheric origin of the emission. However, given the finite angular resolution of the LAT (see Section 2) not all PWNe will appear spatially extended at GeV energies. The Crab Nebula, for instance, cannot be resolved by the LAT but can be distinguished from the gamma-bright Crab pulsar, in the off-peak interval, by its hard spectrum above $\sim 1 \mathrm{GeV}$ (Abdo et al. 2010c). In addition, GeV emission from the Crab Nebula was discovered

\footnotetext{
${ }^{100}$ The proper motion to be reported in Lassus (2013) is at present smaller than
} that in Table 6, and consistent with the value in Demorest et al. (2010). to be time-variable (e.g., Abdo et al. 2011a) providing another possible way to discern the nature of any observed off-peak signal.

Therefore, to identify pulsars with magnetospheric emission across the entire rotation, we define and search the off-peak intervals of the pulsars in this catalog for significant emission, except PSR J2215+5135 for which the rotation ephemeris covers a short time interval and the profile is noisy. We then evaluate the spectral and spatial characteristics of any off-peak emission to determine if it is likely magnetospheric, related to the pulsar wind, or physically unrelated to the pulsar (e.g., unmodeled diffuse emission).

\subsection{Off-peak Phase Selection}

We first developed a systematic, model-independent, and computationally-efficient method to define the off-peak interval of a pulsar light curve.

We begin by deconstructing the light curve into simple Bayesian Blocks using the algorithm described in Jackson et al. (2005) and Scargle et al. (2013). We could not apply the Bayesian Block algorithm to the weighted-counts light curves because they do not follow Poisson statistics, required by the algorithm. We therefore use an unweighted-counts light curve in which the angular radius and minimum energy selection have been varied to maximize the $H$-test statistic. To produce Bayesian Blocks on a periodic light curve, we extend the data over three rotations, by copying and shifting the observed phases to cover the phase range from -1 to 2 . We do, however, define the final blocks to be between phases 0 and 1 . To avoid potential contamination from the trailing or leading edges of the peaks, we reduce the extent of the block by $10 \%$ on either side, referenced to the center of the block.

There is one free parameter in the Bayesian Block algorithm

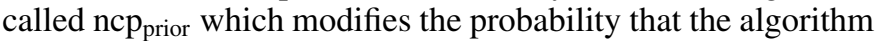
will divide a block into smaller intervals. We found that, in most cases, setting ncp $p_{\text {prior }}=8$ protects against the Bayesian Block decomposition containing unphysically small blocks. For a few marginally-detected pulsars, the algorithm failed to find more than one block and we had to decrease ncp prior until the algorithm found a variable light curve. Finally, for a few pulsars the Bayesian-block decomposition of the light curves failed to model weak peaks found by the light-curve fitting method presented in Section 5.2 or extended too far into the other peaks. For these pulsars, we conservatively shrink the off-peak region.

For some pulsars, the observed light curve has two wellseparated peaks with no significant bridge emission, which leads to two well-defined off-peak intervals. We account for this possibility by finding the second-lowest Bayesian block and accepting it as a second off-peak interval if the emission is consistent with that in the lowest block (at the $99 \%$ confidence level) and if the extent of the second block is at least half that of the first block.

Figure 12 shows the energy-and-radius optimized light curves, the Bayesian block decompositions, and the off-peak intervals for six pulsars. Figures 22(a)-(h) overlay off-peak intervals over the weighted light curves of several pulsars. The off-peak intervals for all pulsars in this catalog are given in Tables 7 and 8.

\subsection{Off-peak Analysis Method}

Characterizing both the spatial and spectral characteristics of any off-peak emission helps discern its origin. We employ a 

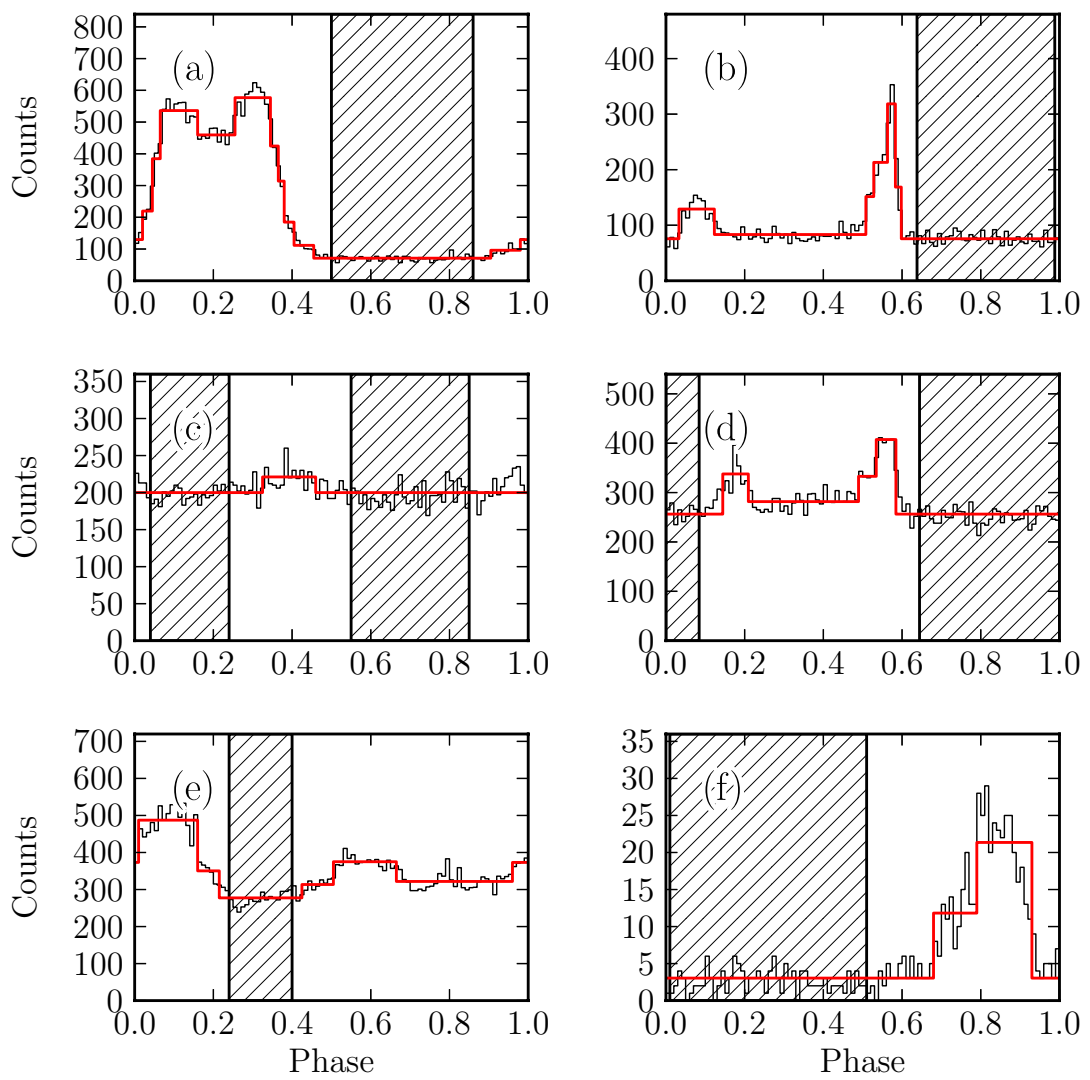

Figure 12. Energy-and-radius optimized light curve, Bayesian block decomposition of the light curve, and off-peak interval for (a) PSR J0007+7303, (b) PSR J0205+6449, (c) PSR J1410-6132, (d) PSR J1747-2958, (e) PSR J2021+4026, and (f) PSR J2124-3358. The black histograms represent the light curves, the gray lines (colored red in the electronic version) represent the Bayesian block decompositions of the pulsar light curves, and the hatched areas represent the off-peak intervals selected by this method.

(A color version of this figure is available in the online journal.)

somewhat different analysis procedure here than for the phaseaveraged analysis described in Section 6.1. To evaluate the spatial characteristics of any off-peak emission we use the likelihood fitting package pointlike (detailed in Lande et al. 2012), and to fit the spectrum we use gtlike in binned mode via pyLikelihood as was done for the phase-averaged analysis.

For each pulsar we start from the same temporal and spatial event selections described in Section 2 but we increase the maximum energy to $400 \mathrm{GeV}$ (the highest event energy for any ROI under this selection is $\sim 316 \mathrm{GeV}$ ). For the pointlike analysis we further select a $10^{\circ}$ radius ROI and for gtlike a $14^{\circ} \times 14^{\circ}$ square ROI, both centered on the pulsar position. Finally, we only consider photons with pulse phases within the corresponding off-peak interval.

We search for off-peak emission assuming a point source and (except for the Crab Nebula and Vela-X, described below) a power-law spectral model. We fit the position of this putative off-peak source using pointlike as described by Nolan et al. (2012) and then use the best-fit position in a spectral analysis with gtlike. From the spectral analysis we require $\mathrm{TS} \geqslant 25$ (just over $4 \sigma$ ) to claim a detection. If $\mathrm{TS}<25$, we compute upper limits on the flux in the energy range from $100 \mathrm{MeV}$ to $316 \mathrm{GeV}$ assuming a power law with photon index fixed to 2.0 and a PLEC1 model with $\Gamma=1.7$ and $E_{\text {cut }}=3 \mathrm{GeV}$.

The spectrum of the Crab Nebula (associated with PSR J0534+2200) is uniquely challenging because the $\mathrm{GeV}$ spectrum contains both a falling synchrotron and a rising inverse Compton component (Abdo et al. 2010c). For this particular source we used the best-fit two-component spectral model from
Buehler et al. (2012) and fit only the overall normalization of the source. In addition, for Vela-X (associated with PSR J08354510) we took the best-fit spectral model from Grondin et al. (2013) and fit only the overall normalization of this source. This spectrum has a smoothly broken power law spectral model and was fit assuming Vela-X to have an elliptical disk spatial model.

If the off-peak source is significant, we test whether the spectrum shows evidence for a cutoff, as described in Section 6.1 and by Ackermann et al. (2011), assuming the source is at the pulsar position. We say that the off-peak emission shows evidence for a cutoff if $\mathrm{TS}_{\text {cut }} \geqslant 9$, corresponding to a $3 \sigma$ detection.

For a significant off-peak point source, we use pointlike to test if the emission is significantly extended. We assume a radially-symmetric Gaussian source and fit the position and extension parameter $(\sigma)$ as described in Lande et al. (2012). The best-fit extended source parameters are then given to gtlike, which is used to fit the spectral parameters and the significance of the extension over a point source, $\mathrm{TS}_{\mathrm{ext}}$, evaluated as described in Lande et al. (2012). That paper established that $\mathrm{TS}_{\mathrm{ext}} \geqslant 16$ means highly probable source extension. In the present work we aim only to flag possible extension, and use $\mathrm{TS}_{\text {ext }} \geqslant 9$.

To test for variability, even without significant emission over the three-year time range, we divide the dataset into 36 intervals and fit the point-source flux independently in each interval, computing $\mathrm{TS}_{\mathrm{var}}$ as in 2FGL. For sources with potential magnetospheric off-peak emission and for regions with no detection, we performed the test at the pulsar's position. 


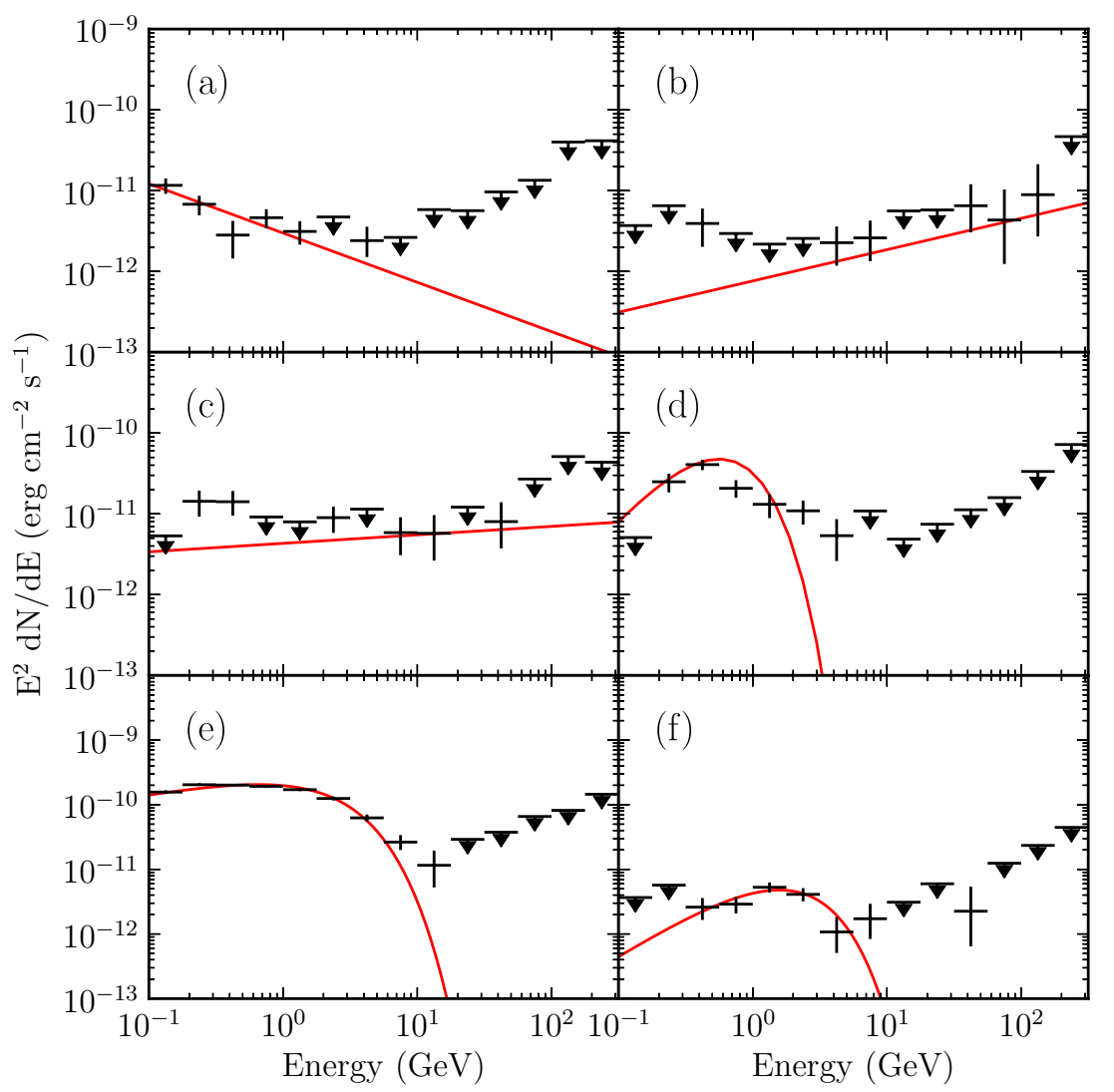

Figure 13. Spectral energy distributions for the off-peak phase intervals around (a) PSR J0007+7303, (b) PSR J0205+6449, (c) PSR J1410-6132, (d) PSR J1747-2958, (e) PSR J2021+4026, and (f) PSR J2124-3358. We plot a detection in those energy bands in which the source is found with TS $\geqslant 4$ (a $2 \sigma$ detection) and report a Bayesian 95\% confidence-level upper limit otherwise. The best-fit spectral model, using the full energy range, is also shown for comparison.

(A color version of this figure is available in the online journal.)

Otherwise, we test at the best-fit position. The off-peak emission is said to show evidence for variability if $\mathrm{TS}_{\mathrm{var}} \geqslant 91.7$, corresponding to a $4 \sigma$ significance. As noted in Section 2, our timing solutions for PSRs J0205+6449 and J1838-0537 are not coherent across all three years. For these two pulsars, we excluded the time ranges without ephemerides and only tested for variability during months that were completely covered. For $\mathrm{J} 1838-0537$ only one month is lost, whereas for J0205+6449 the $7 \%$ data loss is spread across three separate months. As a result, $\mathrm{TS}_{\mathrm{var}}$ for these pulsars is a conservative estimate of variability significance.

The procedure described above, especially the extension analysis, is particularly sensitive to sources not included in 2FGL that are near the pulsar of interest, for two reasons. First, we are using an additional year of data and second, when "turning off" a bright pulsar nearby, faint sources become more important to the global fit. Therefore, in many situations we had to run the analysis several times, iteratively improving the model by including new sources, until we removed all TS $>25$ residuals. The final gtlike-formatted XML source model for each off-peak region is included in the auxiliary material.

There are still, however, pulsars for which we were unable to obtain an unbiased fit of the off-peak emission, most likely due to inaccuracies in the model of the Galactic diffuse emission and incorrectly modeled nearby sources. The most common symptom of a biased fit is an unphysically large extension. In these cases, the extended source attempts to account for multiple point sources or incorrectly-modeled diffuse emission, not just the putative off-peak emission. Systematics associated with modeling extended sources are discussed more thoroughly in Lande et al. (2012). For the purposes of this catalog, we have flagged the pulsars where off-peak analysis suffered from these issues and do not attempt a complete understanding of the emission.

\subsection{Off-peak Results}

The off-peak intervals of 54 LAT-detected pulsars have been evaluated by Ackermann et al. (2011) using 16 months of sky survey observations. This led to the discovery of PWNlike emission in the off-peak interval of PSR J1023-5746, coincident with HESS J1023-575, and identification of 5 pulsars that appear to have near $100 \%$ duty cycles. Our results, summarized in Table 12, extend the analysis to 116 pulsars over three years of data. Sample off-peak spectra are shown in Figure 13. Using the procedures outlined in Sections 7.1 and 7.2, we have identified 34 pulsars that have significant emission $(\mathrm{TS} \geqslant 25)$ in their off-peak intervals. We classify the likely nature of the emission as follows.

If the spectrum cuts off $\left(\mathrm{TS}_{\mathrm{cut}} \geqslant 9\right)$, the emission could be magnetospheric ("M"). An indication of spatial extension $\left(\mathrm{TS}_{\mathrm{ext}} \geqslant 9\right)$ flags sources where the emission may instead be an artifact of defects in the Galactic diffuse emission model and we list such sources as type "U," for "unidentified." Similarly, emission from sources without evidence for a spectral cutoff could originate in the pulsar wind, type "W." Spatial extension alone is not a sufficient indicator, since the LAT PSF is larger than many PWNe. A hard spectral index also suggests a PWN contribution. The table lists the four solid PWN detections. PSR J0205+6449 in 3C58 is a new detection at GeV energies. 
Table 12

Off-peak Spatial and Spectral Results

\begin{tabular}{|c|c|c|c|c|c|c|c|}
\hline PSR & Type & TS & $\mathrm{TS}_{\mathrm{ext}}$ & $\mathrm{TS}_{\text {cutoff }}$ & $\begin{array}{c}\text { Energy Flux } \\
\left(10^{-11} \mathrm{erg} \mathrm{cm}^{-2} \mathrm{~s}^{-1}\right)\end{array}$ & $\Gamma$ & $\begin{array}{l}E_{\text {cutoff }} \\
(\mathrm{GeV})\end{array}$ \\
\hline \multicolumn{8}{|c|}{ Young Pulsars } \\
\hline $\mathrm{J} 0007+7303$ & $\mathrm{U}$ & 71.4 & 10.8 & 0.0 & $1.98 \pm 0.26$ & $2.61 \pm 0.14$ & \\
\hline J0205+6449 & W & 33.7 & 0.5 & 0.0 & $1.75 \pm 0.68$ & $1.61 \pm 0.21$ & \\
\hline $\mathrm{J} 0534+2200$ & W & 5247. & 0.0 & 0.3 & $67.2 \pm 1.6$ & a & \\
\hline $\mathrm{J} 0631+1036$ & $\mathrm{U}$ & 33.1 & 0.0 & 5.4 & $1.70 \pm 0.33$ & $2.38 \pm 0.14$ & \\
\hline J0633+1746 & M & 3666. & 2.3 & 239. & $41.4 \pm 1.1$ & $1.37 \pm 0.09$ & $0.93 \pm 0.10$ \\
\hline J0734-1559 & $\mathrm{U}$ & 28.3 & 12.4 & 30.8 & $1.61 \pm 0.24$ & $0.01 \pm 0.08$ & $0.17 \pm 0.03$ \\
\hline J0835-4510 & W & 473. & 283. & 22.8 & $30.3 \pm 1.2$ & b & \\
\hline J0908-4913 & $\mathrm{U}$ & 65.1 & 41.4 & 60.4 & $3.04 \pm 1.07$ & $0.15 \pm 0.59$ & $0.30 \pm 0.01$ \\
\hline $\mathrm{J} 1023-5746$ & $\mathrm{U}$ & 59.7 & 30.0 & 10.9 & $5.35 \pm 1.17$ & $0.57 \pm 0.80$ & $0.49 \pm 0.21$ \\
\hline J1044-5737 & $\mathrm{U}$ & 42.0 & 98.1 & 22.4 & $3.12 \pm 0.75$ & $0.80 \pm 0.93$ & $0.40 \pm 0.18$ \\
\hline J1105-6107 & $\mathrm{U}$ & 33.3 & 37.5 & 21.7 & $3.81 \pm 0.77$ & $0.92 \pm 0.56$ & $0.48 \pm 0.22$ \\
\hline J1112-6103 & $\mathrm{U}$ & 65.0 & 71.1 & 0.9 & $5.10 \pm 0.74$ & $2.17 \pm 0.09$ & \\
\hline J1119-6127 & $\mathrm{U}$ & 61.3 & 1.0 & 0.9 & $4.11 \pm 0.63$ & $2.22 \pm 0.09$ & \\
\hline J1124-5916 & M & 95.9 & 0.0 & 18.2 & $2.87 \pm 0.71$ & $1.31 \pm 0.91$ & $1.43 \pm 1.42$ \\
\hline $\mathrm{J} 1410-6132$ & $\mathrm{U}$ & 27.5 & 71.2 & 0.4 & $4.29 \pm 1.05$ & $1.90 \pm 0.15$ & \\
\hline J1513-5908 & W & 102. & 3.5 & 0.0 & $4.95 \pm 0.83$ & $1.78 \pm 0.12$ & \\
\hline J1620-4927 & M & 28.9 & 0.5 & 35.2 & $5.25 \pm 0.96$ & $0.35 \pm 0.94$ & $0.57 \pm 0.29$ \\
\hline J1746-3239 & $\mathrm{U}$ & 53.3 & 34.3 & 34.2 & $3.65 \pm 0.59$ & $0.94 \pm 0.31$ & $0.60 \pm 0.10$ \\
\hline $\mathrm{J} 1747-2958$ & M & 45.5 & 5.4 & 49.8 & $8.41 \pm 2.84$ & $0.02 \pm 0.32$ & $0.28 \pm 0.01$ \\
\hline $\mathrm{J} 1809-2332$ & $\mathrm{U}$ & 32.5 & 13.6 & 21.9 & $4.10 \pm 0.80$ & $0.24 \pm 0.83$ & $0.31 \pm 0.11$ \\
\hline $\mathrm{J} 1813-1246$ & M & 62.8 & 0.0 & 9.0 & $6.31 \pm 1.40$ & $1.60 \pm 0.73$ & $0.99 \pm 0.95$ \\
\hline $\mathrm{J} 1836+5925$ & M & 10407. & 0.0 & 365. & $36.9 \pm 0.7$ & $1.47 \pm 0.03$ & $1.98 \pm 0.09$ \\
\hline $\mathrm{J} 1838-0537$ & $\mathrm{U}$ & 51.3 & 32.9 & 21.9 & $8.35 \pm 1.31$ & $1.39 \pm 0.54$ & $2.55 \pm 2.48$ \\
\hline $\mathrm{J} 2021+4026$ & M & 1717. & 8.7 & 244. & $64.0 \pm 1.4$ & $1.64 \pm 0.02$ & $1.82 \pm 0.04$ \\
\hline $\mathrm{J} 2032+4127$ & $\mathrm{U}$ & 53.6 & 76.1 & 1.5 & $4.36 \pm 0.77$ & $2.07 \pm 0.12$ & \\
\hline $\mathrm{J} 2055+2539$ & M & 123. & 0.0 & 30.0 & $1.63 \pm 0.19$ & $1.05 \pm 0.28$ & $0.64 \pm 0.12$ \\
\hline \multicolumn{8}{|c|}{ Millisecond Pulsars } \\
\hline J0034-0534 & $\mathrm{U}$ & 41.0 & 0.0 & 6.0 & $0.82 \pm 0.16$ & $2.40 \pm 0.19$ & \\
\hline J0102+4839 & $\mathrm{U}$ & 49.7 & 0.0 & 7.4 & $1.29 \pm 0.20$ & $2.51 \pm 0.14$ & \\
\hline $\mathrm{J} 0218+4232$ & $\mathrm{U}$ & 50.1 & 0.0 & 6.8 & $2.13 \pm 0.33$ & $2.72 \pm 0.26$ & \\
\hline $\mathrm{J} 0340+4130$ & M & 26.9 & 0.1 & 16.3 & $0.53 \pm 0.11$ & $0.02 \pm 0.22$ & $0.94 \pm 0.28$ \\
\hline $\mathrm{J} 1658-5324$ & $\mathrm{U}$ & 42.3 & 0.0 & 1.9 & $1.69 \pm 0.29$ & $2.52 \pm 0.76$ & \\
\hline $\mathrm{J} 2043+1711$ & $\mathrm{U}$ & 52.5 & 0.0 & 8.8 & $1.46 \pm 0.27$ & $2.29 \pm 0.14$ & \\
\hline $\mathrm{J} 2124-3358$ & M & 129. & 0.0 & 19.8 & $1.08 \pm 0.15$ & $0.70 \pm 0.51$ & $1.21 \pm 0.49$ \\
\hline $\mathrm{J} 2302+4442$ & M & 114. & 0.0 & 9.8 & $1.45 \pm 0.20$ & $1.54 \pm 0.40$ & $1.61 \pm 0.82$ \\
\hline
\end{tabular}

Notes. Off-peak regions with a significant detection of emission. The source classification is "M" for likely magnetospheric, "W" for likely pulsar wind, and "U" for unidentified. The table includes the significance of the source (TS), of the source extension (TS ext $_{\text {) }}$, and of a spectral cutoff ( $\mathrm{TS}_{\text {cut }}$ ). The best-fit energy flux and photon index are computed in the energy range from $100 \mathrm{MeV}$ to $316 \mathrm{GeV}$. Exponential cutoff energies are listed for sources with large $\mathrm{TS}_{\text {cut }}$. The quoted errors are statistical only. A few sources are discussed in Appendix C.

a The spectral shape of the Crab Nebula was taken from Buehler et al. (2012).

b The spectral shape of Vela-X was taken from Grondin et al. (2013).

Only one of the four, the previously identified Vela-X PWN (Abdo et al. 2010e), is spatially extended for the LAT.

We identify 19 type "U" regions, and 11 type "M" sources, significantly expanding the number of pulsars that perhaps have detectable magnetospheric emission across all rotational phases. One caution is that many of these "M" pulsars, especially the young objects, are in regions of particularly bright diffuse gamma-ray emission, where small fractional uncertainties in the level of diffuse emission can account for much of the apparent unpulsed emission. However, if established as true magnetospheric components, these will be important test cases for pulsar emission models. For type "M" and "U" sources, we present the best-fit spectral parameters using a point source at the pulsar's position with a PLEC1 spectral model in Table 12. For all other sources (except the Crab Nebula described in Section 7.2), we present the spectral results using a power-law spectral model and the best-fit spatial representation.
For a few sources, the spectral analysis performed here disagrees with that in Ackermann et al. (2011). For soft and faint sources (including J1044-5737 and J1809-2332), the spectral discrepancy is mainly caused by our use of a newer Galactic diffuse model. At lower energies, small changes in the diffuse model can have a significant impact on the analysis of a region. For bright magnetospheric sources (including J0633+1746 and $\mathrm{J} 2021+4026$ ), the spectral discrepancy is mainly due to using different phase ranges (see Section 7.1).

Figure 14 shows that only a small fraction of the spindown power goes into the gamma-ray emission from LAT-detected PWNe. Similarly, Figure 15 shows that the LAT only detects PWNe from the youngest pulsars with the highest spindown power. $\mathrm{GeV}$ emission from the Crab Nebula is highly time variable (Section 7.2). Indeed, we find $\mathrm{TS}_{\mathrm{var}}=373$ for the Crab Nebula; however no other source demonstrated flux variability (all have $16<\mathrm{TS}_{\mathrm{var}}<65$ ). Other GeV PWNe may be variable, 


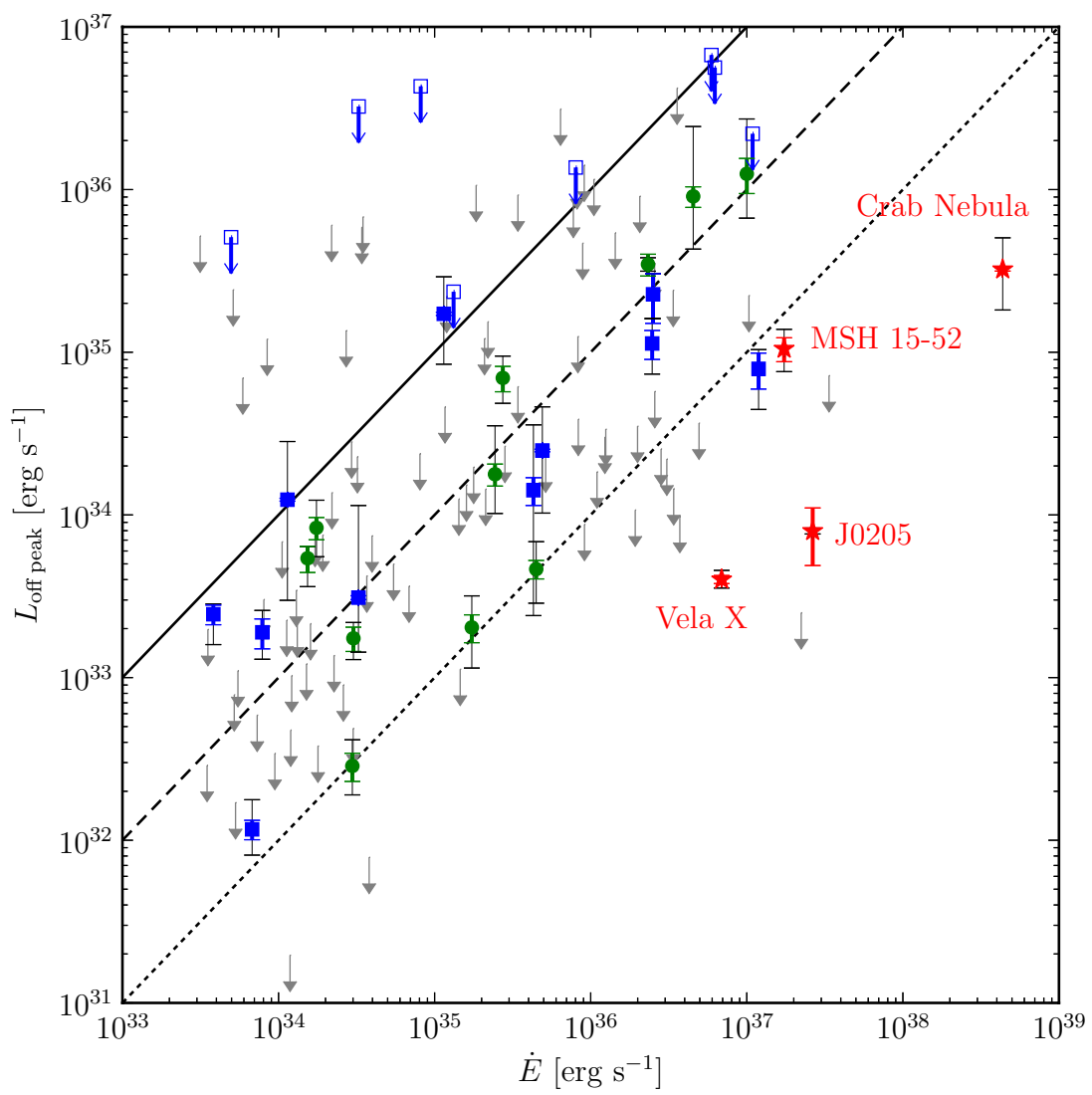

Figure 14. Off-peak luminosity compared to the observed pulsar spindown power. The luminosity is computed and plotted with the same convention as Figure 9. A luminosity upper limit is plotted when there is no significant off-peak emission or when there is only a distance upper limit. The star-shaped markers (colored red in the online version) represent type "W" sources, the square-shaped markers (colored blue) represent type "M" sources, circular markers (colored green) represent type "U" sources, and the gray arrows represent non-detections. The filled blue square-shaped markers represent "M" sources with a detected luminosity and the unfilled markers represent luminosity upper limits where there is only a distance upper limit. The solid, dashed, and dotted diagonals show $100 \%$, $10 \%$, and $1 \%$ efficiency (respectively).

(A color version of this figure is available in the online journal.)

but the combination of lower fluxes and less-extreme variations limits our ability to identify them as such.

The off-peak results for several interesting sources are presented in Appendix C. The complete off-peak search results can be found in the auxiliary information described in Appendix B. For regions where we find $\mathrm{TS}<25$, the auxiliary information contains upper limits computed for both a power-law spectral model and a PLEC1 model with $E_{\text {cut }}=3 \mathrm{GeV}$ and $\Gamma=1.7$. The auxiliary information also contains $\mathrm{TS}_{\mathrm{var}}$ for each off-peak interval.

\section{THE PULSARS NOT SEEN}

This catalog is a milestone in the progress toward the longterm goal of acquiring the most uniform sample of neutron stars possible, so that comparisons with model predictions (e.g., Gonthier et al. 2007; Watters \& Romani 2011; Pierbattista et al. 2012) will allow improved tests of emission models and of their links with their parent population of massive stars or with diffuse Galactic emission. Selection biases can be subtle and the advantage of pulsar searches in the coming years is not so much to increase the absolute numbers, but to be sure to have explored the dark corners of parameter space. Continued support from pulsar radio astronomers is crucial to maintain sensitivity to the more unusual gamma-ray pulsars in the coming years of the Fermi mission. Here, we consider pulsars that might have been expected to be seen with the LAT, but were not, to highlight "gamma-quiet" or "sub-luminous" pulsars.

\subsection{High Spindown Power Pulsars}

Of the 64 known RPPs with $\dot{E}>10^{36} \mathrm{erg} \mathrm{s}^{-1}$, Table 13 lists the 28 for which we did not see gamma-ray pulsations when the data set for this paper was frozen. When no steady LAT point source lies within 0.2 we provide a $95 \%$ confidence level upper limit (UL) on $G_{100}$ (Section 8.2). The Galactic latitude $b$ roughly indicates the diffuse background level. The last column compares the UL with the "heuristic" energy flux, $\sqrt{\dot{E}} /\left(4 \pi d^{2}\right)$. Figure 15 plots $\sqrt{\dot{E}} / d^{2}$ for all pulsars. Note the absence of the factor $4 \pi$ in the latter case. To convert the plot's scale, in units of $\operatorname{erg}^{1 / 2} \mathrm{~s}^{-1 / 2} \mathrm{kpc}^{-2}$, to the units used for $G_{100}$ and the flux ULs, using $L_{\gamma}^{h}$, (Equation (17)), we multiply the scale of Figure 15 by

$$
\begin{aligned}
& \frac{\sqrt{10^{33} \mathrm{erg} \mathrm{s}^{-1}}}{4 \pi\left(3.08 \times 10^{21} \mathrm{~cm} \mathrm{kpc}^{-1}\right)^{2}} \\
& =2.65 \times 10^{-28} \mathrm{erg}^{1 / 2} \mathrm{~s}^{-1 / 2} \mathrm{kpc}^{2} \mathrm{~cm}^{-2} .
\end{aligned}
$$

For several of the pulsars, the predicted flux is less than twice the UL. These pulsars seem to be near our current sensitivity limit, sensitive to distance, beaming, or local background 


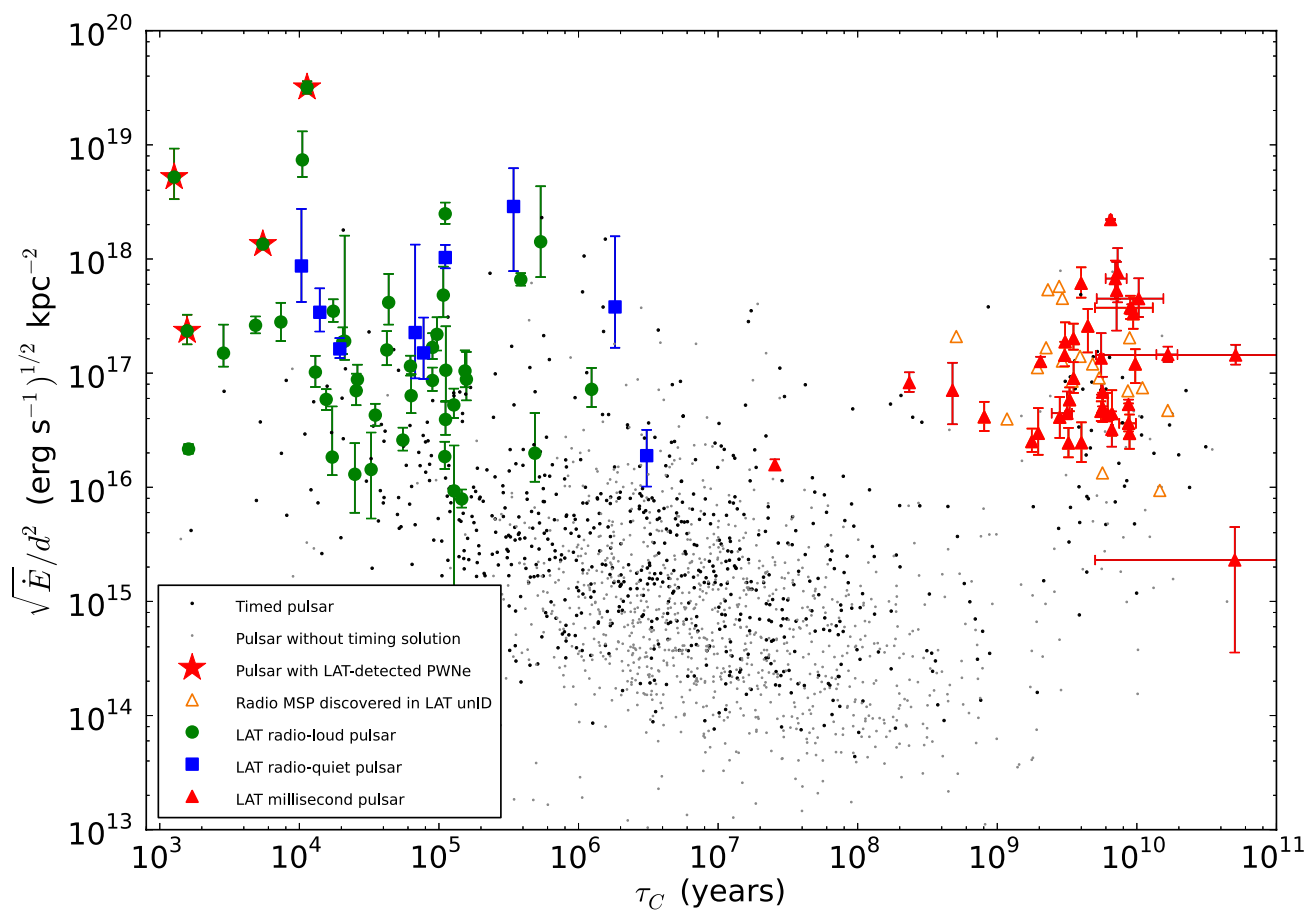

Figure 15. Figure-of-merit for the gamma-ray flux from a given pulsar, $\sqrt{\dot{E}} / d^{2}$, vs. the pulsar characteristic age $\tau_{c}$. For comparison with the integral energy flux $G_{100}$, a scale factor $2.65 \times 10^{-28}$ can be applied to the $y$-axis (see Section 8.1). The markers are the same as in Figure 1, with red stars added for the four pulsars associated with GeV PWNe (see Section 7). Black and gray dots include all $\dot{E}$ values, even though only high $\dot{E}$ pulsars have been seen in gamma rays to date.

(A color version of this figure is available in the online journal.)

uncertainties. We highlight a few pulsars from Table 13, in order of decreasing spindown power. The large distance $(54 \mathrm{kpc})$ to the Large Magellanic Cloud (LMC) is the simplest explanation for the non-detections of PSRs J0537-6910 and J0540-6919. Using highly accurate rotation ephemerides based on RXTE $\mathrm{X}$-ray observations and varied data selection cuts, we confirm the non-detection reported by Abdo et al. (2010k), with over three times as much data.

PSR J2022+3842 was thought to have the second-highest spindown power, after the Crab, of any known pulsar in the Milky Way (Arzoumanian et al. 2011). However, recent XMMNewton data revealed that the period is $48 \mathrm{~ms}$, twice the period previously seen with $R X T E$ and GBT, with a $4 \times$ smaller $\dot{E}$ value (Arumugasamy et al. 2013). Its DM distance is $10 \mathrm{kpc}$ but, situated in the heart of the Cygnus region, it (or PSR $\mathrm{J} 2021+3651)$ may be as close as $2 \mathrm{kpc}$. For $d=10 \mathrm{kpc}, \sqrt{\dot{E}} / d^{2}$ is $\approx 5 \times 10^{16} \mathrm{erg}^{1 / 2} \mathrm{~s}^{-1 / 2} \mathrm{kpc}^{-2}$. The LAT detects a source 0.06 away, with a spectrum adequately modeled by a power law with or without an exponential cutoff. The pulsar is difficult to detect in radio and suffers large timing noise; consequently, phase-folding the LAT data is difficult and blind period searches of the LAT data are hampered by the intense background. If unpulsed emission is confirmed, this could be an example of magnetospheric emission with low modulation and 100\% duty cycle. The second-highest spindown power now belongs to the radio-quiet, X-ray PSR J1813-1749. It has a highly uncertain distance, probably greater than $5 \mathrm{kpc}$ (Halpern et al. 2012), and is undetected with the LAT.

PSRs J1400-6325 and J1747-2809 are also distant, with slightly smaller $\sqrt{\dot{E}} / d^{2}$. The latter lies toward the Galactic center where the diffuse background level is intense and source density is high. The former is undetected in radio and was timed in X-rays; however, phase-connected ephemerides cover the
Fermi mission epoch only partially. The nearest source with $\mathrm{TS} \geqslant 25$ in an internal three-year source list, constructed in a similar fashion to 2FGL, is more than $1^{\circ}$ away. Curiously, Geminga and PSR J0007+7303 are the only X-ray loud, radioquiet pulsars detected by the LAT.

Both $\mathrm{J} 1617-5055$ and $\mathrm{J} 1930+1852$ are roughly in the same $\sqrt{\dot{E}} / d^{2} \approx 10^{17}$ erg $^{1 / 2} \mathrm{~s}^{-1 / 2} \mathrm{kpc}^{-2}$ range as the previous pulsars. J1617-5055 has particularly strong timing noise and the weekly to monthly Parkes observations did not allow a phase-connected timing solution covering the mission epoch. The rotation ephemeris was finally obtained in 2011 but gammaray phase-folding reveals no hint of pulsations (R. Shannon 2011, private communication). The nearest LAT source $(0.2$ away) is the PWN HESS J1616-508 (Lande et al. 2012). PSR $\mathrm{J} 1930+1852$ has an accurate radio ephemeris but no hint of gamma-ray pulsations. The nearest 2 FGL source with $\mathrm{TS} \geqslant 25$ is 0.55 away, nine times the $95 \%$ error radius for that source. Of the 14 pulsars for which $\mathrm{Ng} \&$ Romani (2008) fit the nebular torus seen in X-rays, PSR J1930+1852, in PWN G54.1 + 03 (Acero et al. 2013a), has the smallest angle between the spin axis and the line of sight. The radio beam intersects the Earth but the equatorial gamma-ray emission may not.

Skipping down the list, PSR J1928+1746 is within twice the $95 \%$ error radius of a source in an internal $3 \mathrm{yr}$ source list, but phase-folding provides no significant pulsations. Similar explorations for $10^{35}<\dot{E}<10^{36} \mathrm{erg} \mathrm{s}^{-1}$ led to the discovery of PSR J1913+0904 as a gamma-ray pulsar, listed in Table 4. Three pulsars for which the nearby high-TS LAT source is a gamma-ray pulsar are PSRs J1524-5625, J1803-2137, and J1831-0952. Searching the off-peak phase interval of the nearby pulsar reduces background but did not allow us to detect pulsations. PSR J1524-5625 bears special mention because of the large spread between the upper limit and the heuristic 
Table 13

Undetected RPPs with Spindown Power $\dot{E}>10^{36} \mathrm{erg} \mathrm{s}^{-1}$

\begin{tabular}{|c|c|c|c|c|c|}
\hline PSR & $\begin{array}{c}\dot{E} \\
\left(10^{36} \mathrm{erg} \mathrm{s}^{-1}\right)\end{array}$ & $\begin{array}{l}\text { Distance } \\
(\mathrm{kpc})\end{array}$ & $\begin{array}{l}b \\
\left(^{\circ}\right)\end{array}$ & $\begin{array}{c}\text { Flux UL } \mathrm{UL}^{\mathrm{a}} \\
\left(10^{-12} \mathrm{erg} \mathrm{cm}^{-2} \mathrm{~s}^{-1}\right)\end{array}$ & $\begin{array}{c}\sqrt{10^{33} \dot{E}} /\left(4 \pi d^{2}\right) \\
\left(10^{-12} \mathrm{erg} \mathrm{cm}^{-2} \mathrm{~s}^{-1}\right)\end{array}$ \\
\hline J0537-6910 & 488 & 53.7 & -31.7 & LMC & 2 \\
\hline J0540-6919 & 146 & 53.7 & -31.5 & LMC & 1 \\
\hline J1813-1749 & 56 & $>5^{\mathrm{b}}$ & -0.02 & $<26$ & $<80$ \\
\hline $\mathrm{J} 1400-6325$ & 51 & 11.3 & -1.6 & $<6$ & 15 \\
\hline $\mathrm{J} 1747-2809$ & 44 & 13.3 & 0.08 & $<15$ & 10 \\
\hline $\mathrm{J} 2022+3842$ & 30 & 10.0 & 0.96 & SNR G76.9 + 1.0 & 14 \\
\hline $\mathrm{J} 1617-5055$ & 16 & 6.8 & -0.28 & $\mathrm{PWN}^{\mathrm{c}}$ & 23 \\
\hline $\mathrm{J} 1930+1852$ & 12 & 7.0 & 0.27 & $<16$ & 18 \\
\hline J1849-0001 & 9.8 & 7.0 & 0.53 & $<15$ & 17 \\
\hline $\mathrm{J} 1846-0258$ & 8.1 & 5.8 & -0.24 & $<26$ & 22 \\
\hline J1811-1925 & 6.5 & $5^{\mathrm{b}}$ & -0.35 & $<24$ & 27 \\
\hline $\mathrm{J} 1838-0655$ & 5.6 & $\ldots$ & -0.20 & $\mathrm{UnId}^{\mathrm{c}}$ & $\ldots$ \\
\hline $\mathrm{J} 1856+0245$ & 4.6 & 9.0 & 0.06 & $<27$ & 7 \\
\hline $\mathrm{J} 1935+2025$ & 4.6 & 6.2 & -0.20 & $<18$ & 15 \\
\hline J1524-5625 & 3.2 & 2.8 & 0.35 & $<6$ & 61 \\
\hline $\mathrm{J} 1913+1011$ & 2.9 & 4.8 & -0.17 & $<23$ & 20 \\
\hline J1826-1334 & 2.8 & 3.9 & -0.69 & $\mathrm{PWN}^{\mathrm{c}}$ & 29 \\
\hline $\mathrm{J} 1803-2137$ & 2.2 & 4.4 & 0.15 & $\mathrm{~J} 1803.3-2148(110)$ & 20 \\
\hline $\mathrm{J} 1837-0604$ & 2.0 & 6.4 & 0.27 & $<31$ & 9 \\
\hline J1809-1917 & 1.8 & 3.5 & 0.08 & $<26$ & 28 \\
\hline $\mathrm{J} 1301-6305$ & 1.7 & 6.7 & -0.24 & $<14$ & 8 \\
\hline $\mathrm{J} 1614-5048$ & 1.6 & 7.9 & 0.17 & $<31$ & 5 \\
\hline $\mathrm{J} 1828-1101$ & 1.6 & 6.6 & 0.04 & $<23$ & 8 \\
\hline $\mathrm{J} 1928+1746$ & 1.6 & 5.8 & 0.11 & $\mathrm{~J} 1928.8+1740(33)$ & 10 \\
\hline $\mathrm{J} 1341-6220$ & 1.4 & 11.1 & -0.04 & $<18$ & 3 \\
\hline J1437-5959 & 1.4 & 8.1 & 0.23 & $<19$ & 5 \\
\hline J0855-4644 & 1.1 & $<0.9^{\mathrm{b}}$ & -1.0 & $<10$ & $>340$ \\
\hline $\mathrm{J} 1831-0952$ & 1.1 & 4.0 & -0.13 & $<17$ & 17 \\
\hline
\end{tabular}

Notes. Of the 64 known RPPs with spindown power $\dot{E}>10^{36} \mathrm{erg} \mathrm{s}^{-1}$, the above 28 were unpulsed in GeV gamma rays as this catalog was being prepared. Column 3 is distance (the ATNF database DIST1 parameter unless noted otherwise) and Column 4 is Galactic latitude. The upper limit in Column 5 is calculated using the all-sky model as described in Section 8.2. The heuristic spindown luminosity in Column 6 corresponds to the lower diagonal in Figure 9.

${ }^{a}$ An energy flux upper limit is given if the pulsar is not within 0.2 of a LAT 2FGL source; otherwise the source name is given, with the integral energy flux above $100 \mathrm{MeV}$ in parentheses. LMC is the Large Magellanic Cloud (Abdo et al. 2010k).

${ }^{\mathrm{b}}$ Distance constraint references: PSR J0855-4644 from Acero et al. (2013b); PSR J1811-1925 by association with SNR G11.2-0.3 (Tam \& Roberts 2003); PSR J1813-1749 from Halpern et al. (2012).

c The LAT detects a (possible) PWN extending to the pulsar position: HESS J1616-508, centered $\approx 0.2$ away (Lande et al. 2012); HESS J1825-137, centered $\approx 0.5$ away (Grondin et al. 2011); HESS J1837-069, centered $\approx 0.4$ away, may be a PWN powered by this pulsar (Lande et al. 2012).

energy flux. If the NE2001 DM distance is accurate this could be a gamma-quiet pulsar candidate. The same is also true for PSR J0855-4644, again with a distance caveat.

In 2FGL, 83 sources have a "PSR" identification and are in this catalog. An additional 27 sources have a "psr" association, meaning that the pulsar lies within the error ellipse of the source but $5 \sigma$ pulsations were not seen when 2 FGL was written. Since then, pulsations for 12 of the 27 have allowed firm identification, included in this catalog. Of the remaining 15, 12 are radio MSPs discovered at LAT source positions (Section 3.3). The remaining three spatial associations of young RPPs with 2FGL sources are PSRs J1632-4818, J1717-5800, and J1928+1746. The last was discussed above. PSR J1632-4818 is a typical gamma-ray candidate $\left(\dot{E}=4.8 \times 10^{34} \mathrm{erg} \mathrm{s}^{-1}, d \approx 8 \mathrm{kpc}, b=-0.21\right)$ except that it has one of the highest surface B-fields of any RPP. It shows no hint of pulsations and the 2FGL association could be a chance spatial coincidence, or PSR J1632-4818 may be a candidate for unpulsed magnetospheric emission. PSR J1717-5800 is almost certainly a chance spatial coincidence: it is well below the empirical deathline $(\dot{E}=2.3 \times$ $\left.10^{32} \mathrm{erg} \mathrm{s}^{-1}\right)$ and not nearby $(d \approx 3.5 \mathrm{kpc})$, although with low background so far from the Galactic plane $(b=-11.5)$. Folding with an archival ephemeris showed no hint of pulsations.

Figure 15 illustrates the utility of the commonly used detectability metric $\sqrt{\dot{E}} / d^{2}$, showing an approximate LAT threshold of $\sim 10^{16} \mathrm{erg}^{1 / 2} \mathrm{~s}^{-1 / 2} \mathrm{kpc}^{-2}$. While the difficulty of establishing reliable distances for many pulsars (Section 4.2) makes quantitative comparison challenging, it is clear that this is a good predictor of pulsar detectability. However, as emphasized by Romani et al. (2011) there are a number of pulsars with a high detectability metric not seen by the LAT (Section 8), indicated by black dots above the LAT threshold in Figure 15. In some cases, underestimated distances may explain the non-detections; however, in other cases the pulsars have accurate parallax measurements and the gamma-ray beam must either miss Earth (e.g., a pulsar viewed at small $\zeta$ for outer-magnetosphere models) or have light curves with very small modulation amplitude (e.g., a large, unpulsed component for SG or aligned polar cap models). We note that in many cases no LAT flux is detected in the pulsar direction, so beaming presents the most likely explanation. 
In summary: many non-detections are due to large distances and/or background. In a few rare cases, our rotation ephemeris allows inadequate phase-folding. However, we are also accumulating a sample of "gamma-quiet" pulsars as well as a sample of possibly unpulsed gamma-ray pulsars, that is, for which the emission from the magnetosphere is unmodulated.

\subsection{Flux Upper Limits and Sensitivity}

Figure 16 maps the LAT sensitivity on the sky for the phase-averaged detection of a point source with a pulsar-like spectrum, for the three-year data set. To build the map, we started from the all-sky source model. For each point on a 0.15 grid we added an additional pulsar-like point source with fixed parameters of $\Gamma=1.8$ and $E_{\text {cut }}=2 \mathrm{GeV}$ for the PLEC1 spectral shape (see Section 6) and re-fit the data. We then use the corresponding likelihood, as a function of the flux, to determine 95\% confidence-level upper limits. This underestimates the actual sensitivity for two reasons. First, for weak sources near the threshold, leaving $\Gamma$ and $E_{\text {cut }}$ as free parameters during the data fitting increases the likelihood function peak width by a factor of two. Second, we claim a point-source detection only if the signal can be localized. To account for these effects, we increased the derived flux limit by a factor of two. The result is consistent with the measured fluxes of detected sources. The apparent fluctuations along the Galactic plane are a consequence of the limitations of the interstellar emission model used to represent the diffuse background. Discontinuities result from different optimized normalizations for the ROIs selected for the all-sky analysis that were used to calculate the sensitivity limits. The upper limits in Table 13 correspond to the values nearest the positions of those pulsars.

Figure 17 shows the LAT pulsars' integral energy flux from 0.1 to $100 \mathrm{GeV}\left(G_{100}\right.$; see Section 6$)$, versus their Galactic latitude $b$. Also shown is the latitude dependence of the sensitivity from Figure 16, averaged over longitude, with the $10 \%$ and $90 \%$ percentile limits. The minimum detectable flux increases with the background level, causing a selection bias against low-latitude pulsars seen against the bright Galactic background. Below $20 \times 10^{-12} \mathrm{erg} \mathrm{cm}^{-2} \mathrm{~s}^{-1}$ all but one pulsar was discovered in gamma rays by phase-folding using rotation ephemerides obtained from radio or X-ray data. The gamma-selected pulsars, those discovered in a blind period search of LAT data, are brighter in gamma rays. Dormody et al. (2011) found that the blind-search sensitivity is a factor of 2.5 worse than for searches using ephemerides. The nine pulsars directly on the plane, and/or with very low fluxes, having phase-averaged significances below the formal detection threshold (TS $<25$ ) were all found with ephemerides. The observed minimum energy fluxes for LAT pulsars are below $5 \times 10^{-12} \mathrm{erg} \mathrm{cm} \mathrm{cm}^{-2} \mathrm{~s}^{-1}$ far from the Galactic plane. PSR $\mathrm{J} 2240+5832(l, b=106.6,-0.11)$ is an example of a pulsar with measured flux right at the sensitivity threshold. Figure 22(h) shows that it has particularly narrow peaks, facilitating its pulsed detection without improving its phase-averaged significance. Pulsars with spectral parameters far from the average values assumed for the sensitivity sky map also lead to outlying points.

\subsection{SNRs and PWNe without Detected Gamma-ray Pulsars}

Table 14 compiles pulsars explored in Fermi-LAT studies of 19 SNRs. Eleven of the SNRs are spatially extended at GeV energies. Of these, seven have known CCOs: IC 443, Puppis A, RX J1713.7-3946, S147, W30, W44, and W28. The remaining four (Cygnus Loop, HB21, RX J0852.0-4622 and W51C) have associated CCOs, or candidate PWNe indicating the likely presence of a CCO. Their gamma-ray emission is more consistent with a single extended source than a composite system of a pulsar and a remnant. Three of the remaining GeV SNRs (Cassiopeia A, Tycho and W49B) show no evidence for extended emission at $\mathrm{GeV}$ or $\mathrm{TeV}$ energies. The final five SNRs (3C 58, Crab, Vela, MSH 15-52, and MSH 11-62) contain gamma-ray pulsars. MSH 11-62 has no off-peak detection, meaning the gamma-ray emission is completely due to nearby PSR J1105-6107 with no detected SNR contribution.

\subsection{Toward TeV Energies}

Both the MAGIC and VERITAS atmospheric Cherenkov telescopes (ACTs) detected pulsations from the Crab, with an integral photon flux above $100 \mathrm{GeV}$ of $\sim 6 \times 10^{-12} \mathrm{~cm}^{-2} \mathrm{~s}^{-1}$ (Aliu et al. 2008, 2011; Aleksić et al. 2012). Extrapolating the LAT Crab pulsar spectrum (Table 9) predicts an integral flux above $100 \mathrm{GeV}$ of less than $10^{-19} \mathrm{~cm}^{-2} \mathrm{~s}^{-1}$ for a power law with a pure exponential cutoff ( $b=1$ in Equation (12)). Fitting the cutoff shape yields $b=0.44$ and the extrapolation again yields a value far below their measurement. In consequence these authors fit the joint LAT-ACT data with a broken power law, bridging any dip that may exist between the LAT and ACT energy ranges. We have examined whether the high-energy tails of the LAT data point to other pulsars that may be detectable by ground-based instruments, or that may help distinguish between the various emission models.

For our brightest and hardest pulsars (some LAT events aligned in position and phase with Vela's second gamma-ray peak exceed $50 \mathrm{GeV}$ ) we fit the LAT data to a broken power law. We also simulated three-year data sets using the standard LAT tool gtobssim and fit the simulated data in the same way. We find that the extrapolations to ACT energies are unreliable. The main problem is that the few gamma rays with the highest energies greatly influence the spectral parameters. The fits are also sensitive to the low-energy bound of the data set. Varying the functional form further broadens the range of extrapolated fluxes, predicting anything from quick to extremely difficult detections by ground-based instruments. We chose to make no such predictions here and advise caution in extrapolating the $\mathrm{GeV}$ data. The LAT collaboration is currently preparing a catalog of sources detected above $10 \mathrm{GeV}$ (The FermiLAT Collaboration 2013). Of the 27 sources associated with known pulsars, 20 (11) have significant pulsations in the range $>10 \mathrm{GeV}(>25 \mathrm{GeV})$.

\section{MULTIWAVELENGTH COUNTERPARTS}

\subsection{X-Ray Properties}

Gamma-ray pulsars are usually observed to release most of their pulsed energy in the $\mathrm{GeV}$ range, but they are inherently multiwavelength objects. X-ray emission associated with individual pulsars is often detected with a significance higher than $5 \sigma$. Extensive work on X-ray pulsars was enabled by ROSAT and $A S C A$ (Becker \& Truemper 1997). The X-ray flux can be pulsed non-thermal emission from the magnetosphere; blackbody thermal emission from the neutron star surface, either pulsed or unpulsed; or extended emission from a PWN energized by particles accelerated by the pulsar. Information from $\mathrm{X}$-ray observations should be included in any audit of the rotational energy loss of a given pulsar. 


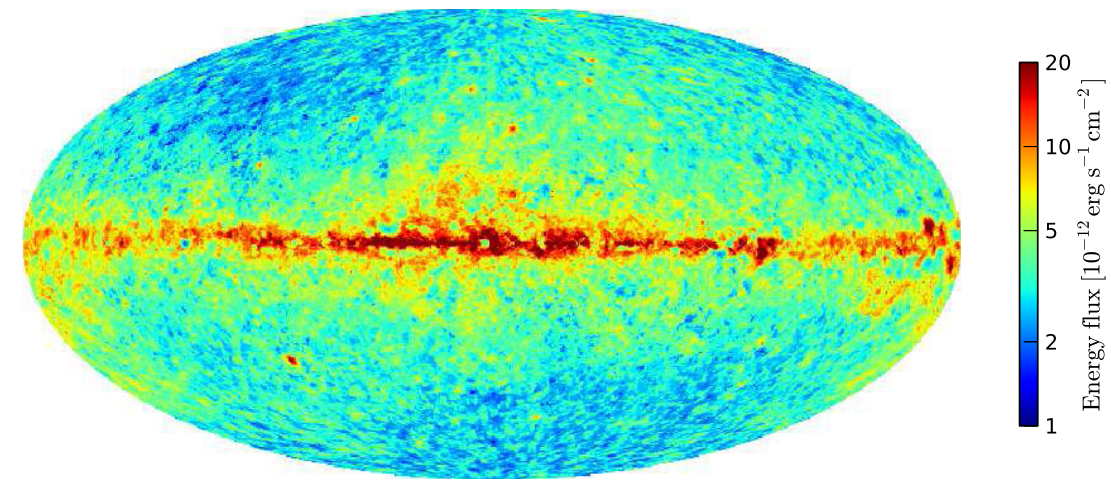

Figure 16. Hammer-Aitoff projection of the LAT three-year sky-survey energy flux sensitivity above $100 \mathrm{MeV}$, assuming a pulsar-like exponentially cutoff power law energy spectrum.

(A color version of this figure is available in the online journal.)

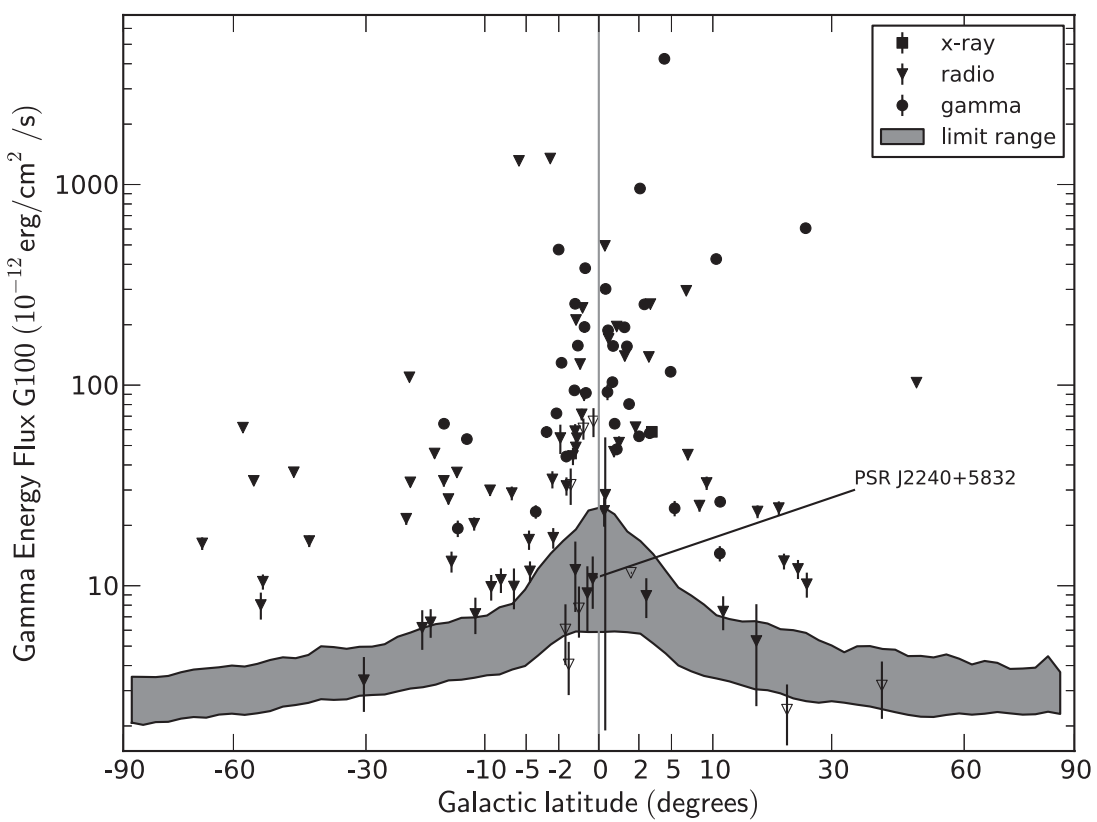

Figure 17. Integral energy flux from 0.1 to $100 \mathrm{GeV}, G_{100}$, vs. Galactic latitude $b$ (scaled as $b^{0.65}$ for clarity). Circles indicate gamma-selected pulsars discovered in blind period searches, while triangles and squares indicate previously known pulsars discovered in gamma rays by phase-folding with rotation ephemerides obtained from radio or X-ray data. Open symbols indicate TS $<25$. The gray band shows the $10 \%$ to $90 \%$ percentile range of the three-year mean sensitivity for point-source detection averaged over longitude.

To characterize the X-ray spectra of LAT-detected pulsars, we use only photons with energies from 0.3 to $10 \mathrm{keV}$ collected by any of the major contemporary observatories operating in the soft X-ray band: Chandra/ACIS (Garmire et al. 2003), XMM-Newton (Strüder et al. 2001; Turner et al. 2001), Swift/ XRT (Burrows et al. 2005), and Suzaku (Mitsuda et al. 2007). Unlike in the gamma-ray band, the X-ray coverage of LAT pulsars is uneven since the majority of the newly discovered pulsars (which account for half of the entries in the present catalog) have never been the targets of deep X-ray observations, while for other well-known gamma-ray pulsars, such as Crab, Vela, and Geminga extensive observations have been carried out. However, all LAT pulsars do have some degree of X-ray coverage, ranging from few-ks shallow snapshots with Swift/ XRT to orbit-long, deep observations by Chandra, XMMNewton or Suzaku.

Tables 15 and 16 compile X-ray spectral results for all pulsars in this catalog. Given the complex phenomenology of pulsar $\mathrm{X}$-ray emission, we have attempted to categorize the fluxes in a manner that will support comparisons and statistical studies focused on the system energy audit.

The status and quality for X-ray detections are indicated as follows: " 0 " indicates no confirmed X-ray counterpart (or a purely thermal emission without a non-thermal spectral component), " 1 " indicates that a counterpart has been identified but with too few counts for further characterization, and "2" indicates sufficient information for spectral characterization (e.g., Ray et al. 2011; Abdo et al. 2012). An ad hoc analysis was performed for some pulsars for which the standard analysis could not be applied (e.g., owing to the very intense thermal component of the spectrum of Vela or to an active galaxy near PSR J1418-6058); we designate them as type " $2 *$ " pulsars.

We consider an X-ray counterpart to be established if (1) $\mathrm{X}$-ray pulsations have been detected, (2) X-ray and radio pulsar positions coincide, or (3) LAT timing (Ray et al. 2011) yields a position good enough to claim a high confidence identification with an X-ray source. The probability of finding a serendipitous source located inside a typical Chandra error circle is less than 
Table 14

GeV-detected SNRs with Dedicated Studies

\begin{tabular}{|c|c|c|c|c|c|c|}
\hline SNR Name & Common Name(s) & Objects within Region of Interest & $\begin{array}{c}\text { Gamma-ray } \\
\text { Pulsar? }\end{array}$ & $\begin{array}{c}\text { Emission } \\
\text { Source }\end{array}$ & Method & Ref. \\
\hline SNR 120.1+01.4 & Tycho, 3C 10, SN 1572 & None & $\ldots$ & remnant only & spectral & 15 \\
\hline SNR $130.7+03.1$ & $3 \mathrm{C} 58, \mathrm{SN} 1181$ & PSR J0205+6449 $a$ & $\mathrm{Y}$ & composite & off-pulse & 1 \\
\hline SNR 184.6-05.8 & Crab & PSR J0534+2200 $a$ & $\mathrm{Y}$ & composite & off-pulse & 3 \\
\hline SNR 180.0-01.7 & S147 & PSR J0538+2817 & $\mathrm{N}$ & remnant only & spatial & 18 \\
\hline SNR 189.1+03.0 & IC443 & PWN CXOU J061705.3+222127 & $\ldots$ & remnant only & spectral & 5 \\
\hline SNR 260.4-03.4 & Puppis A & PSR J0821-4300 $a$ & $\ldots$ & remnant only & spatial & 20 \\
\hline SNR 263.9-03.3 & Vela & PSR J0831-4406, PSR J0835-4510 $a$, PWN Vela-X & $\mathrm{Y}$ & composite & off-pulse & 6 \\
\hline SNR 266.2-01.2 & Vela Jr. & PSR J0855-4644, PSR J0855-4658 & $\mathrm{N}$ & remnant only & spectral & 12 \\
\hline SNR 291.0-00.1 & MSH 11-62 & PSR J1105-6107 & $\mathrm{Y}$ & pulsar only & off-pulse & 17 \\
\hline SNR 320.4-01.2 & MSH $15-52$ & PSR J1513-5908 $a$ & $\mathrm{Y}$ & composite & off-pulse & 7 \\
\hline SNR 347.3-00.5 & RX J1713.7-3946 & PSR J1712-391, J1712-392, J1713-3949 & $\ldots$ & remnant only & spectral & 11 \\
\hline SNR 006.4-00.1 & W28 & PSR J1759-2307, PSR J1800-2343 & $\mathrm{N}$ & remnant only & spatial & 8 \\
\hline SNR 008.7-00.1 & W30 & PSR J1803-2137 $a$, PSR J1806-2125 & $\mathrm{N}$ & remnant only & spatial & 14 \\
\hline SNR 034.7-00.4 & W44 & PSR J1856+0113 $a$ & $\mathrm{~N}$ & remnant only & spatial & 10,16 \\
\hline SNR 043.3-00.2 & W49B & None & $\mathrm{N}$ & remnant only & spectral & 9 \\
\hline SNR 049.2-00.7 & W51c & PWN CXOU J192318.5+140335 & $\mathrm{N}$ & remnant only & spatial & 2 \\
\hline SNR 074.0-08.5 & Cygnus Loop & PWN 2XMM J204920.2+290106 & $\ldots$ & remnant only & spatial & 13,19 \\
\hline SNR 089.0+04.7 & HB21 & None & $\ldots$ & remnant only & spatial & 21 \\
\hline SNR 111.7-02.1 & Cassiopeia A & $\mathrm{CCO}$ & $\mathrm{N}$ & remnant only & spectral & 4 \\
\hline
\end{tabular}

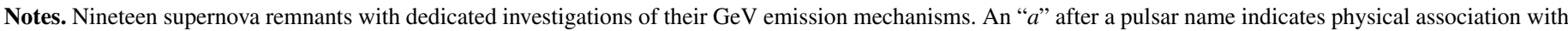
the SNR. Four of the five gamma-ray pulsars ("Y" in Column 4) are associated with SNRs, while PSR J1105-6107 merely overlaps.

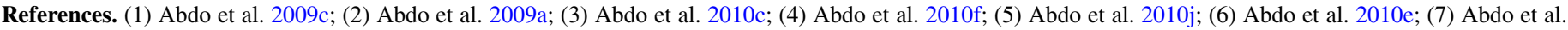

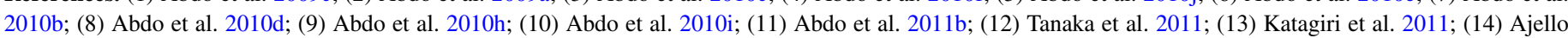

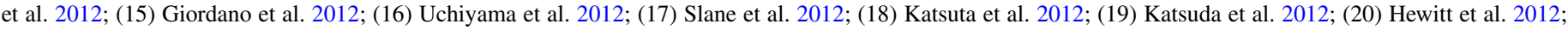
(21) Reichardt et al. 2012.

0.0005 (Ebisawa et al. 2005; Novara et al. 2006); however, the probability increases by a factor of $\sim 50$ for $S u z a k u$ observations owing to a more-limited spatial resolution. Thus, we label all the objects found by Suzaku as " 1 *" to indicate that there is a nonnegligible possibility of a chance coincidence. All the pulsar and nebular spectra have been modeled as absorbed power laws. Blackbody components have been added to the spectra when statistically needed. Absorption along the line of sight has been evaluated through the fitting procedure. However, for pulsars with very low statistics we used values derived from observations taken in different bands, when available. We note that six MSPs can be fitted only with a thermal model: thus, owing to the lack of any non-thermal component, we designate them as "type 0." According to our classification scheme, we have 50 type 0,11 type 1 , and 56 type 2 pulsars. In total, 67 gamma-ray pulsars (30 radio-loud, 19 radio-quiet, and 18 millisecond) have an X-ray counterpart with a non-thermal spectral component.

For each type 2 pulsar, we checked for a possible PWN contribution. We analyzed all the data to search for extended emission through a radial brilliance study. When a PWN was found, its contribution was evaluated as follows: we extracted photons from an inner circular region containing 95\% of the point-like source counts, following the prescriptions suggested for each telescope. Such a region contains both the PSR and the PWN so that its X-ray flux must be fitted with two absorbed (PWN and PSR) power laws (plus a blackbody, if needed). We also selected an ad hoc outer region containing the brightest part of the nebula and fitted it with a single (PWN) power law, forcing the $N_{\mathrm{H}}$ and the PWN photon index values to be identical for the two (inner and outer) spectra. The PWN fluxes listed in Tables 15 and 16 are the spatially integrated fluxes for the two regions. Details on data analysis and fitting procedures for each telescope can be found in Marelli et al. (2011) and Marelli (2012).

For pulsars with a confirmed counterpart but too few photons to distinguish the spectral shape (type "1") the unabsorbed flux is estimated assuming a single power-law spectrum with photon index 2 to characterize all components, an absorbing column obtained by rescaling the Galactic column density estimated from the Leiden-Argentine-Bonn Survey of Galactic H I (Kalberla et al. 2005), ${ }^{101}$ and the distances from Tables 5 and 6. For type 1 pulsars we assume that the combined PWN and PSR thermal contributions account for $30 \%$ of the total source flux, a value comparable to the mean value obtained for type 2 objects. For pulsars without a confirmed counterpart (type " 0 ") an upper limit is shown, again derived assuming a photon index of 2 and a signal-to-noise ratio of 3 .

A plot $G_{100} / F_{X}$ as a function of $\dot{E}$ (see Figure 18) for type 2 pulsars shows a three-decade spread in the $G_{100} / F_{X}$ values for a given value of $\dot{E}$. This lack of correlation between gammaray and non-thermal X-ray fluxes may point to important (yet poorly-understood) differences in the geometry and/or height of the $\mathrm{X}$ - and gamma-ray emitting regions within the magnetosphere.

In general, radio-quiet pulsars are characterized by fainter $\mathrm{X}$-ray counterparts than radio-loud pulsars. Indeed, the X-ray fluxes of LAT-discovered radio-quiet population have less scatter than the radio-loud pulsars. MSPs have the lowest gammato-X flux ratio with less apparent scatter than that observed in young pulsars. These results confirm and expand those obtained by Marelli et al. (2011) and Marelli (2012) for smaller samples of LAT pulsars.

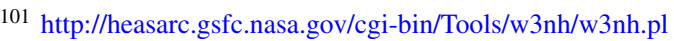


Table 15

X-Ray Spectral Parameters of Young LAT-detected Pulsars and Their Nebulae

\begin{tabular}{|c|c|c|c|c|c|c|}
\hline $\mathrm{PSR}^{\mathrm{a}}$ & $\mathrm{Inst}^{\mathrm{b}}$ & $\begin{array}{c}N_{\mathrm{H}} \\
\left(10^{20} \mathrm{~cm}^{-2}\right)\end{array}$ & $\begin{array}{c}F_{X}^{\mathrm{nt}} \\
\left(10^{-13} \mathrm{erg} \mathrm{cm}^{-2} \mathrm{~s}^{-1}\right)\end{array}$ & $\begin{array}{l}\text { Sp. Type, } \\
\text { Pulsed }^{\text {d }}\end{array}$ & $G_{100} / F_{X}^{\mathrm{nt}}$ & $\begin{array}{c}F_{X}^{\mathrm{pwn}} \\
\left(10^{-13} \mathrm{erg} \mathrm{cm}^{-2} \mathrm{~s}^{-1}\right)\end{array}$ \\
\hline $\mathrm{J} 0007+7303^{2}$ & $\mathrm{X}+\mathrm{C}$ & $16.6_{-7.6}^{+8.9}$ & $0.98 \pm 0.01$ & BB+Pow, $\mathrm{P}$ & $4320 \pm 70$ & $21.40_{-0.17}^{+0.14}$ \\
\hline $\mathrm{J} 0106+4855^{0}$ & $\mathrm{Su}$ & $10^{\mathrm{c}}$ & $<0.84$ & $\ldots$ & $>229$ & $\ldots$ \\
\hline $\mathrm{J} 0205+6449^{2}$ & $\mathrm{C}$ & $45.0_{-1.1}^{+1.3}$ & $19.70 \pm 0.70$ & $\mathrm{BB}+$ Pow, $\mathrm{P}$ & $29.7 \pm 2.1$ & $24.0 \pm 0.5$ \\
\hline $\mathrm{J} 0248+6021^{0}$ & $\mathrm{~S}$ & $80^{c}$ & $<9.00$ & $\ldots$ & $>57.4$ & 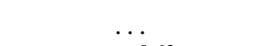 \\
\hline $\mathrm{J} 0357+3205^{2}$ & $\mathrm{C}$ & $8.0 \pm 4.0$ & $0.64_{-0.06}^{+0.09}$ & Pow, P & $1000_{-100}^{+150}$ & $3.72_{-1.36}^{+0.62}$ \\
\hline $\mathrm{J} 0534+2200^{2}$ & $\mathrm{~L}$ & $34.5 \pm 0.2$ & $44300 \pm 1000$ & Pow, P & $0.296 \pm 0.007$ & $396000 \pm 1000$ \\
\hline $\mathrm{J} 0622+3749^{0}$ & $\mathrm{C}$ & $10^{\mathrm{c}}$ & $<2.58$ & $\ldots$ & $>56.1$ & $\ldots$ \\
\hline $\mathrm{J} 0631+1036^{0}$ & $\mathrm{X}$ & $20^{\mathrm{c}}$ & $<0.23$ & $\ldots$ & $>2070$ & $\ldots$ \\
\hline $\mathrm{J} 0633+0632^{2}$ & $\mathrm{C}$ & $6.08_{-6.08}^{+21.91}$ & $0.63 \pm 0.05$ & $\mathrm{BB}+\mathrm{Pow}$ & $1510 \pm 170$ & $2.92_{-0.81}^{+0.79}$ \\
\hline $\mathrm{J} 0633+1746^{2}$ & $\mathrm{~L}$ & $1.07^{\mathrm{c}}$ & $4.97_{-0.27}^{+0.09}$ & $\mathrm{BB}+\mathrm{Pow}, \mathrm{P}$ & $8520_{-460}^{+160}$ & $0.172 \pm 0.001$ \\
\hline $\mathrm{J} 0659+1414^{2}$ & $\mathrm{~L}$ & $4.3 \pm 0.2$ & $4.06_{-0.59}^{+0.03}$ & BB+Pow, P & $61.8_{-10.9}^{+6.3}$ & $\mathrm{~N}$ \\
\hline $\mathrm{J} 0729-1448^{0}$ & $\mathrm{C}$ & $50^{\mathrm{c}}$ & $<0.37$ & $\ldots$ & $>318$ & $\ldots$ \\
\hline $\mathrm{J} 0734-1559^{0}$ & S & $20^{\mathrm{c}}$ & $<2.36$ & $\ldots$ & $>236$ & $\ldots$ \\
\hline $\mathrm{J} 0742-2822^{0}$ & $\mathrm{X}$ & $20^{\mathrm{c}}$ & $<0.23$ & $\ldots$ & $>771$ & $\ldots$ \\
\hline $\mathrm{J} 0835-4510^{2 *}$ & $\mathrm{~L}$ & $2.2 \pm 0.5$ & $65.1 \pm 15.7$ & $\mathrm{BB}+$ Pow, $\mathrm{P}$ & $1410 \pm 340$ & $128 \pm 1$ \\
\hline J0908-4913 & $\mathrm{C}+\mathrm{X}$ & $80^{c}$ & $<0.39$ & $\ldots$ & $>1130$ & $\ldots$ \\
\hline $\mathrm{J} 0940-5428^{0}$ & $\mathrm{C}$ & $50^{\mathrm{c}}$ & $<0.13$ & $\ldots$ & $>314$ & $\ldots$ \\
\hline $\mathrm{J} 1016-5857^{2}$ & $\mathrm{C}$ & $57.5_{-19.5}^{+23.5}$ & $1.47_{-1.31}^{+0.40}$ & Pow & $370_{-343}^{+137}$ & $3.53_{-2.77}^{+0.26}$ \\
\hline $\mathrm{J} 1019-5749^{0}$ & S & $150^{\mathrm{c}}$ & $<1.50$ & $\ldots$ & $>51.4$ & \\
\hline $\mathrm{J} 1023-5746^{2 *}$ & $\mathrm{C}$ & $117_{-33}^{+37}$ & $0.94_{-0.60}^{+0.19}$ & Pow & $2070_{-1320}^{+460}$ & $0.853_{-0.593}^{+0.193}$ \\
\hline $\mathrm{J} 1028-5819^{1}$ & $\mathrm{C}+\mathrm{Su}$ & $<15.0$ & $0.45 \pm 0.13$ & Pow & $5390 \pm 1660$ & $\ldots$ \\
\hline $\mathrm{J} 1044-5737^{1 *}$ & $\mathrm{Su}$ & $<12.9$ & $0.92_{-0.59}^{+0.26}$ & Pow & $1700_{-1090}^{+490}$ & $\ldots$ \\
\hline $\mathrm{J} 1048-5832^{2 *}$ & $\mathrm{C}+\mathrm{X}$ & $46.0 \pm 2.3$ & $0.49_{-0.34}^{+0.18}$ & Pow & $4000_{-2800}^{+1490}$ & $0.608_{-0.426}^{+0.224}$ \\
\hline $\mathrm{J} 1057-5226^{2}$ & $\mathrm{C}+\mathrm{X}$ & $2.7 \pm 0.2$ & $1.51_{-0.13}^{+0.02}$ & $\mathrm{BB}+$ Pow, $\mathrm{P}$ & $1950_{-170}^{+40}$ & $\mathrm{~N}$ \\
\hline $\mathrm{J} 1105-6107^{0}$ & $\mathrm{C}$ & 50 & $<0.08$ & $\ldots$ & $>6130$ & $\ldots$ \\
\hline $\mathrm{J} 1112-6103^{2}$ & $\mathrm{C}$ & $121_{-50}^{+76}$ & $0.57 \pm 0.28$ & Pow & $1070 \pm 560$ & $0.63 \pm 0.24$ \\
\hline $\mathrm{J} 1119-6127^{2}$ & $\mathrm{C}+\mathrm{X}$ & $185_{-38}^{+42}$ & $1.48 \pm 0.21$ & BB+Pow, P & $483 \pm 84$ & $0.601 \pm 0.194$ \\
\hline J1124-5916 & $\mathrm{C}$ & $30.0_{-4.8}^{+2.8}$ & $9.78_{-1.08}^{+1.18}$ & BB+Pow, $\mathrm{P}$ & $63.1_{-9.0}^{+9.5}$ & $5.17_{-0.30}^{+0.24}$ \\
\hline $\mathrm{J} 1135-6055^{2}$ & $\mathrm{C}$ & $41.9_{-15.2}^{+18.9}$ & $0.37_{-0.32}^{+0.15}$ & Pow & $1290_{-1130}^{+520}$ & $1.87_{-1.05}^{+0.39}$ \\
\hline $\mathrm{J} 1357-6429^{2}$ & $\mathrm{X}$ & $18.9_{-4.5}^{+4.8}$ & $0.42 \pm 0.16$ & $\mathrm{BB}+$ Pow, $\mathrm{P}$ & $809 \pm 324$ & $3.81_{-0.52}^{+0.38}$ \\
\hline $\mathrm{J} 1410-6132^{0}$ & S & $200^{c}$ & $<1.80$ & $\ldots$ & $>366$ & $\ldots$ \\
\hline $\mathrm{J} 1413-6205^{1 *}$ & $\mathrm{C}+\mathrm{Su}$ & $40^{c}$ & $1.41 \pm 0.38$ & Pow & $1120 \pm 310$ & $\ldots$ \\
\hline $\mathrm{J} 1418-6058^{2}$ & $\mathrm{C}+\mathrm{X}$ & $225_{-45}^{+52}$ & $0.36 \pm 0.14$ & Pow & $8400 \pm 3420$ & $\mathrm{Y}$ \\
\hline $\mathrm{J} 1420-6048^{2 *}$ & $\mathrm{X}$ & $202_{-106}^{+161}$ & $1.60 \pm 0.70$ & Pow & $1060 \pm 480$ & $\ldots$ \\
\hline $\mathrm{J} 1429-5911^{0}$ & $\mathrm{Su}$ & $80^{\mathrm{c}}$ & $<0.73$ & $\ldots$ & $>1100$ & $\ldots$ \\
\hline $\mathrm{J} 1459-6053^{2}$ & $\mathrm{X}$ & $42.0_{-18.0}^{+47.0}$ & $0.85 \pm 0.23$ & Pow & $1520 \pm 420$ & $\mathrm{~N}$ \\
\hline $\mathrm{J} 1509-5850^{2}$ & $\mathrm{C}+\mathrm{X}$ & $79.5_{-16.5}^{+22.1}$ & $0.53_{-0.18}^{+0.20}$ & Pow & $2380_{-830}^{+900}$ & $2.47_{-0.54}^{+0.32}$ \\
\hline $\mathrm{J} 1513-5908^{2 *}$ & $\mathrm{C}+\mathrm{L}^{f}$ & $91.8 \pm 0.2$ & $52 \pm 18$ & Pow & $0.612 \pm 0.284$ & $1459.5_{-12.7}^{+12.9}$ \\
\hline $\mathrm{J} 1531-5610^{1}$ & $\mathrm{C}$ & $40^{\mathrm{c}}$ & $1.62 \pm 0.53$ & Pow & $\ldots$ & $\ldots$ \\
\hline $\mathrm{J} 1620-4927^{0}$ & $\mathrm{X}$ & $40^{c}$ & $<0.67$ & $\ldots$ & $>2330$ & $\ldots$ \\
\hline $\mathrm{J} 1648-4611^{0}$ & $\mathrm{C}$ & $100^{c}$ & $<0.22$ & $\ldots$ & $>2520$ & $\ldots$ \\
\hline $\mathrm{J} 1702-4128^{1}$ & $\mathrm{C}+\mathrm{X}$ & $100^{c}$ & $0.23 \pm 0.07$ & Pow & $3150_{-3150}^{+4500}$ & $\ldots$ \\
\hline $\mathrm{J} 1709-4429^{2}$ & $\mathrm{C}+\mathrm{X}$ & $45.6_{-2.9}^{+4.4}$ & $3.78_{-0.94}^{+0.37}$ & $\mathrm{BB}+$ Pow, $\mathrm{P}$ & $3560_{-890}^{+350}$ & $8.36_{-0.67}^{+0.52}$ \\
\hline $\mathrm{J} 1718-3825^{2}$ & $\mathrm{X}$ & $40.7_{-15.5}^{+14.6}$ & $1.18_{-0.97}^{+0.58}$ & Pow & $753_{-622}^{+375}$ & $1.33_{-0.95}^{+0.55}$ \\
\hline $\mathrm{J} 1730-3350^{\circ}$ & $\mathrm{C}+\mathrm{X}$ & $100^{c}$ & $<0.26$ & $\ldots$ & $>3280$ & $\ldots$ \\
\hline $\mathrm{J} 1732-3131^{2}$ & $\mathrm{C}$ & $9.39_{-9.39}^{+28.58}$ & $0.37 \pm 0.13$ & Pow & $5260 \pm 1870$ & $\ldots$ \\
\hline J1741-2054² & $\mathrm{C}$ & $15.3_{-3.6}^{+5.1}$ & $6.24_{-1.14}^{+0.34}$ & BB+Pow & $187_{-35}^{+13}$ & $1.68_{-0.34}^{+0.28}$ \\
\hline $\mathrm{J} 1746-3239^{0}$ & $\mathrm{~S}$ & $10^{\mathrm{c}}$ & $<1.74$ & $\ldots$ & $>416$ & . \\
\hline $\mathrm{J} 1747-2958^{2 *}$ & $\mathrm{C}+\mathrm{X}$ & $256_{-6}^{+9}$ & $48.70_{-6.00}^{+21.30}$ & Pow & $43.3_{-6.1}^{+19.2}$ & $84.5_{-4.0}^{+10.2}$ \\
\hline $\mathrm{J} 1801-2451^{2}$ & $\mathrm{C}+\mathrm{X}$ & $374_{-108}^{+120}$ & $9.97 \pm 2.02$ & Pow & $75.3 \pm 45.9$ & $3.27 \pm 1.24$ \\
\hline $\mathrm{J} 1803-2149^{0}$ & $\mathrm{Su}$ & $50^{\mathrm{c}}$ & $<0.46$ & $\ldots$ & $>2030$ & $\ldots$ \\
\hline $\mathrm{J} 1809-2332^{2}$ & $\mathrm{C}+\mathrm{X}$ & $49.2_{-5.5}^{+6.8}$ & $1.32 \pm 0.30$ & $\mathrm{BB}+\mathrm{Pow}$ & $3590 \pm 820$ & $14.4_{-2.2}^{+1.6}$ \\
\hline $\mathrm{J} 1813-1246^{1}$ & $\mathrm{Su}$ & $153_{-50}^{+61}$ & $1.37_{-0.45}^{+0.24}$ & Pow & $1840_{-610}^{+330}$ & $\mathrm{Y}$ \\
\hline $\mathrm{J} 1826-1256^{2}$ & $\mathrm{C}$ & $126_{-46}^{+53}$ & $1.12 \pm 0.25$ & Pow & $3420 \pm 770$ & $1.52 \pm 0.33$ \\
\hline $\mathrm{J} 1833-1034^{2}$ & $\mathrm{X}+\mathrm{C}$ & $210 \pm 1$ & $66.30 \pm 1.50$ & Pow & $8.89 \pm 1.15$ & $721 \pm 5$ \\
\hline $\mathrm{J} 1835-1106^{0}$ & $\mathrm{C}$ & $90^{\mathrm{c}}$ & $<0.28$ & $\ldots$ & & $\ldots$ \\
\hline $\mathrm{J} 1836+5925^{2}$ & $\mathrm{X}+\mathrm{C}$ & $0.7_{-0.7}^{+10.6}$ & $0.31_{-0.21}^{+0.04}$ & $\mathrm{BB}+\mathrm{Pow}$ & $19500_{-13400}^{+2300}$ & $\mathrm{~N}$ \\
\hline
\end{tabular}


Table 15

(Continued)

\begin{tabular}{|c|c|c|c|c|c|c|}
\hline $\mathrm{PSR}^{\mathrm{a}}$ & Inst $^{\mathrm{b}}$ & $\begin{array}{c}N_{\mathrm{H}} \\
\left(10^{20} \mathrm{~cm}^{-2}\right)\end{array}$ & $\begin{array}{c}F_{X}^{\mathrm{nt}} \\
\left(10^{-13} \mathrm{erg} \mathrm{cm}^{-2} \mathrm{~s}^{-1}\right)\end{array}$ & $\begin{array}{l}\text { Sp. Type, } \\
\text { Pulsed }^{\text {d }}\end{array}$ & $G_{100} / F_{X}^{\mathrm{nt}}$ & $\begin{array}{c}F_{X}^{\mathrm{pwn}} \\
\left(10^{-13} \mathrm{erg} \mathrm{cm}^{-2} \mathrm{~s}^{-1}\right)\end{array}$ \\
\hline $\mathrm{J} 1838-0537^{1 *}$ & $\mathrm{Su}$ & $100^{c}$ & $0.88 \pm 0.07$ & Pow & $2130 \pm 230$ & $\ldots$ \\
\hline $\mathrm{J} 1846+0919^{0}$ & $\mathrm{~S}$ & $20^{\mathrm{c}}$ & $<2.92$ & $\ldots$ & $>83.3$ & $\ldots$ \\
\hline $\mathrm{J} 1907+0602^{2}$ & $\mathrm{X}+\mathrm{C}+\mathrm{Su}$ & $41.1_{-3.0}^{+3.5}$ & $0.58 \pm 0.14$ & Pow & $4410 \pm 1050$ & $\mathrm{~N}$ \\
\hline $\mathrm{J} 1952+3252^{2}$ & $\mathrm{X}+\mathrm{C}$ & $33.3 \pm 0.9$ & $40.70 \pm 1.50$ & $\mathrm{BB}+\mathrm{Pow}$ & $33.9 \pm 1.8$ & $77.7 \pm 1.5$ \\
\hline $\mathrm{J} 1954+2836^{0}$ & $\mathrm{Su}$ & $50^{\mathrm{c}}$ & $<0.75$ & $\ldots$ & $>1370$ & $\ldots$ \\
\hline $\mathrm{J} 1957+5033^{0}$ & $\mathrm{Su}$ & $10^{\mathrm{c}}$ & $<0.33$ & $\ldots$ & $>810$ & $\ldots$ \\
\hline $\mathrm{J} 1958+2846^{2}$ & $\mathrm{C}+\mathrm{Su}$ & $122_{-54}^{+71}$ & $1.37 \pm 0.66$ & Pow & $667 \pm 325$ & $\mathrm{~N}$ \\
\hline $\mathrm{J} 2021+3651^{2}$ & $\mathrm{C}+\mathrm{X}$ & $63.8_{-0.39}^{+0.50}$ & $2.15_{-0.49}^{+0.24}$ & $\mathrm{BB}+\mathrm{Pow}$ & $2300_{-530}^{+260}$ & $10.4 \pm 0.6$ \\
\hline $\mathrm{J} 2021+4026^{2}$ & $\mathrm{C}$ & $65.2_{-37.3}^{+30.5}$ & $0.15 \pm 0.01$ & $\mathrm{BB}+\mathrm{Pow}$ & $64600 \pm 4000$ & $\mathrm{~N}$ \\
\hline $\mathrm{J} 2028+3332^{0}$ & S & $10^{\mathrm{c}}$ & $<1.57$ & $\ldots$ & $>370$ & $\ldots$ \\
\hline $\mathrm{J} 2030+3641^{0}$ & S & $80^{c}$ & $<4.52$ & $\ldots$ & $>69.5$ & $\ldots$ \\
\hline $\mathrm{J} 2030+4415^{0}$ & S & $40^{\mathrm{c}}$ & $<2.53$ & $\ldots$ & $>228$ & $\ldots$ \\
\hline $\mathrm{J} 2032+4127^{2}$ & $\mathrm{C}+\mathrm{X}$ & $47.8_{-14.9}^{+13.1}$ & $0.27_{-0.16}^{+0.14}$ & Pow & $5110_{-2950}^{+2630}$ & $\ldots$ \\
\hline $\mathrm{J} 2043+2740^{2}$ & $\mathrm{X}$ & $<3.62$ & $0.22_{-0.11}^{+0.03}$ & Pow & $453_{-255}^{+117}$ & $\cdots$ \\
\hline $\mathrm{J} 2055+2539^{2}$ & $\mathrm{X}$ & $15.1_{-11.7}^{+14.2}$ & $0.43_{-0.28}^{+0.12}$ & Pow & $1240_{-800}^{+350}$ & $2.61 \pm 0.84$ \\
\hline $\mathrm{J} 2111+4606^{0}$ & $\mathrm{~S}$ & $30^{c}$ & $<2.25$ & $\ldots$ & $>196$ & $\ldots$ \\
\hline $\mathrm{J} 2139+4716^{0}$ & $\mathrm{~S}$ & $10^{\mathrm{c}}$ & $<3.20$ & $\ldots$ & $>73.1$ & $\ldots$ \\
\hline $\mathrm{J} 2229+6114^{2}$ & $\mathrm{C}+\mathrm{X}$ & $30_{-4}^{+9}$ & $51.30_{-5.80}^{+9.30}$ & Pow, P & $49.4_{-5.7}^{+9.0}$ & $11.4_{-1.0}^{+0.8}$ \\
\hline $\mathrm{J} 2238+5903^{0}$ & $\mathrm{~S}$ & $70^{c}$ & $<4.49$ & $\ldots$ & $>143$ & $\ldots$ \\
\hline $\mathrm{J} 2240+5832^{0}$ & S & $70^{c}$ & $<4.60$ & $\ldots$ & $>23.5$ & $\ldots$ \\
\hline
\end{tabular}

Notes. X-ray characteristics of young LAT pulsars. The listed fluxes are unabsorbed and non-thermal in the $0.3-10 \mathrm{keV}$ energy band. The model used is an absorbed power law, plus a blackbody (BB) when statistically necessary. The exceptions are PSRs J0633+1746 and J0659+1414 (double BB plus power law). The errors are at the $90 \%$ confidence level. For type 1 and $1 *$ pulsars we assumed that the PWN and pulsar thermal contributions are $30 \%$ of the entire source flux. X-ray nebulae have been detected (or excluded) through brilliance profile analyses; when spectral analysis was possible the flux is in the last column, otherwise the confirmed (or not) presence of a PWN is noted by "Y" ("N").

a Superscripts: 0: no X-ray detection, or no non-thermal component to the X-ray spectrum; 1: X-ray spectrum is poorly constrained; 2: Non-thermal X-ray source (see Section 9.1). An asterisk means an ad hoc analysis was necessary. The $1 *$ indicates a Suzaku detection positionally consistent with a Fermi source, see Marelli et al. (2011).

${ }^{\mathrm{b}} \mathrm{C}=$ Chandra/ACIS, $\mathrm{X}=$ XMM-Newton/PN+MOS, S = Swift/XRT, Su = Suzaku/XIS. For L, the results were taken from Kargaltsev \& Pavlov (2008) for the Crab, from De Luca et al. (2005) for Geminga and J0659+1414, and from Mori et al. (2004) for Vela.

${ }^{c}$ The column density $N_{\mathrm{H}}$ was set to the Galactic value for the pulsar direction obtained with Webtools (http://heasarc.gsfc.nasa.gov/docs/tools.html), scaled for the distance.

d "P" indicates observation of pulsed X-rays.

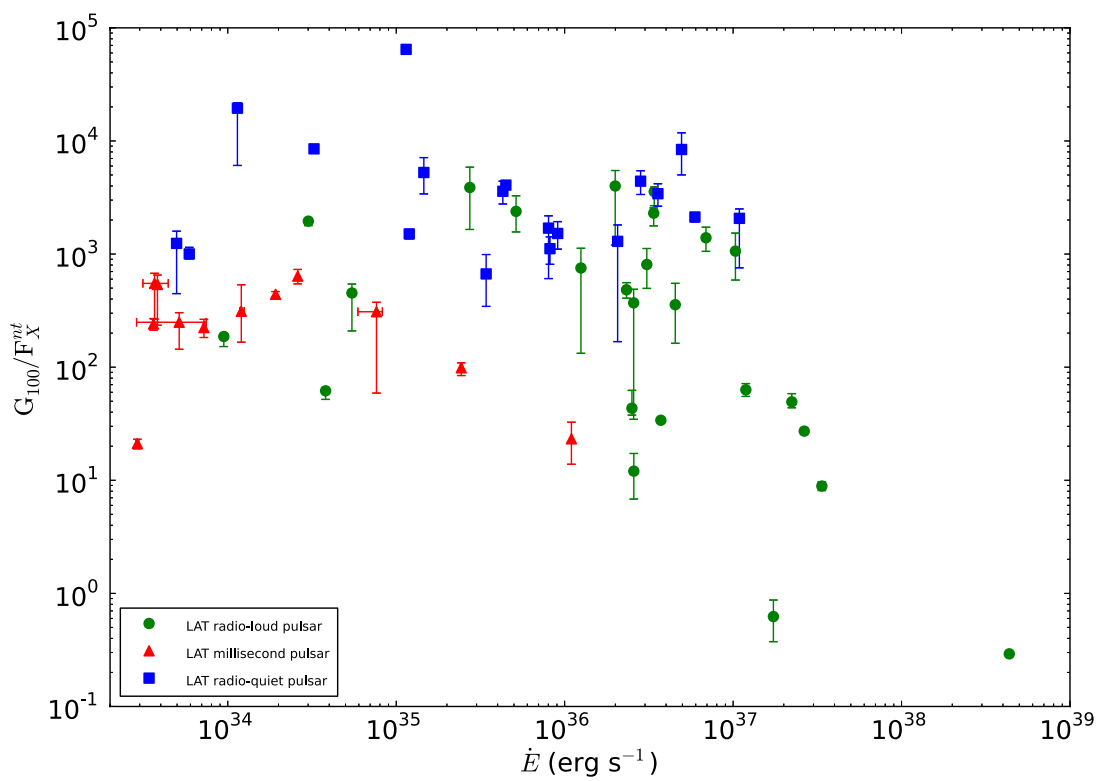

Figure 18. Ratio of $G_{100}$ to the unabsorbed non-thermal X-ray flux vs. $\dot{E}$ for pulsars with good X-ray spectral measurements ("type 2 "). All $G_{100}$ values are included, even when flagged with a $\dagger$ in Tables 9 and 10 . Correlation with $\dot{E}$, if any, is weak. The markers are the same as in Figure 1 . The young radio-loud pulsars have $\left\langle\log \left(G_{100} / F_{X}\right)\right\rangle=2.4 \pm 1.1$, the radio-quiet population has $\left\langle\log \left(G_{100} / F_{X}\right)\right\rangle=3.5 \pm 0.5$, while the MSPs have $\left\langle\log \left(G_{100} / F_{X}\right)\right\rangle=2.3 \pm 0.5$.

(A color version of this figure is available in the online journal.) 
Table 16

X-Ray Spectral Parameters of LAT-detected MSPs

\begin{tabular}{|c|c|c|c|c|c|c|}
\hline $\mathrm{PSR}^{\mathrm{a}}$ & Inst $^{\mathrm{b}}$ & $\begin{array}{c}N_{\mathrm{H}} \\
\left(10^{20} \mathrm{~cm}^{-2}\right)\end{array}$ & $\begin{array}{c}F_{X}^{\mathrm{nt}} \\
\left(10^{-13} \mathrm{erg} \mathrm{cm}^{-2} \mathrm{~s}^{-1}\right)\end{array}$ & $\begin{array}{l}\text { Sp.Type, } \\
\text { Pulsed }^{\text {d }}\end{array}$ & $G_{100} / F_{X}^{\mathrm{nt}}$ & $\begin{array}{c}F_{X}^{\mathrm{pwn}} \\
\left(10^{-13} \mathrm{erg} \mathrm{cm}^{-2} \mathrm{~s}^{-1}\right)\end{array}$ \\
\hline $\mathrm{J} 0023+0923^{1}$ & $\mathrm{C}$ & $5^{\mathrm{c}}$ & $0.21_{-0.17}^{+0.20}$ & Pow & $381_{-321}^{+374}$ & $\ldots$ \\
\hline $\mathrm{J} 0030+0451^{2}$ & $\mathrm{X}$ & $6.4_{-2.4}^{+3.4}$ & $2.55 \pm 0.29$ & $\mathrm{BB}+$ Pow, $\mathrm{P}$ & $241 \pm 29$ & $\mathrm{~N}$ \\
\hline $\mathrm{J} 0034-0534^{0}$ & $\mathrm{X}$ & $<56.3$ & $<0.06$ & BB & $>2800$ & $\mathrm{~N}$ \\
\hline $\mathrm{J} 0101-6422^{0}$ & $\mathrm{~S}$ & $1^{\mathrm{c}}$ & $<2.31$ & $\ldots$ & $>45.3$ & $\ldots$ \\
\hline $\mathrm{J} 0102+4839^{0}$ & $\mathrm{Su}$ & $5^{\mathrm{c}}$ & $<0.17$ & $\ldots$ & $>777$ & $\cdots$ \\
\hline $\mathrm{J} 0218+4232^{2}$ & $\mathrm{X}$ & $\begin{array}{l}2.70_{-2.70}^{+3.76} \\
w^{2}\end{array}$ & $4.62_{-0.63}^{+0.43}$ & Pow, P & $98.7_{-15.5}^{+12.0}$ & $\mathrm{~N}$ \\
\hline $\mathrm{J} 0340+4130^{0}$ & $\mathrm{X}$ & $5^{\mathrm{c}}$ & $<0.20$ & $\ldots$ & $>1020$ & $\ldots$ \\
\hline $\mathrm{J} 0437-4715^{2}$ & $\mathrm{X}+\mathrm{C}$ & $1.58_{-1.09}^{+0.93}$ & $7.91_{-0.60}^{+0.50}$ & BB+Pow, $\mathrm{P}$ & $21.1_{-2.6}^{+2.5}$ & $\mathrm{~N}$ \\
\hline $\mathrm{J} 0610-2100^{0}$ & $\mathrm{~S}$ & $8^{\mathrm{c}}$ & $<1.15$ & $\ldots$ & $>57.1$ & $\ldots$ \\
\hline $\mathrm{J} 0613-0200^{2 *}$ & $\mathrm{X}$ & $<3.30$ & $0.96_{-0.44}^{+0.69}$ & Pow & $312_{-147}^{+225}$ & $\mathrm{~N}$ \\
\hline J0614-3329 & $\mathrm{X}+\mathrm{Su}$ & $6.44_{-2.01}^{+6.32}$ & $1.41_{-0.58}^{+0.48}$ & Pow & $776_{-320}^{+266}$ & $\mathrm{Y}$ \\
\hline $\mathrm{J} 0751+1807^{2}$ & X & $8.74_{-8.74}^{+2.10}$ & $0.59 \pm 0.09$ & $\mathrm{BB}+\mathrm{Pow}$ & $224 \pm 47$ & $\mathrm{~N}$ \\
\hline $\mathrm{J} 1024-0719^{0}$ & $\mathrm{X}$ & $<3.58$ & $<0.11$ & BB & $>286$ & $\ldots$ \\
\hline $\mathrm{J} 1124-3653^{1}$ & $\mathrm{~S}$ & $5^{c}$ & $0.45 \pm 0.25$ & Pow & $269 \pm 156$ & $\ldots$ \\
\hline $\mathrm{J} 1125-5825^{0}$ & $\ldots$ & $\ldots$ & $\ldots$ & $\ldots$ & $\ldots$ & $\ldots$ \\
\hline $\mathrm{J} 1231-1411^{2}$ & $X$ & $11.3 \pm 5.1$ & $4.12_{-1.74}^{+0.88}$ & $\mathrm{BB}+\mathrm{Pow}$ & $250_{-106}^{+54}$ & $\mathrm{~N}$ \\
\hline $\mathrm{J} 1446-4701^{0}$ & $\mathrm{~S}$ & $10^{\mathrm{c}}$ & $<1.50$ & $\ldots$ & $>49.5$ & $\ldots$ \\
\hline $\mathrm{J} 1514-4946^{0}$ & $\mathrm{C}$ & $50^{\mathrm{c}}$ & $<0.16$ & $\ldots$ & $>2760$ & $\ldots$ \\
\hline $\mathrm{J} 1600-3053^{0}$ & $\mathrm{X}$ & $10^{\mathrm{c}}$ & $<0.07$ & $\mathrm{BB}$ & $>2500$ & $\ldots$ \\
\hline $\mathrm{J} 1614-2230^{0}$ & $\mathrm{C}+\mathrm{X}$ & $2.9_{-2.9}^{+4.3}$ & $<0.29$ & $\mathrm{BB}$ & $>852$ & $\mathrm{~N}$ \\
\hline $\mathrm{J} 1658-5324^{1}$ & $\mathrm{C}$ & $<14.9$ & $1.24_{-0.80}^{+0.39}$ & Pow & $233_{-153}^{+78}$ & $\ldots$ \\
\hline $\mathrm{J} 1713+0747^{0}$ & $\mathrm{~S}$ & $5^{\mathrm{c}}$ & $<1.80$ & $\ldots$ & $>56.5$ & $\ldots$ \\
\hline $\mathrm{J} 1741+1351^{0}$ & $S$ & $5^{\mathrm{c}}$ & $<2.30$ & Pow & $>10.5$ & $\ldots$ \\
\hline $\mathrm{J} 1744-1134^{0}$ & $\mathrm{C}$ & $9.40_{-9.40}^{+11.50}$ & $<0.26$ & $\mathrm{BB}$ & $>1270$ & $\mathrm{~N}$ \\
\hline $\mathrm{J} 1747-4036^{0}$ & $\mathrm{~S}$ & $20^{\mathrm{c}}$ & $<1.80$ & $\ldots$ & $>55.1$ & $\ldots$ \\
\hline $\mathrm{J} 1810+1744^{1}$ & $\mathrm{C}$ & $6_{-6}^{+20}$ & $0.18 \pm 0.07$ & Pow & $1290 \pm 520$ & $\ldots$ \\
\hline $\mathrm{J} 1823-3021 \mathrm{~A}^{0}$ & $\mathrm{X}+\mathrm{C}$ & $\ldots$ & $\ldots$ & $\ldots$ & $\ldots$ & $\ldots$ \\
\hline $\mathrm{J} 1858-2216^{0}$ & $\mathrm{~S}$ & $10^{\mathrm{c}}$ & $<1.95$ & $\ldots$ & $>37.1$ & $\ldots$ \\
\hline $\mathrm{J} 1902-5105^{0}$ & $\mathrm{Su}$ & $3^{\mathrm{c}}$ & $<0.56$ & $\ldots$ & $>382$ & $\cdots$ \\
\hline $\mathrm{J} 1939+2134^{2}$ & $\mathrm{C}$ & $109_{-44}^{+63}$ & $3.95 \pm 0.71$ & Pow, P & $23.2 \pm 13.3$ & $\mathrm{~N}$ \\
\hline $\mathrm{J} 1959+2048^{2}$ & $\mathrm{X}+\mathrm{C}$ & $3.72_{-3.72}^{+3.79}$ & $0.55_{-0.44}^{+0.10}$ & $\mathrm{BB}+\mathrm{Pow}$ & $309_{-253}^{+76}$ & $0.168_{-0.071}^{+0.061}$ \\
\hline $\mathrm{J} 2017+0603^{1}$ & $\mathrm{C}$ & $10^{\mathrm{c}}$ & $0.11 \pm 0.02$ & Pow & $3030 \pm 620$ & $\ldots$ \\
\hline $\mathrm{J} 2043+1711^{0}$ & $\mathrm{~S}$ & $6^{c}$ & $<0.98$ & $\ldots$ & $>276$ & $\ldots$ \\
\hline $\mathrm{J} 2047+1053^{0}$ & $\mathrm{~S}$ & $7^{\mathrm{c}}$ & $<1.90$ & $\ldots$ & $>32.5$ & $\ldots$ \\
\hline $\mathrm{J} 2051-0827^{0}$ & $\mathrm{C}+\mathrm{X}$ & $<17.1$ & $<0.04$ & $\mathrm{BB}$ & $>1530$ & $\ldots$ \\
\hline $\mathrm{J} 2124-3358^{2}$ & $\mathrm{X}$ & $2.76_{-2.76}^{+4.87}$ & $0.67_{-0.34}^{+0.15}$ & $\mathrm{BB}+$ Pow, $\mathrm{P}$ & $550_{-286}^{+129}$ & $0.140_{-0.069}^{+0.094}$ \\
\hline $\mathrm{J} 2214+3000^{2}$ & $\mathrm{C}$ & $<21.3$ & $0.74 \pm 0.03$ & Pow & $441 \pm 34$ & $\ldots$ \\
\hline $\mathrm{J} 2215+5135^{1}$ & $\mathrm{C}$ & $10^{\mathrm{c}}$ & $0.77 \pm 0.35$ & Pow & $153 \pm 75$ & $\ldots$ \\
\hline $\mathrm{J} 2241-5236^{2}$ & $\mathrm{C}$ & $<24.8$ & $0.52 \pm 0.07$ & Pow & $638 \pm 99$ & $\ldots$ \\
\hline $\mathrm{J} 2302+4442^{2}$ & $\mathrm{X}$ & $13.0_{-5.2}^{+9.1}$ & $0.68_{-0.38}^{+0.14}$ & Pow & $539_{-305}^{+117}$ & $\mathrm{~N}$ \\
\hline
\end{tabular}

Notes. X-ray characteristics of LAT MSPs. The listed fluxes are unabsorbed and non-thermal in the $0.3-10 \mathrm{keV}$ energy band. The model used is an absorbed power law, plus a blackbody (BB) component when statistically necessary. The exceptions is PSR J0437-4715 (double BB plus power law). The errors are at the $90 \%$ confidence level. For type 1 and $1 *$ pulsars we assumed that the PWN and PSR thermal contributions are $30 \%$ of the entire source flux. X-ray nebulae have been detected (or excluded) through brilliance profile analyses; when spectral analysis was possible the flux is in the last column of the table, otherwise the confirmed (or not) presence of a PWN is noted by "Y" ("N").

a Superscripts: 0: no X-ray detection, or no non-thermal component to the X-ray spectrum; 1: X-ray spectrum is poorly constrained; 2: Non-thermal X-ray source (see Section 9.1). An asterisk means an ad hoc analysis was necessary.

${ }^{\mathrm{b}} \mathrm{C}=$ Chandra/ACIS, $\mathrm{X}=$ XMM-Newton $/ \mathrm{PN}+\mathrm{MOS}, \mathrm{S}=$ Swift $/ \mathrm{XRT}, \mathrm{Su}=$ Suzaku/XIS.

${ }^{c}$ The column density $N_{\mathrm{H}}$ was set to the Galactic value for the pulsar direction obtained with Webtools, scaled for the distance.

$\mathrm{d}$ " $\mathrm{P}$ " indicates observation of pulsed X-rays.

\subsection{Optical Properties}

Only seven of the Fermi pulsars are firmly identified at ultraviolet (UV), optical and/or infrared (IR) wavelengths. Six are solitary young-to-middle-aged pulsars (Crab, Vela, PSR B1509-58, PSR B0656+14, Geminga, PSR B1055-52), all detected in the optical and some also in the IR and UV. One is an MSP in a binary system, only detected in the near-UV
(PSR J0437-4715). All were identified prior to the launch of Fermi, mostly in the 1990s (Mignani 2011). In the last decade possible counterparts were found for PSR B1951+32 (Butler et al. 2002) and the solitary MSP PSR J1024-0719 (Sutaria et al. 2003), prior to their detection as gamma-ray pulsars. Furthermore, PSR J1124-5916 has been associated with a bright, optical PWN, although no point source has been identified as a potential counterpart (Zharikov et al. 
Table 17

Optical Observations of LAT Pulsars

\begin{tabular}{|c|c|c|c|c|c|c|}
\hline PSR & Magnitude & Filter & Extinction & $\begin{array}{c}\text { Corrected Flux } \\
\left(10^{-15} \mathrm{erg} \mathrm{cm}^{-2} \mathrm{~s}^{-1}\right)\end{array}$ & Type $^{\mathrm{a}}$ & References \\
\hline $\mathrm{J} 0007+7303$ & $>27.6$ & $R$ & $0.92_{-0.42}^{+0.49}$ & $<0.076$ & $\mathrm{U}$ & Mignani et al. (2013b) \\
\hline $\mathrm{J} 0023+0923$ & 25 & $g$ & 0.37 & $<0.86$ & $\mathrm{BC}$ & (Breton et al. 2013) \\
\hline $\mathrm{J} 0030+0451$ & $>27.0$ & $V$ & $0.36_{-0.13}^{+0.19}$ & $<0.08$ & $\mathrm{U}$ & Koptsevich et al. (2003) \\
\hline J0034-0534 & 24.80 & $I$ & 3.13 & $<0.66$ & $\mathrm{BC}$ & Lundgren et al. (1996) \\
\hline J0205+6449 & $>25.50$ & $R$ & $2.50_{-0.06}^{+0.07}$ & $<1.10$ & $\mathrm{U}$ & S. Collins et al. (in preparation) \\
\hline $\mathrm{J} 0218+4232$ & 24.20 & $V$ & $0.15_{-0.15}^{+0.21}$ & $<0.75$ & $\mathrm{BC}$ & Bassa et al. (2003) \\
\hline $\mathrm{J} 0248+6021$ & $>20.9$ & $U$ & 4.44 & $<3400$ & $\mathrm{U}$ & Theureau et al. (2011) \\
\hline J0357+3205 & $>27.3$ & $V$ & $0.44 \pm 0.22$ & $<0.07$ & $\mathrm{U}$ & De Luca et al. (2011) \\
\hline J0437-4715 & 24.80 & $V$ & 0.01 & $0.38 \pm 0.001$ & $\mathrm{BP}$ & Kargaltsev et al. (2004) \\
\hline J0534+2200 & 16.50 & $V$ & $1.92 \pm 0.01$ & $4600 \pm 47$ & $\mathrm{P}^{*}$ & Cocke et al. (1969) \\
\hline J0610-2100 & 26.70 & $V$ & 0.44 & $<0.098$ & $\mathrm{BU}$ & Pallanca et al. (2012) \\
\hline J0613-0200 & $>26.0$ & $B$ & 0.18 & $<0.28$ & BU & S. Collins et al. (in preparation) \\
\hline J0631+1036 & $>24.2$ & $V$ & 1.11 & $<1.90$ & $\mathrm{U}$ & S. Collins et al. (in preparation) \\
\hline $\mathrm{J} 0633+0632$ & $>27.4$ & $R$ & $0.34_{-0.34}^{+1.22}$ & $<0.10$ & $\mathrm{U}$ & S. Collins et al. (in preparation) \\
\hline J0633+1746 & 25.50 & $V$ & 0.06 & 0.21 & $\mathrm{P}^{*}$ & Bignami et al. (1993) \\
\hline J0659+1414 & 25.00 & $V$ & $0.24 \pm 0.01$ & $0.39 \pm 0.004$ & $\mathrm{P}^{*}$ & Caraveo et al. (1994) \\
\hline J0742-2822 & $>26.0$ & $V$ & 1.11 & $<0.35$ & $\mathrm{U}$ & $\ldots$ \\
\hline $\mathrm{J} 0751+1807$ & 25.08 & $R$ & $0.49_{-0.49}^{+0.12}$ & $<0.41$ & $\mathrm{BC}$ & Bassa et al. (2006) \\
\hline J0835-4510 & 23.60 & $V$ & $0.12 \pm 0.03$ & $1.3 \pm 0.03$ & $\mathrm{P}^{*}$ & Lasker (1976) \\
\hline J1024-0719 & 24.90 & $V$ & 0.20 & 0.41 & $\mathrm{P}+$ & Sutaria et al. (2003) \\
\hline J1028-5819 & $>25.4$ & $B$ & 2.78 & $<12$ & $\mathrm{U}$ & Mignani et al. (2012b) \\
\hline J1048-5832 & $>27.6$ & $V$ & $2.56 \pm 0.13$ & $<0.34$ & $\mathrm{U}$ & Mignani et al. (2011) \\
\hline J1057-5226 & 25.43 & $V$ & $0.15 \pm 0.01$ & $0.24 \pm 0.003$ & $\mathrm{P}$ & Mignani et al. (2010b) \\
\hline J1119-6127 & $>24.0$ & $J$ & $10.3 \pm 2.2$ & $<2.90$ & $\mathrm{U}$ & Mignani et al. (2007) \\
\hline J1124-5916 & 24.93 & $V$ & $1.67_{-0.27}^{+0.16}$ & 1.5 & $\mathrm{~N}$ & Zharikov et al. (2008) \\
\hline J1231-1411 & $>26.3$ & $V$ & $0.63 \pm 0.28$ & $<0.23$ & $\mathrm{BU}$ & S. Collins et al. (in preparation) \\
\hline J1357-6429 & 24.60 & $I$ & $1.05_{-0.25}^{+0.27}$ & 0.3 & $\mathrm{P}+$ & Mignani et al. (2011) \\
\hline J1413-6205 & $>23.0$ & $R$ & 2.22 & $<9.1$ & $\mathrm{U}$ & $\ldots$ \\
\hline J1418-6058 & $>23.0$ & $R$ & $12.5_{-2.5}^{+2.9}$ & $<80000$ & $\mathrm{U}$ & $\ldots$ \\
\hline J1513-5908 & 26.00 & $R$ & $5.10 \pm 0.01$ & $4.2 \pm 0.032$ & $\mathrm{P}$ & Wagner \& Seifert (2000) \\
\hline J1614-2230 & 24.30 & $R$ & $0.16_{-0.16}^{+0.24}$ & $<0.67$ & $\mathrm{BC}$ & Bhalerao \& Kulkarni (2011) \\
\hline J1709-4429 & $>27.5$ & $V$ & $2.53_{-0.16}^{+0.24}$ & $<0.40$ & $\mathrm{U}$ & Mignani et al. (1999) \\
\hline $\mathrm{J} 1713+0747$ & 26.00 & $V$ & 0.00 & $<0.12$ & $\mathrm{BC}$ & Lundgren et al. (1996) \\
\hline J1718-3825 & $>24.0$ & $V$ & $2.3_{-0.9}^{+0.8}$ & $<13.3$ & $\mathrm{U}$ & $\ldots$ \\
\hline J1744-1134 & $>26.3$ & $V$ & $0.52_{-0.52}^{+0.64}$ & $<0.27$ & $\mathrm{U}$ & Sutaria et al. (2003) \\
\hline J1747-2958 & $>25.0$ & $R$ & $14.22_{-0.50}^{+0.33}$ & $<7200$ & $\mathrm{U}$ & $\ldots$ \\
\hline J1833-1034 & 15.86 & $K$ & $11.67 \pm 0.06$ & 64 & $\mathrm{~N}$ & Zajczyk et al. (2012) \\
\hline $\mathrm{J} 1810+1744$ & 20.20 & $g$ & 0.43 & $<76$ & $\mathrm{BC}$ & Breton et al. (2013) \\
\hline $\mathrm{J} 1836+5925$ & $>28.5$ & $V$ & $0.04_{-0.04}^{+0.59}$ & $<0.02$ & $\mathrm{U}$ & Halpern et al. (2002) \\
\hline $\mathrm{J} 1952+3252$ & 24.50 & $V$ & $1.85 \pm 0.05$ & 2.70 & $\mathrm{P}+$ & Butler et al. (2002) \\
\hline $\mathrm{J} 1959+2048$ & 20.00 & $V$ & $0.21 \pm 0.21$ & $<38$ & $\mathrm{BC}$ & Kulkarni et al. (1988) \\
\hline $\mathrm{J} 2017+0603$ & $>19.1$ & $V$ & 0.56 & $<120$ & $\mathrm{BU}$ & Cognard et al. (2011) \\
\hline $\mathrm{J} 2021+4026$ & $>25.2$ & $R$ & $3.62_{-2.07}^{+1.69}$ & $<10.20$ & $\mathrm{U}$ & Weisskopf et al. (2011) \\
\hline $\mathrm{J} 2051-0827$ & 22.3 & $R$ & 0.95 & $<7.2$ & $\mathrm{BC}$ & Stappers et al. (1996) \\
\hline $\mathrm{J} 2124-3358$ & $>27.8$ & $V$ & $0.15_{-0.15}^{+0.27}$ & $<0.03$ & $\mathrm{U}$ & Mignani \& Becker (2004) \\
\hline $\mathrm{J} 2215+5135$ & 18.70 & $g$ & 1.15 & $<600$ & $\mathrm{BC}$ & Breton et al. (2013) \\
\hline $\mathrm{J} 2229+6114$ & $>23.0$ & $R$ & $1.67_{-0.22}^{+0.50}$ & $<8.80$ & $\mathrm{U}$ & Halpern et al. (2001) \\
\hline J2256-1024 & 26.80 & $g$ & 0.14 & $<0.13$ & $\mathrm{BC}$ & Breton et al. (2013) \\
\hline $\mathrm{J} 2302+4442$ & $>19.6$ & $V$ & $0.72_{-0.29}^{+0.51}$ & $<145$ & $\mathrm{BU}$ & Cognard et al. (2011) \\
\hline
\end{tabular}

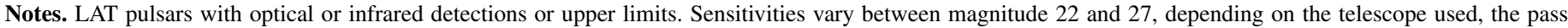

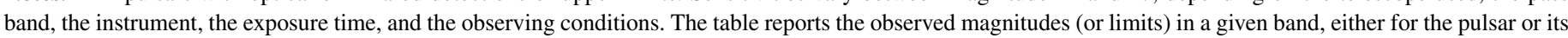

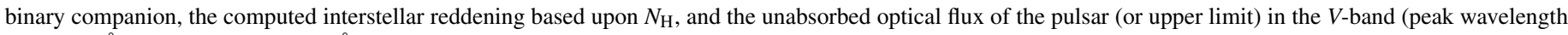
$\lambda=5500 \AA$; bandwidth $\Delta \lambda=890 \AA$ ).

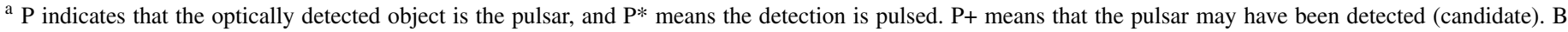

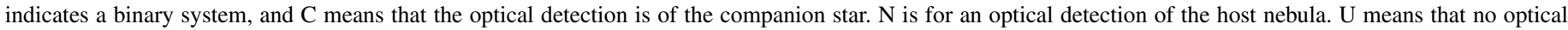

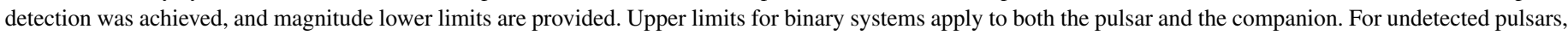
a measured PWN or companion flux is taken as a conservative upper limit on the pulsar flux. 


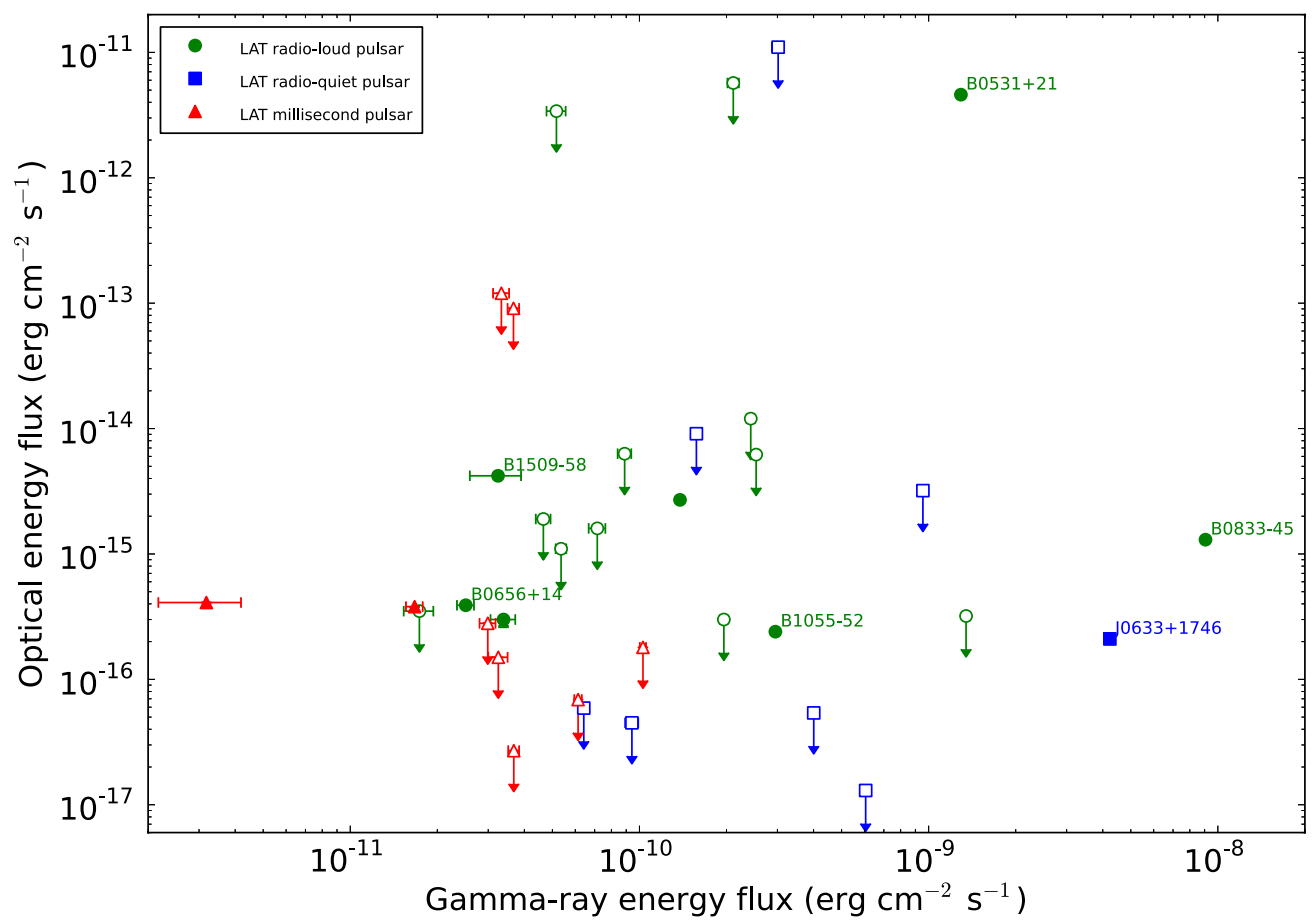

Figure 19. Optical energy fluxes and upper limits when available (see Table 17) vs. the 0.1 to $100 \mathrm{GeV}$ gamma-ray energy fluxes. All $G_{100}$ values are included, even when flagged with a $\nmid$ in Tables 9 and 10. The markers are the same as in Figure 1.

(A color version of this figure is available in the online journal.)

2008). While companion stars have been identified for eight of the binary Fermi pulsars, with four more obtained recently (Breton et al. 2013), our discussion is focused on the optical emission properties of the pulsars only, and not of their binary companions. Table 17 summarizes these results, and includes 49 Fermi pulsars, both solitary and binary.

Since the launch of Fermi there have been no deep systematic optical observations of gamma-ray pulsars. A quick-look survey carried out with 2-4 m class telescopes did not discover any potential counterparts (Collins et al. 2011). Dedicated follow-up observations with either the Hubble Space Telescope or $8 \mathrm{~m}$-class telescopes have been made in a few cases, e.g., for PSR B1055-52 (Mignani et al. 2010b, 2010a), PSR J1357-6429 (Mignani et al. 2011; Danilenko et al. 2012), PSR J1048-5832 (Mignani et al. 2011; Razzano et al. 2013), PSR J1028-5819 (Mignani et al. 2012b), PSR J0205+6449 (Shearer et al. 2013), and PSR J0007+7303 (Mignani et al. 2013b). Apart from PSR B1055-52 for which an optical counterpart had been previously identified, new counterparts were detected only for PSR J1357-6429 and PSR J0205+6449. A bright, near-IR PWN was also found coincident with PSR J1833-1034 (Zajczyk et al. 2012).

We derived optical upper limits for gamma-ray pulsars in two ways: by compiling information from previous publications and by searching public optical archives for unpublished or serendipitous observations of Fermi pulsars. We did not include observations from optical/IR sky surveys, as the limiting fluxes of these surveys are usually too shallow to derive constraining upper limits on the pulsar optical/IR emission. We considered both solitary pulsars and pulsars in binary systems since, in the latter case, the upper limit applies to both the pulsar and its companion. For pulsars associated with PWNe or with a detected binary companion, we assumed the nebula or the companion flux as a very conservative upper limit on the pulsar emission. In all, upper limits exist for 38 Fermi pulsars, though with different sensitivity limits.

Pulsars are located at different distances and are affected by different amounts of interstellar extinction. To investigate their emission properties at optical energies, we first computed the extinction-corrected energy fluxes in the different pass bands. To avoid bias in comparing with X-ray energy flux densities, we used the hydrogen column density $N_{\mathrm{H}}$ derived from the X-ray spectral fits (Marelli et al. 2011) for this calculation. We derived the interstellar reddening $E(B-V)$ from $N_{\mathrm{H}}$, using the relation of Predehl \& Schmitt (1995) with $R_{V} \equiv A_{V} / E(B-V)=3.1$, and computed the extinction in the different pass bands according to the differential extinction coefficients of Fitzpatrick (1999). We note that the uncertainties of the extinction values derived in this way are dominated by the accuracy on the $N_{\mathrm{H}}$ determination from the X-ray spectral fits and the uncertainty on the $N_{\mathrm{H}} / E(B-V)$ ratio that depends on the dust-to-gas ratio along the line of sight and the grain properties. Ideally, to mitigate these uncertainties, one should directly measure the $E(B-V)$ using color-magnitude diagram comparison techniques, as done by Mignani et al. (2013a), for example. However, this requires suitable multiband optical/ IR data over a sufficiently large angular scale for all pulsars listed in Table 17 and photometric analysis for each field, which is beyond the goal of this work. For consistency, we use $V$-band measurements where available. For the other cases, we extrapolated the measured flux or upper limit in the pass band closest to the $V$ band assuming either the measured spectrum for the identified pulsars or, as a first approximation, a power law with spectral index $\alpha_{O}=0$ for the unidentified ones. While the true spectral index of a given pulsar may differ from that we have assumed, we note that in most cases the uncertainty of the 
extinction-corrected flux upper limits due to this assumption is negligible compared to the uncertainty of the interstellar extinction correction.

Figure 19 shows the extinction-corrected optical energy flux plotted against the gamma-ray energy flux for 38 pulsars from Table 17 (binary pulsars where the companion star is detected are excluded). No correlation is apparent but it is clear that the gamma-ray energy flux is much greater than the optical energy flux for all of these pulsars. This is due, in part, to the fact that only seven pulsars are firmly detected in the optical. In addition, the different sensitivities of the observations produce a rather inhomogeneous set of upper limits. If one excludes the Crab and considers only the faintest detected pulsars (J0437-4715, J1057-5226, and Geminga) the optical energy flux appears to be independent of the gamma-flux across about three orders of magnitude. This is surprising as the optical luminosity ( $L_{\text {opt,IR }} \propto \dot{E}^{1.70 \pm 0.03}$; Mignani et al. 2012a) and the gamma-ray luminosity ( $L_{\gamma} \propto \dot{E}^{1 / 2}$; see Figure 9$)$ both scale as a power of the rotational energy loss $\dot{E}$, albeit with different slopes. Because of this mutual dependence, one would expect the optical luminosity, and hence the unabsorbed optical energy flux, to scale with $L_{\gamma}$. However, the luminosity- $\dot{E}$ relation in the optical is computed from a very limited sample of objects and is sensitive to possible outliers. Expanding the sample of gamma-ray pulsars detected in the optical is therefore crucial to establishing possible correlations between the emission in the two energy bands.

\section{DISCUSSION}

This catalog expands on the results of 1PC, with the uniformly analyzed pulsar sample growing from 46 (six months) to 117 (36 months). Nearly half these pulsars were unknown before Fermi, having either been discovered in blind gammaray searches or in LAT-directed radio searches. These pulsars fall nearly equally into three main classes: young radio-loud, young radio-quiet, and millisecond. Compared to $1 \mathrm{PC}$, the larger sample and detailed, uniform analysis of the 117 gamma-ray pulsars in this catalog enables more extensive population studies and evaluations of pulsar models. Although the present work is not an exhaustive review of such work, the following sections outline some of the implications of the catalog. A striking change in the pulsar sample is the dramatic increase in the MSP fraction, $34 \%$ (40/117). This doubles the 1PC fraction and is a testimony to the remarkable success of radio follow-up studies (Ray et al. 2012).

\subsection{Radio and Gamma-Ray Detectability}

In our sample, $53 \%(41 / 77)$ of the young pulsars are radio loud (see Table 1), close to the 55\% (21/38) fraction in 1PC. Because the sensitivity for blind searches is lower than for simple folding (see Section 8.2), the parent population must contain substantially more radio-quiet than radio-loud pulsars (to a given gamma-ray flux limit). This again is quite similar to the inference 1PC. By contrast, all known gamma-ray MSPs are radio loud (MSP J1311-3430 was detected in a blind search of LAT data, but radio pulsations were subsequently detected, Pletsch et al. 2012c; Ray et al. 2013). Although this circumstance might seem to be just a result of the difficulty of blind searches for MSPs, an analysis first done by Romani (2012) showed that the lack of gamma-ray MSPs without detectable radio emission is not an artifact. Among the 250 brightest 2FGL sources, for which the counterpart identifications are nearly complete and

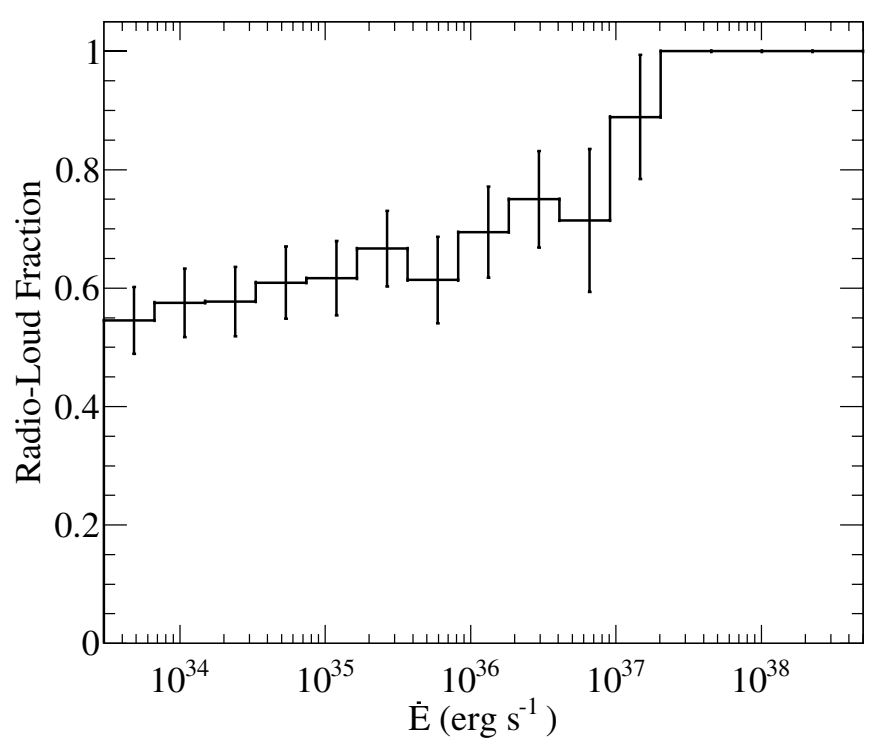

Figure 20. Fraction of young gamma-ray pulsars above a given $\dot{E}$ which are radio-loud, ranging from $\sim 0.5$ for the least energetic to 1.0 for the most energetic.

all sources have sensitive blind searches, only four remain unassociated with objects seen at longer wavelengths. Within this sample, only $41 \%(17 / 41)$ of the young pulsars are radio loud, while all 12 of the MSPs are radio loud. Even if all four of the remaining unassociated sources in this sample are gammaray MSPs with radio beams that do not cross our line of sight (a highly unlikely scenario) the radio-loud MSP fraction can be no smaller than $75 \%$, a much larger fraction than seen for young pulsars.

The detected gamma-ray pulsars are clearly highly energetic, with no young pulsars and only a few MSPs detected below $\dot{E} \approx 3 \times 10^{33} \mathrm{erg} \mathrm{s}^{-1}$ (Figure 1). There are apparent differences in detectability between the pulsar classes, which likely reflect differences in the radio and gamma-ray beaming. Figure 20 shows that the fraction of radio-loud young gamma-ray pulsars increases with $\dot{E}$ (a feature first noted by Ravi et al. 2010). For $\dot{E}>1 \times 10^{37} \mathrm{erg} \mathrm{s}^{-1}$, only one of the nine pulsars is radio-quiet. This may be an effect of the size of the magnetosphere rather than the spindown power, supported by the observation that there is only one radio-quiet pulsar with $P<70 \mathrm{~ms}$. Thus, for gamma-ray pulsars with light-cylinder radius $R_{\mathrm{LC}}<200 R_{\mathrm{NS}}$, one nearly always detects the radio beam.

Watters \& Romani (2011) showed that these trends can indeed be explained by beaming evolution. They found that if radio beams move to high altitudes for young, short period pulsars, as argued by Karastergiou \& Johnston (2007), then nearly all such gamma-ray pulsars are also radio detectable. Also, the increased fraction of low- $\dot{E}$ radio-quiet pulsars was shown to be a natural consequence of outer-magnetosphere models with emission dominated by regions above the null charge surface, especially if the pulsars evolve toward alignment (decreasing $\alpha$ ) on Myr timescales (Young et al. 2010). This is because the radio beam, increasingly aligned to the spin pole, is seldom visible when one views the gamma-ray beam, which is concentrated to the spin equator. Pierbattista et al. (2012) also discuss the lack of high- $\dot{E}$ radio-quiet pulsars and the increase in the radio-quiet fraction at low- $\dot{E}$, plausibly addressed by an evolution toward spin alignment. However, these authors note that such alignment on Myr timescales cannot address the discrepancy seen at high$\dot{E}$ because of the young age of those objects. Also, while Watters 
\& Romani (2011) find that the outer gap (OG; see Cheng et al. 1986; Romani \& Yadigaroglu 1995) model is preferred over the lower-altitude two-pole caustic (TPC; see Dyks \& Rudak 2003) model with very large statistical significance, Pierbattista et al. (2012) argue that a slot gap (SG; see Muslimov \& Harding 2004) model extending to the light cylinder can provide acceptable numbers of pulsar detections and radio-loud/radio-quiet ratios. They also stress that, unless the radio beam is broader than currently modeled, both the SG and OG geometries fail to reproduce the $100 \%$ radio-loud fraction found at high $\dot{E}$. Further work is needed to determine whether any of these models can reproduce the detailed ratios and their evolution with spin period and spindown power.

Compared with the young, radio-loud pulsars, we see in Figures 2 and 4 that MSPs are often detected at smaller distances because of their lower luminosities and at larger Galactic latitude $|b|$ because of the much greater age of this population. The radioquiet young pulsars are intermediate in this respect, reflecting the increased tendency noted above for gamma-only detection as the pulsars spin down. In general MSPs lie within $2 \mathrm{kpc}$, although the detection of the MSP J1823-3021A in the globular cluster NGC 6624 (Freire et al. 2011) illustrates that very energetic (young) MSPs in low background regions can be detectable by the LAT across much of the Galaxy.

In Figure 15, it is apparent that a large fraction of the MSPs above the LAT sensitivity threshold are detected as gammaray pulsars. Thus, the absence of gamma-ray only MSPs and the paucity of above-threshold radio MSPs undetected by the LAT suggests that MSP radio and gamma-ray beams cover a comparably-sized, and nearly-coincident, fraction of the sky.

\subsection{Light Curve Trends}

Additional clues to the pulsar beaming and detectability can be extracted from patterns in the radio and gamma-ray light curves. For young pulsars, the trends visible in 1PC are strengthened and extended in this catalog. Most (58/77) of these pulsars show two strong, caustic peaks significantly separated, often with significant bridge emission. The most prominent light curve trend is the anti-correlation (Figure 5) between $\Delta$ and $\delta$, shown by Romani \& Yadigaroglu (1995) to be a general property of outer-magnetosphere models with caustic pulses. However, the sample in this catalog makes it clear that the trend is not universally followed. Certainly, MSPs show less correlation, and a significant number of young pulsars have only one strong gamma-ray peak. Watters \& Romani (2011) argued that many single-peak, young pulsars fall in the $\delta \approx 0.3-0.6$ range, which can be attributed to either missing the first peak (P1) or a blended combination of P1 and the second peak (P2). However several objects, especially MSPs, depart strongly from this pattern. The LAT MSPs show, on average, larger radio lags than young pulsars, as expected since MSPs have smaller magnetospheres and hence stronger aberration of the radio pulses at typical radio emission heights. The distribution in $\Delta$ for MSPs, shown in the side histogram of Figure 5, also shows a larger fraction with $\Delta>0.5$ than for young pulsars.

One feature noted in 1PC that persists in the current sample is that for pulsars with two strong caustic peaks, the $\mathrm{P} 2 / \mathrm{P} 1$ ratio increases with energy. This often helps us identify the harder P2 component. However, when $\Delta \sim 0.5$ the spectral evolution of the peak ratio is often weak, making peak assignment more difficult (e.g., PSR J0908-4913). For a few single-peak pulsars with sharp trailing edges, we can see the peak strength grow with photon energy - for these objects we suspect that the observed

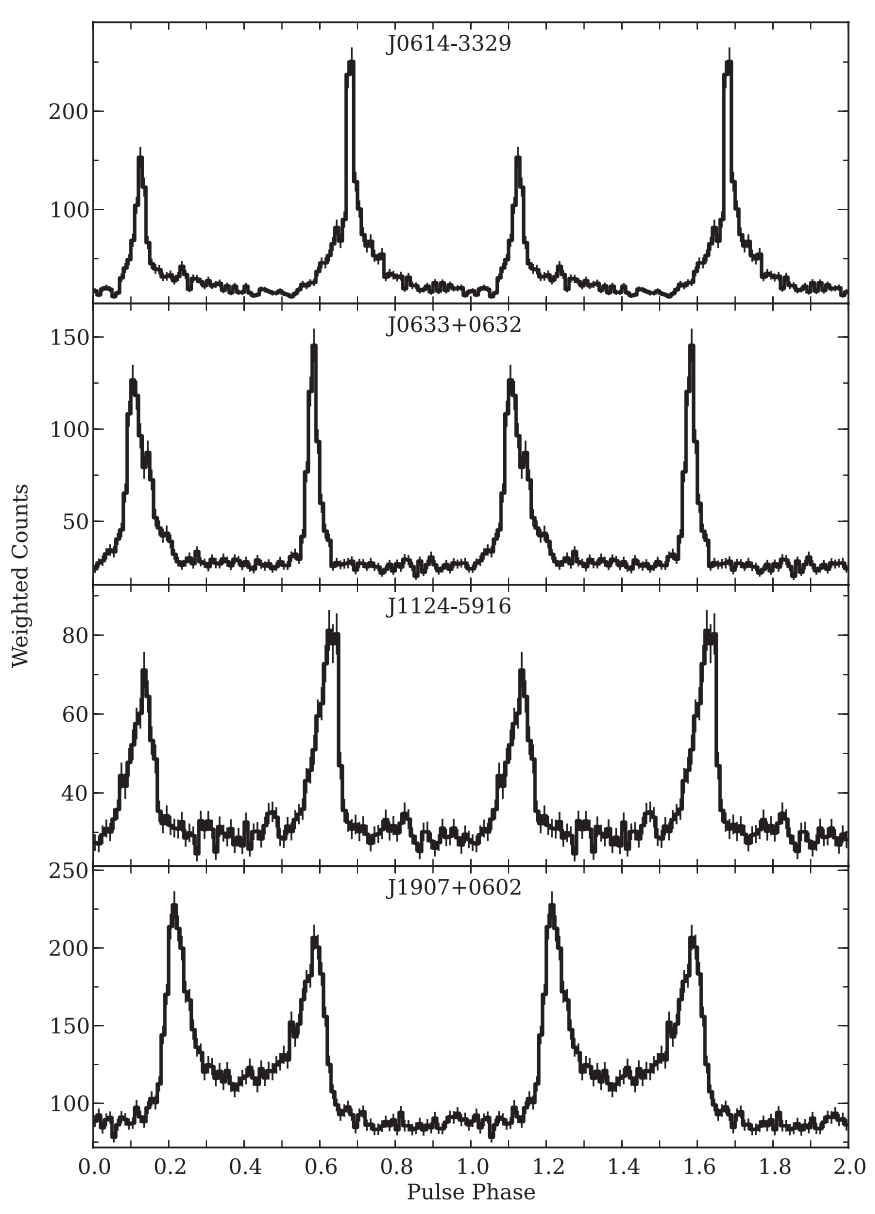

Figure 21. Examples of four types of two-peak gamma-ray pulse profiles: (a) PSR J0614-3329: sharpest peak edge between the two peaks, (b) PSR J0633+0632: sharp leading edge for both peaks, (c) PSR J1124-5916: sharp trailing edge for both peaks, and (d) PSR J1907+0602: "outside" peak edges are sharpest.

peak is a P2 component and P1 may be detectable only below the LAT energy band.

The uniformity of the light curve fitting in this catalog (Section 5.2) should greatly facilitate use of the $\delta-\Delta$ distribution to test magnetospheric models. For example, in such a treatment of the 1PC sample, Watters \& Romani (2011) found that the data gave a strong statistical preference for an OG geometry to that of the TPC model. However, accurate $\delta$ values need the true phase of the magnetic axis, likely requiring careful modeling of the radio light curve and polarization. In addition, magnetospheric currents (see Kalapotharakos et al. 2012a) can provide systematic $\delta$ shifts with respect to the vacuum approximation. Even when radio emission is not detected the $\Delta$ distribution can provide a statistical test of the beaming model. For example, the preponderance of $\Delta \approx 0.5$ is very natural in $\mathrm{SG}$ or TPC models. Figure 6 does not show any strong correlation of $\Delta$ with $\dot{E}$, although some increase in the incidence of smaller $\Delta$ is expected in OG models for lower $\dot{E}$.

The majority of pulsars have two peaks: three-quarters for the young pulsars, and $60 \%$ of the MSPs. Figure 21 shows a sample of four recurring profile shapes, which we classify using the ratio $R_{\mathrm{rf}}^{i}$ of the half-widths of the rising and falling peak edges, included in the auxiliary files. Here, the index $i=1,2$ indicates the first and second gamma-ray peaks following phase $\phi=0$. In panel (a) of the figure, the sharpest edges are the trailing edge of the first peak, $R_{\mathrm{rf}}^{1}>1$, and the leading edge of the second 
peak, $R_{\mathrm{rf}}^{2}<1$. In panel (d), it is the opposite: the "outer" peak edges are the most abrupt, $R_{\mathrm{rf}}^{1}<1$ and $R_{\mathrm{rf}}^{2}>1$. In panel (b), the leading edges are sharpest, $R_{\mathrm{rf}}^{1,2}<1$ while for panel (c), the trailing edges are steep, $R_{\mathrm{rf}}^{1,2}>1$. About half of the profiles are classifiable with good statistical significance, and nearly all of these have sharp outer edges as in panel (d). This is the expected pattern for caustics from a hollow cone. A few pulsars depart significantly from this pattern, most prominently those for which both peaks fall sharply ( $\operatorname{panel}(\mathrm{c})$ ). These tend to have $\Delta \approx 0.5$, suggesting emission from both poles. Many MSPs do not fit this simple scheme and cannot be classified in this way with any confidence. More detailed analysis of the pulse width statistics may uncover other trends.

For two of our highest-statistics young pulsars, Vela and J1057-5226, the bridge emission shows persistent structure that can be identified as a third peak. In addition, two MSPs (J1231-1411 and J0751+1807) show significant third peaks. For Vela we have sufficient statistics to see that the phase of the third peak shifts with energy (Abdo et al. 2010n); this is not expected for a simple caustic-induced peak. To explain such structure we must go beyond simple geometrical approximation and employ models with a full treatment of the radiation spectrum and its variation through the magnetosphere (e.g., Du et al. 2011; Wang et al. 2011). As LAT statistics improve, we can expect further such "P3" detections and better constraints on the origin of such pulse components.

Table 12 lists 11 pulsars with significant "M"-type off-peak emission, suggesting the possibility of nearly constant magnetospheric emission. However, several of these pulsars are faint or reside in regions of high and/or complex diffuse background, which complicates the spectral analysis. Therefore, we identify only four young pulsars (PSRs J0633+1746 "Geminga," $\mathrm{J} 1836+5925$, J2021+4026, and J2055+2539) and two MSPs (PSRs J2124-3358 and J2302+4442) that have strong evidence for exponentially cutoff off-peak emission well in excess of the inferred diffuse background (including estimated systematic uncertainty). For the young pulsars this is a serious challenge to outer-magnetosphere models radiating only above the null-charge surface; such weakly pulsed emission should be rare, being expected only for nearly aligned pulsars with $\zeta \approx \pi / 2$. In contrast, lower altitude emission (such as from SG or extended polar cap models; Dyks et al. 2004; Venter et al. 2009) provides a natural explanation for off-peak, noncaustic emission. The remaining five pulsars with "M"-type off-peak emission (J0340+4130, J1124-5916, J1620-4927, J1747-2958, and J1813-1246) should be treated with caution as the uncertainty in the flux from systematic error in distinguishing the diffuse background from magnetospheric emission for these sources makes it difficult to probe faint off-peak emission. However, lower-altitude emission may also be present in a number of these cases. For MSPs, and especially for radioquiet young pulsars, we expect that the magnetic impact angle $|\beta| \equiv|\alpha-\zeta|$ is relatively large, i.e., the Earth line of sight passes far from the radio pole. Thus, off-peak emission for these objects may be associated with large $|\beta|$. The separatrix layer in the wind zone just outside the magnetosphere has also been suggested (Bai \& Spitkovsky 2010) as a site for such gamma-ray flux.

The majority of MSPs (27) have profiles that are very similar to those of young pulsars, with a variety of double and single-peaked profiles with radio lags following the $\delta-\Delta$ trend seen in the normal radio-loud pulsars. A standard SG or OG geometry with narrow gaps can fit these (Venter et al.
2009), requiring surprisingly high-multiplicity pair cascades in these old pulsars. The rest have very different profile types. In particular, a relatively large fraction (6) have phase-aligned radio and gamma-ray pulses, four have $\delta \approx 0.99$ while two (PSRs J0030+0451 and J1810+1744) have $\delta \approx 0.85$ due to the particular method of defining the fiducial radio phase. The peak alignment suggests that the radio as well as the gammaray pulses result from caustic emission at altitudes that are a large fraction of the light cylinder in the relatively small MSP magnetospheres (Venter et al. 2012). The Crab pulsar, with sharp (caustic) main and inter-pulse radio components composed of "giant pulse" emission and aligned with the two gamma-ray pulses, likely represents a similar case. If the radio emission is caustic, a very low level of polarization may result from depolarization of emission over a large range of altitudes (Dyks et al. 2004). In fact, at least four of the six MSPs that have aligned profiles do show little or no linear polarization, as well as unusually steep radio spectra and high $B_{\mathrm{LC}}$ (Venter et al. 2012; Espinoza et al. 2013). There are also MSP profiles (7) where the gamma-ray peaks lead the main radio peak by $\sim 0.2-0.3$ in phase, a profile class that is so far not seen in the young gamma-ray pulsar population. A "pair-starved polar cap" model provides good fits for this class (Venter et al. 2009), implying emission from nearly the full open zone at all altitudes and the same pole that dominates the radio pulse. Three of these MSPs, PSRs J1124-3653, J1744-1134 and J2214+3000, do not have the expected value of $\delta$ in Table 8 ; this is because we chose to use the opposite hemisphere when defining the fiducial radio phase. The best-fit magnetic inclination angles for all of these profile classes show a very wide range, from nearly aligned to nearly orthogonal (T. J. Johnson et al., in preparation). The $|\beta|$ values show a large range since MSPs have relatively larger radio beams than young, shorter-period pulsars, allowing the radio pulse to be visible at larger $\zeta$.

The gamma-ray light curves in this catalog strongly suggest that the gamma-ray emission is distributed in a narrow gap bordering the closed field line boundary. Although the light curves show a large degree of regularity, none of the currently proposed emission models alone is able to account for all of the observed properties. While many light curves follow the OG pattern, some, particularly MSPs and some radioquiet pulsars, do not fit this model and may require additional emission from other zones, such as below the null-charge surface.

\subsection{Luminosity and Spectral Trends}

As in 1PC, the dependence of gamma-ray luminosity on spindown power is one of our most important results. Figures 9 and 10 confirm the general trend of 1PC: young pulsars show increased efficiency for producing gamma rays as the spindown luminosity decreases toward $\dot{E} \approx 5 \times 10^{35} \mathrm{erg} \mathrm{s}^{-1}$, an observation which is in conflict with the two-layer OG model of Takata et al. (2010) that predicts a much flatter trend at high $\dot{E}$. Above $\approx 5 \times 10^{35} \mathrm{erg} \mathrm{s}^{-1}$ MSPs show a similar trend but there is a wide range of efficiencies below this $\dot{E}$. Figure 10 shows this more directly, and emphasizes the point that for most gamma-ray pulsars the apparent efficiency exceeds $10 \%$ and for the lowest $\dot{E}$ efficiencies approach unity. This tension may be slightly mitigated if MSP moments of inertia, and thus spindown luminosities, are a few times larger than the standard $I_{0}=10^{45} \mathrm{~g} \mathrm{~cm}^{2}$ assumed here (Demorest et al. 2010). Nevertheless, the high efficiencies are remarkable, meaning that gamma rays trace the bulk energetics of the pulsar 
machine and implying that studies correlating this output with other observables provide excellent prospects for understanding pulsar magnetosphere physics.

Two factors complicate such studies. The first is the difficulty of obtaining accurate pulsar distances, which we have discussed in Section 4.2. Improvements to the distance determinations (e.g., from additional very long baseline interferometry parallaxes for the radio-bright pulsars, Deller et al. 2011) provides the best hope for eliminating this large uncertainty. An additional challenge is the uncertainty in the geometry-dependent beaming correction $f_{\Omega}$ (Equation (16)). While for most models and viewing geometries this is within a factor of a few of unity, for some situations (e.g., OG geometries at $\dot{E} \approx 10^{34} \mathrm{erg} \mathrm{s}^{-1}$ where $f_{\Omega} \sim 0.1$; Pierbattista et al. 2012) the correction can be quite substantial. Further, physical effects such as $\alpha$-dependent variation of the gap width, changes in the gamma-ray emissivity along the gap, or differences in the detected pulse spectrum can increase the variation of $f_{\Omega}$ beyond simple geometrical factors.

The large scatter in Figures 9 and 10 may well be due to these two factors. Attempts to quantitatively constrain pulsar physics by fitting the luminosity evolution should certainly marginalize over these uncertainties. However, the current sample does provide a greatly improved testbed for comparing predictions of the heuristic $L_{\gamma} \propto \dot{E}^{1 / 2}$ law with more detailed predictions (e.g., Takata et al. 2010).

We can also check the $\dot{E}$ dependence of the spectral fit parameters $\Gamma$ (Figure 7 ) and $E_{\text {cut }}$. As for $1 \mathrm{PC}$ we see that the lowest measured $\Gamma$ are near the limit of $\Gamma=2 / 3$ for singleparticle curvature or synchrotron radiation. In addition, there is a trend toward a softer spectrum (larger $\Gamma$ ) at high spindown luminosity with $\Gamma \propto \dot{E}^{0.2}$. One explanation for this trend is increased pair formation activity in high- $\dot{E}$ pulsars, leading to pair cascades and steep radiating particle spectra. With this catalog we now have the statistics to separately probe the trend in MSPs, which appears steeper with $\Gamma \propto \dot{E}^{0.4}$.

There is no apparent trend of $E_{\text {cut }}$ with $\dot{E}$. If this cutoff follows the radiation-reaction limited energy, and $L_{\gamma} \propto \sqrt{\dot{E}}$, then we expect $E_{\text {cut }} \propto P^{-1 / 4} \dot{E}^{1 / 8}$ so the dependence on spindown power should indeed be weak. One might also expect that MSPs with small $P$ would have larger $E_{\text {cut }}$. However, the small radius of curvature in their compact magnetospheres tends to ensure that the primaries reach lower maximum energy, if radiation-reaction limited.

We note two important caveats to those wishing to use these phase-average spectral fits to constrain models. First, for the fainter pulsars (especially the MSPs) the covariance between $\Gamma$ and $E_{\text {cut }}$ is substantial and the apparent trends may be affected. Second, it is clear from our phase-resolved studies of the brighter pulsars (Abdo et al. 2010c, 2010g, 2010n) that phase-dependent variations in the spectral parameters can be nearly as large as the variations in the full population. Additional evidence for varying $E_{\text {cut }}$ appears when we allow the exponent $b$ to vary in the spectral fits, as discussed in Section 6.1. Nevertheless, nonexponential cutoffs may be present in some cases as discussed in Section 8.4.

\subsection{Pulsar Population and the Millisecond Pulsar Revolution}

As the LAT pulsar sample grows, our ability to make statistically powerful statements about the Galactic neutron stars and their evolution increases. Compared to radio surveys, the LAT provides a new and differently biased sample of the energetic pulsars, so many conclusions drawn from the classic radio samples need to be revisited.

Perhaps the most dramatic progress presented in this catalog is the major increase in the MSP sample from 8 objects to 40, with discoveries continuing (Table 4). In fact, the LAT has proved such an excellent signpost to nearby energetic MSPs that the LAT-guided discoveries represent a large and increasing fraction of the known energetic Galactic MSPs. For example, 70 Galactic (non-globular cluster) MSPs were known before Fermi; there are now 120 such MSPs, 39 of which are in the present catalog. This dominance is especially obvious for $P<3 \mathrm{~ms}$; as noted by Ray et al. (2012) the LAT-detected MSPs are a shorterperiod, more-energetic population than radio-selected MSPs.

Also, Roberts (2013) has noted that Fermi-guided pulsar searches have resulted in a dramatic ten-fold increase in the number of Galactic MSPs in tight binaries with pulsar-driven companion winds, the so-called "black widows" and "redbacks." This is because the gamma-ray signal penetrates the companion wind, flagging the source as a possible MSP and guiding repeated radio searches for the intermittently visible radio pulsations.

In addition to the Galactic MSPs, there is a population of some 120 pulsars in the globular cluster system, discovered through radio searches. As these clusters are relatively distant it is not surprising that, to date, the LAT has detected pulsed signals from only two of the youngest, most energetic cluster MSPs (Freire et al. 2011; Johnson et al. 2013). However, the detection of gamma-ray sources coincident with a dozen globular clusters (Abdo et al. 2010a; Kong et al. 2010; Tam et al. 2011) indicates a substantial MSP population (first predicted by Chen 1991). Indeed, the cluster gamma-ray flux appears to correlate with the expected MSP formation rate (Abdo et al. 2010a). It had been noticed that a large fraction of the radio MSPs in globular clusters are tight, often evaporating, black-widow-type binaries and it was suggested that this was a true difference from the Galactic field population (King et al. 2003). However, it now seems that this was largely an artifact of the very long radio dwell times used for cluster searches that allowed discovery of radio-intermittent MSPs generally undetectable to field surveys. With the LAT unassociated sources providing a signpost for deep targeted searches, a similar field black widow population has now been discovered. Thus, the LAT has uncovered a new sample of MSPs with less (or at least different) bias than the classical radio population, demanding a re-assessment of the MSP population and its evolution.

For young pulsars, there is good hope that the LAT can similarly provide a much more complete census of massive star remnants in the nearby Galaxy. For example, Watters \& Romani (2011) found that the 1PC sample implied an energetic young pulsar birthrate of $1.69 \pm 0.24$ pulsars $/ 100 \mathrm{yr}$, a substantial fraction of the 2.4/100 yr OB star birthrate and the $2.30 \pm 0.48$ $\mathrm{SNe} / 100 \mathrm{yr}$ rate (Li et al. 2011). This catalog will allow further refinement of this comparison.

Finally, we conclude by noting at least one expected pulsar population remains missing from the present catalog. As in 1PC, no pulsed detection of a young spin-powered object in a massive binary has yet been made. Several percent of the pulsar population is expected to pass through this channel (the progenitor of the double neutron star binaries). A few such pulsars are known from the radio, but like PSR B1259-63 are detected only at large orbital separation. For short period systems, plasma from the companion wind presumably prevents radio pulsar detection. However, gamma rays provide 


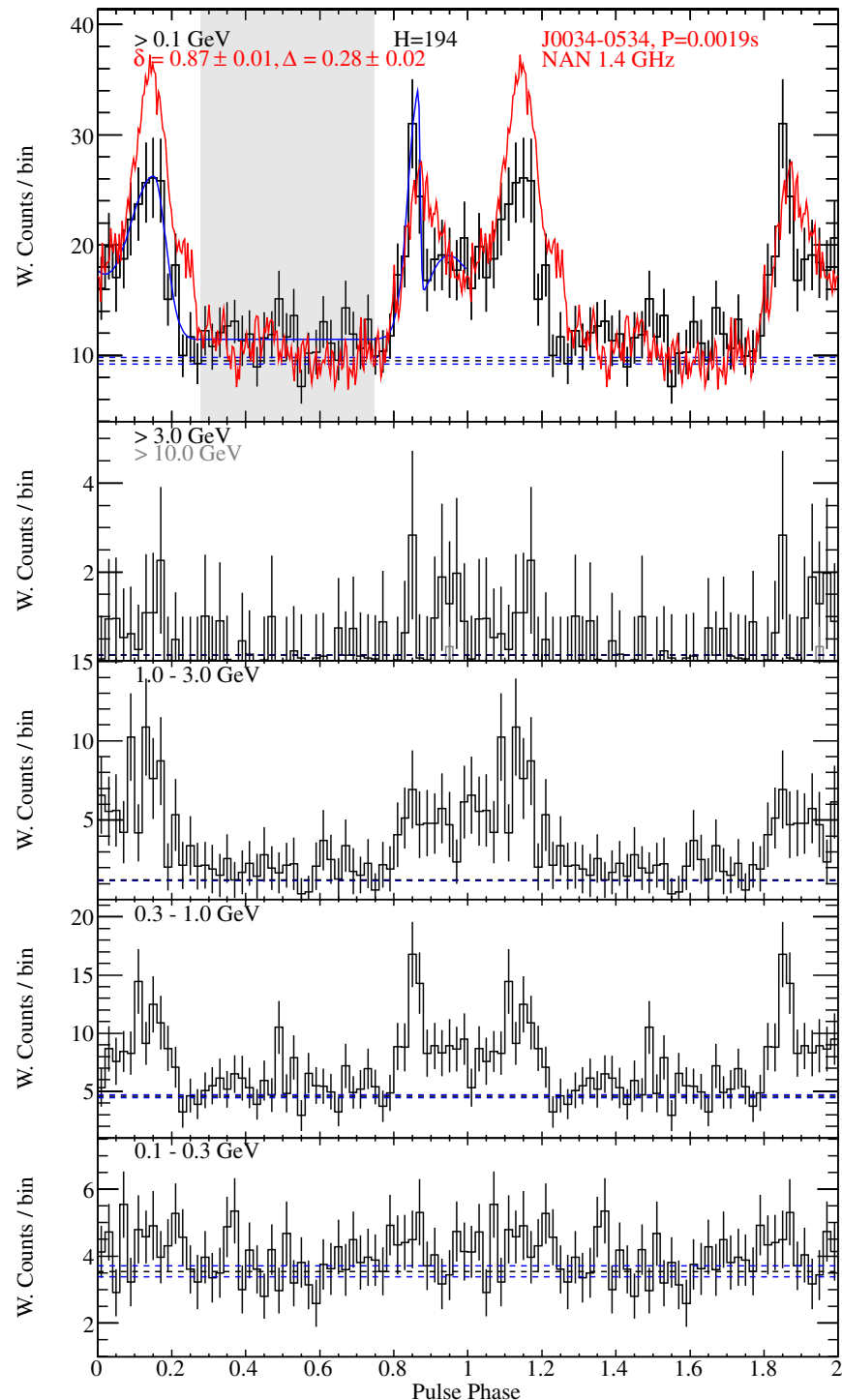

(a)

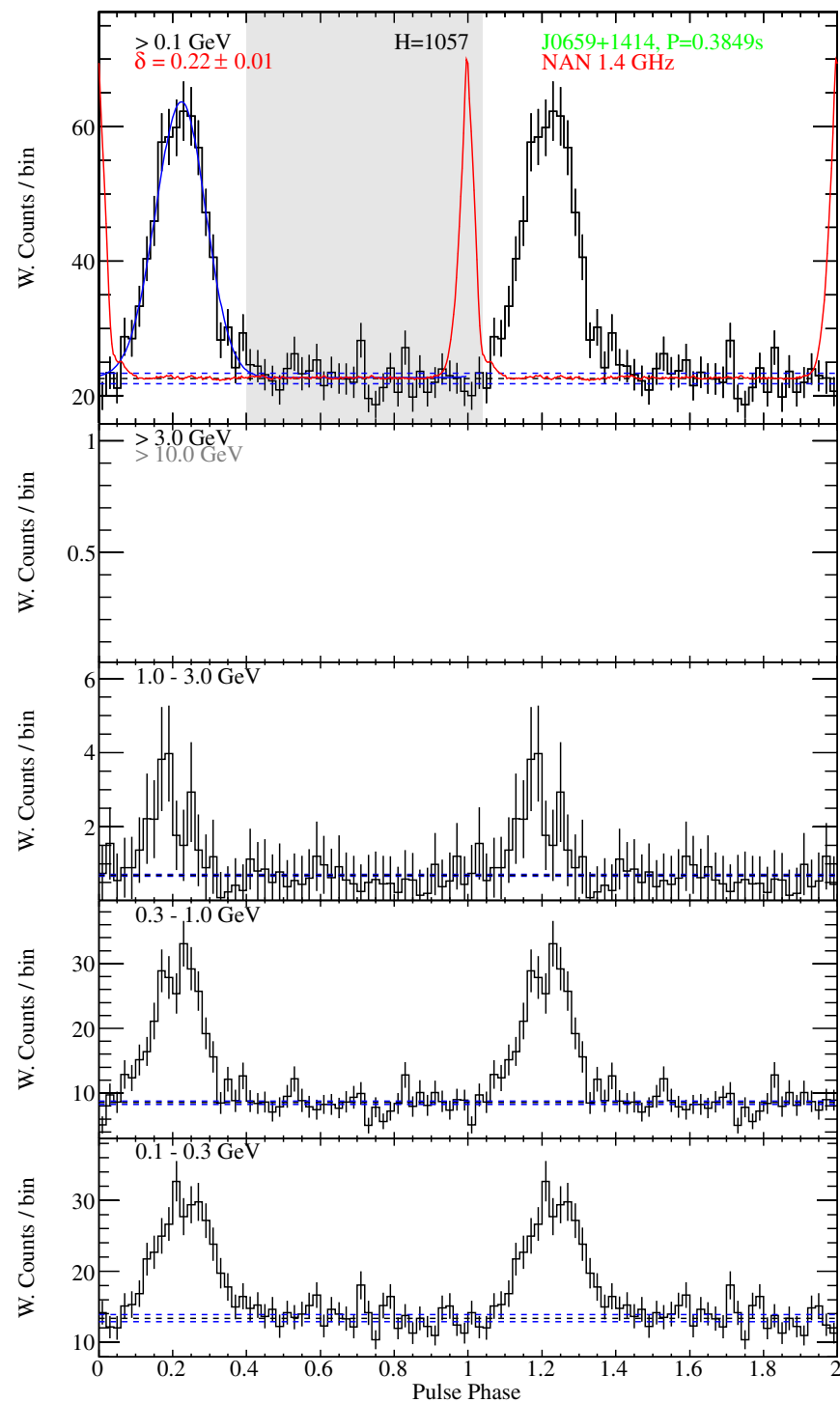

(b)

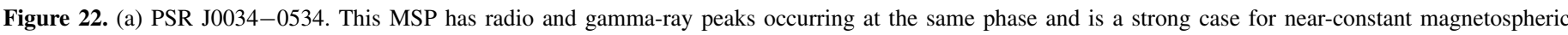

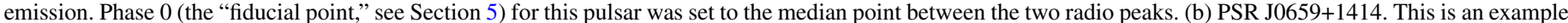

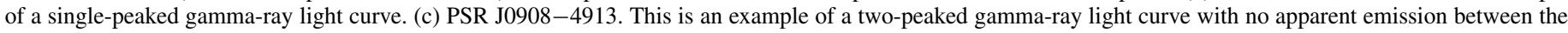

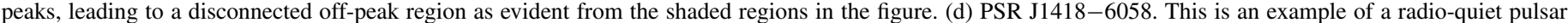

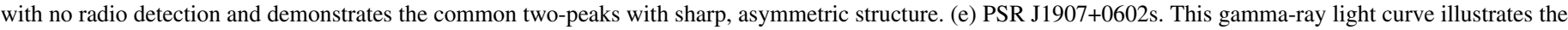

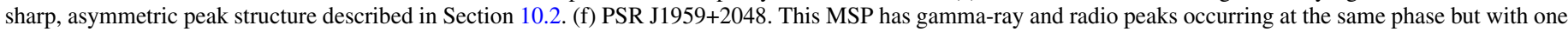

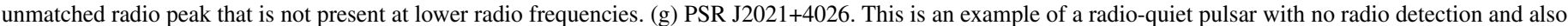

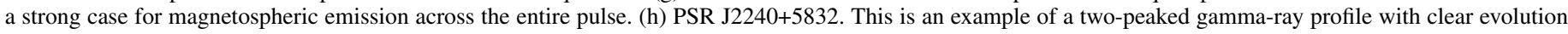
of the P2/P1 ratio with energy.

(A color version and the complete figure set (117 images) are available in the online journal.)

an excellent signpost to such wind-absorbed pulsars as witnessed by recent success in detecting short-period black widows and redbacks in LAT sources. It is probable that some of the gamma-ray detected massive binaries host spin-powered pulsars, a possibility discussed by Dubus (2006). With improved search techniques, such objects may appear in future LAT pulsar catalogs.

The Fermi-LAT Collaboration acknowledges generous ongoing support from a number of agencies and institutes that have supported both the development and the operation of the LAT as well as scientific data analysis. These include the National Aeronautics and Space Administration and the Department of Energy in the United States; the Commissariat à l'Energie Atomique and the Centre National de la Recherche Scientifique/Institut National de Physique Nucléaire et de Physique des Particules in France; the Agenzia Spaziale Italiana and the Istituto Nazionale di Fisica Nucleare in Italy; the Ministry of Education, Culture, Sports, Science and Technology (MEXT), High Energy Accelerator Research Organization (KEK), and Japan Aerospace Exploration Agency (JAXA) in Japan; and the K. A. Wallenberg Foundation, the Swedish Research Council, and the Swedish National Space Board in Sweden. 


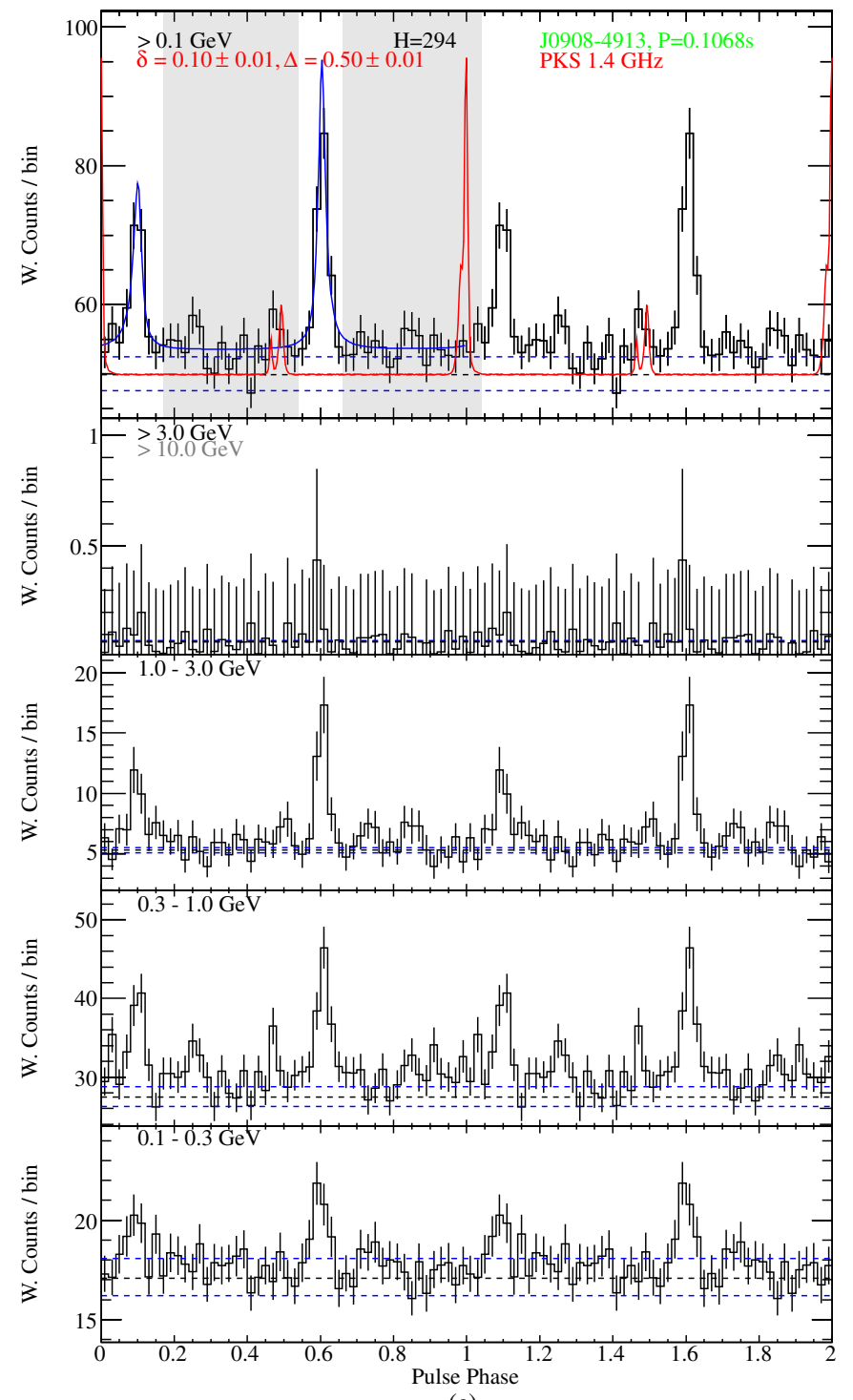

(c)

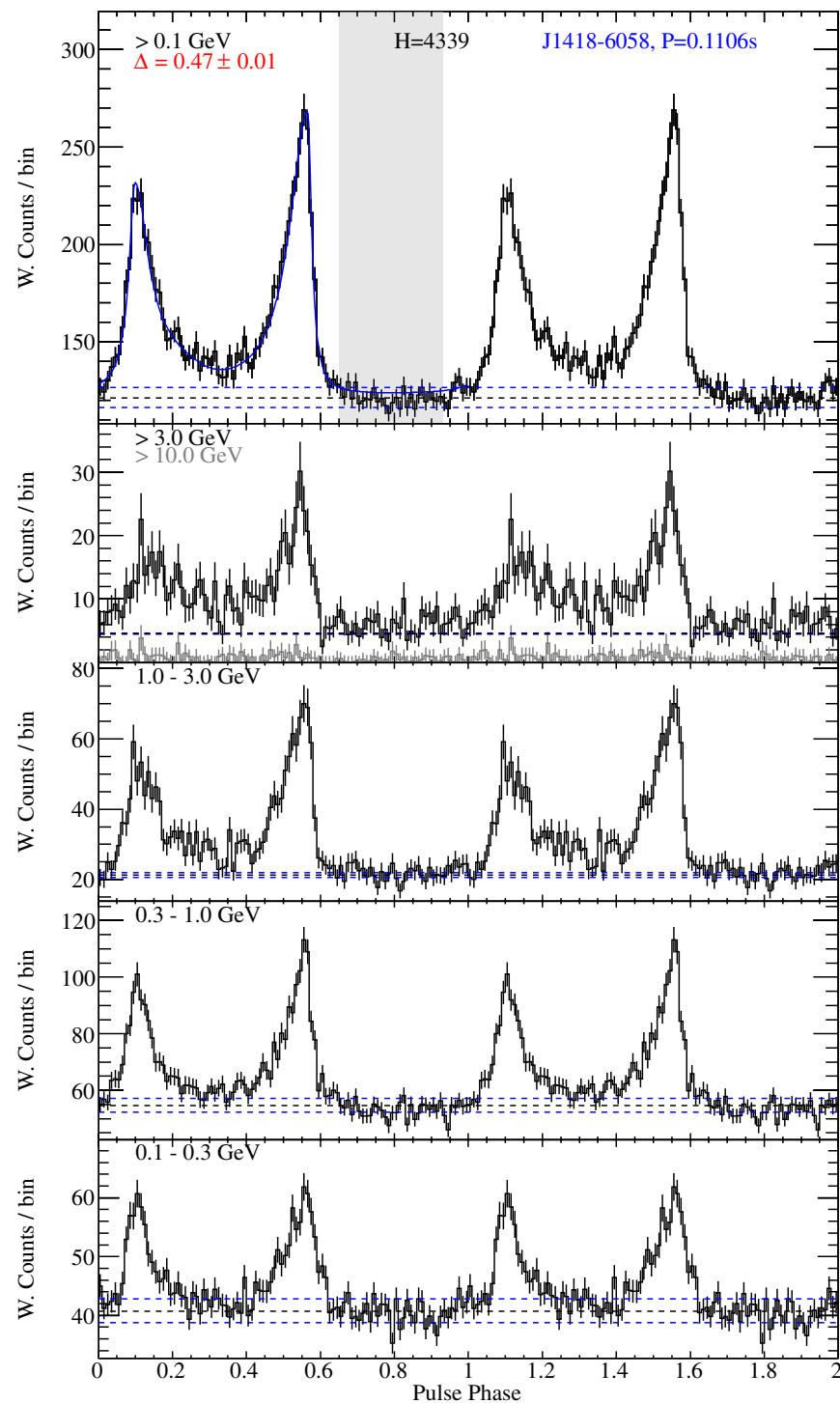

(d)

Figure 22. (Continued)

Additional support for science analysis during the operations phase is gratefully acknowledged from the Istituto Nazionale di Astrofisica in Italy and the Centre National d'Études Spatiales in France.

The Parkes Radio Telescope is part of the Australia Telescope which is funded by the Commonwealth Government for operation as a National Facility managed by CSIRO. The Green Bank Telescope is operated by the National Radio Astronomy Observatory, a facility of the National Science Foundation operated under cooperative agreement by Associated Universities, Inc. The Arecibo Observatory is part of the National Astronomy and Ionosphere Center (NAIC), a national research center operated by Cornell University under a cooperative agreement with the National Science Foundation. The Nançay Radio Observatory is operated by the Paris Observatory, associated with the French Centre National de la Recherche Scientifique (CNRS). The Lovell Telescope is owned and operated by the University of Manchester as part of the Jodrell Bank Centre for Astrophysics with support from the Science and Technology Facilities Council of the United Kingdom. The Westerbork Synthesis Radio Telescope is operated by Netherlands Foundation for Radio
Astronomy, ASTRON. This work made extensive use of the ATNF pulsar catalog (Manchester et al. 2005).

\section{APPENDIX A}

\section{SAMPLE LIGHT CURVES AND SPECTRA}

Figures 22(a)-(h) show sample multiband light curves. Figures 23(a)-(i) show sample gamma-ray spectra. Plots for all pulsars in this catalog are available in the supplemental online material, detailed in Appendix B.

For each pulsar, the top frame of the light curve figure shows the 0.1 to $100 \mathrm{GeV}$ gamma-ray light curve, with the same data repeated over two rotations, to clarify structures near phases 0 and 1 . The pulsar name follows the same color-code as the markers in the plots in the body of the text: red for MSPs; blue for young radio-quiet, and green for young loud. The letter $P$ gives the rotation period. The letter $H$ gives the $H$-test value for the gamma-ray light curve in the top frame. The letters $d$ and $D$ give the lag $\delta$ of the first gamma peak relative to the radio fiducial phase, and the separation $\Delta$ of the outermost gamma peaks, respectively. The dark (blue online) curve over the phase 

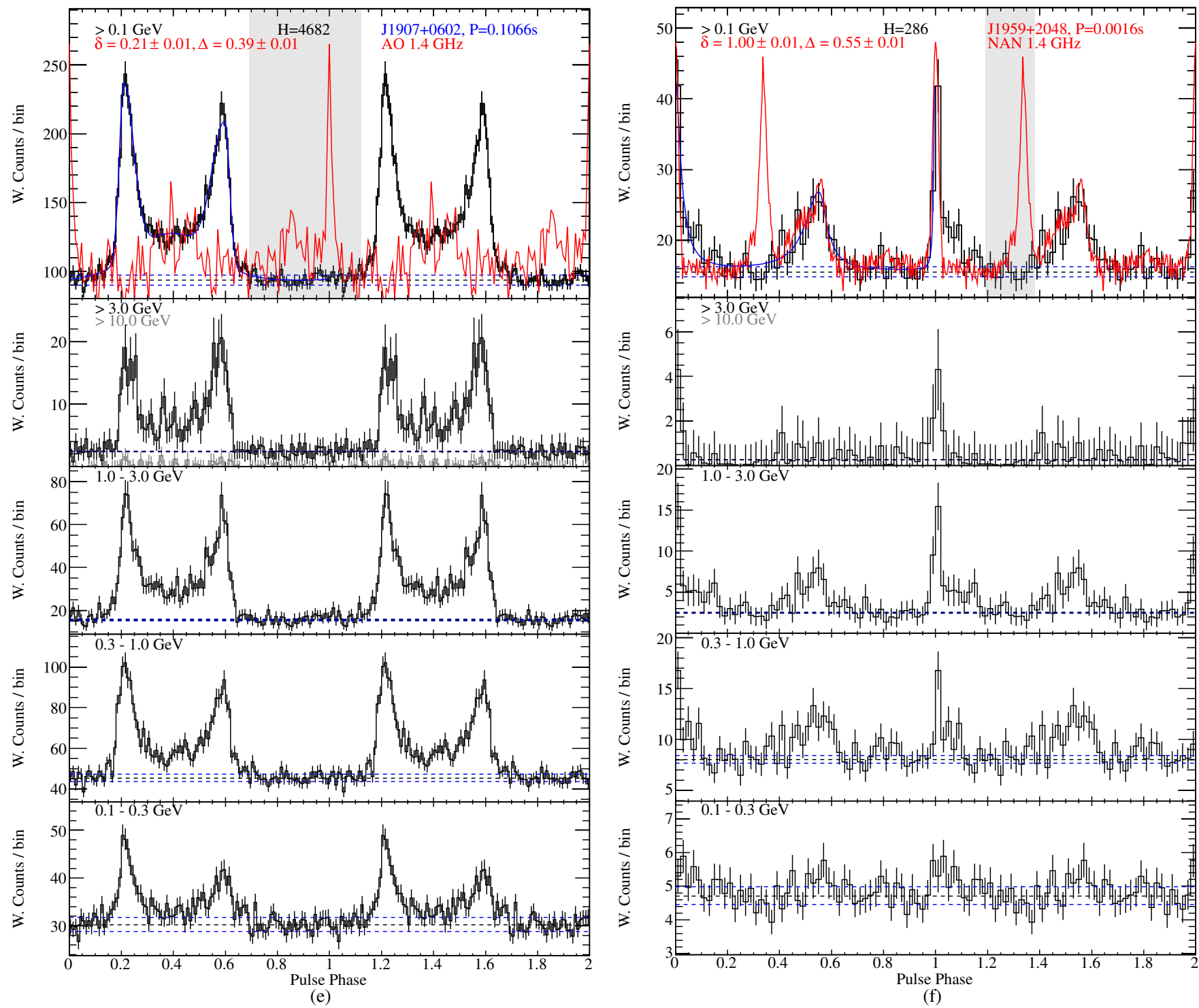

Figure 22. (Continued)

range $\phi \in[0.0,1.0)$ in the top frame is the 0.1 to $100 \mathrm{GeV}$ profile fit described in Section 5. The radio profile, when it exists, is drawn (red curve online) in the top frame, also repeated over two rotations, with the observing frequency as indicated on the figure. The radio telescopes that provided the profiles shown are indicated by NAN (Nançay), PKS (Parkes), JBO (Jodrell Bank), AO (Arecibo), GBT (Green Bank), and WSRT (Westerbork). The shaded gray region in the top frame represents the off-peak interval as defined in Section 7.1. The lower frames show the gamma-ray light curves in the indicated energy bands. The horizontal, dashed lines indicate the estimated background levels, for the gamma-ray light curves, and the associated uncertainties as described in Section 5.

For each pulsar, the gamma-ray spectral points are from individual energy-band fits in which the pulsar spectrum is approximated as a pure power law. For energy bands in which the pulsar is detected with TS $<9$ we report $95 \%$ confidence level upper limits. The solid black line corresponds to the bestfit PLEC1 model from the full energy range fit, while the red (online) dashed lines represent the $1 \sigma$ confidence region on the best-fit model. For pulsars where a pure exponential cutoff $(b=1)$ is disfavored $\left(\mathrm{TS}_{\mathrm{b} \text { free }} \geqslant 9\right)$ we also show the PLEC fit (blue online).

\section{APPENDIX B}

\section{DESCRIPTION OF THE ONLINE CATALOG FILES}

The results described in this paper are provided in the supplemental online material. In addition, a compressed ("gzip") electronic archive file ("tar") called 2PC_auxiliary_files_v\#\#.tgz is available at http://fermi.gsfc.nasa.gov/ssc/data/access/lat/2nd_ PSR_catalog/. The archive contains a directory structure with FITS tables of the analysis results, images of the light curves and spectra for each pulsar, and individual FITS files for each pulsar with the light curves and spectra in numerical form. The rotation ephemeris for each pulsar is in a TEMPO2 parameter (".par") text file.

Additional information, such as, for example, the half-widths at half-maximum of the leading and trailing edges of the peak fits described in Section 5, and their uncertainties, is also provided. Another example is that in addition to the exponentially cut-off power law spectral parameters listed in 


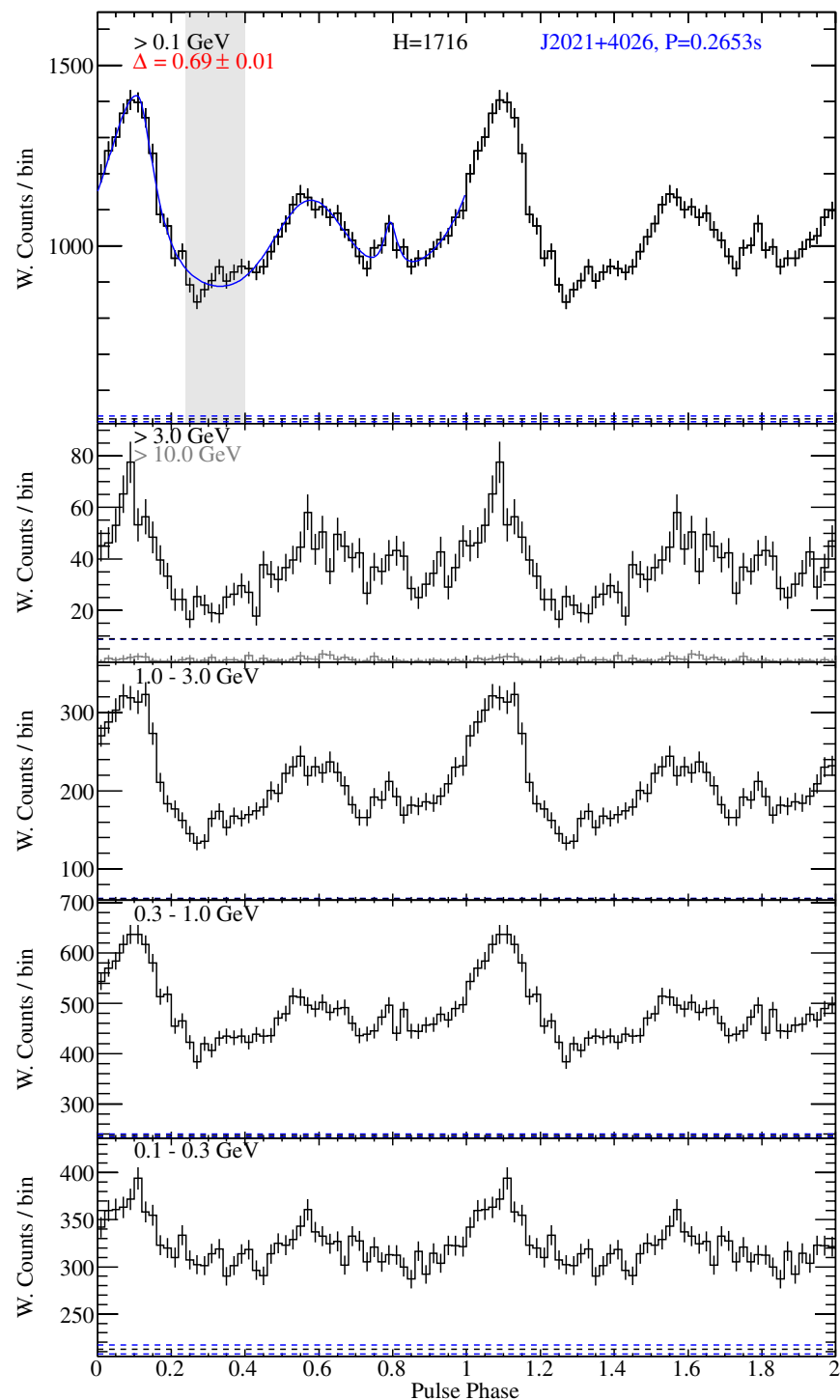

(g)

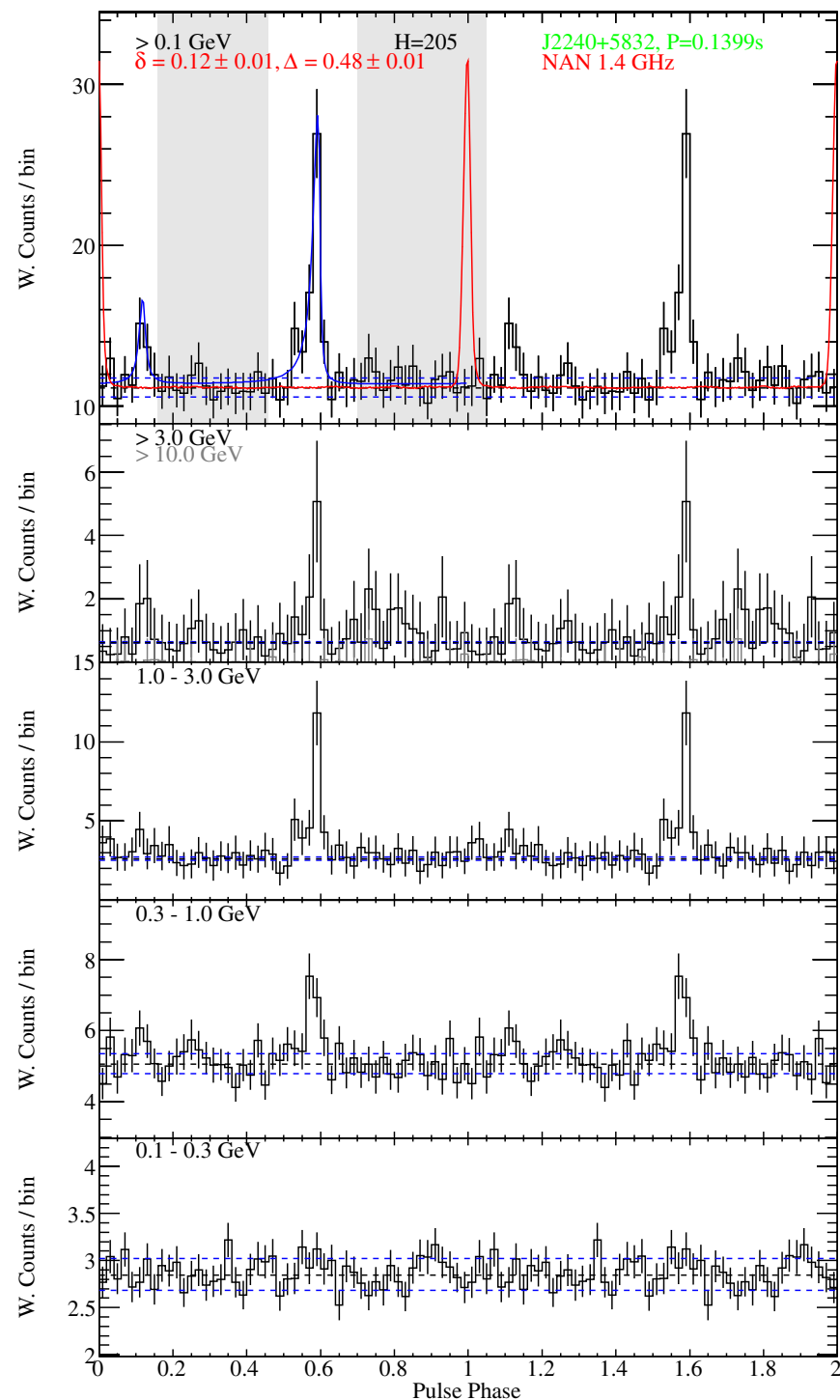

(h)

Figure 22. (Continued)

Tables 9 and 10, we provide the results of the pure powerlaw fits, and of the fits with the $b$ parameter free for pulsars with $\mathrm{TS}_{\mathrm{b} \text { free }} \geqslant 9$ (see Section 6). Detailed column descriptions for the main FITS tables are in Appendix B.1, and detailed column descriptions for the individual pulsar FITS tables are in Appendix B.2.

\section{B.1. Detailed Column Descriptions of Main Catalog FITS Tables}

The main catalog file, 2PC_catalog_v\#\#.fits contains summary results for all 117 pulsars. The file contains four FITS table extensions: PULSAR_CATALOG, SPECTRAL, OFF_PEAK, and REFERENCES. The primary extension is empty. Table 18 details the contents of the PULSAR_CATALOG extension. This file is also duplicated in ascii format (2PC_catalog_v\#\#.asc) with the four tables appended in the order listed above in the file 2PC_auxiliary_files_v\#\#.tgz available at http://fermi.gsfc.nasa.gov/ssc/data/access/lat/2nd_PSR_ catalog/.

The PULSAR_CATALOG extension contains one row for each pulsar with most of the information provided in the ta- bles in this paper, as well as some additional quantities that can be computed from these results. Exceptions are the complete spectral analysis (reported in the SPECTRAL extension; Table 19), and the results of the off-peak analysis (reported in the OFF_PEAK extension; Table 20).

The SPECTRAL extension (Table 19) contains the results of the spectral analysis, one row for each pulsar. Models used in the spectral analysis are PLEC1, PLEC, and PL. The spectral analysis is described in Section 6.1.

The Prefactor, Scale, Photon_Index, and Cutoff values for each pulsar using the PLEC1 model are provided. Results from the fit using the PLEC spectral model are only reported when $\mathrm{TS}_{\mathrm{b} \text { free }} \geqslant 9$. The differential spectrum of the PLEC spectral model (PLSuperExpCutoff) is defined as:

$$
\begin{aligned}
\frac{d N}{d E}= & \text { Prefactor }\left(\frac{E}{\text { Scale }}\right)^{\text {-Photon_Index }} \\
& \times \exp \left\{-\left(\frac{E}{\text { Cutoff }}\right)^{\text {Exponential_Index }}\right\},
\end{aligned}
$$




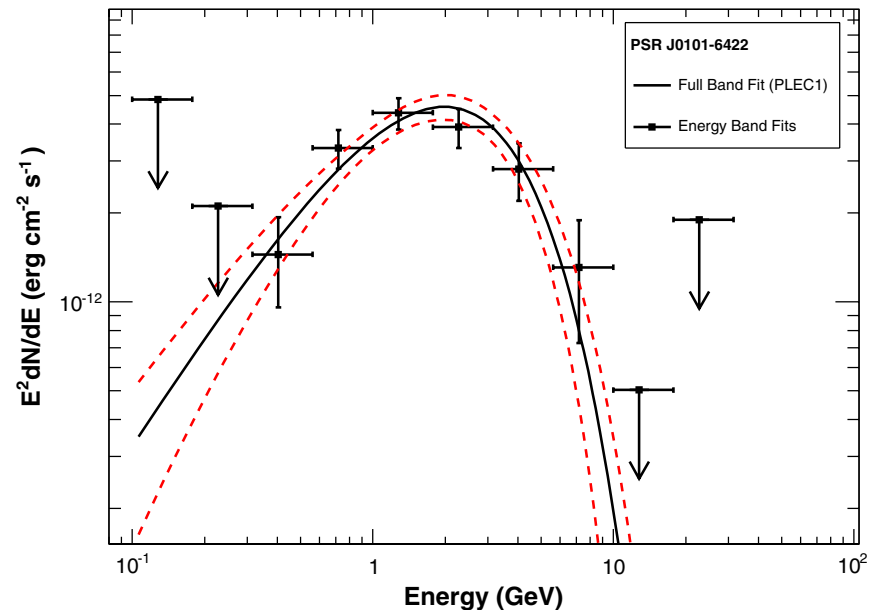

(a)

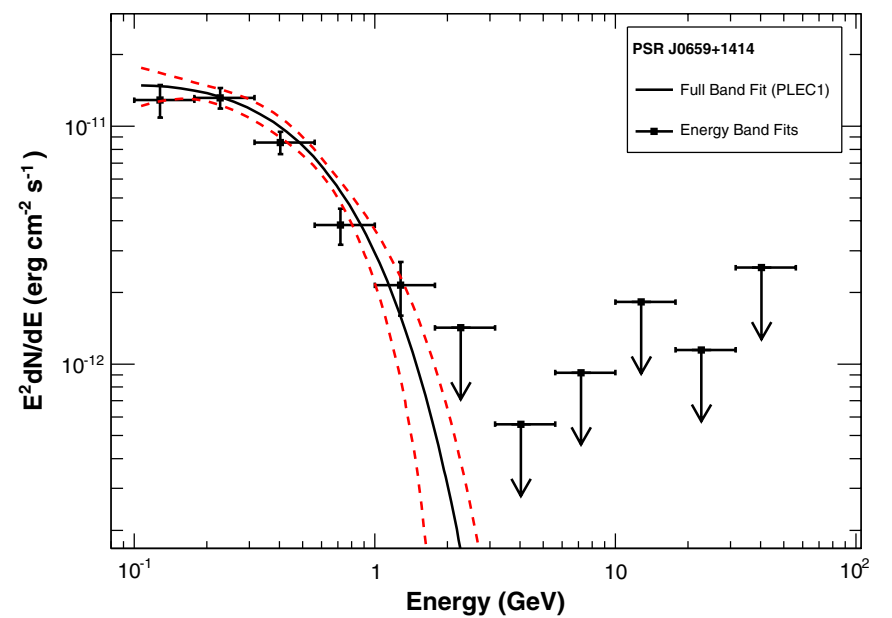

(c)

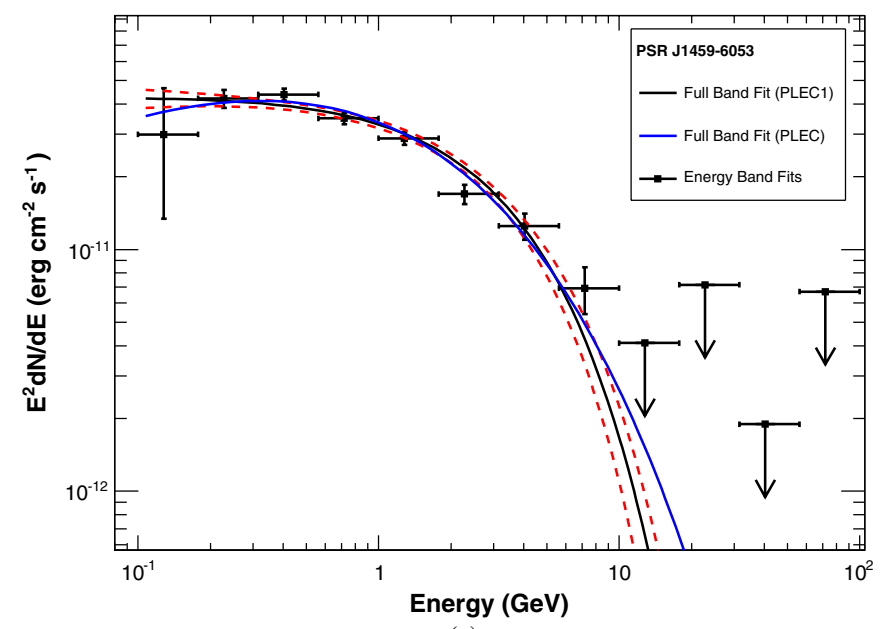

(e)

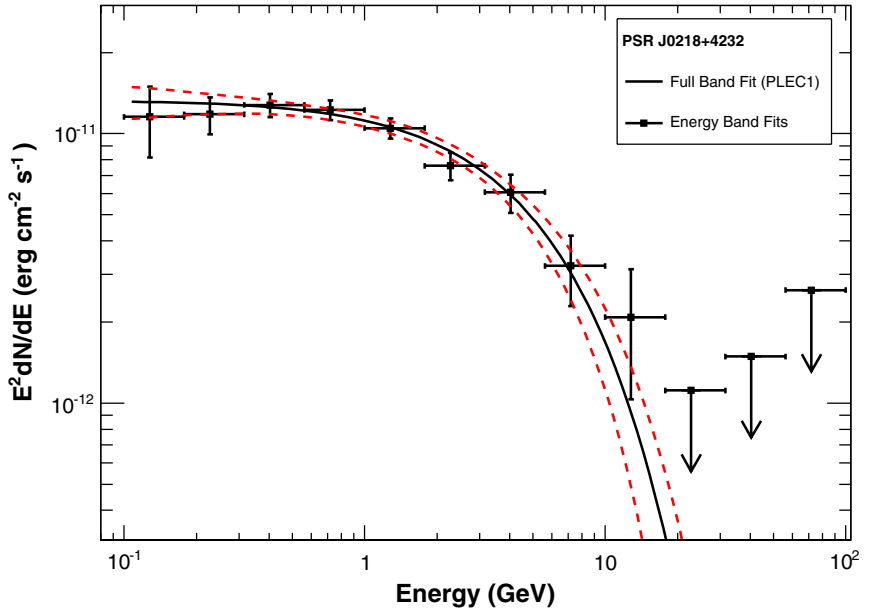

(b)

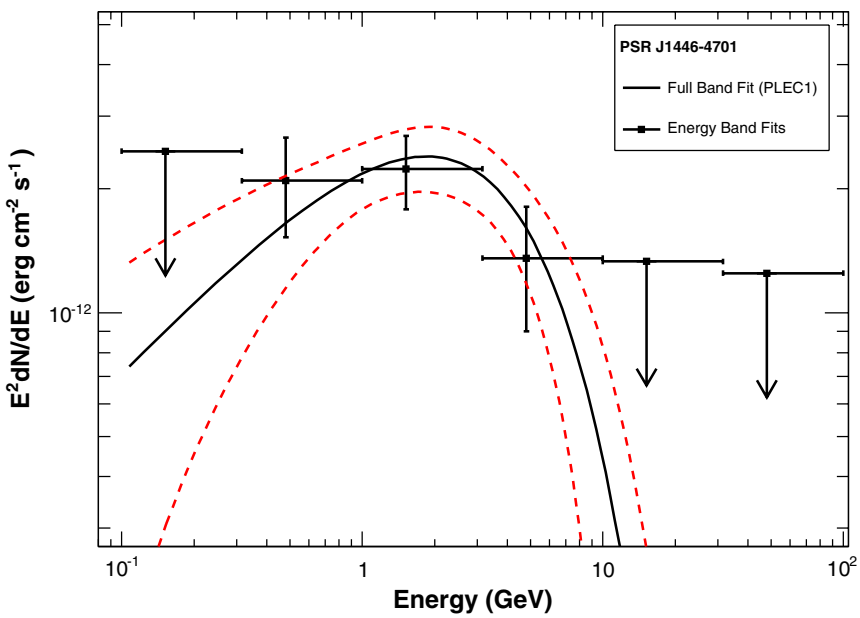

(d)

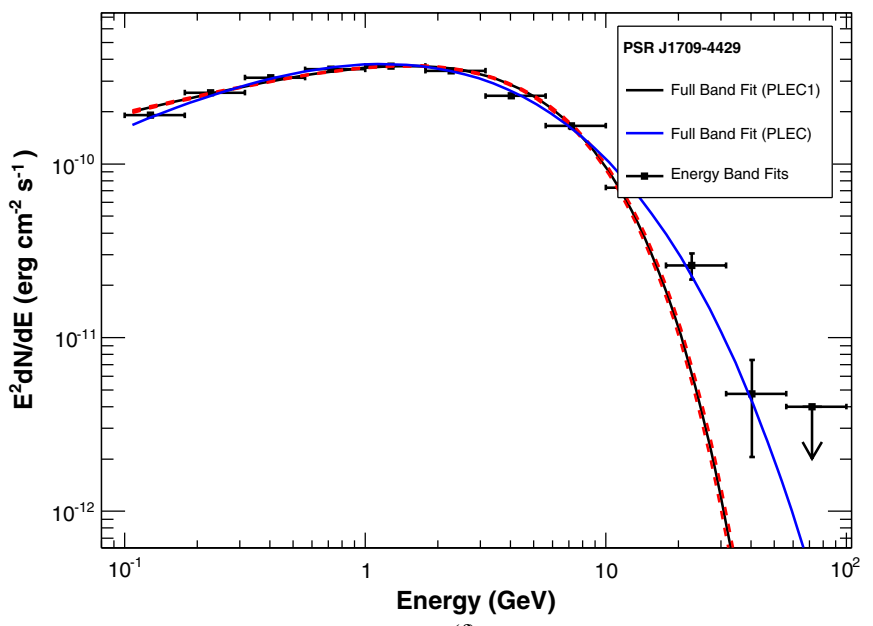

(f)

Figure 23. (a) PSR J0101-6422. This spectrum demonstrates that pulsars are typically most significant near $1 \mathrm{GeV}$ and often cannot be detected as point sources near $0.1 \mathrm{GeV}$. (b) PSR J0218+4232. This spectrum has a cutoff on the high-energy tail of the $E_{\text {cut }}$ distribution. (c) PSR J0659+1414. This spectrum has a cutoff on the low-energy tail of the $E_{\text {cut }}$ distribution. (d) PSR J1446-4701. This is a faint pulsar with only a few points in the spectrum. (e) PSR J1459-6053. For this pulsar, $\mathrm{TS}_{\mathrm{b}}$ free $\geqslant 9$ and the PLEC fit (exponential cutoff parameter not fixed to $b=1$ ) is shown (blue in the online version). The full energy range maximum likelihood fits are, typically, a better measure of the spectrum than fits to the points. (f) PSR J1709-4429. This spectrum illustrates deviations from the PLEC1 model above $1 \mathrm{GeV}$ (PLEC fit shown in blue online). (g) PSR J1713+0747. This is a faint pulsar with a reliable spectral fit and significant emission out to at least a few GeV. (h) PSR J1747-4036. This spectrum is an example of a faint pulsar for which significant spectral curvature cannot be seen with 3 yr of data. (i) PSR J1954+2835. This spectrum has a photon index on the soft (larger) end of the $\Gamma$ distribution. For this pulsar, $\mathrm{TS}_{\mathrm{b}}$ free $\geqslant 9$ and the PLEC fit (exponential cutoff parameter not fixed to $b=1$ ) is shown (blue in the online version).

(A color version and the complete figure set (107 images) are available in the online journal.) 


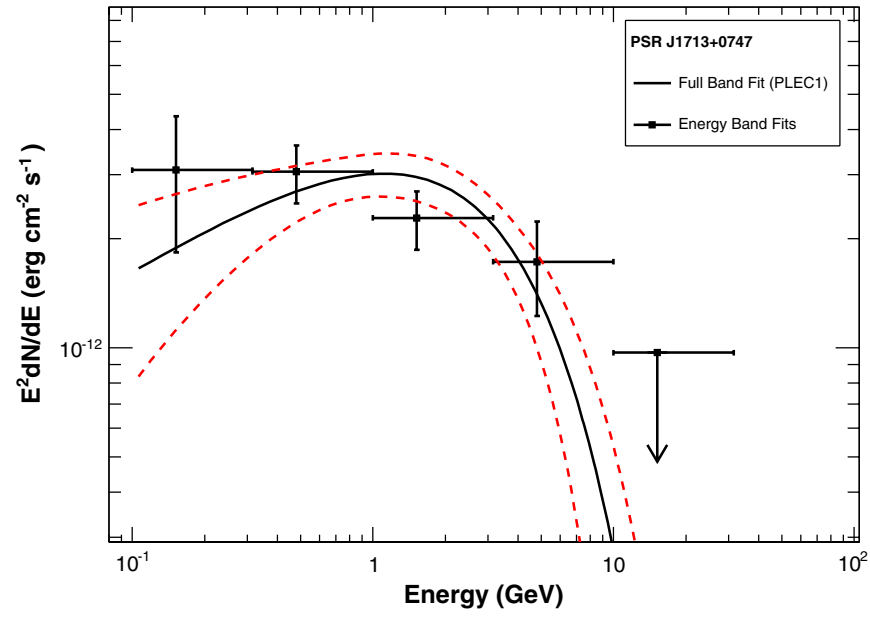

(g)

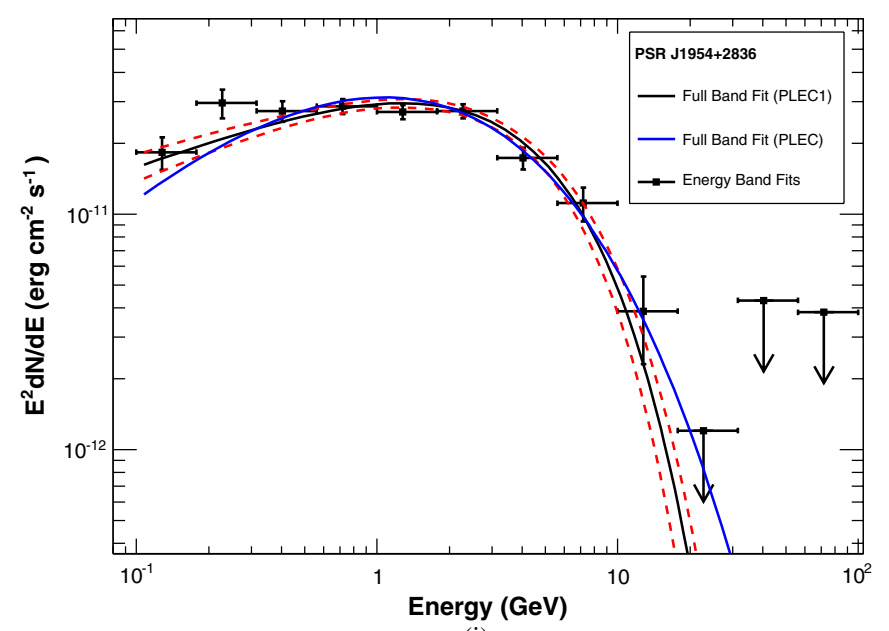

(i)

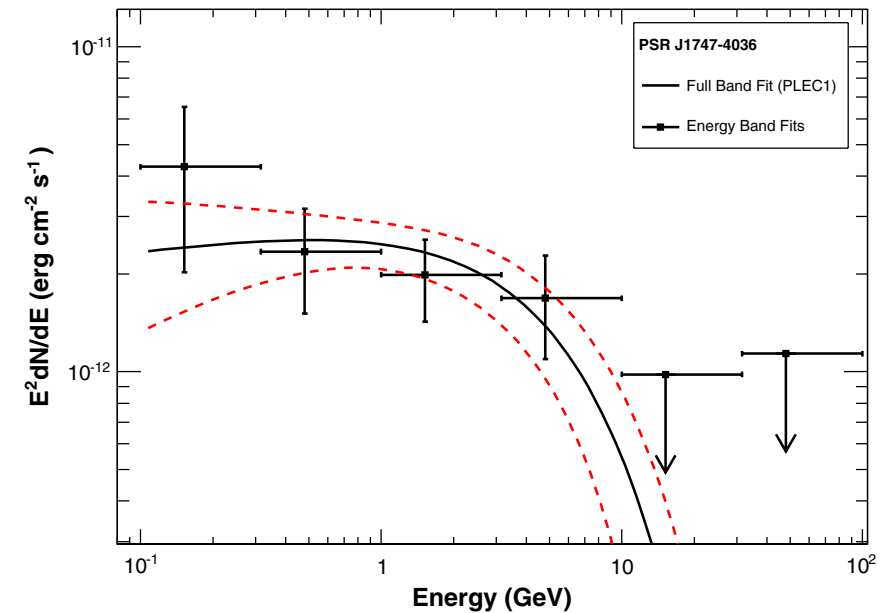

(h)

Figure 23. (Continued)

while the differential spectrum of the PLEC1 model is Equation (B1) with Exponential_Index fixed to 1. When the PLEC fit is reported in the SPECTRAL extension, it includes the value for the Exponential_Index

The differential spectrum of the PL (PowerLaw) spectral model is defined as:

$$
\frac{d N}{d E}=\operatorname{Prefactor}\left(\frac{E}{\text { Scale }}\right)^{- \text {Photon_Index }}
$$

with the Prefactor, Scale, and Photon_Index for each pulsar using the PL model given in the SPECTRAL extension.

The OFF_PEAK extension (Table 20) contains the spatial and spectral results of the search for off-peak emission. The table contains one row for each pulsar. Details of this analysis are given in Section 7.2.

For sources reported with a PL spectral model, the differential spectrum is defined as:

$$
\frac{d N}{d E}=\text { Prefactor } \_\mathrm{OP}\left(\frac{E}{\text { Scale_OP }_{\perp}}\right)^{- \text {Index_OP }}
$$

The Prefactor_OP, Index_OP, and Scale_OP are given in the OFF_PEAK extension.
For sources reported with a PLEC1 spectral model (PLSuperExpCutoff), the differential spectrum is defined as:

$$
\begin{aligned}
\frac{d N}{d E}= & \text { Prefactor_OP }\left(\frac{E}{\text { Scale_OP }}\right)^{- \text {Index_OP }} \\
& \times \exp \left(-\frac{E}{\text { Energy_Cutoff_OP }}\right)
\end{aligned}
$$

and the Prefactor_OP, Index_OP, Scale_OP, and Energy_Cutoff_OP are given in the OFF_PEAK extension as described below.

For the Crab Nebula and Vela-X, we took the spectral shape and initial normalization from Buehler et al. (2012) and Grondin et al. (2013), respectively, and fit only a multiplicative offset (see Section 7.2). For these two sources, the differential spectrum was defined as:

$$
\frac{d N}{d E}=\text { Normalization_OP }\left.\frac{d N}{d E}\right|_{\text {file }}
$$

and Normalization_OP is provided in the OFF_PEAK extension of the main pulsar catalog FITS file.

References used for pulsar distances and radio flux values have been assigned a number, and the number is reported in the PULSAR_CATALOG extension. The REFERENCES extension (Table 21) provides the full information for each reference. 
Table 18

LAT Second Pulsar Catalog FITS Format: PULSAR_CATALOG Extension

Name
PSR_Name
RAJ2000, DEJ2000
GLON, GLAT
Period
P_Dot
E_Dot
F100, Unc_F100
G100, Unc_G100
TS_DC
TS_Cutoff
TS_bfree
Photon_Index, Unc_Photon_Index
Putoff, Unc_Cutoff
Er_Dot_Int, Neg_Unc_P_Dot_Int, Pos_Unc_P_Dot_Int
Pistance, Neg_Unc_Distance, Pos_Unc_Distance
UL_Luminosity
Distance_Method,

Efficiency, Unc_Efficiency, Neg_Sys_Efficiency, Pos_Sys_Efficiency

UL_Efficiency

S1400

UL_S1400

S1400_Ref

Num_Peaks

Shift_Method

Radio_Lag, Unc_Radio_Lag

Units Description

$\begin{array}{ll}\ldots & \text { Pulsar name } \\ \text { deg } & \text { The pulsar position in celestial coordinates (J2000). }\end{array}$

deg The pulsar position in galactic coordinates.

$\mathrm{ms} \quad$ The pulsar rotation period.

$\mathrm{s} \mathrm{s}^{-1} \quad$ The period first derivative, uncorrected for Shklovskii effect or Galactic acceleration; see Section 4.3.

$\mathrm{erg} \mathrm{s}^{-1} \quad$ The pulsar spin-down luminosity, uncorrected for Shklovskii effect or Galactic acceleration; see Section 4.3.

$\mathrm{ph} \mathrm{cm}^{-2} \mathrm{~s}^{-1}$ The best-fit photon flux and statistical error, integrated from $100 \mathrm{MeV}$ to $100 \mathrm{GeV}$. NULL values indicate unreliable spectral fits.

erg $\mathrm{cm}^{-2} \mathrm{~s}^{-1}$ The best-fit energy flux and statistical error, integrated from $100 \mathrm{MeV}$ to $100 \mathrm{GeV}$. NULL values indicate unreliable spectral fits.

... The test statistic obtained at the position of the pulsar, assuming a PLSuperExpCut off spectral model with the exponential index fixed to 1 . The fit uses data from $100 \mathrm{MeV}$ to $100 \mathrm{GeV}$, and includes all pulse phases except as noted in Tables 9 and 10.

.. The significance of the spectral cutoff, obtained from the improvement in $\log$ (Likelihood) from the PLSuperExpCut of f spectral model fit over the PowerLaw spectral fit.

... The improvement in the test statistic when the exponential index is left free in the PLSuperExpCut off spectral fit. If there is no improvement, or the fit is worse, this value is zero.

.. The best-fit photon index and statistical error for the PLSuperExpCutoff spectral model. NULL values indicate unreliable spectral fits.

$\mathrm{MeV}$ The best-fit cutoff energy and the statistical error for the PLSuperExpCutoff spectral model. NULL values indicate unreliable spectral fits.

pc The pulsar's distance measurement and its uncertainties. NULL values indicate that only an upper limit has been determined.

pc Upper limit on pulsar distance when no measured value has been determined, NULL values indicate that a distance measurement has been determined.

The method used to determine the pulsar's distance. Methods are: $\mathrm{K}$ for the kinematic model, DM for the dispersion measure using the NE2001 model of Cordes \& Lazio (2002), 0 for optical measurements, and X for X-ray measurements. DMM means that the distance to the Galaxy's edge, as determined by the maximum DM value provided by the NE2001 model for that line of sight, is taken as an upper limit.

Numerical reference for the distance measurement. The full reference is in the REFERENCES extension of this FITS file.

mas $\mathrm{yr}^{-1} \quad$ The proper motion and errors for the pulsar when available.

... Numerical reference for the proper motion measurement. The full reference is in the REFERENCES extension of this FITS file.

$\mathrm{s} \mathrm{s}^{-1} \quad$ The intrinsic P-dot and associated errors, after contributions from the Shklovskii effect and Galactic acceleration have been removed, see Section 4.3.

$\mathrm{erg} \mathrm{s}^{-1} \quad$ The intrinsic spin down power and associated errors.

$\mathrm{erg} \mathrm{s}^{-1} \quad$ The pulsar luminosity, statistical error, and systematic errors. Systematic errors are derived from the distance uncertainty. Values are NULL when only an upper limit exists.

$\mathrm{erg} \mathrm{s}^{-1} \quad$ Upper limit on the luminosity when no value has been determined. Entries are NULL when a value has been determined. NULL values in all Luminosity columns indicate unreliable spectral fits.

percent The pulsar efficiency, statistical error, and systematic errors from the distance measurement. Values are NULL when only an upper limit has been determined.

percent Upper limit on the pulsar efficiency. Entries are NULL when a value has been determined. NULL values in all Efficiency columns indicate unreliable spectral fits.

mJy Radio flux density at $1400 \mathrm{MHz}$. In some cases, documented in Section 4.1, this value is extrapolated from measurements at other frequencies. Entries are NULL when only an upper limit has been reported.

Upper limit on the radio flux density at $1400 \mathrm{MHz}$ when no measurement has been reported. Entries are NULL when a value has been reported.

Numerical reference for the radio flux density measurement. The full reference is in the REFERENCES extension of this FITS file.

$\begin{array}{ll}\ldots & \text { Number of peaks in the gamma-ray profile. } \\ \ldots & \text { Method used to choose the radio fiducial phase. Methods are: } \mathrm{p} \text { for the peak radio }\end{array}$ intensity, $\mathrm{h}$ for an opposite hemisphere shift ( 0.5 phase shift from the peak intensity), $\mathrm{s}$ for the point of symmetry in the radio profile, and o for some other method as described in the text (used only for PSR J0534+2200).

Phase separation $(\delta)$ between the first gamma-ray peak and the radio peak, and the associated error on that separation. 
Table 18

(Continued)

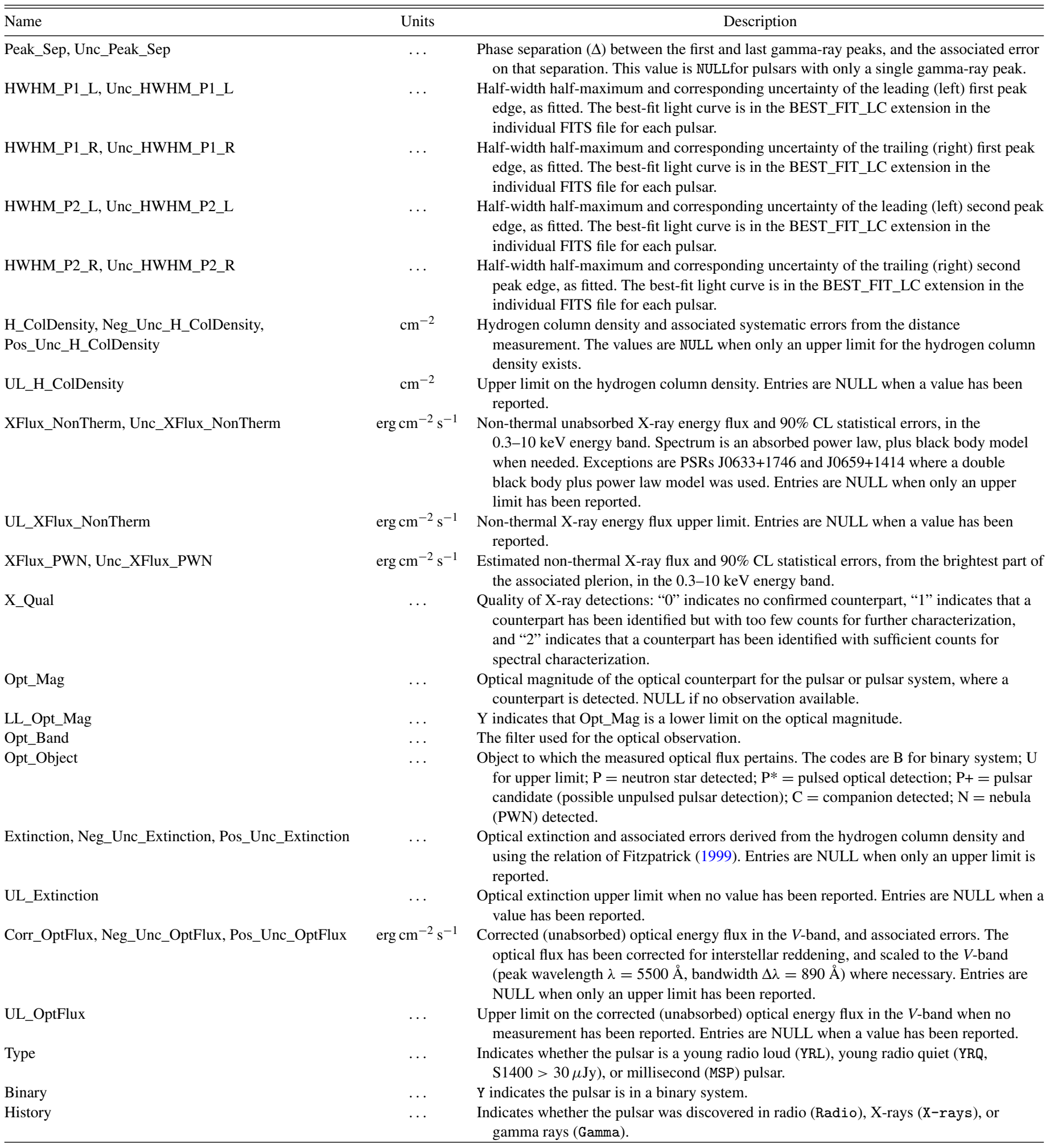

\section{B.2. Individual Pulsar FITS Files}

In addition to the summary information for each pulsar contained in the main catalog file, detailed results of the analyses are provided in the individual pulsar FITS files. Each file contains a variable number of FITS table extensions: PULSAR_SED, MODEL_SED, GAMMA_LC, BEST_FIT_LC, and RADIO_PROFILE (for radio detected pulsars). Again, the primary extension is empty. These files are also provided in ascii format with the content of the FITS tables appended in the order listed above in the file 2PC_auxiliary_files_v\#\#.tgz available at http://fermi.gsfc.nasa.gov/ssc/data/access/lat/2nd_PSR_ catalog/.

The PULSAR_SED extension (Table 22) contains the photon and energy fluxes for each pulsar in either six or twelve energy bins, fitting the pulsar with a power-law spectral form. These 
Table 19

LAT Second Pulsar Catalog FITS Format: SPECTRAL Extension

\begin{tabular}{|c|c|c|}
\hline Name & Units & Description \\
\hline PSR_Name & & Pulsar name. \\
\hline On_Peak & $\ldots$ & Y indicates the spectral fit used only on-peak events. \\
\hline TS_DC & $\cdots$ & $\begin{array}{l}\text { The test statistic obtained at the position of the pulsar, assuming a PLSuperExpCutoff spectral } \\
\text { model with the exponential index fixed to } 1 \text {. The fit uses data from } 100 \mathrm{MeV} \text { to } 100 \mathrm{GeV} \text {, and } \\
\text { includes all pulse phases. }\end{array}$ \\
\hline TS_Cutoff & $\cdots$ & $\begin{array}{l}\text { The significance of the spectral cutoff, obtained from the improvement in log(Likelihood) from } \\
\text { the PLSuperExpCut of } f \text { spectral model fit over the PowerLaw spectral fit. }\end{array}$ \\
\hline TS_bfree & $\cdots$ & $\begin{array}{l}\text { The improvement in the test statistic when the photon index is left free in the PLSuperExpCutoff } \\
\text { spectral fit. If there is no improvement, or the fit is worse, this value is zero. }\end{array}$ \\
\hline $\begin{array}{l}\text { PLEC1_Prefactor, } \\
\text { Unc_ECPL1_Prefactor }\end{array}$ & $\mathrm{ph} \mathrm{cm}^{-2} \mathrm{~s}^{-1} \mathrm{MeV}^{-1}$ & $\begin{array}{l}\text { The best-fit prefactor and associated error for the spectral fit using a power law with exponential } \\
\text { cutoff model where the exponential index is fixed at a value of } 1 .\end{array}$ \\
\hline $\begin{array}{l}\text { PLEC1_Photon_Index, } \\
\text { Unc_ECPL1_Photon_Index }\end{array}$ & $\ldots$ & $\begin{array}{l}\text { The best-fit photon index and associated error for the spectral fit using a PLSuperExpCutoff } \\
\text { model where the exponential index is fixed at a value of } 1 \text {. }\end{array}$ \\
\hline PLEC1_Scale & $\mathrm{MeV}$ & $\begin{array}{l}\text { The scaling energy for the spectral fit using a PLSuperExpCutoff model where the exponential } \\
\text { index is fixed at a value of } 1 .\end{array}$ \\
\hline PLEC1_Cutoff, Unc_PLEC1_Cutoff & $\mathrm{MeV}$ & $\begin{array}{l}\text { The best-fit cutoff energy and associated error for the spectral fit using a PLSuperExpCutoff } \\
\text { model where the exponential index is fixed at a value of } 1 \text {. }\end{array}$ \\
\hline PLEC1_Flux, Unc_PLEC1_Flux & $\mathrm{ph} \mathrm{cm}^{-2} \mathrm{~s}^{-1}$ & $\begin{array}{l}\text { The photon flux integrated from } 100 \mathrm{MeV} \text { to } 100 \mathrm{GeV} \text { and associated error for the spectral fit } \\
\text { using a PLSuperExpCut off model where the exponential index is fixed at a value of } 1 .\end{array}$ \\
\hline PLEC1_EFlux, Unc_PLEC1_EFlux & $\operatorname{erg~cm}{ }^{-2} \mathrm{~s}^{-1}$ & $\begin{array}{l}\text { The energy flux integrated from } 100 \mathrm{MeV} \text { to } 100 \mathrm{GeV} \text { and associated error for the spectral fit } \\
\text { using a PLSuperExpCutoff model where the exponential index is fixed at a value of } 1 \text {. }\end{array}$ \\
\hline $\begin{array}{l}\text { PLEC_Prefactor, } \\
\text { Unc_PLEC_Prefactor }\end{array}$ & $\mathrm{ph} \mathrm{cm}^{-2} \mathrm{~s}^{-1} \mathrm{MeV}^{-1}$ & $\begin{array}{l}\text { The best-fit prefactor and associated error for the spectral fit using a PLSuperExpCutoff model } \\
\text { where the exponential index is left free. }\end{array}$ \\
\hline $\begin{array}{l}\text { PLEC_Photon_Index, } \\
\text { Unc_PLEC_Photon_Index }\end{array}$ & $\cdots$ & $\begin{array}{l}\text { The best-fit photon index and associated error for the spectral fit using a PLSuperExpCutoff } \\
\text { model where the exponential index is left free. }\end{array}$ \\
\hline PLEC_Scale & $\mathrm{MeV}$ & $\begin{array}{l}\text { The scaling energy for the spectral fit using a PLSuperExpCut of fmodel where the exponential } \\
\text { index is left free. }\end{array}$ \\
\hline PLEC_Cutoff, Unc_PLEC_Cutoff & $\mathrm{MeV}$ & $\begin{array}{l}\text { The best-fit cutoff energy and associated error for the spectral fit using a PLSuperExpCutoff } \\
\text { model where the exponential index is left free. }\end{array}$ \\
\hline $\begin{array}{l}\text { PLEC_Exponential_Index, } \\
\text { Unc_PLEC_Exponential_Index }\end{array}$ & $\ldots$ & $\begin{array}{l}\text { The best-fit value and associated error for the spectral fit using a PLSuperExpCutoff model } \\
\text { where the exponential index is left free. }\end{array}$ \\
\hline PLEC_Flux, Unc_PLEC_Flux & $\mathrm{ph} \mathrm{cm}^{-2} \mathrm{~s}^{-1}$ & $\begin{array}{l}\text { The photon flux integrated from } 100 \mathrm{MeV} \text { to } 100 \mathrm{GeV} \text { and associated error for the spectral fit } \\
\text { using a PLSuperExpCut off model where the exponential index is left free. }\end{array}$ \\
\hline PLEC_EFlux, Unc_PLEC_EFlux & $\operatorname{erg~cm}{ }^{-2} \mathrm{~s}^{-1}$ & $\begin{array}{l}\text { The energy flux integrated from } 100 \mathrm{MeV} \text { to } 100 \mathrm{GeV} \text { and associated error for the spectral fit } \\
\text { using a PLSuperExpCut of } f \text { model where the exponential index is left free. }\end{array}$ \\
\hline PL_Prefactor, Unc_PL_Prefactor & $\mathrm{ph} \mathrm{cm}^{-2} \mathrm{~s}^{-1} \mathrm{MeV}^{-1}$ & The best-fit prefactor and associated error for the spectral fit using a PowerLaw model. \\
\hline $\begin{array}{l}\text { PL_Photon_Index, } \\
\text { Unc_PL_Photon_Index }\end{array}$ & $\cdots$ & The best-fit photon index and associated error for the spectral fit using a PowerLaw model. \\
\hline PL_Scale & $\mathrm{MeV}$ & The scaling energy for the spectral fit using a PowerLawmodel. \\
\hline PL_Flux, Unc_PL_Flux & $\mathrm{ph} \mathrm{cm}^{-2} \mathrm{~s}^{-1}$ & $\begin{array}{l}\text { The photon flux integrated from } 100 \mathrm{MeV} \text { to } 100 \mathrm{GeV} \text { and associated error for the spectral fit } \\
\text { using a PowerLaw model. }\end{array}$ \\
\hline PL_EFlux, Unc_PL_EFlux & $\operatorname{erg~cm}{ }^{-2} \mathrm{~s}^{-1}$ & $\begin{array}{l}\text { The energy flux integrated from } 100 \mathrm{MeV} \text { to } 100 \mathrm{GeV} \text { and associated error for the spectral fit } \\
\text { using a PowerLaw model. }\end{array}$ \\
\hline
\end{tabular}

points correspond to the black data points in the pulsar spectral energy distribution (SED) image files. The number of energy bins used in the SED varies with the significance of the pulsar. In a few cases, the pulsar is too faint to construct an SED or there were problems with the spectral fit, and this extension is not included.

The MODEL_SED extension (Table 23) contains the model photon flux and bowtie uncertainty using the PLEC1 spectral form fitted over the full energy range. A description of the spectral fitting method is provided in Section 6.1. These points correspond to the red curves in the pulsar SED image files. In cases where the pulsar is too faint to construct an SED, this extension is not reported.

The GAMMA_LC extension (Table 24) contains weighted counts and the corresponding uncertainties for light curves in six different energy ranges. The number of points in each light curve varies with the significance of the pulsar. These points correspond to the light curves shown in black in the pulsar light curve image files. The values for the background shown in those images are provided as keywords in the header of this FITS extension.

The BEST_FIT_LC extension (Table 25) reports the fitted light curve that best represents the data, as described in Section 5. These points correspond to the blue curves shown in the pulsar light curve image files.

The RADIO_PROFILE extension (Table 26) reports the radio profile for the radio loud pulsars. These points correspond to the red curves shown in the pulsar light curve image files. This extension is not included for pulsars undetected in radio.

\section{APPENDIX C}

\section{OFF-PEAK INDIVIDUAL SOURCE DISCUSSION}

Here we discuss several interesting sources found in the offpeak analysis presented in Section 7.

The off-peak emission from PSR J0007+7303 in the SNR CTA1 was previously studied by Abdo et al. (2012). They found a soft and not-significantly cut off source in the off-peak region 
Table 20

LAT Second Pulsar Catalog FITS Format: OFF_PEAK Extension

\begin{tabular}{|c|c|c|}
\hline Name & Units & Description \\
\hline PSR_Name & $\ldots$ & Pulsar name. \\
\hline Classification_OP & $\ldots$ & $\begin{array}{l}\text { Off-peak emission class: } M \text { for magnetospheric ("pulsar-like"), W for possible PWN emission, and } \\
\text { U for Unidentified. L is for sources with no significant off-peak emission. }\end{array}$ \\
\hline Min_Phase_OP, Max_Phase_OP & $\ldots$ & The minimum and maximum phase that defines the off-peak interval. \\
\hline $\begin{array}{l}\text { Min_2_Phase_OP, } \\
\text { Max_2_Phase_OP }\end{array}$ & $\cdots$ & $\begin{array}{l}\text { For pulsars with two off-peak phase ranges, the minimum and maximum phase for the second } \\
\text { off-peak interval. }\end{array}$ \\
\hline TS_point_OP & $\ldots$ & $\begin{array}{l}\text { The test statistic obtained at the best-fit position of the assumed point-like source. TS is computed } \\
\text { at the best-fit position assuming a power-law spectral model (except for PSR J0534+2200 as is } \\
\text { described in Section 7.2). }\end{array}$ \\
\hline TS_ext_OP & $\ldots$ & The significance of any possible extension for the source. \\
\hline TS_cutoff_OP & $\cdots$ & $\begin{array}{l}\text { The significance of any spectral cutoff for a source detected in the off-peak region. (Computed at } \\
\text { the pulsar's position) }\end{array}$ \\
\hline TS_var_OP & $\ldots$ & The significance of variability in the off-pulse emission. \\
\hline Spectral_Model_OP & $\cdots$ & $\begin{array}{l}\text { For regions with a significant detection, this is the best spectral model selected by our analysis } \\
\text { procedure described in Section 7.2. The possible spectral models are PowerLaw, } \\
\text { PLSuperExpCutoff, and FileFunction and are consistent with naming convention in } \\
\text { gtlike. }\end{array}$ \\
\hline Flux_OP, Unc_Flux_OP & $\mathrm{ph} \mathrm{cm}^{-2} \mathrm{~s}^{-1}$ & $\begin{array}{l}\text { The best-fit photon flux and estimated statistical error. The flux is integrated from } 100 \mathrm{MeV} \text { to } \\
316 \mathrm{GeV} \text {. }\end{array}$ \\
\hline EFlux_OP, Unc_EFlux_OP & 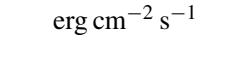 & $\begin{array}{l}\text { The best-fit energy flux and estimated statistical error. The flux is integrated from } 100 \mathrm{MeV} \text { to } \\
316 \mathrm{GeV} \text {. }\end{array}$ \\
\hline Prefactor_OP,Unc_Prefactor_OP & $\mathrm{ph} \mathrm{cm}^{-2} \mathrm{~s}^{-1} \mathrm{MeV}^{-1}$ & $\begin{array}{l}\text { The best-fit prefactor and estimated statistical error for the PowerLaw and PLSuperExpCutoff } \\
\text { spectral models. The prefactor is defined in Equations (B3) and (B4) for the two spectral } \\
\text { models. }\end{array}$ \\
\hline $\begin{array}{l}\text { Normalization_OP, } \\
\text { Unc_Normalization_OP }\end{array}$ & $\cdots$ & $\begin{array}{l}\text { The best-fit normalization and estimated statistical error for FileFunction spectral models. The } \\
\text { normalization is defined in Equation (B5). This spectral model was only used for the Crab } \\
\text { Nebula and Vela-X. }\end{array}$ \\
\hline Scale_OP & $\mathrm{MeV}$ & $\begin{array}{l}\text { The scaling energy for the PowerLaw and PLSuperExpCutoff spectral models. The scale is } \\
\text { defined in Equations (B3) and (B4) for the two spectral models. }\end{array}$ \\
\hline Index_OP, Unc_Index_OP & $\ldots$ & $\begin{array}{l}\text { The best-fit photon index and estimated statistical error for the PowerLaw and } \\
\text { PLSuperExpCutoff spectral models. The photon index is defined in Equations (B3) and (B4) } \\
\text { for the two spectral models. }\end{array}$ \\
\hline $\begin{array}{l}\text { Energy_Cutoff_OP, } \\
\text { Unc_Energy_Cutoff_OP }\end{array}$ & $\mathrm{MeV}$ & $\begin{array}{l}\text { The best-fit cutoff energy and the estimated statistical error for the PLSuperExpCutoff } \\
\text { spectral model. It is defined in Equation (B4). }\end{array}$ \\
\hline Spatial_Model_OP & $\cdots$ & $\begin{array}{l}\text { For off-peak regions with a significant detection, the spatial model selected by our analysis } \\
\text { procedure described in Section 7.2. The choices are At_Pulsar, Point, and Extended. }\end{array}$ \\
\hline RAJ2000_OP, DEJ2000_OP & $\operatorname{deg}$ & $\begin{array}{l}\text { The position of the source in celestial coordinates. For upper limits and sources with a best-fit } \\
\text { spatial model at the pulsar position, this is the pulsar's position. For sources where the } \\
\text { localized position is the selected spatial model, this is the best-fit position. For spatially } \\
\text { extended sources, this is the center of the best-fit extended source spatial model. }\end{array}$ \\
\hline GLON_OP, GLAT_OP & deg & This is the same as RA_J2000 and DEC_J2000, but in Galactic coordinates. \\
\hline Unc_Position_OP & $\operatorname{deg}$ & $\begin{array}{l}\text { For sources with a Point spatial model, the estimated statistical error on the localization of the } \\
\text { source. For sources with an Extended spatial model, the estimated statistical error on the } \\
\text { center of the extended source. }\end{array}$ \\
\hline Extension_OP, Unc_Extension_OP & deg & For sources with an Extended spatial model, the best fit extension and estimated statistical error. \\
\hline PowerLaw_Flux_UL_OP & $\mathrm{ph} \mathrm{cm}^{-2} \mathrm{~s}^{-1}$ & $\begin{array}{l}\text { For regions with no significant detection, this is the } 95 \% \text { confidence-level photon flux upper limit } \\
\text { computed assuming a PowerLaw spectral model with Index }=2 \text { and integrated from } \\
100 \mathrm{MeV} \text { to } 316 \mathrm{GeV} \text {. }\end{array}$ \\
\hline PowerLaw_EFlux_UL_OP & $\operatorname{erg~} \mathrm{cm}^{-2} \mathrm{~s}^{-1}$ & $\begin{array}{l}\text { The same as PowerLaw_Flux_UL, but instead the energy flux integrated from } 100 \mathrm{MeV} \text { to } \\
316 \mathrm{GeV} \text {. }\end{array}$ \\
\hline Cutoff_Flux_UL_OP & $\mathrm{ph} \mathrm{cm}^{-2} \mathrm{~s}^{-1}$ & $\begin{array}{l}\text { For regions with no significant detection, the } 95 \% \text { confidence-level photon flux upper limit } \\
\text { assuming a PLSuperExpCutof } f \text { spectral model with a canonical pulsar spectrum of } \\
\text { Index }=1.7 \text { and Energy_Cutoff }=3 \text {. This is the flux upper limit integrated from } 100 \mathrm{MeV} \\
\text { to } 316 \mathrm{GeV} \text {. }\end{array}$ \\
\hline Cutoff_EFlux_UL_OP & 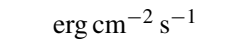 & The same as Cutoff_Flux_UL, but instead the energy flux integrated from $100 \mathrm{MeV}$ to $316 \mathrm{GeV}$. \\
\hline $\begin{array}{l}\text { SED_Lower_Energy_OP, } \\
\text { SED_Upper_Energy_OP, } \\
\text { SED_Center_Energy_OP }\end{array}$ & $\mathrm{MeV}$ & $\begin{array}{l}\text { For each region, we computed a spectral energy distribution (SED) for the source in } 14 \text { energy } \\
\text { bins spaced uniformly from } 100 \mathrm{MeV} \text { to } 316 \mathrm{GeV} \text { (4 bins per energy decade). Therefore, } \\
\text { each SED_* column corresponds to a vector of } 14 \text { values, one for each energy bin. } \\
\text { SED_Lower_Energy, SED_Upper_Energy, and SED_Middle_Energy are the lower } \\
\text { energy, upper energy, and energy in the geometric mean of the energy bin for each SED point. }\end{array}$ \\
\hline SED_TS_OP & & The test statistic obtained for each SED point. \\
\hline $\begin{array}{l}\text { SED_Prefactor_OP, } \\
\text { Neg_Unc_SED_Prefactor_OP, } \\
\text { Pos_Unc_SED_Prefactor_OP, } \\
\text { SED_Prefactor_UL_OP }\end{array}$ & $\mathrm{ph} \mathrm{cm}^{-2} \mathrm{~s}^{-1} \mathrm{MeV}^{-1}$ & $\begin{array}{l}\text { The best-fit prefactor, asymmetric lower and upper error, and } 95 \% \text { confidence-level upper limit } \\
\text { computed for the source in each energy bin. When TS } \geqslant 25 \text {, a detection is quoted when } \\
\text { SED_TS }>4 \text { and an upper limit is quoted otherwise. When TS }<25 \text {, all SED points are } \\
\text { quoted as upper limits. }\end{array}$ \\
\hline
\end{tabular}


Table 21

LAT Second Pulsar Catalog FITS Format: REFERENCES Extension

\begin{tabular}{|c|c|c|}
\hline Name & Units & Description \\
\hline Ref_Number & $\ldots$ & Numerical value of the reference from Distance_Ref and S1400_Ref columns. \\
\hline Citation & $\ldots$ & Citation for each reference. \\
\hline ADS_URL & $\ldots$ & $\begin{array}{l}\text { URL for the reference at the Astrophysical Data Service (ADS). This webpage provides links to the } \\
\text { original publishing journal of the referenced paper, article, or catalog. }\end{array}$ \\
\hline Title & . & Title of the reference. \\
\hline
\end{tabular}

Table 22

LAT Second Individual Pulsar FITS File Format: PULSAR_SED Extension

\begin{tabular}{|c|c|c|}
\hline Name & Units & Description \\
\hline Energy_Min, Energy_Max & $\mathrm{GeV}$ & Lower and upper bounds for each SED bin. \\
\hline Center_Energy & $\mathrm{GeV}$ & Central energy for each SED bin. \\
\hline PhotonFlux & $\mathrm{ph} \mathrm{cm}^{-2} \mathrm{~s}^{-1}$ & Photon flux in bin \\
\hline Unc_PhotonFlux & $\mathrm{ph} \mathrm{cm}^{-2} \mathrm{~s}^{-1}$ & $\begin{array}{l}\text { Best-fit value and associated error for the photon flux in each SED bin using a power law spectral model. } \\
\text { The error is set to zero when the given photon flux is an upper limit. }\end{array}$ \\
\hline EnergyFlux, Unc_EnergyFlux & $\mathrm{erg} \mathrm{cm}^{-2} \mathrm{~s}^{-1}$ & $\begin{array}{l}\text { Best-fit value and associated error for the energy flux in each SED bin using a power law spectral model. } \\
\text { The error is set to zero when the given energy flux is an upper limit. }\end{array}$ \\
\hline
\end{tabular}

Table 23

LAT Second Individual Pulsar FITS File Format: MODEL_SED Extension

\begin{tabular}{lcl}
\hline \hline Name & Units & Description \\
\hline $\begin{array}{l}\text { Energy_Min, Energy_Max } \\
\text { Center_Energy }\end{array}$ & $\mathrm{GeV}$ & $\begin{array}{l}\text { Lower and upper bounds for each SED bin. } \\
\text { Model_PhotonFlux }\end{array}$ \\
$\begin{array}{c}\text { Central energy for each SED bin. } \\
\text { Bowtie_Flux }\end{array}$ & $\mathrm{ph} \mathrm{cm}^{-2} \mathrm{~s}^{-1}$ & $\begin{array}{c}\text { Integrated photon flux in each bin calculated from the PLSuperExpCutoff model with the exponential index fixed } \\
\text { at a value of 1 that has been fitted over the full energy range (from 100 MeV to 100 GeV). } \\
\text { One-sigma uncertainty on the Model_PhotonFlux used to construct the bowtie on the spectral plots. }\end{array}$
\end{tabular}

Table 24

LAT Second Individual Pulsar FITS File Format: GAMMA_LC Extension

\begin{tabular}{|c|c|c|}
\hline Name & Units & Description \\
\hline Phase_Min, Phase_Max & & Lower and upper bounds for each bin in the gamma-ray light curve. \\
\hline $\begin{array}{l}\text { GT100_WtCounts, } \\
\text { Unc_GT100 WtCounts }\end{array}$ & $\ldots$ & $\begin{array}{l}\text { Weighted counts and associated error in each phase bin for the gamma-ray light curve } \\
\text { using an energy range of } 100 \mathrm{MeV} \text { to } 100 \mathrm{GeV} \text {. }\end{array}$ \\
\hline $\begin{array}{l}\text { GT3000_WtCounts, } \\
\text { Unc_GT3000_WtCounts }\end{array}$ & $\ldots$ & $\begin{array}{l}\text { Weighted counts and associated error in each phase bin for the gamma-ray light curve } \\
\text { using an energy range of } 3 \mathrm{GeV} \text { to } 100 \mathrm{GeV} \text {. }\end{array}$ \\
\hline $\begin{array}{l}\text { GT10000_WtCounts, } \\
\text { Unc_GT10000_WtCounts }\end{array}$ & $\ldots$ & $\begin{array}{l}\text { Weighted counts and associated error in each phase bin for the gamma-ray light curve } \\
\text { using an energy range of } 10 \mathrm{GeV} \text { to } 100 \mathrm{GeV} \text {. }\end{array}$ \\
\hline $\begin{array}{l}\text { 100_300_WtCounts, } \\
\text { Unc_100_300_WtCounts }\end{array}$ & $\ldots$ & $\begin{array}{l}\text { Weighted counts and associated error in each phase bin for the gamma-ray light curve } \\
\text { using an energy range of } 100 \mathrm{MeV} \text { to } 300 \mathrm{MeV} \text {. }\end{array}$ \\
\hline $\begin{array}{l}\text { 300_1000_WtCounts, } \\
\text { Unc_300_1000_WtCounts }\end{array}$ & $\ldots$ & $\begin{array}{l}\text { Weighted counts and associated error in each phase bin for the gamma-ray light curve } \\
\text { using an energy range of } 300 \mathrm{MeV} \text { to } 1 \mathrm{GeV} \text {. }\end{array}$ \\
\hline $\begin{array}{l}\text { 1000_3000_WtCounts, } \\
\text { Unc_1000_3000_WtCounts }\end{array}$ & $\ldots$ & $\begin{array}{l}\text { Weighted counts and associated error in each phase bin for the gamma-ray light curve } \\
\text { using an energy range of } 1 \text { to } 3 \mathrm{GeV} \text {. }\end{array}$ \\
\hline
\end{tabular}

Table 25

LAT Second Individual Pulsar FITS File Format: BEST_FIT_LC Extension

\begin{tabular}{lcc}
\hline \hline Name & Units & \\
\hline Phase_Min, Phase_Max & $\ldots$ & Lower and upper bounds for each bin in the best fit gamma-ray light curve. \\
Norm_Intensity & $\ldots$ & $\begin{array}{c}\text { Normalized gamma-ray intensity for each bin in the best fit gamma-ray light curve. The intensity is normalized so that the } \\
\text { integral of the profile is } \sim 1 \text { (i.e., normalized as a density function). }\end{array}$ \\
\hline
\end{tabular}

Table 26

LAT Second Individual Pulsar FITS File Format: RADIO_PROFILE Extension

\begin{tabular}{|c|c|c|}
\hline Name & Units & Description \\
\hline Phase_Min, Phase_Max & $\ldots$ & Lower and upper bounds for each bin in the radio light curve. \\
\hline Norm_Intensity & $\ldots$ & Normalized radio flux for each bin in the radio light curve. The flux is normalized so that the peak flux equals 1 . \\
\hline
\end{tabular}


that is marginally extended. We find a similar spectrum and extension significance $\left(\mathrm{TS}_{\mathrm{ext}}=10.8\right)$, and therefore classify this source as type "U."

The new type "W" source is associated with PSR J0205+6449 (Abdo et al. 2009c). The off-peak spectrum for this source is shown in panel (b) of Figure 13. The emission is best fit as a point source at $(l, b)=(130.73,3.11)$ with a $95 \%$ confidencelevel radius of 0.03 . The source has a hard spectrum (power law with $\Gamma=1.61 \pm 0.21$ ) and is therefore consistent with a PWN hypothesis. This nebula has been observed at infrared (Slane et al. 2008) and X-ray (Slane et al. 2004) energies. This suggests that we could be observing the inverse Compton emission from the same electrons powering synchrotron emission at lower energies. The PWN hypothesis is supported by the associated pulsar's very high $\dot{E}=2.6 \times 10^{36} \mathrm{erg} \mathrm{s}^{-1}$ and relatively young characteristic age, $\tau_{c}=5400 \mathrm{yr}$. This is consistent with the properties of other pulsars with LAT-detected PWN, and we favor a PWN interpretation. We note that the discrepancy between our spectrum and the upper limit quoted in Ackermann et al. (2011) is mainly caused by our expanded energy range and because the flux upper limit was computed assuming a different spectral index.

However, we note that PSR J0205+6449 is associated to the SNR 3C58 $(\mathrm{G} 130.7+3.1)$. Given the $2 \mathrm{kpc}$ distance estimate from Section 4.2 and the density of thermal material estimated by Slane et al. (2004), we can estimate the energetics required for the LAT emission to originate in the SNR. Following the prescription in Drury et al. (1994), we assume the LAT emission to be hadronic and estimate a cosmic-ray efficiency for the SNR of $\sim 10 \%$, which is energetically allowed. We therefore cannot rule out the SNR hypothesis.

No $\mathrm{TeV}$ detection of this source has been reported, but given the hard photon index at $\mathrm{GeV}$ energies this is a good candidate for observations by an atmospheric Cherenkov telescope. Improved spectral and spatial observations at $\mathrm{TeV}$ energies might help to uniquely classify the emission.

We obtain a flux for Vela-X which is $\sim 10 \%$ larger than the flux obtained in Grondin et al. (2013). This discrepancy is mostlikely due to assuming a different spatial model for the emission (radially-symmetric Gaussian compared to elliptical Gaussian).

PSR J1023-5746 is associated with the TeV PWN HESS J1023-575 (Aharonian et al. 2007). LAT emission from this PWN was first reported in Ackermann et al. (2011). Because of the dominant low-energy magnetospheric emission, we classify this as type "M" and not as a PWN. A phase-averaged analysis of this source for energies above $10 \mathrm{GeV}$ is reported in Acero et al. (2013a).

PSR J1119-6127 (Parent et al. 2011) is associated with the TeV source HESS J1119-614. ${ }^{102}$ Our off-peak analysis classifies this source as "U" because its spectrum is soft and not significantly cut off. However, the SED appears to represent a cutoff spectrum at low energy and a hard rising spectrum at high energy. Acero et al. (2013a) significantly detect this PWN using the analysis procedure as described for J1023-575. We are likely detecting a composite of magnetospheric emission at low energy and pulsar-wind emission at high energy.

PSR J1357-6429 (Lemoine-Goumard et al. 2011) has an associated PWN HESS J1356-645 detected at TeV energies (Abramowski et al. 2011). Our analysis of the off-peak regions surrounding PSR J1357-6429 shows a source positionally

\footnotetext{
102 The discovery of HESS J1119-614 was presented at the "Supernova Remnants and Pulsar Wind Nebulae in the Chandra Era" in 2009. See http://cxc.harvard.edu/cdo/snr09/pres/DjannatiAtai_Arache_v2.pdf.
}

and spectrally consistent with HESS J1356-645, but with significance just below detection threshold $(\mathrm{TS}=21.0)$. Acero et al. (2013a) present significant emission from this source.

The off-peak region of PSR J1410-6132 (O'Brien et al. 2008) shows a relatively hard spectral index of $1.90 \pm 0.15$, and the spectrum is not significantly cut off. There is no associated $\mathrm{TeV}$ PWN and enough low-energy GeV emission is present to caution against a clear PWN interpretation. We classify this source as "U," but further observations could reveal interesting emission.

PSR J2021+4026 is spatially coincident with the LATdetected and spatially extended Gamma Cygni SNR (Lande et al. 2012). The off-peak emission from this pulsar is consistent with an exponentially cutoff spectrum and is therefore classified as type "M." The source's marginal extension $\left(\mathrm{TS}_{\mathrm{ext}}=8.7\right)$ is likely due to some contamination from the SNR.

\section{REFERENCES}

Abdo, A. A., Ackermann, M., Ajello, M., et al. 2009a, ApJL, 706, L1 (W51C) Abdo, A. A., Ackermann, M., Ajello, M., et al. 2010a, A\&A, 524, A75 (Globular Clusters)

Abdo, A. A., Ackermann, M., Ajello, M., et al. 2010b, ApJ, 714, 927 (PSR B1509-58)

Abdo, A. A., Ackermann, M., Ajello, M., et al. 2010c, ApJ, 708, 1254 (Crab pulsar and PWN)

Abdo, A. A., Ackermann, M., Ajello, M., et al. 2010d, ApJ, 718, 348 (W28)

Abdo, A. A., Ackermann, M., Ajello, M., et al. 2010e, ApJ, 713, 146 (Vela-X PWN)

Abdo, A. A., Ackermann, M., Ajello, M., et al. 2010f, ApJL, 710, L92 (Cassiopeia A)

Abdo, A. A., Ackermann, M., Ajello, M., et al. 2010g, ApJ, 720, 272 (Geminga) Abdo, A. A., Ackermann, M., Ajello, M., et al. 2010h, ApJ, 722, 1303 (W49B) Abdo, A. A., Ackermann, M., Ajello, M., et al. 2010i, Sci, 327, 1103 (W44)

Abdo, A. A., Ackermann, M., Ajello, M., et al. 2010j, ApJ, 712, 459 (IC 443) Abdo, A. A., Ackermann, M., Ajello, M., et al. 2010k, A\&A, 512, A7 (LMC)

Abdo, A. A., Ackermann, M., Ajello, M., et al. 20101, ApJ, 711, 64 (PSR J1907+0602)

Abdo, A. A., Ackermann, M., Ajello, M., et al. 2010m, ApJS, 187, 460 (1PC)

Abdo, A. A., Ackermann, M., Ajello, M., et al. 2010n, ApJ, 713, 154 (Vela Pulsar 11 months)

Abdo, A. A., Ackermann, M., Ajello, M., et al. 2011a, Sci, 331, 739 (Crab Flare)

Abdo, A. A., Ackermann, M., Ajello, M., et al. 2011b, ApJ, 734, 28 (RX J1713.7-3946)

Abdo, A. A., Ackermann, M., Atwood, W. B., et al. 2009b, Sci, 325, 840 (Blind Search Pulsars)

Abdo, A. A., Ackermann, M., Atwood, W. B., et al. 2009c, ApJL, 699, L102 (PSR J0205+6449)

Abdo, A. A., Ackermann, M., Atwood, W. B., et al. 2009d, ApJ, 700, 1059 (PSR J2021+3651)

Abdo, A. A., Ackermann, M., Atwood, W. B., et al. 2009e, ApJ, 699, 1171 (PSR J0030+0451)

Abdo, A. A., Ackermann, M., Atwood, W. B., et al. 2009f, APh, 32, 193 (On-orbit calibration)

Abdo, A. A., Ajello, M., Antolini, E., et al. 2010o, ApJ, 720, 26 (PSRs J1057-5226, J1709-4429, and J1952+3252)

Abdo, A. A., Wood, K. S., DeCesar, M. E., et al. 2012, ApJ, 744, 146 (PSR J0007+7303)

Abramowski, A., Acero, F., Aharonian, F., et al. 2011, A\&A, 533, A103

Acero, F., Ackermann, M., Ajello, M., et al. 2013a, ApJ, 773, 77

Acero, F., Gallant, Y., Ballet, J., Renaud, M., \& Terrier, R. 2013b, A\&A, 551, A7

Ackermann, M., Ajello, M., Albert, A., et al. 2012a, ApJS, 203, 4 (Pass 7 Performance)

Ackermann, M., Ajello, M., Allafort, A., et al. 2012b, ApJ, 753, 83 (Unassociated 1FGL Sources)

Ackermann, M., Ajello, M., Baldini, L., et al. 2011, ApJ, 726, 35 (LAT PWN catalog)

Aharonian, F., Akhperjanian, A. G., Bazer-Bachi, A. R., et al. 2007, A\&A, 467, 1075

Ajello, M., Allafort, A., Baldini, L., et al. 2012, ApJ, 744, 80

Aleksić, J., Alvarez, E. A., Antonelli, L. A., et al. 2012, A\&A, 540, A69

Aliu, E., Anderhub, H., Antonelli, L. A., et al. 2008, Sci, 322, 1221 
Aliu, E., Arlen, T., Aune, T., et al. 2011, Sci, 334, 69

Alpar, M. A., Cheng, A. F., Ruderman, M. A., \& Shaham, J. 1982, Natur, 300,728

Arons, J. 1996, A\&AS, 120, C49

Arumugasamy, P., Pavlov, G., \& Kargaltsev, O. 2013, in XMM-Newton 2013 Science Workshop: The Fast and the Furious: Energetic Phenomena in Isolated Neutron Stars, Pulsar Wind Nebulae, and Supernova Remnants, ed. J.-U. Ness (Madrid: ESAC), 49

Arzoumanian, Z., Fruchter, A. S., \& Taylor, J. H. 1994, ApJL, 426, L85

Arzoumanian, Z., Gotthelf, E. V., Ransom, S. M., et al. 2011, ApJ, 739, 39

Atwood, W. B., Abdo, A. A., Ackermann, M., et al. 2009, ApJ, 697, 1071 (LAT Instrument Paper)

Atwood, W. B., Ziegler, M., Johnson, R. P., \& Baughman, B. M. 2006, ApJL, 652, L49

Bai, X.-N., \& Spitkovsky, A. 2010, ApJ, 715, 1282

Bailes, M., Harrison, P. A., Lorimer, D. R., et al. 1994, ApJL, 425, L41

Barr, E. D., Guillemot, L., Champion, D. J., et al. 2013, MNRAS, 429, 1633

Bassa, C. G., van Kerkwijk, M. H., \& Kulkarni, S. R. 2003, A\&A, 403, 1067

Bassa, C. G., van Kerkwijk, M. H., \& Kulkarni, S. R. 2006, A\&A, 450, 295

Bates, S. D., Bailes, M., Bhat, N. D. R., et al. 2011, MNRAS, 416, 2455

Becker, W., \& Truemper, J. 1997, A\&A, 326, 682

Bhalerao, V. B., \& Kulkarni, S. R. 2011, ApJL, 737, L1

Bhattacharyya, B., Roy, J., Ray, P. S., et al. 2013, ApJL, 773, L12

Bignami, G. F., \& Caraveo, P. A. 1996, ARA\&A, 34, 331

Bignami, G. F., Caraveo, P. A., \& Mereghetti, S. 1993, Natur, 361, 704

Boyles, J., Lorimer, D. R., McLaughlin, M. A., et al. 2011, in AIP Conf. Proc. 1357, Radio Pulsars: An Astrophysical Key to Unlock the Secrets of the Universe, ed. M. Burgay et al. (Melville, NY: AIP), 32

Breton, R. P., van Kerkwijk, M. H., Roberts, M. S. E., et al. 2013, ApJ, 769, 108

Buehler, R., Scargle, J. D., Blandford, R. D., et al. 2012, ApJ, 749, 26

Burgay, M., Joshi, B. C., D’Amico, N., et al. 2006, MNRAS, 368, 283

Burrows, D. N., Hill, J. E., Nousek, J. A., et al. 2005, SSRv, 120, 165

Butler, R. F., Golden, A., \& Shearer, A. 2002, A\&A, 395, 845

Camilo, F., Kerr, M., Ray, P. S., et al. 2012, ApJ, 746, 39

Camilo, F., Ransom, S. M., Gaensler, B. M., et al. 2006, ApJ, 637, 456

Camilo, F., Ray, P. S., Ransom, S. M., et al. 2009, ApJ, 705, 1

Caraveo, P. A., Bignami, G. F., \& Mereghetti, S. 1994, ApJL, 422, L87

Carlberg, R. G., \& Innanen, K. A. 1987, AJ, 94, 666

Caswell, J. L., McClure-Griffiths, N. M., \& Cheung, M. C. M. 2004, MNRAS, 352,1405

Celik, O., \& Johnson, T. J. 2011, in AIP Conf. Proc. 1357, Radio Pulsars: An Astrophysical Key to Unlock the Secrets of the Universe, ed. M. Burgay et al. (Melville, NY: AIP), 225

Chen, K. 1991, Natur, 352, 695

Cheng, K. S., Ho, C., \& Ruderman, M. 1986, ApJ, 300, 500

Cocke, W. J., Disney, M. J., \& Taylor, D. J. 1969, Natur, 221, 525

Cognard, I., Bourgois, G., Lestrade, J.-F., et al. 1995, A\&A, 296, 169

Cognard, I., Guillemot, L., Johnson, T. J., et al. 2011, ApJ, 732, 47

Collins, S., Shearer, A., \& Mignani, R. 2011, in AIP Conf. Proc. 1357, Radio Pulsars: An Astrophysical Key to Unlock the Secrets of the Universe, ed. M. Burgay et al. (Melville, NY: AIP), 310

Cordes, J. M., \& Lazio, T. J. W. 2002, arXiv:astro-ph/0207156

D’Amico, N., Stappers, B. W., Bailes, M., et al. 1998, MNRAS, 297, 28

Danilenko, A., Kirichenko, A., Mennickent, R. E., et al. 2012, A\&A, 540, A28 de Jager, O. C., \& Büsching, I. 2010, A\&A, 517, L9

de Jager, O. C., Raubenheimer, B. C., \& Swanepoel, J. W. H. 1989, A\&A, 221,180

De Luca, A., Caraveo, P. A., Mereghetti, S., Negroni, M., \& Bignami, G. F. 2005, ApJ, 623, 1051

De Luca, A., Marelli, M., Mignani, R. P., et al. 2011, ApJ, 733, 104

Deller, A. T., Brisken, W. F., Chatterjee, S., et al. 2011, in Proc. of the 20th EVGA Meeting, ed. W. Alef, S. Bernhart, \& A. Nothnagel (Bonn: MaxPlanck-Institut für Radioastronomie), 178

Deller, A. T., Tingay, S. J., Bailes, M., \& Reynolds, J. E. 2009, ApJ, 701, 1243

Demorest, P. B., Pennucci, T., Ransom, S. M., Roberts, M. S. E., \& Hessels, J. W. T. 2010, Natur, 467, 1081

Dodson, R., Legge, D., Reynolds, J. E., \& McCulloch, P. M. 2003, ApJ, 596, 1137

Dormody, M., Johnson, R. P., Atwood, W. B., et al. 2011, ApJ, 742, 126

Doroshenko, O., Löhmer, O., Kramer, M., et al. 2001, A\&A, 379, 579

Drury, L. O., Aharonian, F. A., \& Voelk, H. J. 1994, A\&A, 287, 959

Du, Y. J., Han, J. L., Qiao, G. J., \& Chou, C. K. 2011, ApJ, 731, 2

Dubus, G. 2006, A\&A, 456, 801

Dyks, J., Harding, A. K., \& Rudak, B. 2004, ApJ, 606, 1125

Dyks, J., \& Rudak, B. 2003, ApJ, 598, 1201

Ebisawa, K., Tsujimoto, M., Paizis, A., et al. 2005, ApJ, 635, 214

Espinoza, C. M., Guillemot, L., Çelik, Ö., et al. 2013, MNRAS, 430, 571
Fichtel, C. E., Hartman, R. C., Kniffen, D. A., et al. 1975, ApJ, 198, 163

Fitzpatrick, E. L. 1999, PASP, 111, 63

Freire, P. C. C., Abdo, A. A., Ajello, M., et al. 2011, Sci, 334, 1107

Gaensler, B. M., van der Swaluw, E., Camilo, F., et al. 2004, ApJ, 616, 383

Garmire, G. P., Bautz, M. W., Ford, P. G., Nousek, J. A., \& Ricker, G. R., Jr. 2003, Proc. SPIE, 4851, 28

Giordano, F., Naumann-Godo, M., Ballet, J., et al. 2012, ApJL, 744, L2

Gonthier, P. L., Story, S. A., Clow, B. D., \& Harding, A. K. 2007, Ap\&SS, 309,245

Gonzalez, M., \& Safi-Harb, S. 2003, ApJL, 583, L91

Greidanus, H., \& Strom, R. G. 1990, A\&A, 240, 376

Grondin, M.-H., Funk, S., Lemoine-Goumard, M., et al. 2011, ApJ, 738, 42

Grondin, M.-H., Romani, R. W., Lemoine-Goumard, M., et al. 2013, ApJ, in press

Guillemot, L., Freire, P. C. C., Cognard, I., et al. 2012, MNRAS, 422, 1294

Guillemot, L., Kramer, M., Johnson, T. J., et al. 2013, ApJ, 768, 169

Gupta, Y., Mitra, D., Green, D. A., \& Acharyya, A. 2005, CSci, 89, 853

Halpern, J. P., Camilo, F., Gotthelf, E. V., et al. 2001, ApJL, 552, L125

Halpern, J. P., Gotthelf, E. V., \& Camilo, F. 2012, ApJL, 753, L14

Halpern, J. P., Gotthelf, E. V., Mirabal, N., \& Camilo, F. 2002, ApJL, 573, L41

Hessels, J. W. T., Roberts, M. S. E., McLaughlin, M. A., et al. 2011, in AIP Conf. Proc. 1357, Radio Pulsars: An Astrophysical Key to Unlock the Secrets of the Universe, ed. M. Burgay et al. (Melville, NY: AIP), 40

Hessels, J. W. T., Roberts, M. S. E., Ransom, S. M., et al. 2004, ApJ, 612, 389

Hewitt, J. W., Grondin, M.-H., Lemoine-Goumard, M., et al. 2012, ApJ, 759,89

Hobbs, G., Edwards, R., \& Manchester, R. 2006, ChJAS, 6, 189

Hobbs, G., Faulkner, A., Stairs, I. H., et al. 2004a, MNRAS, 352, 1439

Hobbs, G., Lorimer, D. R., Lyne, A. G., \& Kramer, M. 2005, MNRAS, 360, 974

Hobbs, G. B., Lyne, A. G., Kramer, M., Martin, C. E., \& Jordan, C. 2004b, MNRAS, 353, 1311

Hotan, A. W., Bailes, M., \& Ord, S. M. 2006, MNRAS, 369, 1502

Hou, X., \& Smith, D. 2013, in XMM-Newton 2013 Science Workshop: The Fast and the Furious: Energetic Phenomena in Isolated Neutron Stars, Pulsar Wind Nebulae and Supernova Remnants, ed. J.-U. Ness (Madrid: ESAC), 56

Jackson, B., Sargle, J. D., Barnes, D., et al. 2005, ISPL, 12, 105

Janssen, G. H., \& Stappers, B. W. 2006, A\&A, 457, 611

Johnson, T. J., Guillemot, L., Kerr, M., et al. 2013, ApJ, submitted (B1821-24 in M28)

Johnston, S., Manchester, R. N., Lyne, A. G., Kaspi, V. M., \& D’Amico, N. 1995, A\&A, 293, 795

Kalapotharakos, C., Harding, A. K., Kazanas, D., \& Contopoulos, I. 2012a, ApJL, 754, L1

Kalapotharakos, C., Kazanas, D., Harding, A., \& Contopoulos, I. 2012b, ApJ, 749, 2

Kalberla, P. M. W., Burton, W. B., Hartmann, D., et al. 2005, A\&A, 440, 775

Kaplan, D. L., Stovall, K., Ransom, S. M., et al. 2012, ApJ, 753, 174

Karastergiou, A., \& Johnston, S. 2007, MNRAS, 380, 1678

Kargaltsev, O., \& Pavlov, G. G. 2008, in AIP Conf. Proc. 983, 40 Years of Pulsars: Millisecond Pulsars, Magnetars and More, ed. C. Bassa et al. (Melville, NY: AIP), 171

Kargaltsev, O., Pavlov, G. G., \& Romani, R. W. 2004, ApJ, 602, 327

Kaspi, V. M., Bailes, M., Manchester, R. N., et al. 1997, ApJ, 485, 820

Katagiri, H., Tibaldo, L., Ballet, J., et al. 2011, ApJ, 741, 44

Katsuda, S., Tsunemi, H., Mori, K., et al. 2012, ApJL, 754, L7

Katsuta, J., Uchiyama, Y., Tanaka, T., et al. 2012, ApJ, 752, 135

Keith, M. J. 2012, Proc. of IAUS 291 "Neutron Stars and Pulsars: Challenges and Opportunities after 80 Years,"arXiv: 1210.7868

Keith, M. J., Johnston, S., Bailes, M., et al. 2012, MNRAS, 419, 1752

Keith, M. J., Johnston, S., Kramer, M., et al. 2008, MNRAS, 389, 1881

Keith, M. J., Johnston, S., Ray, P. S., et al. 2011, MNRAS, 414, 1292

Kerr, M. 2011, ApJ, 732, 38

Kerr, M., Camilo, F., Johnson, T. J., et al. 2012, ApJL, 748, L2

King, A. R., Davies, M. B., \& Beer, M. E. 2003, MNRAS, 345, 678

Kong, A. K. H., Huang, R. H. H., Cheng, K. S., et al. 2012, ApJL, 747, L3

Kong, A. K. H., Hui, C. Y., \& Cheng, K. S. 2010, ApJL, 712, L36

Koptsevich, A. B., Lundqvist, P., Serafimovich, N. I., Shibanov, Y. A., \& Sollerman, J. 2003, A\&A, 400, 265

Kothes, R., Uyaniker, B., \& Pineault, S. 2001, ApJ, 560, 236

Kramer, M., Bell, J. F., Manchester, R. N., et al. 2003, MNRAS, 342, 1299

Kuijken, K., \& Gilmore, G. 1989, MNRAS, 239, 571

Kulkarni, S. R., Djorgovski, S., \& Fruchter, A. S. 1988, Natur, 334, 504

Kuulkers, E., den Hartog, P. R., in't Zand, J. J. M., et al. 2003, A\&A, 399, 663

Lande, J., Ackermann, M., Allafort, A., et al. 2012, ApJ, 756, 5

Landecker, T. L., Roger, R. S., \& Higgs, L. A. 1980, A\&AS, 39, 133

Lasker, B. M. 1976, ApJ, 203, 193

Lassus, A. 2013, PhD thesis, Université d'Orléans 
Lazaridis, K., Verbiest, J. P. W., Tauris, T. M., et al. 2011, MNRAS, 414, 3134

Lee, K. J., Guillemot, L., Yue, Y. L., Kramer, M., \& Champion, D. J. 2012, MNRAS, 424, 2832

Lemoine-Goumard, M., Zavlin, V. E., Grondin, M.-H., et al. 2011, A\&A, 533, A102

Li, J., Spitkovsky, A., \& Tchekhovskoy, A. 2012, ApJ, 746, 60

Li, W., Chornock, R., Leaman, J., et al. 2011, MNRAS, 412, 1473

Lorimer, D. R., Faulkner, A. J., Lyne, A. G., et al. 2006, MNRAS, 372, 777

Lorimer, D. R., \& Kramer, M. 2004, Handbook of Pulsar Astronomy (Vol. 4; Cambridge: Cambridge Univ. Press)

Lorimer, D. R., Yates, J. A., Lyne, A. G., \& Gould, D. M. 1995, MNRAS, 273,411

Lundgren, S. C., Foster, R. S., \& Camilo, F. 1996, in ASP Conf. Ser. 105, IAU Colloq. 160: Pulsars: Problems and Progress, ed. S. Johnston, M. A. Walker, \& M. Bailes (San Francisco, CA: ASP), 497

Lutz, T. E., \& Kelker, D. H. 1973, PASP, 85, 573

Lyne, A. G., Manchester, R. N., Lorimer, D. R., et al. 1998, MNRAS, 295,743

Lyutikov, M., Otte, N., \& McCann, A. 2012, ApJ, 754, 33

Maan, Y., Aswathappa, H. A., \& Deshpande, A. A. 2012, MNRAS, 425, 2

Malofeev, V. M., \& Malov, O. I. 1997, Natur, 389, 697

Manchester, R. N., Hobbs, G. B., Teoh, A., \& Hobbs, M. 2005, AJ, 129, 1993

Manchester, R. N., Lyne, A. G., Camilo, F., et al. 2001, MNRAS, 328, 17

Marelli, M. 2012, PhD thesis, Univ. Insubria, arXiv:1205.1748

Marelli, M., De Luca, A., \& Caraveo, P. A. 2011, ApJ, 733, 82

Maron, O., Kijak, J., Kramer, M., \& Wielebinski, R. 2000, A\&AS, 147, 195

Mattox, J. R., Bertsch, D. L., Chiang, J., et al. 1996, ApJ, 461, 396

Meegan, C., Lichti, G., Bhat, P. N., et al. 2009, ApJ, 702, 791

Mignani, R. P. 2011, AdSpR, 47, 1281

Mignani, R. P., \& Becker, W. 2004, AdSpR, 33, 616

Mignani, R. P., Caraveo, P. A., \& Bignami, G. F. 1999, A\&A, 343, L5

Mignani, R. P., Corongiu, A., Pallanca, C., \& Ferraro, F. R. 2013a, MNRAS, 430, 1008

Mignani, R. P., De Luca, A., Hummel, W., et al. 2012a, A\&A, 544, A100

Mignani, R. P., de Luca, A., Rea, N., et al. 2013b, MNRAS, 430, 1354

Mignani, R. P., Jackson, A. C., \& Spiers, A. 2010a, A\&A, 520, A21

Mignani, R. P., Pavlov, G. G., \& Kargaltsev, O. 2010b, ApJ, 720, 1635

Mignani, R. P., Perna, R., Rea, N., et al. 2007, A\&A, 471, 265

Mignani, R. P., Razzano, M., Esposito, P., et al. 2012b, A\&A, 543, A130

Mignani, R. P., Shearer, A., de Luca, A., et al. 2011, A\&A, 533, A101

Mitsuda, K., Bautz, M., Inoue, H., et al. 2007, PASJ, 59, 1

Moffett, D. A., \& Hankins, T. H. 1996, ApJ, 468, 779

Mori, K., Hailey, C. J., Paerels, F., \& Zane, S. 2004, AdSpR, 33, 503

Morris, D. J., Hobbs, G., Lyne, A. G., et al. 2002, MNRAS, 335, 275

Muslimov, A. G., \& Harding, A. K. 2004, ApJ, 606, 1143

Ng, C., \& HTRU Collaboration, 2013, in IAU Symp. 291, ed. J. van Leeuwen (Cambridge: Cambridge Univ. Press), 53

Ng, C.-Y., \& Romani, R. W. 2008, ApJ, 673, 411

Nice, D. J., Splaver, E. M., Stairs, I. H., et al. 2005, ApJ, 634, 1242

Nolan, P. L., Abdo, A. A., Ackermann, M., et al. 2012, ApJS, 199, 31

Novara, G., La Palombara, N., Carangelo, N., et al. 2006, A\&A, 448, 93

O'Brien, J. T., Johnston, S., Kramer, M., et al. 2008, MNRAS, 388, L1

Oka, T., Kawai, N., Naito, T., et al. 1999, ApJ, 526, 764

Pallanca, C., Mignani, R. P., Dalessandro, E., et al. 2012, ApJ, 755, 180

Parent, D., Kerr, M., den Hartog, P. R., et al. 2011, ApJ, 743, 170

Pellizzoni, A., Trois, A., Tavani, M., et al. 2010, Sci, 327, 663

Pétri, J. 2012, MNRAS, 424, 605

Pierbattista, M., Grenier, I. A., Harding, A. K., \& Gonthier, P. L. 2012, A\&A, 545, A 42

Pineault, S., Landecker, T. L., Madore, B., \& Gaumont-Guay, S. 1993, AJ, 105,1060

Pletsch, H. J., Guillemot, L., Allen, B., et al. 2012a, ApJ, 744, 105

Pletsch, H. J., Guillemot, L., Allen, B., et al. 2012b, ApJL, 755, L20

Pletsch, H. J., Guillemot, L., Fehrmann, H., et al. 2012c, Sci, 338, 1314

Predehl, P., \& Schmitt, J. H. M. M. 1995, A\&A, 293, 889

Radhakrishnan, V., \& Cooke, D. J. 1969, ApL, 3, 225

Ransom, S. M., Eikenberry, S. S., \& Middleditch, J. 2002, AJ, 124, 1788
Ransom, S. M., Ray, P. S., Camilo, F., et al. 2011, ApJL, 727, L16

Ravi, V., Manchester, R. N., \& Hobbs, G. 2010, ApJL, 716, L85

Ray, P. S., Abdo, A. A., Parent, D., et al. 2012, arXiv:1205.3089

Ray, P. S., Kerr, M., Parent, D., et al. 2011, ApJS, 194, 17

Ray, P. S., Ransom, S. M., Cheung, C. C., et al. 2013, ApJL, 763, L13

Ray, P. S., Thorsett, S. E., Jenet, F. A., et al. 1996, ApJ, 470, 1103

Razzano, M., Mignani, R. P., Marelli, M., \& de Luca, A. 2013, MNRAS, 428, 3636

Reichardt, I., de Oña-Wilhelmi, E., Rico, J., \& Yang, R. 2012, A\&A, 546, A21

Reid, M. J., Menten, K. M., Zheng, X. W., et al. 2009, ApJ, 700, 137

Roberts, M. S. E. 2013, in IAU Symp. 291, ed. J. van Leeuwen (Cambridge: Cambridge Univ. Press), 127

Romani, R. W. 2012, ApJL, 754, L25

Romani, R. W., Kerr, M., Craig, H. A., et al. 2011, ApJ, 738, 114

Romani, R. W., \& Shaw, M. S. 2011, ApJL, 743, L26

Romani, R. W., \& Watters, K. P. 2010, ApJ, 714, 810

Romani, R. W., \& Yadigaroglu, I.-A. 1995, ApJ, 438, 314

Ruiz, M. T., \& May, J. 1986, ApJ, 309, 667

Saz Parkinson, P. M., Dormody, M., Ziegler, M., et al. 2010, ApJ, 725, 571

Scargle, J. D., Norris, J. P., Jackson, B., \& Chiang, J. 2013, ApJ, 764, 167

Shearer, A., Mignani, R. P., Collins, S., et al. 2013, MNRAS, submitted

Shklovskii, I. S. 1970, SvA, 13, 562

Slane, P., Helfand, D. J., Reynolds, S. P., et al. 2008, ApJL, 676, L33

Slane, P., Helfand, D. J., van der Swaluw, E., \& Murray, S. S. 2004, ApJ, 616,403

Slane, P., Hughes, J. P., Temim, T., et al. 2012, ApJ, 749, 131

Smith, D. A., Guillemot, L., Camilo, F., et al. 2008, A\&A, 492, 923

Splaver, E. M., Nice, D. J., Stairs, I. H., Lommen, A. N., \& Backer, D. C. 2005, ApJ, 620, 405

Spoelstra, T. A. T., \& Hermsen, W. 1984, A\&A, 135, 135

Stappers, B. W., Bessell, M. S., \& Bailes, M. 1996, ApJL, 473, L119

Strüder, L., Briel, U., Dennerl, K., et al. 2001, A\&A, 365, L18

Sutaria, F. K., Ray, A., Reisenegger, A., et al. 2003, A\&A, 406, 245

Swanenburg, B. N., Bennett, K., Bignami, G. F., et al. 1981, ApJL, 243, L69

Takata, J., Wang, Y., \& Cheng, K. S. 2010, ApJ, 715, 1318

Tam, C., \& Roberts, M. S. E. 2003, ApJL, 598, L27

Tam, P. H. T., Kong, A. K. H., Hui, C. Y., et al. 2011, ApJ, 729, 90

Tanaka, T., Allafort, A., Ballet, J., et al. 2011, ApJL, 740, L51

Taylor, J. H., \& Weisberg, J. M. 1989, ApJ, 345, 434

The Fermi-LAT Collaboration. 2013, ApJS, submitted (arXiv:1306.6772) (1FHL)

Theureau, G., Parent, D., Cognard, I., et al. 2011, A\&A, 525, A94

Thompson, D. J. 2008, RPPh, 71, 116901

Trimble, V. 1973, PASP, 85, 579

Turner, M. J. L., Abbey, A., Arnaud, M., et al. 2001, A\&A, 365, L27

Uchiyama, Y., Funk, S., Katagiri, H., et al. 2012, ApJL, 749, L35

Venter, C., Harding, A. K., \& Guillemot, L. 2009, ApJ, 707, 800

Venter, C., Johnson, T. J., \& Harding, A. K. 2012, ApJ, 744, 34

Verbiest, J. P. W., Bailes, M., Coles, W. A., et al. 2009, MNRAS, 400, 951

Verbiest, J. P. W., Weisberg, J. M., Chael, A. A., Lee, K. J., \& Lorimer, D. R. 2012, ApJ, 755, 39

Wagner, S. J., \& Seifert, W. 2000, in ASP Conf. Ser. 202, IAU Colloq. 177: Pulsar Astronomy - 2000 and Beyond, ed. M. Kramer, N. Wex, \& R. Wielebinski (San Francisco, CA: ASP), 315

Wang, R.-B., \& Hirotani, K. 2011, ApJ, 736, 127

Wang, Y., Takata, J., \& Cheng, K. S. 2011, MNRAS, 414, 2664

Watters, K. P., \& Romani, R. W. 2011, ApJ, 727, 123

Weisskopf, M. C., Romani, R. W., Razzano, M., et al. 2011, ApJ, 743, 74

Weltevrede, P., Abdo, A. A., Ackermann, M., et al. 2010, ApJ, 708, 1426

Wu, J. H. K., Hui, C. Y., Wu, E. M. H., et al. 2013, ApJL, 765, L47

Xu, Y., Reid, M. J., Zheng, X. W., \& Menten, K. M. 2006, Sci, 311, 54

Yadigaroglu, I.-A., \& Romani, R. W. 1997, ApJ, 476, 347

Young, M. D. T., Chan, L. S., Burman, R. R., \& Blair, D. G. 2010, MNRAS, 402, 1317

Zajczyk, A., Gallant, Y. A., Slane, P., et al. 2012, A\&A, 542, A12

Zepka, A., Cordes, J. M., Wasserman, I., \& Lundgren, S. C. 1996, ApJ, 456,305

Zharikov, S. V., Shibanov, Y. A., Zyuzin, D. A., Mennickent, R. E., \& Komarova, V. N. 2008, A\&A, 492, 805 